\title{
SUPERHARMONIC NONLINEAR LATERAL VIBRATIONS OF A SEGMENTED DRIVELINE INCORPORATING A TUNED DAMPER EXCITED BY NON-CONSTANT VELOCITY JOINTS
}

\author{
A Dissertation \\ by \\ MICHAEL SCOTT BROWNE \\ Submitted to the Office of Graduate Studies of \\ Texas A\&M University \\ in partial fulfillment of the requirements for the degree of \\ DOCTOR OF PHILOSOPHY
}

May 2009

Major Subject: Mechanical Engineering 


\title{
SUPERHARMONIC NONLINEAR LATERAL VIBRATIONS OF A SEGMENTED DRIVELINE INCORPORATING A TUNED DAMPER EXCITED BY NON-CONSTANT VELOCITY JOINTS
}

\author{
A Dissertation \\ by \\ MICHAEL SCOTT BROWNE
}

\author{
Submitted to the Office of Graduate Studies of \\ Texas A\&M University \\ in partial fulfillment of the requirements for the degree of \\ DOCTOR OF PHILOSOPHY
}

\begin{abstract}
Approved by:
Chair of Committee, Alan Palazzolo

Committee Members, Make McDermott

Bryan Rasmussen

Robert Lytton

Head of Department, Dennis O'Neal
\end{abstract}

May 2009

Major Subject: Mechanical Engineering 


\begin{abstract}
Superharmonic Nonlinear Lateral Vibrations of a Segmented Driveline Incorporating a Tuned Damper Excited by Non-constant Velocity Joints. (May 2009)

Michael Scott Browne, B.S., Brigham Young University;

M.S., University of Michigan

Chair of Advisory Committee: Dr. Alan Palazzolo
\end{abstract}

Linear vibration measurement and analysis techniques have appeared to be sufficient with most vibration problems. This is partially due to the lack of proper identification of physical nonlinear dynamic responses. Therefore, as an example, a vehicle driveshaft exhibits a nonlinear super harmonic jump due to nonconstant velocity, NCV, joint excitation. Previously documented measurements or analytical predictions of vehicle driveshaft systems do not indicate nonlinear jump as a typical vibration mode. The nonlinear jump was both measured on a driveshaft test rig and simulated with a correlated model reproduced the jump. Subsequent development of the applied moments and simplified equations of motion provided the basis for nonlinear analysis. The nonlinear analyses included bifurcation, Poincare, Lyapunov Exponent, and identification of multiple solutions.

Previous analytical models of driveshafts incorporating NCV joints are typically simple lumped parameter models. Complexity of models produce significant processing costs to completing significant analysis, and therefore large DOF systems 
incorporating significant flexibility are not analyzed. Therefore, a generalized method for creating simplified equations of motion while retaining integrity of the base system was developed. This method includes modal coupling, modal modification, and modal truncation techniques applied with nonlinear constraint conditions. Correlation of resonances and simulation results to operating results were accomplished.

Previous NCV joint analyses address only the torsional degree of freedom. Limited background on lateral excitations and vibrations exist, and primarily focus on friction in the NCV joint or significant applied load. Therefore, the secondary moment was developed from the NCV joint excitation for application to the driveshaft system. This derivation provides detailed understanding of the vibration harmonic excitations due to $\mathrm{NCV}$ joints operating at misalignment angles.

The model provides a basis for completing nonlinear analysis studying the system in more detail. Bifurcation analysis identified ranges of misalignment angles and speeds that produced nonlinear responses. Lyapunov Exponent analysis identified that these ranges were chaotic in nature. In addition, these analyses isolated the nonlinear response to the addition of the ITD nonlinear stiffness.

In summary, the system and analysis show how an ITD installed to attenuate unwanted vibrations can cause other objectionable nonlinear response characteristics. 


\section{DEDICATION}

For my Family 


\section{ACKNOWLEDGEMENTS}

I would like to thank my committee chair, Dr. Palazzolo, and my committee members, Dr. McDermott, Dr. Rasmussen, and Dr. Lytton, for their guidance and support throughout the course of this research. I would like to express my gratitude for the whole committee for facilitating my unique work and school circumstances. I would like to especially show my thanks to Dr. Palazzolo for his encouragement, time, and extra effort.

I want to extend my gratitude to my current, McLaren Performance Technologies, and previous employers, Visteon/Automotive Components Holding/Ford for the use of test equipment, management support, facilitating time, and financial support.

Finally, thanks to my family for their support. Especially I want to express my gratitude to my wife for her patience and love. In addition, I want to show my appreciation for my children for their understanding of the time and effort necessary to complete this work. Also, I would like to thank my parents for the guidance and emotional support throughout the years. 


\section{NOMENCLATURE}

$\begin{array}{ll}\text { CV } & \text { Constant Velocity } \\ \text { DOE } & \text { Design of Experiments } \\ \text { FFT } & \text { Fast Fourier Transformation } \\ \text { ITD } & \text { Internal Tuned Damper } \\ \text { MDOF } & \text { Multiple Degree of Freedom } \\ \text { NCV } & \text { Non-constant Velocity } \\ \text { SDOF } & \text { Single Degree of Freedom }\end{array}$




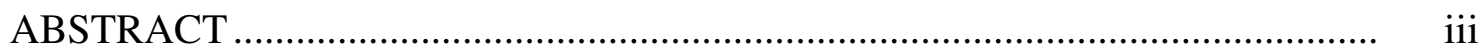

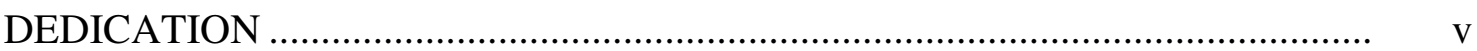

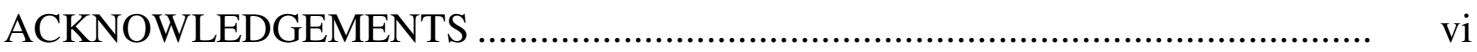

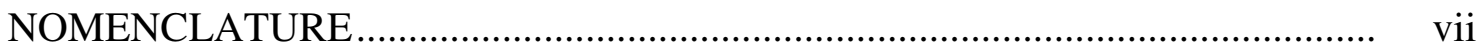

TABLE OF CONTENTS …............................................................................. viii

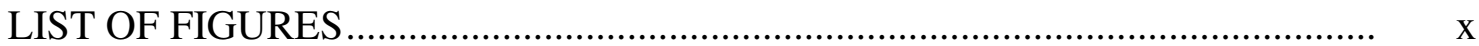

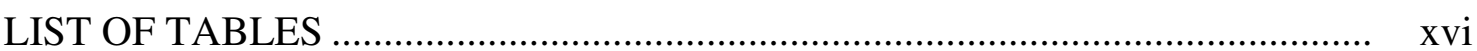

\section{CHAPTER}

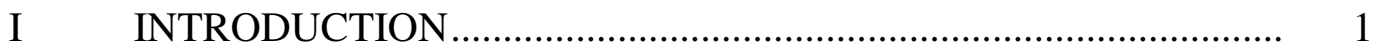

General Statement …….......................................................... 1

Background Literature............................................................... 3

Nonlinear Fundamentals ........................................................... 14

Dissertation Structure ................................................................. 20

Original Contributions of the Dissertation ....................................... 21

II DRIVELINE SYSTEM ...................................................... 24

Physical Description................................................................... 25

Test System Description.............................................................. 27

Experimental Results................................................................ 30

Summary of Driveline System and Test Results.......................... 52

III DERIVATION OF EXCITATION MOMENT …................................ 53

Non-constant Velocity Joint Characteristics ................................... 53

Derivation of the Secondary Moment ........................................... 56

Summary of Secondary Moment Characteristics........................... 64 
CHAPTER Page

IV ANALYTICAL MODEL OF DRIVELINE SYSTEM.................... 66

Derivation of Beam Model..................................................... 66

Modal Truncation .................................................................. 69

Modal Modification............................................................. 73

Modal Coupling .............................................................. $\quad 77$

Summary of Model Correlation and Simulation Results ............. 79

V $\quad$ NONLINEAR SYSTEM ANALYSIS ........................................... 93

Bifurcation and Poincare .......................................................... 94

Spectral Analysis .............................................................. 111

Lyapunov .......................................................................... 116

Summary of Nonlinear System Analysis ................................ 125

VI SUMMARY AND CONCLUSIONS ......................................... 129

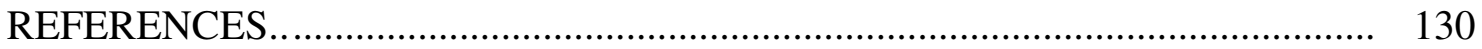

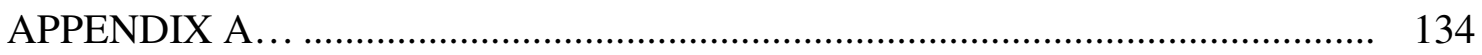

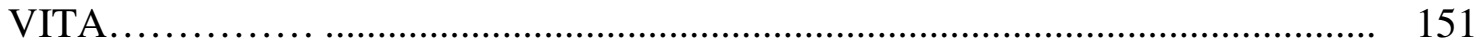




\section{LIST OF FIGURES}

Page

Fig. 1. Linear Frequency Response Near Resonance. .............................. 16

Fig. 2. Hardening (green) and Softening (red) Nonlinear Plots of Amplitude with Respect to Frequency.......................................... 17

Fig. 3. Run Up (red) and Run Down (blue) Trends of a Hardening System. 18

Fig. 4. All-wheel Drive Drivetrain Layout.............................................. 24

Fig. 5. Schematic of Driveshaft.......................................................... 26

Fig. 6. a)Entire Driveshaft Assembly, b) Constant Velocity Joint at PTU/Transaxle End of Driveshaft, c) Center Bearing Assembly with Non=Constant Velocity Joint, and d) Axle End of Driveshaft Assembly

Fig. 7. Schematic of Test Stand......................................................... 28

Fig. 8. Test Rig Instrumentation on Center Bearing.................................. 29

Fig. 9. Lateral Acceleration Measured at the Active Side of the Center Bearing during a) Run Up, b) Run Down, and c) Run Up with no Driveshaft.

Fig. 10. Run Up Order Spectrum of Lateral Vibration at Active Side of the Center Bearing.

Fig. 11. Measured Acceleration at Center Bearing Active Side of Support Mount at 0 (red) and 4 Degrees (blue) of Misalignment.....

Fig. 12. Order Spectrum for Run Up for a) 0 Degrees of Misalignment and b) 4 Degrees of Misalignment.

Fig. 13. Order Comparison for a) 2nd Order, b) 4th Order, c) 6th Order, and d) 8th Order with Misalignment Angles of 0 (blue), 2(red), and 4 Degrees (magenta)

Fig. 14. 1st Order Response at 4 Degrees of Misalignment Due to Imbalance with Added 0 (blue), 4 (red), 13 (magenta), and 25 Grams (yellow).

Fig. 15. Comparison of Order Spectrum Response with 4 Degrees of Misalignment for a) 0 and b) 25 Grams. 
Fig. 16. Order Response at Multiple Imbalance Levels a) $2^{\text {nd }}$ Order, b) $4^{\text {th }}$ Order, c) $6^{\text {th }}$ Order, and d) $8^{\text {th }}$ Order at Imbalance of Added 0(blue), 4 (red), 13 (magenta), and 25 Grams (yellow).

Fig. 17. Repeatability of Test Measurements at 0 Grams and 0 Degrees for a) 1 st Order, b) 2nd Order, and 25 Grams and 4 Degree Misalignment for c) 4th Order, and d) 8th Order.

Fig. 18. 6th Order Repeatability Response at 0 Grams of Added Imbalance with a) 0 Degrees and b) 4 Degrees Misalignment........................ 44

Fig. 19. Center Bearing Bounce Mode at 16.5 Hz.

Fig. 20. Driveshaft Modes: a) Front Shaft Bending/ ITD Lower at $95 \mathrm{~Hz}$ and b) Front Driveshaft Bending/ ITD Upper at $146 \mathrm{~Hz}$.

Fig. 21. Driveshaft Modes: a) Rear Shaft Bending at $162 \mathrm{~Hz}$ and b) Front Driveshaft Second Bending at $385 \mathrm{~Hz}$.

Fig. 22.

Stiffness Measurements on ITD 49

\section{Fig. 23.}

Schematic of ITD in Driveshaft. 50

Moment Stiffness Measurement of Driveshaft Center Bearing.

Fig. 25. Depictions of Shaft System Relative Rotations due to Misalignment.

Fig. 26.

Universal Joint Coordinate System.

Fig. 28. Comparison of Full Moment and Fourier Series Approximation.....

Fig. 29. Comparison of Full Moment and Fourier Series Approximation of Secondary Moment.

Fig. 30.

Beam Element Coordinate System Definition.

Fig. 32. Simulation Run Up Vibrations at a) ITD and b) Driveshaft at Shaft to ITD Connection Node.

Fig. 33. Angle Response at Center Universal Joint for a) Front Shaft Angle, b) Rear Shaft Angle, and c) the Total Relative Angle Between Shafts.

Fig. 34. $\quad$ Measured Center Bearing Response Top X-dir. ............................. 83

Fig. 35. Measured Center Bearing Top Z-dir. 
Fig. 36. Contour Plot of Simulated Vibrations of the Rear Shaft Joint Angle.

Fig. 37. ITD Response Comparison of Full Force Excitation (blue) and Fourier 4th Order Excitation (green).

Fig. 38. Driveshaft Response Comparison of Full Force Excitation (blue) and Fourier 4th Order Excitation (green).

Fig. 39. Fourier Series with Only $4^{\text {th }}$ Order Contribution Contour Plot. 88

Fig. 40. Linear Simulation Results of a) ITD and b) Driveshaft Response at ITD Location.

Fig. 41. Plot of the Simulated Response with Low Damping During Run Up (blue) and Run Down (green).

Fig. 42. Sample Response Oscillations with Identification of Points of First Returns.

Fig. 43. Phase Phase-Plane Plot of Sample Oscillations.

Fig. 44. Bifurcation Diagram with 4 Degree Misalignment Across 4x Input Frequencies of $2-250 \mathrm{~Hz}$ with $4^{\text {th }}$ Order Excitation Only.

Fig. 45. Bifurcation Diagram with 4 Degree Misalignment with Finer Frequency Resolution for $80-150 \mathrm{~Hz}$ with $4^{\text {th }}$ Order Excitation Only.

Fig. 46. Bifurcation Diagram with 4 Degree Misalignment Across 4x Input Frequencies of 2-250 Hz with Full Force Excitation.

Fig. 47. Bifurcation Diagram with 4 Degree Misalignment with Finer Frequency Resolution for 80-215 Hz with Full Force Excitation.....

100

Fig. 48. Bifurcation Diagram with 4 Degree Misalignment with Finer Frequency Resolution for 124-140 Hz with Full Force Excitation...

Fig. 49. Bifurcation Diagram Across Misalignment Angles of 0-23 Degrees with $4^{\text {th }}$ Order Excitation Only at $100 \mathrm{~Hz}$.

Fig. 50. Bifurcation Diagram Across Misalignment Angles of 0-23 Degrees with $4^{\text {th }}$ Order Excitation Only at $135 \mathrm{~Hz}$.

Fig. 51. Bifurcation Diagram Across Misalignment Angles of 10-15 Degrees with $4^{\text {th }}$ Order Excitation Only at $135 \mathrm{~Hz}$. 104

Fig. 52. Bifurcation Diagram Across Misalignment Angles of 11.74-11.84 Degrees with $4^{\text {th }}$ Order Excitation Only at $135 \mathrm{~Hz}$. 
Fig. 53. Plot of First Returns on a) Bifurcation Diagram and b) Poincare Plot for 11.8325 Degrees of Misalignment with $4^{\text {th }}$ Order Excitation Only at $135 \mathrm{~Hz}$.....

Fig. 54. Time Response of Front Driveshaft at ITD Location with 11.8325 Degrees of Misalignment Simulated at $135 \mathrm{~Hz}$.

Fig. 55. Plot of First Returns on a) Bifurcation Diagram and b) Poincare Plot for 14.5 Degrees of Misalignment with $4^{\text {th }}$ Order Excitation Only at $135 \mathrm{~Hz}$.

Fig. 56. Poincare Plot for 14.5 Degrees of Misalignment with $4^{\text {th }}$ Order Excitation Only at $135 \mathrm{~Hz}$ with 320,256 cycles.

Fig. 57. Poincare Plot for 14.5 Degrees of Misalignment Full Moment at $135 \mathrm{~Hz}$ with 320,256 cycles.

Fig. 58. Time Response of Front Driveshaft at ITD Location with 14.5 Degrees of Misalignment Simulated at $135 \mathrm{~Hz}$.

Fig. 59. Contour Plot of Frequency Spectrums at each Misalignment Angle from 0-20 Degrees at $135 \mathrm{~Hz}, 4 x$ Excitation.

Fig. 60. Contour Plot of Frequency Spectrums to $200 \mathrm{~Hz}$ at each Misalignment Angle from 10-15 Degrees at $135 \mathrm{~Hz} 4 \mathrm{x}$ Excitation..

Fig. 61. Frequency Spectrum to $200 \mathrm{~Hz}$ at 14.5 Degrees of Misalignment from $1000 \mathrm{~Hz}$ Sampling Rate and Blocksize of 1024 Samples.

Fig. 62. Frequency Spectrum to $200 \mathrm{~Hz}$ at 14.5 Degrees of Misalignment from $1000 \mathrm{~Hz}$ Sampling Rate and Blocksize of 4096 Samples on a Log Scale.

Fig. 63. Frequency Spectrum of Simulation Results with 1 Degree Misalignment.

Fig. 64. Time Response of the Calculation of the Maximum (blue) and Minimum (green) Lyapunov Exponents at 14.5 Degrees of Misalignment Excited at $135 \mathrm{~Hz}$.

Fig. 65. Time Response of the Calculation of the Maximum (blue) and Minimum (green) Lyapunov Exponents at 1 Degree of Misalignment Excited at $135 \mathrm{~Hz}$.

Fig. 66. Converged Maximum (green) and Minimum (blue) Lyapunov Exponents for Misalignment Angles of 1 to 20 Excited at $135 \mathrm{~Hz} .$. 
Fig. 67. Time Response of Front Driveshaft at ITD Connection Location for Misalignment Angles of: a) 1, b) 10, c) 14.3, d) 16.7, and e)18.5 Degrees.

Fig. 68. Lyapunov Exponents for Misalignment Angles 0.5 - 20 Degrees without ITD Nonlinear Stiffness.

Fig. 69. Bifurcation of System without ITD Nonlinear Stiffness.

Fig. 70. Overlapping Plots of a Run Up (blue) and Run Down (green) Simulation with Reduced Damping.

Fig. 71. Steady State $4^{\text {th }}$ Order Force Excitation Response Simulation from Run Up (blue) and Run Down (green) Initial Conditions ................

Fig. 72. Zoomed In on Time Period Steady State $4^{\text {th }}$ Order Force Excitation Response Simulation from Run Up (blue) and Run Down (green) Initial Conditions.

Fig. 73: Calculated Duffing Response with $\mathrm{k}=0.3, \omega=1.2$, Gamma $=0.5$ of a) Lyapunov Maximum (blue) and Minimum (green) Exponents and b) Time Response.

Fig. 74. Calculated Duffing Response with $\mathrm{k}=0.3, \omega=1.2$, Gamma=0.2 of a) Lyapunov Maximum (Blue) and Minimum (green) Exponents and b) Time Response.

Fig. 75. Calculated Duffing Response with $\mathrm{k}=0.3, \omega=1.2$, Gamma=0.28 of a) Lyapunov Maximum (blue) and Minimum (green) Exponents and b) Time Response.

Fig. 76. Calculated Duffing Response with $\mathrm{k}=0.3, \omega=1.2$, Gamma=0.29 of a) Lyapunov Maximum (blue) and Minimum (green) Exponents and b) Time Response.

Fig. 77. Calculated Duffing Response with $\mathrm{k}=0.3, \omega=1.2$, Gamma=0.37 of a) Lyapunov Maximum (blue) and Minimum (green) Exponents and b) Time Response.

Fig. 78. Calculated Duffing Response with $\mathrm{k}=0.3, \omega=1.2$, Gamma=0.65 of a) Lyapunov Maximum (blue) and Minimum (green) Exponents and b) Time Response.

Fig. 79. Calculated Duffing Response with $\mathrm{k}=0.3, \omega=1.2$, Gamma=0.73 Pre-simulation Start of 1.5,0 of a) Lyapunov Maximum (blue) and Minimum (green) Exponents and b) Time Response.

Fig. 80. Calculated Duffing Response with $\mathrm{k}=0.3, \omega=1.2$, Gamma=0.73 Pre-simulation Start of 0,0 of a) Lyapunov Maximum (blue) and Minimum (green) Exponents and b) Time Response 
Fig. 81. Lyapunov Maximum (blue) and Minimum (red) Exponents for the Lorenz Equation with $\sigma=16, R=45.92$, and $b=4$ Using Natural Log.

Fig. 82. Maximum (blue) and Minimum (red) Exponents for the Lorenz Equation with Sigma=16, T=45.92, and Beta=4 Using Log Base 2. 149

Fig. 83. Maximum (blue) and Minimum (red) Exponents for the Rossler Chaos with $\mathrm{a}=0.15, \mathrm{~b}=.20$, and $\mathrm{c}=10.0$ Using Log Base 2.............. 


\section{LIST OF TABLES}

Page

Table $1 \quad$ Experimental Identification of Driveshaft Natural Frequencies ...... 46

Table $2 \quad$ Front Driveshaft Natural Frequencies ...................................... 70

Table $3 \quad$ Rear Driveshaft Natural Frequencies ........................................ 70

Table $4 \quad$ Comparison of Analytical and Experimental Natural Frequencies... 80 


\section{CHAPTER I}

\section{INTRODUCTION}

\section{General Statement}

Typical industrial vibration problem solving includes utilization of linear vibration measurement and analysis techniques. These techniques have appeared to be sufficient with most vibration problem solving requirements. This is due to the lack of high quality measurements of the nonlinear dynamic response of actual engineering systems. This lack of high quality response data of nonlinear dynamic systems prevents the correlation of analytical models to physical test response.

Therefore, as an example, a vehicle driveshaft exhibits a nonlinear super harmonic jump that has not been previously documented in measurement or analytical prediction. Therefore, it is intended that this practical example will contribute effective measurement methods, theoretical analysis and simulation models for nonlinear dynamic response analysis of these systems.

Traversing distances is necessary to transfer mechanical power from the power source to the required location for the utilization of the energy. Mechanical transfer of power for machinery typically requires rotating shafts of various types. These shafts may comprise of various elements including gears that can change the rotational axis as needed. Gears, hypoid and spiral bevel, help to correct for large changes in rotational

This dissertation follows the style of Journal of Sound and Vibration. 
axes, but are not practical to compensate for small misalignments. Various installation tolerances of the input power source and the output mechanical operation cause these small misalignments. High level of geometric installation accuracy can align the input and output properly. Due to cost and time, this is not practical for most installations of shafted systems. Therefore, installation of various couplings and joints in a shafted mechanical system is to compensate for these small misalignments.

An example of a system that requires a shaft(s) to transfer mechanical energy is a typical passenger vehicle. The engine is the mechanical power source. The vehicle then requires gearing and shafts to transfer the mechanical energy to the wheels. The torque transferred to the wheels reacts with the ground producing a force that propels the vehicle. The vehicle's drivetrain is this shafting system. A typical drivetrain for a rear wheel drive vehicle includes a transmission, driveshaft, axle differential, and axle shafts.

The driveshaft typically provides allowance for misalignment. Misalignment in a vehicle is due to many factors including manufacturing tolerances, packaging constraints, suspension travel, and engine dynamic movement (pitch and roll). Nonconstant velocity (universal joint or Hooke's joint), constant velocity (CV joint), and flex couplings have been used in vehicle driveshaft applications for these reasons. The most common coupling used in driveshafts is the non-constant velocity joint. This is due to the non-constant velocity's capabilities to transfer significant torque at relatively large amounts of misalignment $\left(5-10^{\circ}\right)$. 


\section{Background Literature}

There exists a significant amount of knowledge of basic kinematics of nonconstant velocity (NCV) joints. The kinematic relationship produces a difference of rotational position and velocity of the output relative to the input. Also due to the change of direction of applied moment to output moment, additional moments are produced as outlined by Porat[1]. Current analyses of NCV joints include application to mechanisms in a six bar configuration[2] and nonlinear response in positioning (but nonrotating) equipment[3]. These static or pseudo static systems involve complex analysis of the joints with respect to kinematics.

More interestingly, the physical kinematic relationship produces a second order, or two times per revolution, periodic angular speed fluctuation of the output shaft in spite of the input driven at a constant velocity through the NCV joint $[4,5]$. The difference between the input and output angular speeds has been defined as the Cardin Error[6]. This was also outlined for general rotor dynamic applications by Porat[1]. Therefore, the nomenclature, non-constant velocity joint is applicable. The kinematic relationship explains the increase in amplitude of the periodic fluctuations of velocity as the relative u-joint angle is increased.

Research and analysis of systems incorporating NCV joints have been completed. These systems include various system configurations including: single joint systems, two joint systems with a shaft between the joints, and three joint systems with shafts between each joint. In addition most of the research is in the Torsional vibration of these systems, with a lesser amount of research into the lateral vibrations of these 
systems. The following sections present the background research into these systems grouped by the vibration response and then by the physical configuration of shaft and joints. Subsequent to these sections, sections addressing the type of analytical models, analytical methods and empirical testing and analysis performed by previous researches will be summarized.

\section{Torsional Vibration Response}

Single Joint Systems

There has been significant research into the torsional effects of a single NCV joint system. Porter initially analyzed the torsional linearized equations of motion to determine critical speed ranges of large amplitude oscillations due to a NCV joint[7]. Porter concluded that critical speed ranges exist for input speeds equal to the natural frequency or integer multiples of the natural frequency. Porter concluded that linearized equations using Floquet theory produce only the stability regions and not magnitudes of the amplitudes of vibrations.

Subsequently, Porter initiated nonlinear analytical analysis of the torsional response of NCV joint system including a SDOF and two degree of freedom system (2DOF)[8]. Response of SDOF analysis provided analysis into limit cycle oscillations due to the NCV joint. Porter predicted again that violent oscillations would occur at each of the torsional natural frequencies of the flexible shaft NCV system. Porter predicted that multiple degree of freedom systems with multiple u-joints would only superimpose instability regions based on the systems torsional natural frequencies. In 
addition, sub harmonics were predicted as possible, but harmonics were deduced to be of lower amplitude, and thus less consequential[8].

More recently, Chang identified torsional instabilities by using the method of strained parameters to determine instability regions of Porter's single NCV joint system[9]. Chang concluded that the ratio of the input to the output shaft stiffness is a factor in determining the instability region. Asokanthan also analyzed the 2DOF system, but utilized initially the method of averaging and later Lyapunov exponents to determine instabilities $[10,11]$. Asokanthan further analyzed the 2DOF system with additional application of the method of averaging, numerical simulations, and bifurcation analysis[12]. These analytical analyses addressed the torsional response of the same model incorporating a single NCV joint system with fixed bearings.

Mazzei introduced soft support boundary conditions on the output shaft with torsional inertia $[13,14]$. This model included flexible shafts but without significant inertia. Mazzei concluded that parametric instability for this system in the operational speeds of a truck application is due to the rigid body and shaft flexible modes of the system. Mazzei also addressed flutter response. The flutter instabilities occur at shaft speeds that are significantly higher than typical operation speeds. Therefore, the instabilities of these systems are parametric instabilities excited by the bending moment produced by the NCV joints.

Desmidt furthered research into the soft support boundary conditions on the output shaft including both lateral and torsional response of the output shaft[6]. Desmidt concluded that the response of the system involves coupled torsional and lateral 
responses. Stability was determined utilizing Floquet theory and additional perturbation analysis. Desmidt identified the need for additional damping of the output shaft to maintain stability through the combination zones.

Dimentberg continued with the $2 \mathrm{DOF}$ system with a NCV joint that produced random transverse vibrations that was excited by support base excitation $[15,16]$. Dimentberg utilized stochastic averaging and other statistical tools to analyze the instabilities. These vibrations are an exception to the typical transverse vibrations caused by imbalance and misalignment, which are first order, or once per revolution excitations.

\section{One Shaft, Two Joint Systems}

Zahradka analyzed the torsional response of a two NCV joint system using Van der Pol's method of slowly varying coefficients[17]. The system includes two NCV joints separated by an intermediate shaft, with the outer shafts rigidly supported. This system is similar to a short wheelbase vehicle with a one-piece driveshaft system. Zahradka predicted torsional critical speed ranges at one-half times and one times the first natural frequency of the system. Zahradka also commented on experimental results with other sub-harmonic torsional critical speed ranges.

Two Shafts, Three Joint Systems

Desmidt continued with more complexity by introducing a segmented driveline for analysis[18]. The driveline incorporated three NCV joints, and analyzed the 
torsional response. This system is closer to a vehicle long wheelbase, but had a fixed support on the center bearing location. This driveline included only fixed bearing supports with external dampers located at the two shafts mid-point. These dampers allow for supercritical operation. Desmidt applied Floquet theory, particularly the application of the eigenvalues of the Floquet Transition Matrix for analysis. Desmidt continued his analysis to include active magnetic bearings to replace the external dampers to control the shafts lateral vibrations[19]. This study included the closed loop feedback controls of the magnetic bearings.

\section{Lateral Vibration Response}

Single Joint Systems

There exists limited initial research into lateral vibrations caused by $\mathrm{u}$-joint. This is in spite of identification of a moment normal to a shaft caused by the NCV joint by Porat[1]. Ota completed static measurement and analysis of the this moment, sometimes referred as the secondary moment[20]. Friction of the NCV joint pins do not generate this moment as the analysis assumed no friction and experimental setup minimized the frictional influence. Ota predicted violent lateral vibrations when an even integer multiple of shaft speed corresponded with the natural frequency.

Ota continued with analysis and experimental setup, concluding that the secondary moment will cause lateral vibrations of even multiples of the driveshaft angular speed[21]. Ota also concluded that violent vibrations would occur when these multiples align with natural frequencies of the drive shaft system. 
The response of both misalignment across the NCV joint and imbalance were considered in analysis and testing by $\mathrm{Xu}$ [22]. Xu concluded that either the unbalance or the misalignment excitation would be amplified when the excitation frequencies or the even multiples of shaft speed are near a system natural frequency.

Plaut identified rotordynamic combination and difference instabilities involving parametric excitation of both torsional and flexural natural frequencies of a simple rotor[23]. Iwatsubo discovered both parametric and self excited lateral vibrations utilizing the Routh-Hurwitz criterion of the 2DOF system with the soft supported output shaft[24]. Iwatsubo also concluded that asymmetric support stiffness improves stability while the gyroscopic moment destabilizes an asymmetric stiffness matrix.

Kato investigated the lateral response due to the friction between the NCV pin and the shaft yoke in a NCV joint setup[25]. Kato presented analysis and experimental results with a fixed input and pinned output shaft. Kato concluded that viscous frictional excitation of a NCV joint will increase with increased angle for all even harmonics of the shaft speed, while Coulomb will not increase with speed and predominantly increase the fourth and eighth harmonics the shaft speed. Desmidt analyzed some coupled-lateral and torsion response of the softly supported output shaft model[6]. Desmidt concluded that the periodic inertia coupling matrices couple the torsion and lateral response that produce sum type combination response frequencies.

One Shaft, Two Joint Systems

Initial analysis into a shaft system that simulates one type of an automotive drive- 
line system, one-shaft driveshaft system, was completed by Rosenberg[26]. Rosenberg analyzed the effects of NCV joint excitation on the critical speed of a driveshaft, the speed that a driveshaft will become unstable due to the first bending mode. Rosenberg predicted that low torque transmission of this drivetrain would produce mild instabilities at odd submultiples of the critical speed (first bending mode of the driveshaft). In addition, including torque would excite even submultiples of the critical speed, which decrease in response with increased torque loading.

Sheu furthered the analysis of vibratory response of the one shaft and two NCV joint system[27]. Sheu's model included soft supports of the entire shaft system. Sheu calculated the amplitude of the steady state response of this system including the amplitude of the harmonics separately using Finite Element Analysis (FEA). Sheu concluded that odd harmonics are due to shaft imbalance, and the even harmonics are due to the NCV joint friction.

Two Shafts, Three Joint Systems

Earles presented primarily experimental data discussing limit cycles with three NCV joint systems, including a four shaft system that most accurately simulates a twoshaft automotive driveline system[28]. The four-shaft system involves soft support of the bearing supporting the center $\mathrm{NCV}$ joint. Earles predicted jump type phenomena, or system oscillation hysteresis, in run-up and run-down situations, but lacked sufficient data[28]. In addition, the data presented was data from extremely large NCV joint angles between the input and output shafts. Earles reported that the largest maximal 
lateral excitation occurs at one half and one times the lateral natural frequency. Further, Earles predicts unstable vibration above the lateral natural frequency, attributed to friction in the joint bearings.

Saigo continued work into frictional influence on universal joints investigating a cross groove constant velocity (CGCV) joint[29]. Saigo predicted and tested the Coulomb frictional contribution to instabilities of a shaft with one CGCV joint and two NCV joints. This system is similar to a typical automotive driveshaft. The results show a significant jump-up in amplitude as rotational speed is increased.

\section{Analytical Model Definition}

Fixed supports constrain the input, the output, or both shafts of most of the presented studies, and therefore the NCV joint has no translational vibration components. This includes the studies that softly supported the output shafts by Mazzei and Desmidt $[6,13,14]$. Sheu's applied soft supports of the input and output motor assembly, thus similar to a once piece automotive application allowing for small lateral vibrations at the u-joint[27]. A typical vehicle two-piece driveline has soft support at the center NCV location for a segmented driveline. This soft support is typically composed of elastomeric material retained by a stamped steel bracket. These designs typically incorporate voids or other geometric designs that generate significantly nonlinear forcedeflection curves. Thereby, large lateral vibrations are possible at the center NCV joint that previously has not been modeled and analyzed. In addition, all models were mocked up and not representative of an actual automotive driveshaft system. 
In addition, the creation of a useful nonlinear dynamical model is difficult. Typical nonlinear models are small, 1-4 DOF due to the complexity of simulation and analysis. Examples are Duffing, Rossler-chaos, Lorenz, and others. A complex system is more difficult to analyze. Many attempts of simplifying equations of motion for nonlinear analysis have been completed. This includes using nonlinear normal modes. Methods for constructing nonlinear normal modes include using Galerkin [23, 30, 31] and Proper Orthogonal Decomposition (POD) [32-34] methods. Pak introduced a generalized form of coupling nonlinear normal modes[35]. Difficultly arrives in nontraditional forms of nonlinearity, and is therefore limited in application. Therefore, a more generalized approach to adding nonlinear components to a reduced degree of freedom system similar to a driveshaft system is desired.

\section{Analytical Analysis Methods}

Primarily, previous research work is primarily to determine stability, and more important, identify instability regions. Multiple methods applied to NCV joint analysis isolated these stability/instability regions. These methods include typical eigenvalue analysis for linearized equations and then followed by typical rotordynamic analysis as summarized by Khader[36] of utilizing of Floquet theory and techniques[6, 7, 9, 18, 19, $36]$ and the Mathieu equation[7, 26].

In addition, nonlinear methods including the method of averaging $[9,11,12,15]$, Lyapunov [10, 12], Kryloff and Bogoliuboff's method[8], stochastic averaging[15], Van der Pol's method of slowly varying coefficients[17], the Routh-Hurwitz criterion[24], 
energy balance and Fourier Expansion[29] have also been utilized analysis and determine stability and system response. In particular, the Lyapunov analysis was used to determine the stability of the driveshaft system being parametrically exited due to the NCV joint excitation. Outside of driveshaft systems, Lyapunov Exponent analysis for determination of chaotic responses have been completed on rotor to stator rub[37], and cracked rotor vibration problems[38].

Besides stability, time or RPM response amplitudes can also be calculated to simulate numerically the system. Methods including Finite Element Analysis (FEA)[27], component mode synthesis[22], and Runge-Kutta-Gill methods[29] were used to simulate and calculate particularly the amplitude response of the system harmonics.

\section{Empirical Results and Analysis}

Background research produced a lack of significant experimental data for analysis results and comparison. Many researchers alluded to experimental results without publication of any experimental data.

Presented experimental results include Kato's experiment with friction between the NCV joint pin and yoke[25]. Kato presented amplitude peaks due to harmonics passing through a resonance, showing typical linear response from harmonic excitation. This experiment is not realistic of a typical automotive application of a NCV joint that utilize rollers in the NCV joints between the pins and rollers, thus eliminating the 
frictional moment studied, more similar to that analyzed and measured by Ota [20, 21]. These experiments do not represent typical automotive driveline systems.

Earles also presented limited experimental data to extremely large u-joint angles and attributed instabilities to NCV joint friction[28]. Earles comments on jump type phenomena without any supporting data and analysis to back up the claim. The data presented only reports amplitude peaks at system resonances due to second harmonic of shaft speed. Therefore, even though the system is most representative of a two-shaft driveshaft automotive system, the experimental results do not provide enough information to support the claims.

$\mathrm{Xu}$ completed experiments with flexible couplings (no NCV) to support his previous analytical analysis of misalignment of shaft systems[39]. Xu showed experimentally the effects of two times running speed vibrations due to misalignment as well as amplification and beating with known natural frequencies.

Research into nonlinear superharmonic jump is lacking in analyzing the driveshaft system. Analysis of controlling Superharmonic resonances for rotor magnetic bearing system, and general control of a SDOF system have been presented by Ji [40, 41]. Additional numerical calculation involving elastomeric hysteresis predicting possible nonlinear jumps have been presented by Lacarbonara[42].

\section{Conclusions from Previous Research}

The presented experimental data does not conclusively explain or show super harmonic nonlinear dynamic lateral vibration response including jump type phenomena 
due to NCV joint excitations in a driveshaft system. Saigo reported a jump of increased magnitude due to a CGCV joint excitation caused by Coulomb friction as the rotational speed is increased[29]. Other than Saigo, the presented experimental results lack key characteristics where the system is mocked up, and therefore not applicable to a typical automotive driveline. In addition, typical automotive driveshafts have elastomeric supports that include voids or other geometric factors that create nonlinear force deflection response. Finally, the presented empirical data only involved time or steady state FFT signal processing with limited correlation of system response and analytical model used.

\section{Nonlinear Fundamentals}

The fundamental characteristics of dynamic systems and the key differences from linear response are vital for both the identification and understanding of these systems. These fundamental characteristics include a dependence on initial conditions, possibility of multiple solutions with the same input to the system, a response with multiple response frequencies with only a single frequency input, and the natural frequencies changing with respect to amplitude. The last characteristic of changing natural frequencies creates amplitude level jumps during excitation frequency run-up and run down events.

A linear physical vibration system comprising of a spring, mass, and damper elements generates a response of a harmonic solution, same frequency response as the 
excitation frequency, with an amplitude dependant on the excitation frequency. Such a system has the form of

$M \ddot{x}+C \dot{x}+K x=f * \sin (\omega t)$

This system can only have harmonic responses due to the interdependence of the displacement, velocity, and acceleration terms defined by differentiating the displacement as

$$
\begin{aligned}
& x=A^{*} \sin (\omega t) \\
& \dot{x}=A \omega^{*} \cos (\omega t) \\
& \ddot{x}=-A \omega^{2} \sin (\omega t) .
\end{aligned}
$$

Therefore, the response across all frequency range can easily be summarized in a Bode plot with a curve representing the amplitude response. This curve is pictured in Fig. 1. 


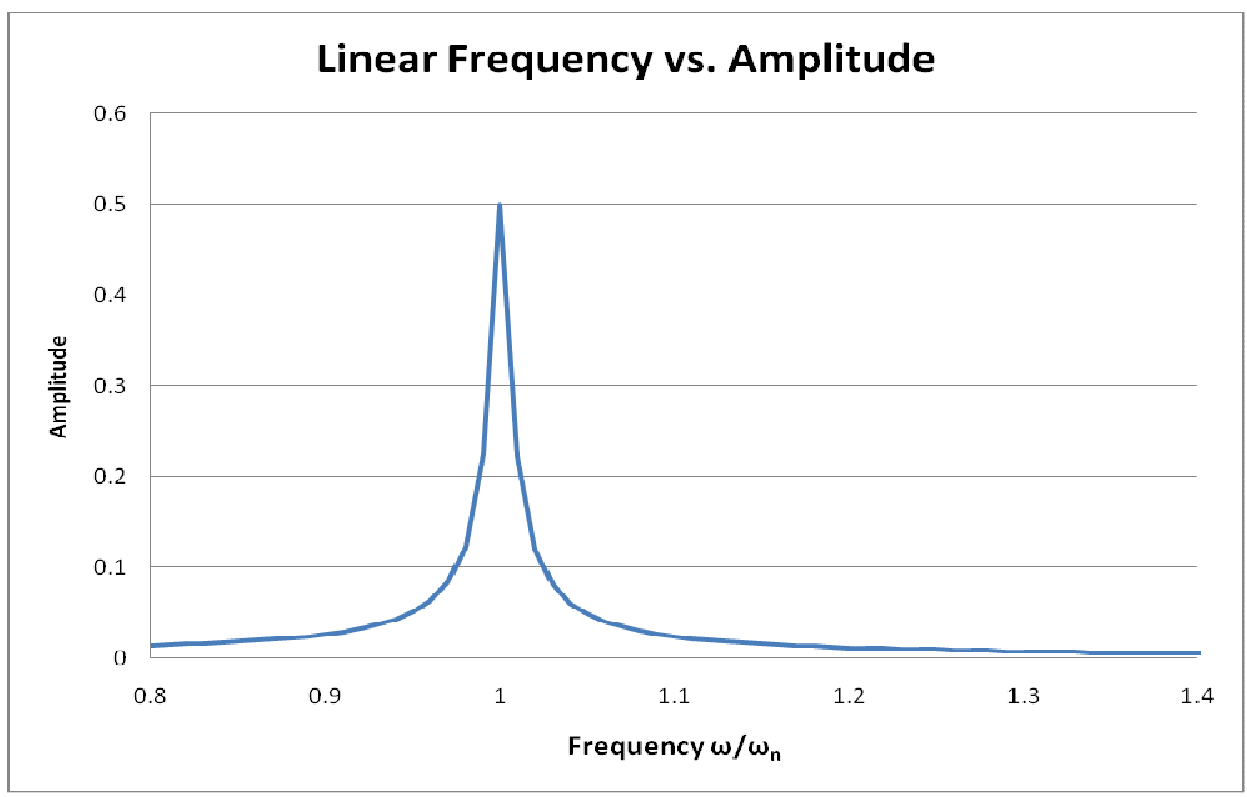

Fig. 1. Linear Frequency Response Near Resonance.

The nonlinear system has extra terms in the equation that complicate the response of the system. An example of such a system is

$$
M \ddot{x}+C \dot{x}+K x+K_{c u b e} x^{3}=f * \sin (\omega t)
$$

This system has an extra term with a cubic stiffness term. This cubic stiffness at a significant level can significantly alter the response to an excitation. The complexity comes with the cubic term. If the same displacement result is assumed, the cubic term becomes

$$
x^{3}=(A \sin (\omega t))^{3}=-\left(\frac{1}{4}\right) \mathrm{A}^{3} \sin (3 \omega t)+\left(\frac{3}{4}\right) \mathrm{A}^{3} \sin (\omega t)
$$


after simplifying and applying trigonometric identities. Therefore, it is apparent how nonlinear systems can have responses with frequencies other than the forced input frequency. Depending on the specific nonlinear system, this multiple frequency phenomena subsequently can produce sub harmonics, and other frequency content. As more frequencies are present, the system will eventually proceed to chaotic vibration behavior where the vibration is no longer converging to a stable solution, but is diverging into a solution that is constantly changing.

Depending on the sign of the cubic stiffness coefficient, the Bode plot changes. This can be seen in Fig. 2 for a softening $(K<0$ and hardening $K>0)$ spring stiffnesses.

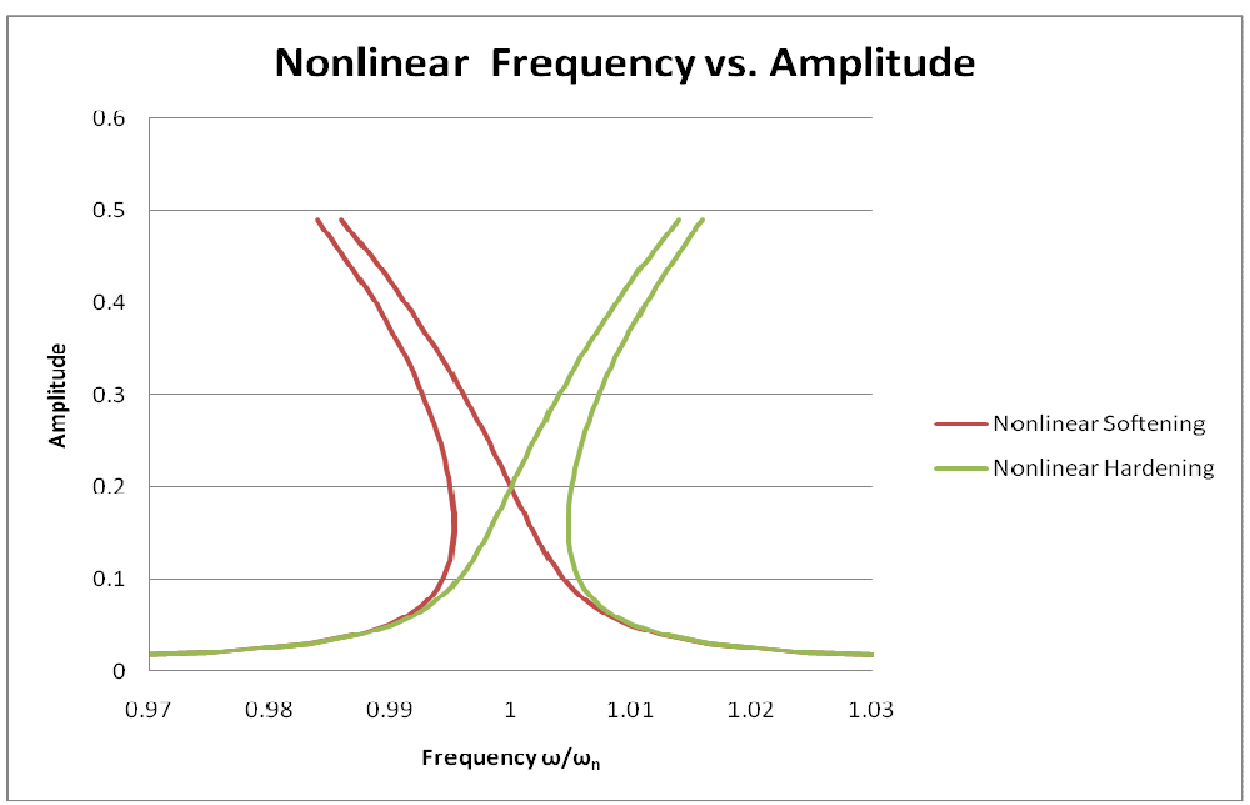

Fig. 2. Hardening (green) and Softening (red) Nonlinear Plots of Amplitude with Respect to Frequency. 
The Bode plot now shows range of Frequencies where multiple amplitude solutions are possible. For any nonlinear system, there as many possible solutions as is the order of the nonlinearity. Therefore for the cubic term presented, there are up to 3 solutions for a given frequency input. The solutions can be each individually simulated by stating with the appropriate initial conditions at the frequency to guide the response to the particular solution. The initial conditions will tend to one of the solutions, and have been termed as attractors.

During a ramp-up and ramp-down condition, the response follows the corresponding amplitude on the Bode plot. For a spring hardening system, this results in a jump down of amplitude at higher than the linear natural frequency and a jump up at a frequency lower than the linear natural frequency. These jumps are depicted in Fig. 3.

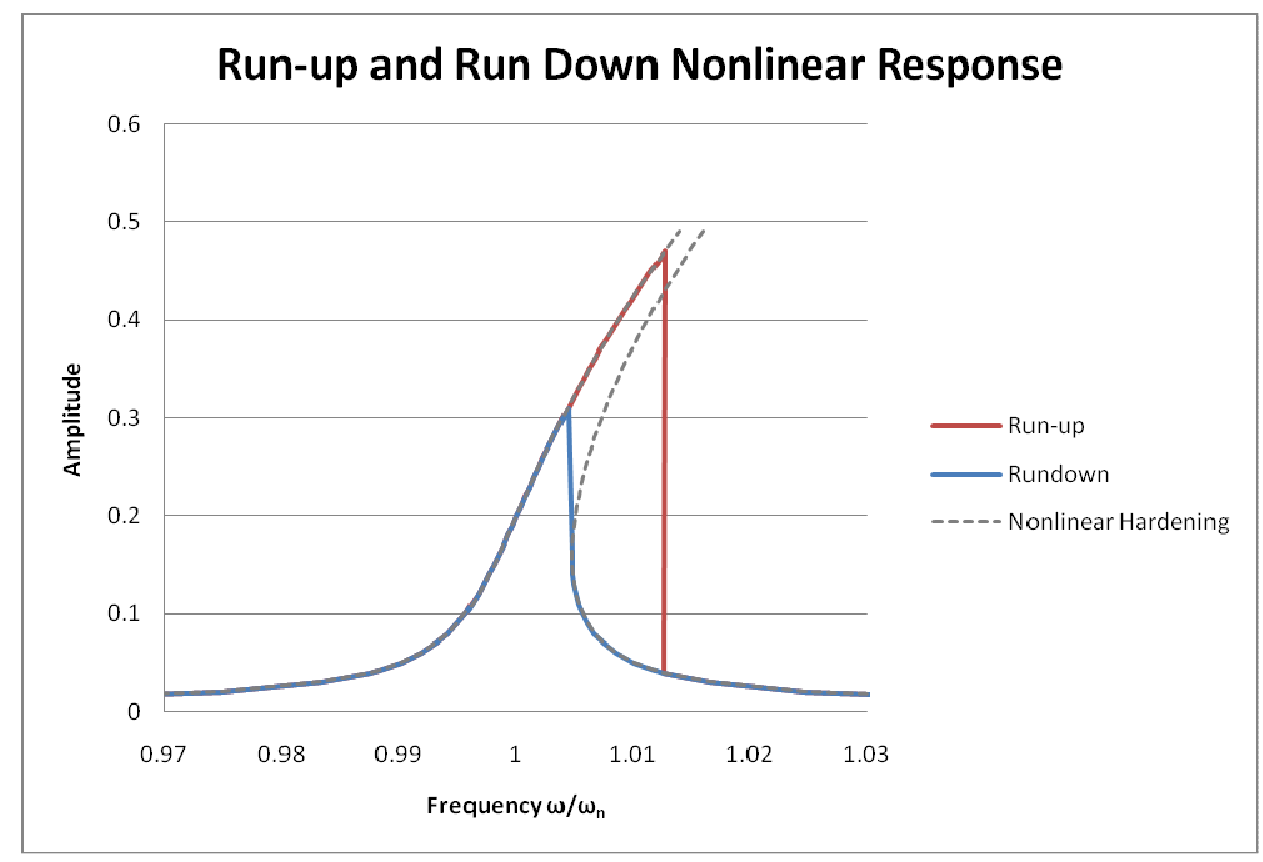

Fig. 3. Run Up (red) and Run Down (blue) Trends of a Hardening System. 
The response shows a different level of amplitude jump for the run-up and run down conditions. This is an important characteristic for identifying nonlinear jump behavior, and separating it from other jump influences such as parametric excitation.

The natural frequency is defined from a linear system of equations. The natural frequency of a nonlinear system is dependent on the linear interpolation of the system of equations. The system can be interpolated at ranges of different amplitudes of vibration. At these amplitudes, the stiffness is higher or lower depending on the cubic term being spring hardening or spring softening. This is important as excitation and response of the systems can provide different natural frequencies depending on the response amplitude. This can be most sensitive to physical modal testing of a system. Typical model tests assume linearity, and only calculate the natural frequency based on small vibration amplitudes. Therefore, physical test results identifying natural frequencies in a nonlinear system need to consider amplitude levels to base confidence of the results.

Therefore, a nonlinear system can produce multiple response solutions and produce multiple frequency response from a single input excitation frequency. In addition, these nonlinear systems are sensitive to initial conditions, and therefore the state is important. This is evidenced with the nonlinear jump phenomena responding with different jumps in amplitudes around the resonance depending on the direction of the ramp. The natural frequency calculated from linear interpolation of the equations around a specified amplitude range changes with the respect to frequency as the effective linear stiffness is changed due to the nonlinearity. All these characteristics of nonlinear systems are important for identifying the nonlinear system. 


\section{Dissertation Structure}

The dissertation will provide both experimental and analytical analysis. The driveshaft and test system will be introduced that defined the problem. Test data and analysis identifying nonlinear response of super harmonics of an automotive driveshaft system will be discussed. Subsequent measurements of the physical characteristics of the system will be provided including elastomeric stiffness measurements and linear system experimental modal analysis identifying the natural frequencies of the system. These measurements quantify both the system characteristics and the nonlinear problem being addressed.

The excitation due to the universal joint comprises a key input to replicating the system for analysis. A complete derivation identifying the dynamic input excitations with both with and without applied static moments will be presented. A Fourier series

will be developed to further understand the input excitation to the system. The inclusion of multiple super harmonic excitation of the system will be discussed.

The analytical portion will begin with the formation of an analytical model. A correlated system of equations will be developed replicating the details of the physical system. The correlation of both modal and simulation results replicating the empirical data will be presented and discussed. In compliance with the driveshaft system, the model will include the elastomeric support of the center bearing, elastomeric support of an internal tuned damper (ITD) and NCV joint. The analytical model will provide the basis for additional nonlinear analysis. 
Advanced nonlinear analysis techniques will be applied to the problem to determine stability and response characteristics that have not previously been applied to the problem. Bifurcation and Poincare techniques are applied to determine the stability and search for ranges of bifurcations leading to chaos. Lyapunov analysis will be used to determine if ranges of chaotic vibrations are possible. Spectral analysis will help identify components of chaotic vibrations including sub harmonics. Analysis leading to identification of multiple solutions in the nonlinear response range will also be presented. All of these contributions will provide significant insight to the nonlinear response characteristics and $\mathrm{NCV}$ joint dynamics in common vehicle driveshaft systems.

\section{Original Contributions of the Dissertation}

The work of the dissertation provides multiple significant contributions. The dissertation work is the first experimental confirmation of Superharmonic jump in a driveshaft system. The driveshaft system studied is based on an actual problem of observed objectionable noise in a vehicle. Significant experimental work was performed with the shaft system producing the noise, and replicated the issue's physical vibrations and audible noise. The dissertation shows how an Internal Tuned Damper (ITD) that was installed to attenuate unwanted vibrations, created other vibration problems. Subsequent analytical work was defined to replicate mathematically the physical test system. This approach is novel as compared to previous work, as the analysis was performed and a confirmation test if any was subsequently performed to prove the analysis. 
The analytical model, based on the physical shaft system, is significantly more complicated and therefore more detailed than previous analytical models. First, the applied secondary moment due to the universal joint misalignment is derived for the first time incorporating no static torque load on the shaft system. This once again replicates the test system to accurately apply to the analysis. Second, the analytical model is significant with complex physical characteristics of the automotive driveshaft with multiple shafts, multiple diameters of the driveshaft, universal joints, and a soft center bearing support. The dissertation is the first to consider an nonlinear ITD in addition to the nonlinear excitation by the NCV joint. Due to these complexities, a full FE model proved to have significant processing demand preventing subsequent timely simulation and analysis. Therefore, the dissertation provides the first application for simplified nonlinear mode development through modal coupling interfaces. This was accomplished by using modal reduction and coupling techniques applied to the nonlinear system before simulation and analysis.

Since novel model reduction techniques were applied to the nonlinear system, all subsequent nonlinear analysis using the reduced system also become significant in the contributions. This includes the bifurcation, Poincare, Lyapunov, and multiple solution analysis performed. The dissertation provides the first use of Lyapunov Exponents to confirm chaotic lateral vibration in a driveshaft system. Subsequently, the dissertation provides a set of conditions of chaotic vibrations in a driveshaft system.

Therefore, the work does provide significant contributions to the area of nonlinear vibrations, driveshaft system vibration analysis, understanding of NCV joint 
characteristics, significant experimental work, and practical application of the entire testing and analysis to a current noise and vibration issue. 


\section{CHAPTER II}

\section{DRIVELINE SYSTEM*}

The driveshaft of consideration is a commercially available driveshaft outfitted on a current production vehicle. The vehicle is an all wheel drive (AWD) that is based on a front wheel drive platform as pictured in Fig. 4. The vehicle therefore has a transversely mounted engine with a transaxle. A Power Transfer Unit (PTU) is attached to the transaxle to split torque to the rear axle, differential, through the driveshaft.

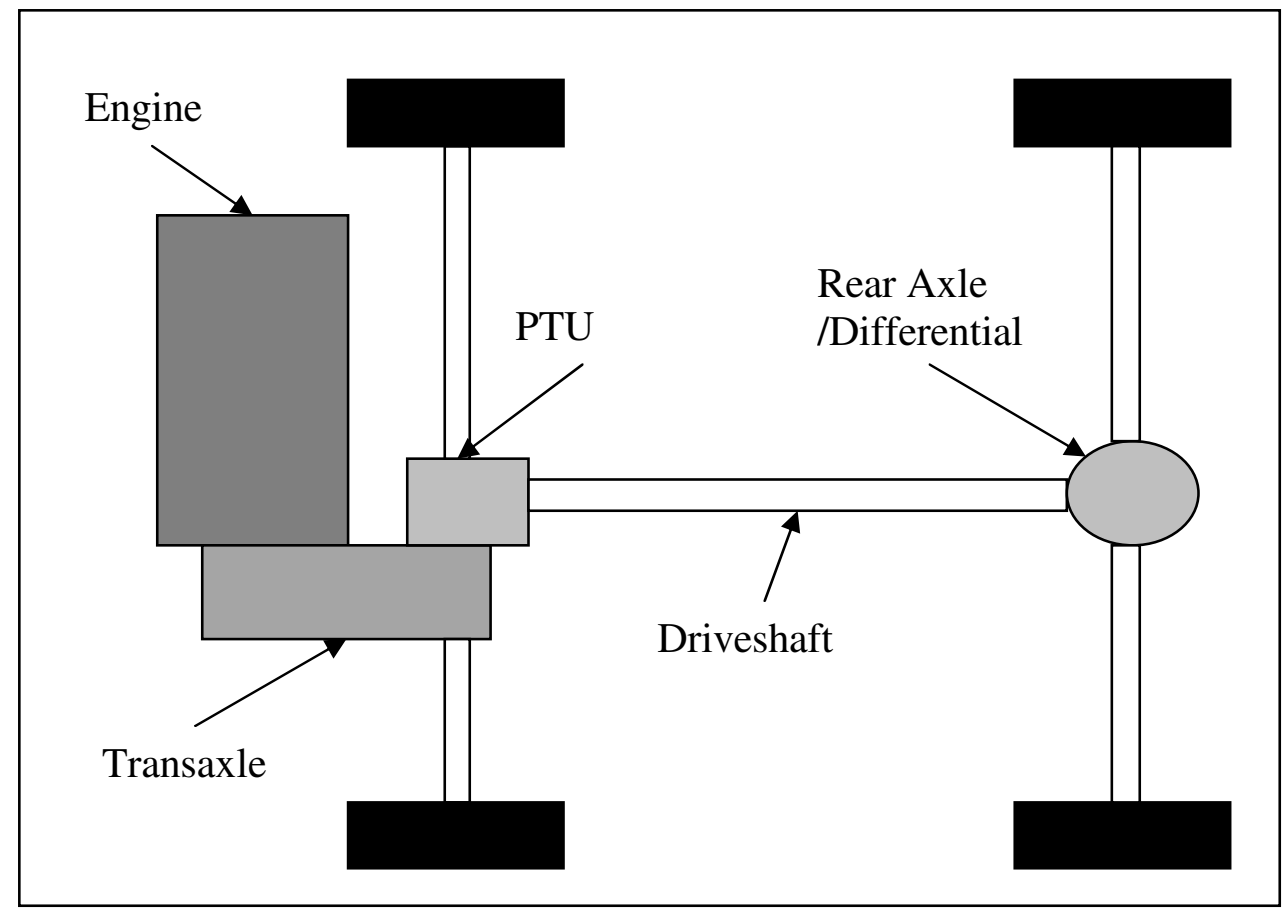

Fig. 4. All-wheel Drive Drivetrain Layout.

*Part of this chapter is reprinted with permission from "Superharmonic nonlinear lateral vibrations of a segmented driveline incorporating a tuned damper excited by nonconstant velocity joints" by M. Browne and A. Palazzolo, 2009. Journal of Sound and Vibration, doi:10.1016/j.jsv.2008.12.018. 
Driveshafts connecting the PTU to the rear axle differential assembly can be fabricated using many different shaft lengths and joint methods. The number of shafts is typically due to sixing the shafts and diameters so that the shaft can transfer the required torque loads and the necessity to avoid catastrophic critical speeds. These critical speeds are identified by the first bending mode of each of the driveshafts. The design of a driveshaft requires that the first bending mode(s) exceeds the first order excitation with a safety factor from the maximum vehicle speed. Joints are included in the shaft to allow for misalignment. Misalignment in a vehicle is due to many factors including manufacturing tolerances, packaging constraints, suspension travel, and engine dynamic movement (pitch and roll). Non-constant velocity (universal joint or Hooke's joint), constant velocity (CV joint), and flex couplings have been used in vehicle driveshaft applications for these reasons.

\section{Physical Description}

The driveshaft of study is of a two piece design incorporating a constant velocity joint, $\mathrm{CV}$ joint, at the transmission end of the shaft, and two non-constant velocity joints located in the center and rear axle end of the shaft as depicted in Fig. 5 and pictured in Fig. 6. 


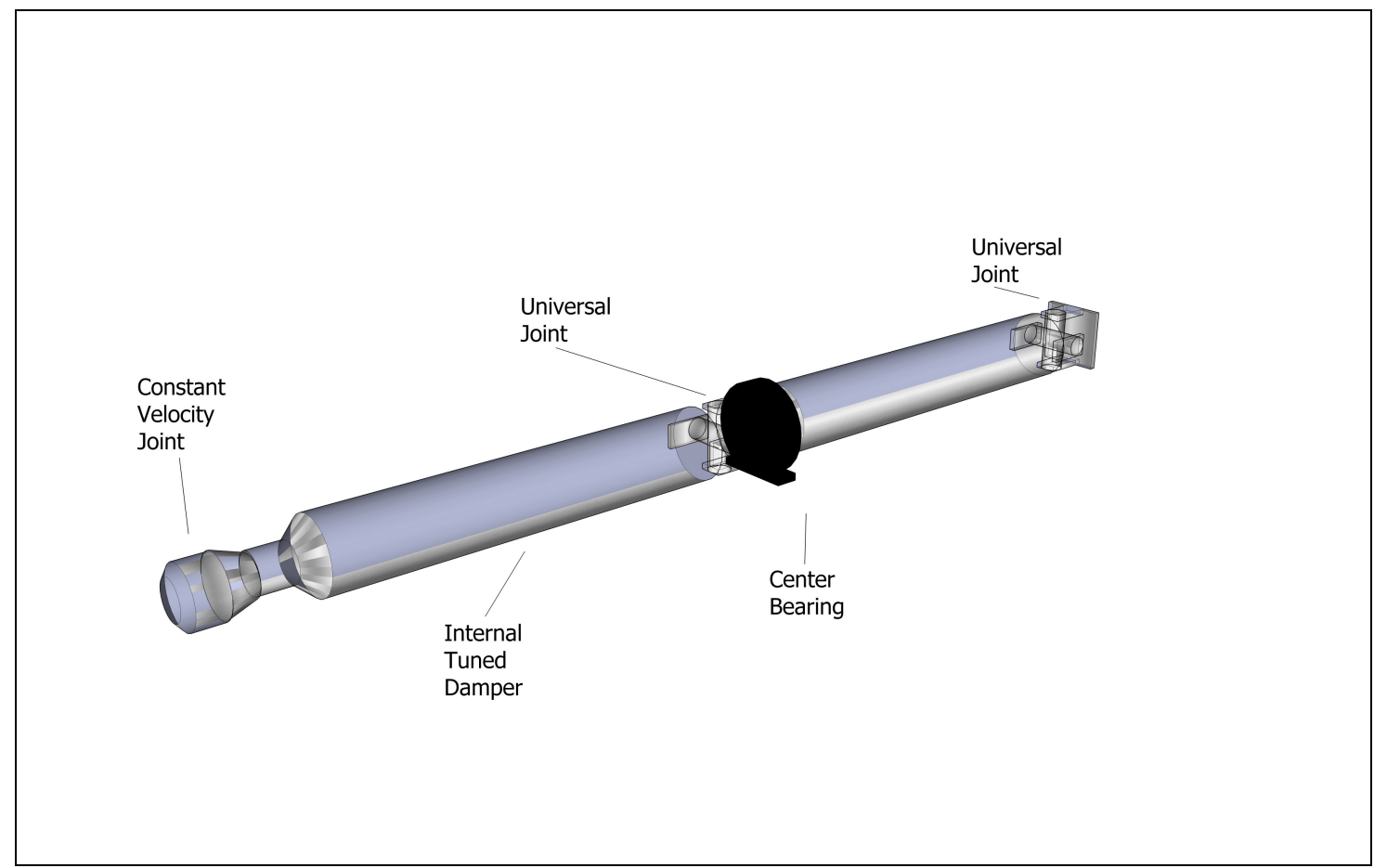

Fig. 5. Schematic of Driveshaft.

The constant velocity joint is of the plunging type with six balls allowing for the angular misalignment. The center bearing consists of a ball bearing supported by a diaphragm type flexible rubber bushing. The non-constant velocity joint is located just to the front of the center bearing assembly. The second non-constant velocity joint is located at the rear axle end of the driveshaft. In addition, a vibration absorber, internal tuned damper (ITD), is incorporated in the front shaft to minimize vibrations due to the first bending mode of the driveshaft. 


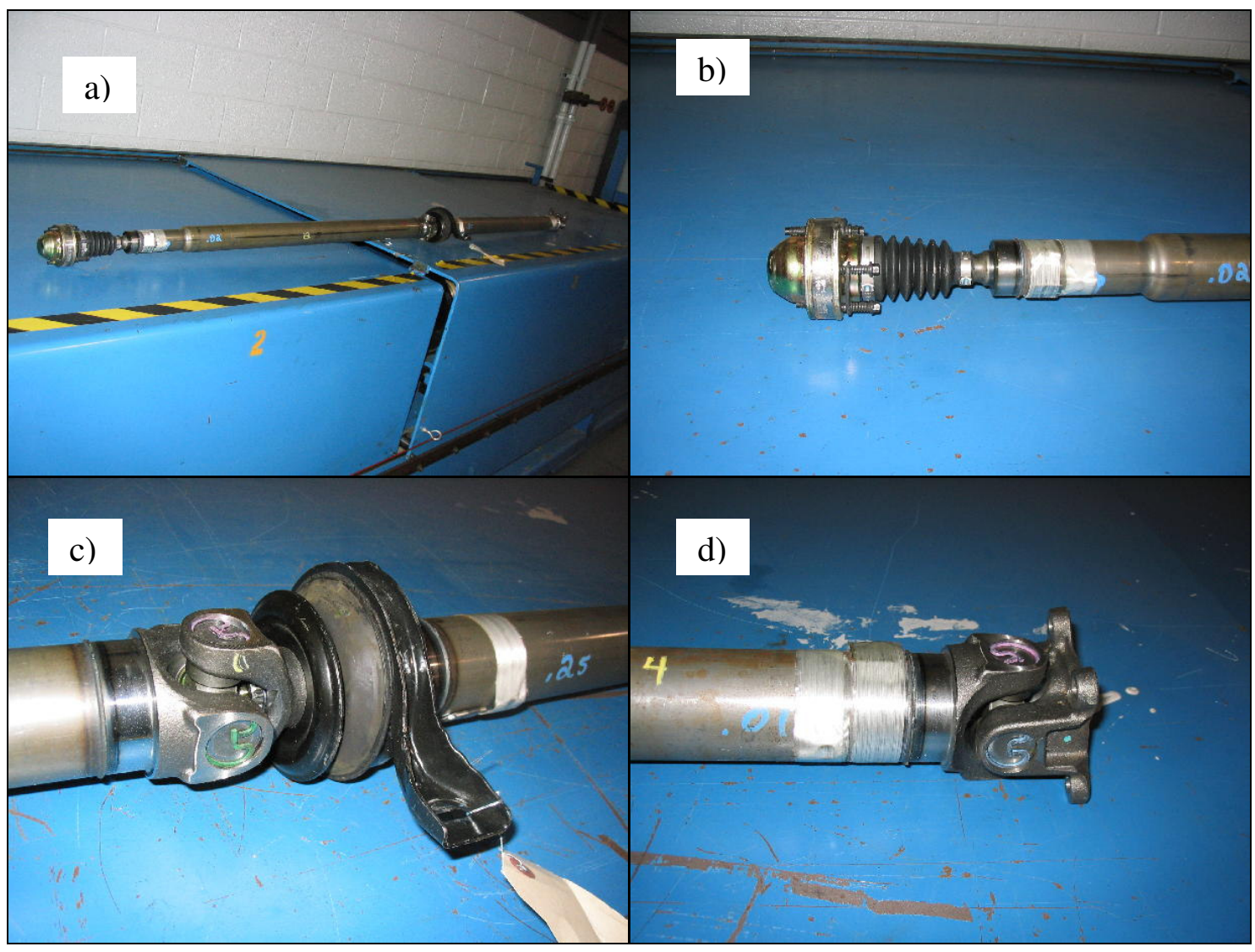

Fig. 6. a)Entire Driveshaft Assembly, b) Constant Velocity Joint at PTU/Transaxle End of Driveshaft, c) Center Bearing Assembly with Non=Constant Velocity Joint, and d) Axle End of Driveshaft Assembly.

\section{Test System Description}

The test bench is made up of a hydraulic motor that rotates the shaft on the transmission side, and a spindle at the opposite end to support the axle side of the driveshaft as shown in Fig. 7 and support for the center bearing in the middle. This support was designed to adjust the height of the center bearing. This adjustment is used to test the driveshaft with different universal joint angles by changing the height of the center bearing. 


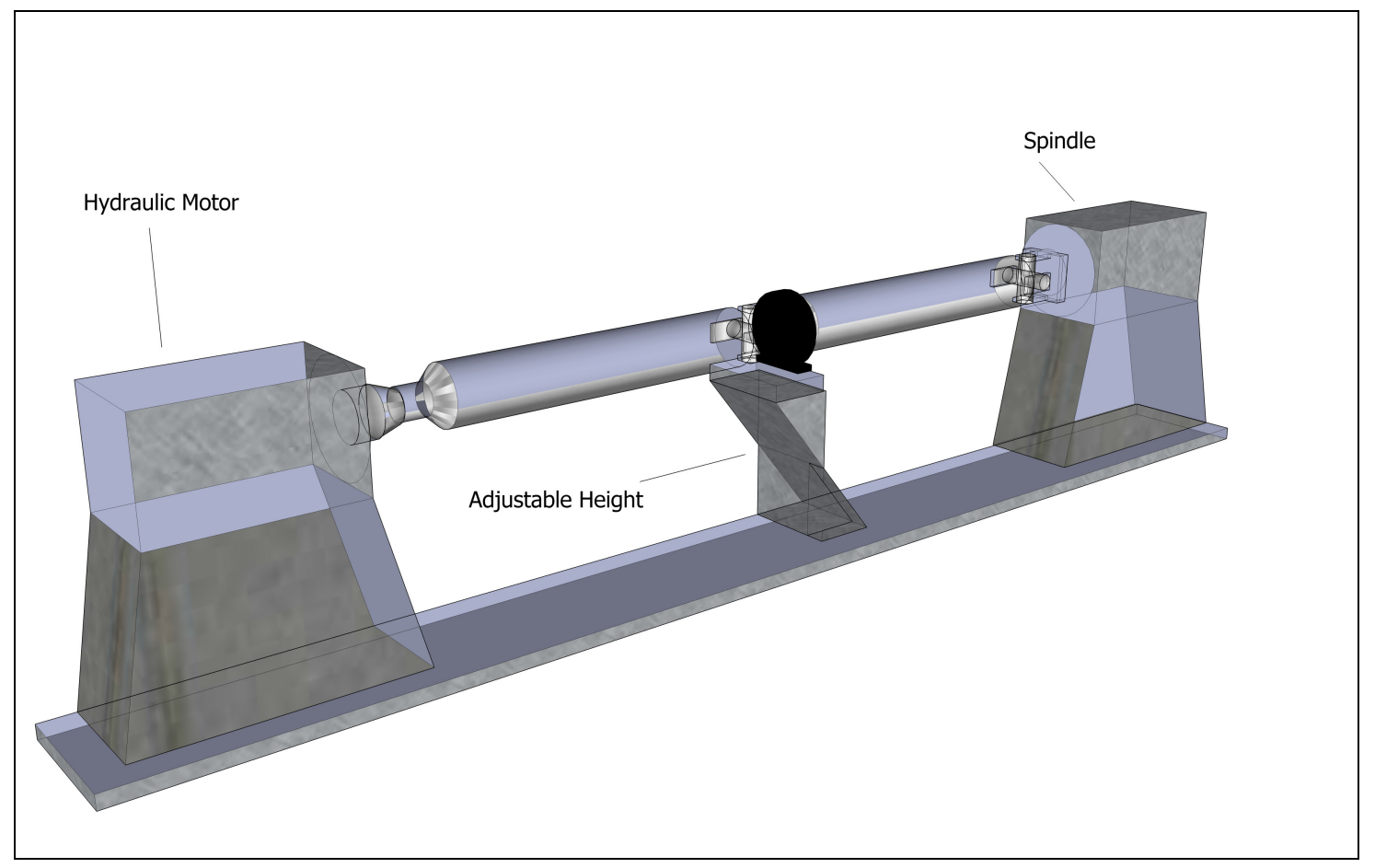

Fig. 7. Schematic of Test Stand.

It is significant as the system has no brake or secondary motor to provide additional torque. Therefore, the "load" torque in the shaft system is primarily due to inertia. This fixture has the capability of testing the driveshaft through a range of speeds. The test fixture ramps the driveshaft's rotational speed up and down through a typical vehicle's speed range of operation. Data acquisition equipment records the speed and vibration response data for analysis. For example, some measurement sensors are pictured in Fig. 8. 


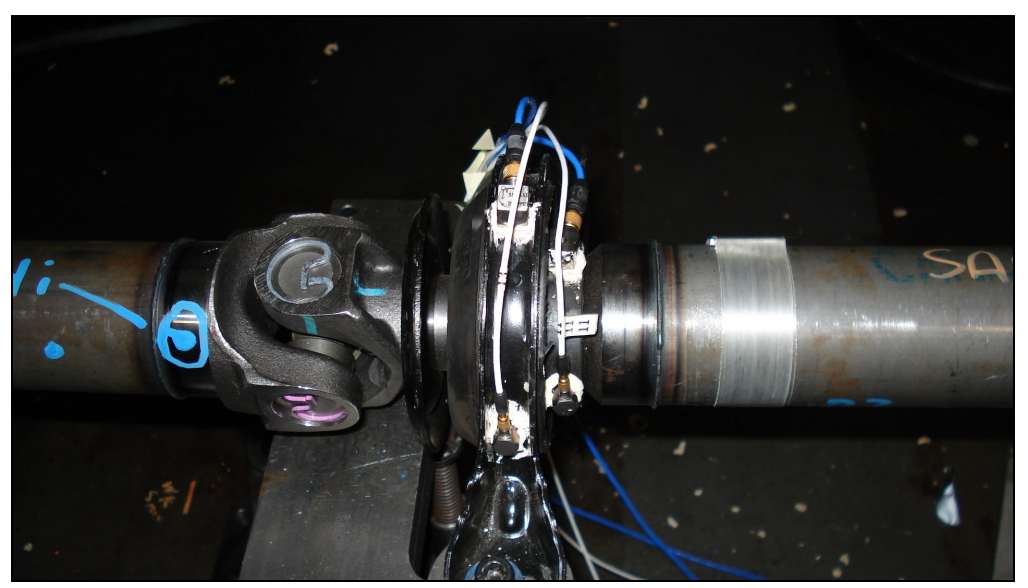

Fig. 8. Test Rig Instrumentation on Center Bearing.

Rotational speed data is collected through use of a tachometer on the axle end of the driveshaft. Vibration data is measured at accelerometers located on the transmission spindle, axle spindle, center-bearing bracket, and on the center bearing. The PTU/transaxle and axle accelerometers are tri-axis accelerometers that measure vibrations in all three principle directions. The accelerometers at the center bearing include two pairs of accelerometers: a tri-axis accelerometer on top and a single axis accelerometer on the side. One accelerometer set is located on the passive (grounded) and active (driveshaft) sides of the center bearing support mount.

The test stand provides a means to test the driveshaft, removed from the more complex vehicle environment. The stand allows for various test conditions of various speeds and NCV joint misalignments. In addition, the measurements allow for significant vibration measurements on the non-rotating bearing support locations of the driveshaft. 


\section{Experimental Results}

Two sets of experiments are required to understand the physical characteristics of the system including the operating response, and the system's physical dynamic characteristics. The operating response identifies the unique vibration response of the system. The subsequent testing to define the physical dynamic characteristics includes an experimental modal analysis to determine the natural frequencies for correlation, and stiffness measurements of the elastomeric components of the ITD and the center bearing support.

\section{Operating Response}

Multiple tests were performed characterizing the operating response of the driveshaft in the test system. The majority of tests were performed using a run-up and run-down speed profile. The speed was ran from stop to approximately 3600 RPM, and then run back down until the shaft was stopped. Thirty five seconds was allotted for the run-up and an additional thirty five seconds to the run-down. Therefore, a consistent ramp rate was used for both the run-up and run-down portions of the testing. This profile aids in identifying transient responses. Subsequent testing showed that at any point, the steady state continues with the levels during the transient portion, and therefore subsequent steady state testing was unnecessary.

A non-constant velocity joint creates harmonic and super harmonic excitation on a driveshaft. The driveshaft response jumps during a ramp-up due to a nonlinearity in the systems. This response is most significant at the active side of the soft support of the 
center bearing of the driveshaft. Measurements of the lateral acceleration on the soft support are presented in Fig. 9 for a run-up, run-down, and a run up with no driveshaft conditions.

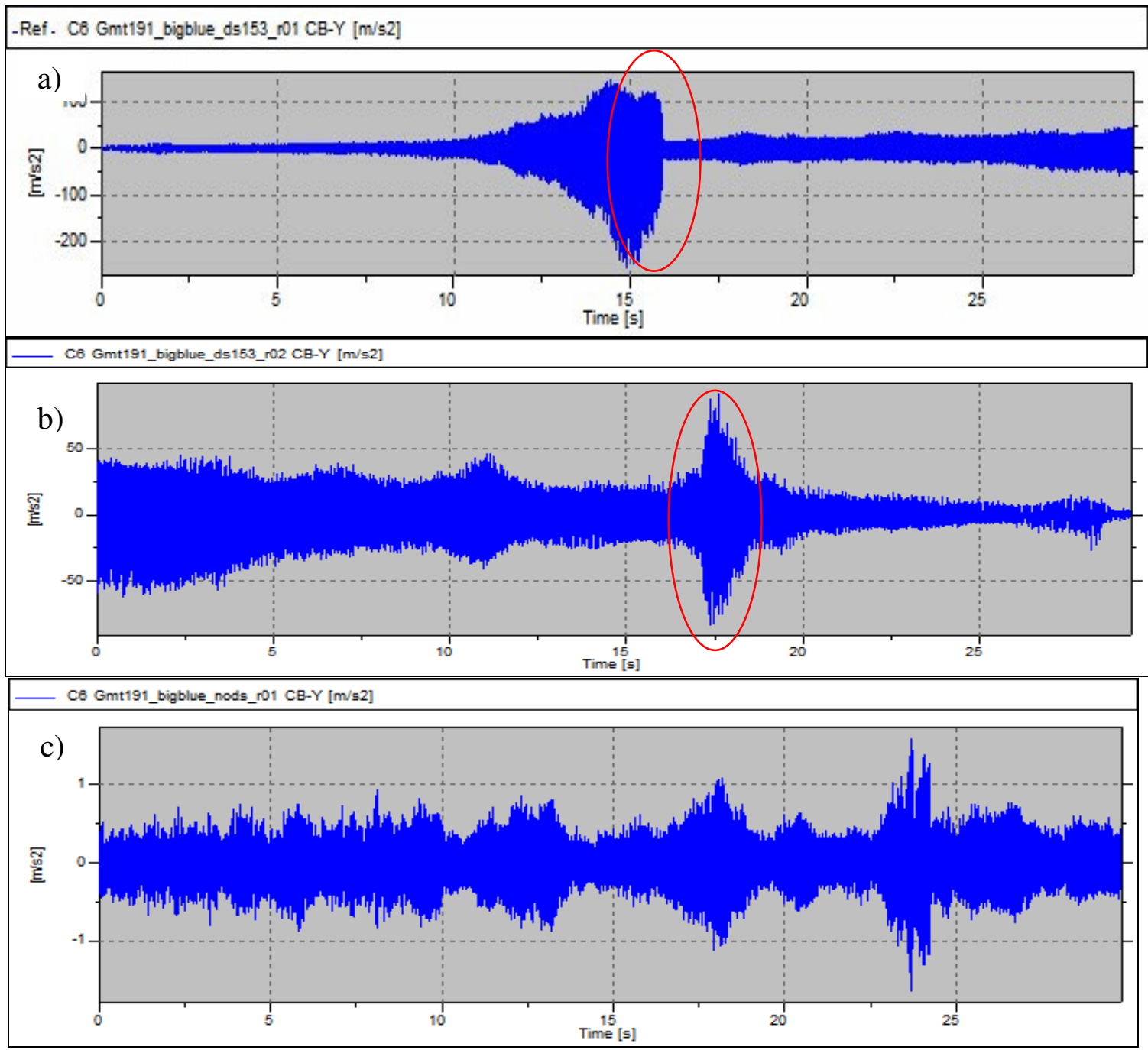

Fig. 9. Lateral Acceleration Measured at the Active Side of the Center Bearing during a) Run Up, b) Run Down, and c) Run Up with no Driveshaft. 
The time data shows a significant reduction of vibration during run-up at approximately 16 seconds. During the run down a ramped increase of vibration is seen at approximately 17 seconds into the run down. The ramp rates are constant, and therefore the peak of the high vibration areas are at a lower frequency during run-down than during the run-up. In addition, the peak amplitude of the run-up $(\sim 200 \mathrm{~m} / \mathrm{s} 2)$ is significantly higher approximately than the run-down $(\sim 80 \mathrm{~m} / \mathrm{s} 2)$. In addition, the runup with only the rotation of the motor did not produce significant vibrations, thus eliminating the hydraulic motor as the source of significant vibration with less than 1 $\mathrm{m} / \mathrm{s} 2$ vibration levels throughout the run-up. These results indicate the possibility of nonlinear jump response.

Spectral analysis of the data identifies the primary harmonics comprising the excitations of the jump are the second, fourth and eighth order rotational. A contour plot of the order spectrum is provided in Fig. 10. 


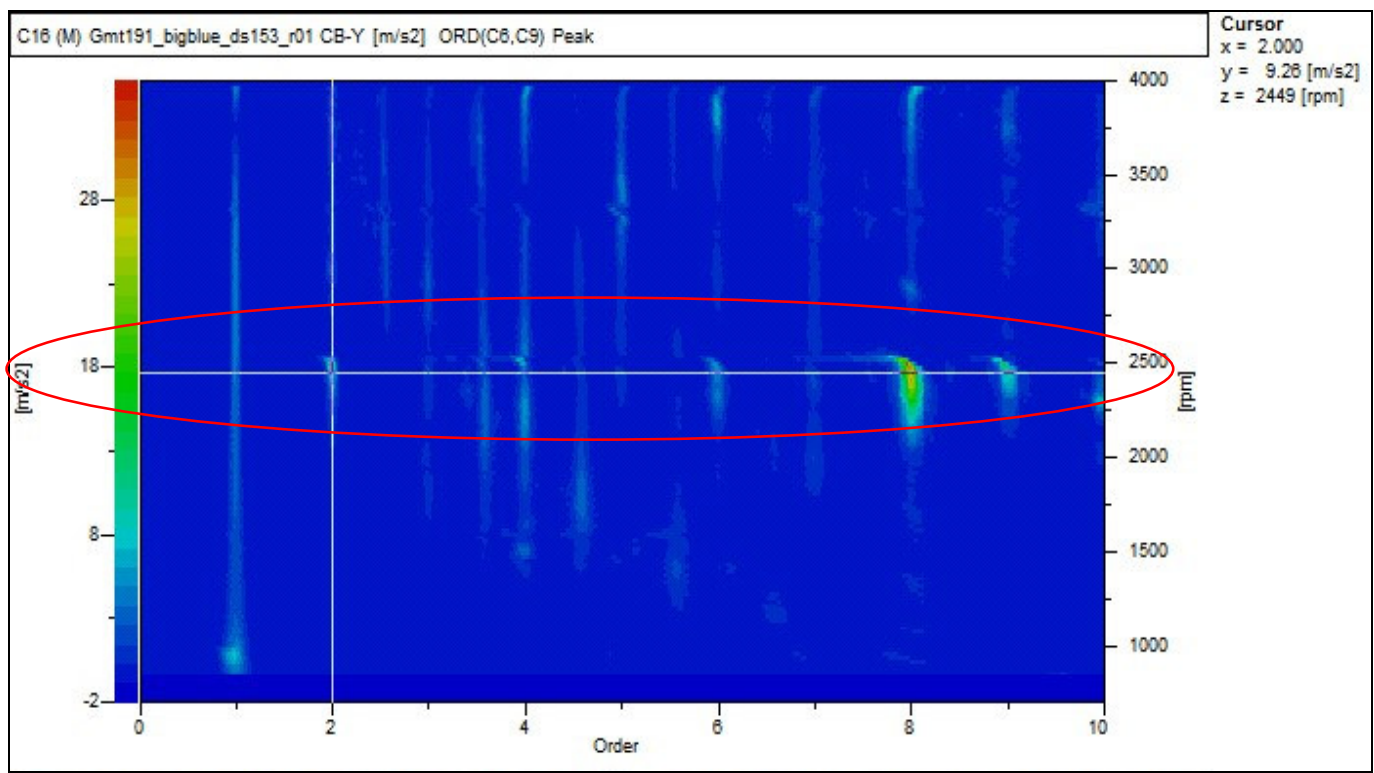

Fig. 10. Run Up Order Spectrum of Lateral Vibration at Active Side of the Center Bearing.

This jump follows typical stiffening nonlinear characteristics, as the jump is significant on a run up portion of a test and minimal and at a lower rpm (frequency) during the run-down portion of the test cycle. The jump is interesting as it occurs not in the primary rotational order, but in the harmonics of the rotational including $2^{\text {nd }}, 4^{\text {th }}$, and $8^{\text {th }}$ orders. This is significant, as the complaints of noise in vehicle were identified as from the fourth and eighth order rotational harmonics.

From the initial testing, two setup factors were identified as significant contributors of driveshaft vibrations. These are the NCV joint angle and imbalance. To further understand the system, excitation and responses, these two factors are tested at multiple levels to understand the vibration response due to each factor and their interactions. Therefore, the test setups include 0,2 , and 4 degrees of angle across the 
center NCV joint, and four imbalance levels per angle setup. The shaft was initially

balanced, and balance weights of $0,4,13$ and 25 grams were added to the surface of the shaft at the universal joint to add imbalance to the system. Furthermore, three repetitions per imbalance and angle setup are recorded for analysis to quantify the test and measurement repeatability. As a Design of Experiments (DOE) was outlined for comparing multiple levels of the factors, a complete evaluation of the test setup and fixtures ensured proper installation and all bolted joints were properly torque to insure good measurements for comparison.

NCV Misalignment Results

From all the testing results, comparing the repetitions at each imbalance level across the range of NCV joint misalignment produced similar results. Therefore, a comparison of the angles with the driveshaft system in the balanced condition is presented. A comparison of the time response of the system at 0 (red) and 4 degree (blue) misalignment angle at the center bearing active side is plotted in Fig. 11. 


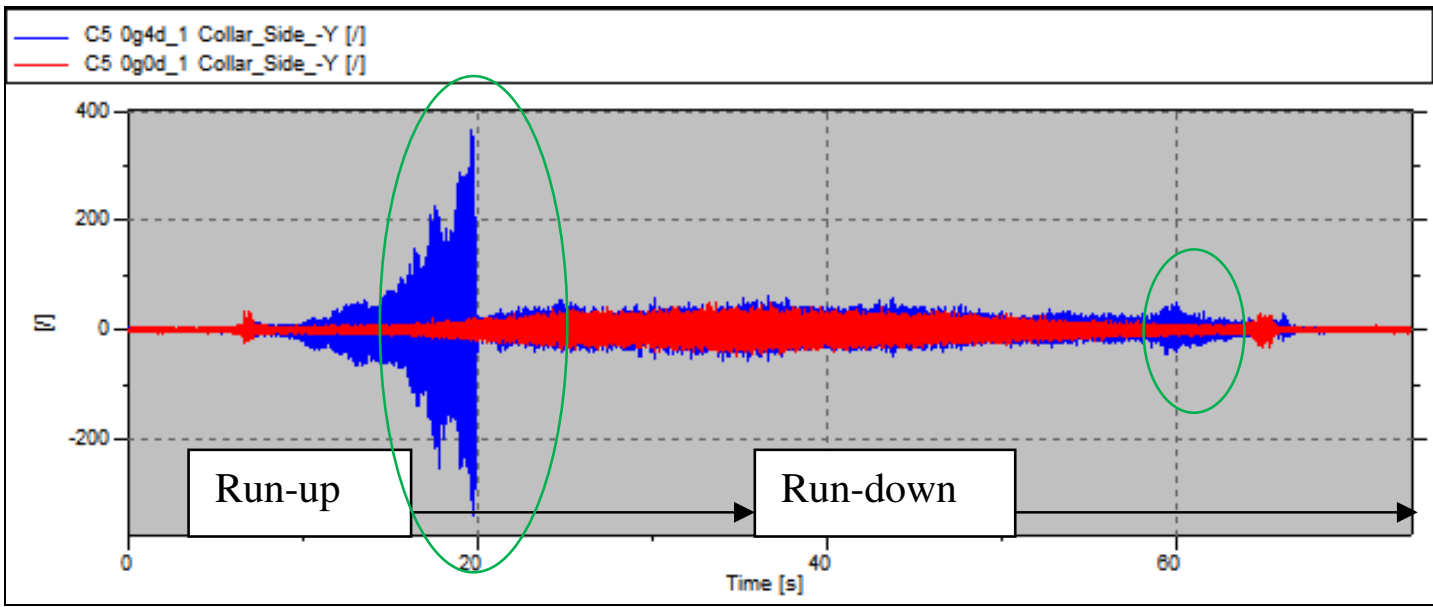

Fig. 11. Measured Acceleration at Center Bearing Active Side of Support Mount at 0 (red) and 4 Degrees (blue) of Misalignment.

The time response clearly shows a distinct difference between the two misalignment angles. There is a distinct increase of vibration levels from 10 to 20 seconds during the run-up portion of the test. In addition, the previously identified jump is apparent at 20 seconds into the test cycle. The amplitude of almost $400 \mathrm{~m} / \mathrm{s} 2$ before the jump is at a significant level of vibration. The level is higher than the initial screening test as the angle of 4 degrees is larger than the original test. In addition, the same trend with little increase of vibration occurring during run-down corresponding with the increased vibration in the run up is seen at approximately 60 seconds. This shows a lower frequency of the increased vibration on the run-down as in the run-up. The time data verifies the nonlinear jump response in the time domain for 4 degrees of misalignment, but not with any misalignment. 
Subsequent processing of the data reveals the order content of the two misalignment angles. Contour maps of the vibration order spectrums for both the 0 degree and 4 degree misalignments are presented in Fig. 12.
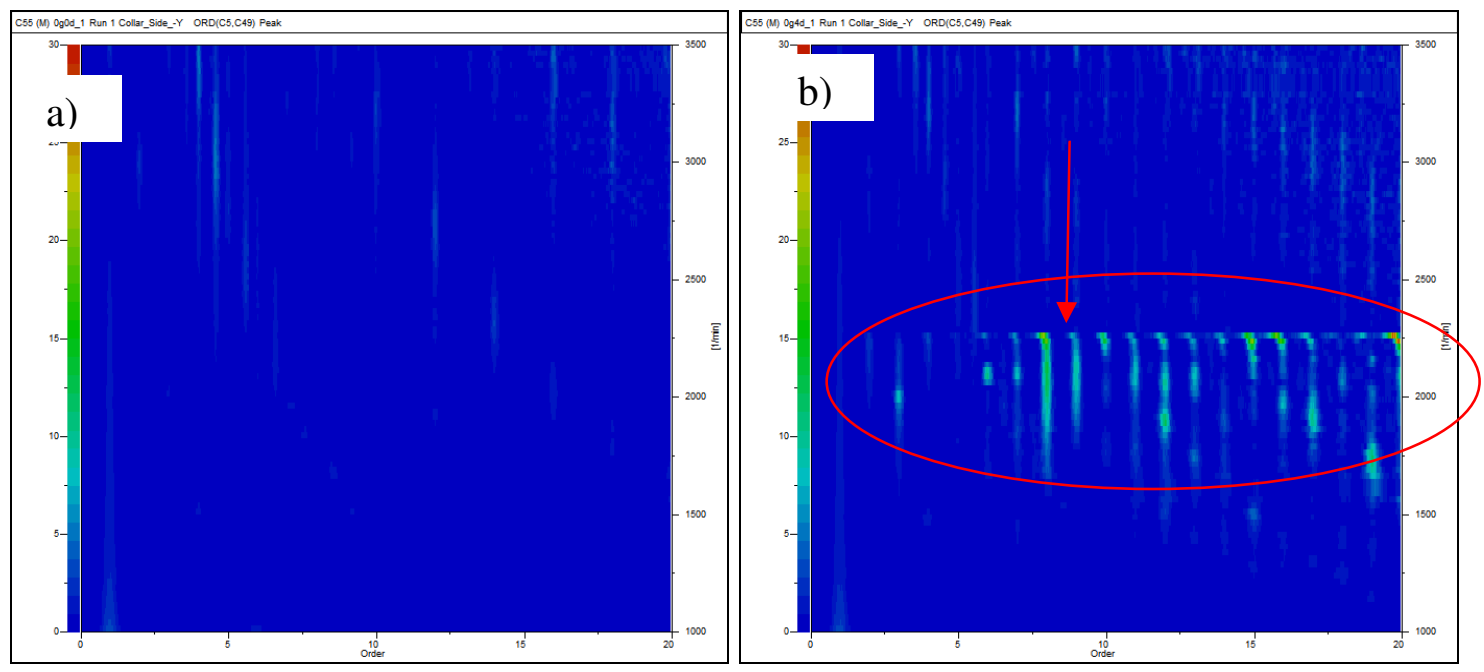

Fig. 12. Order Spectrum for Run Up for a) 0 Degrees of Misalignment and b) 4 Degrees of Misalignment.

Significant integer order excitations are noted in the vibration spectrums in the 4 degree, but are not present in the 0 degree of misalignment. This corresponds to the increased vibrations in the tome domain previously noted All of the integer orders above $1^{\text {st }}$ order show to some degree the jump of vibration levels at approximately 2250 RPM. The most significant acceleration order appears to be the $8^{\text {th }}$ order. It is also interesting to note that at the jump, the vibrations veer away from tracking the order, but follow the constant frequency line for a short period of time before the vibrations cease. 
Due to the NCV joint excitations of the even harmonics, the $2^{\text {nd }}, 4^{\text {th }}, 6^{\text {th }}$, and $8^{\text {th }}$ order cuts from the spectrum data is presented in Fig. 13 for all three misalignment angles of 0 (blue), 2 (red), and 4 (magenta) degrees of misalignment.
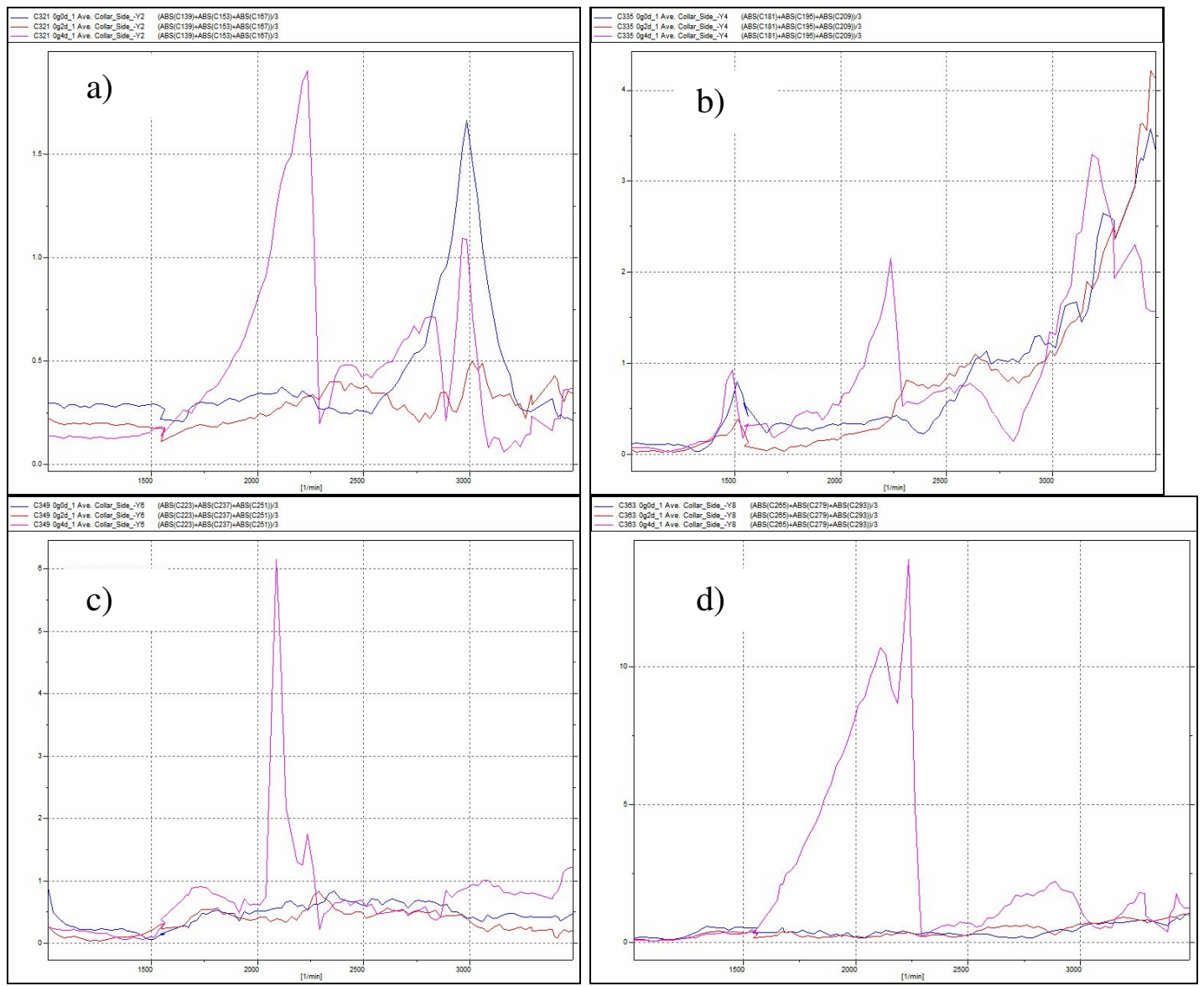

Fig. 13. Order Comparison for a) 2nd Order, b) 4th Order, c) 6th Order, and d) 8th Order with Misalignment Angles of 0 (blue), 2(red), and 4 Degrees (magenta).

The jump at approximately 2250 RPM is clearly observed in the $2^{\text {nd }}, 4^{\text {th }}$, and $8^{\text {th }}$ harmonics in only the 4 degree of misalignment condition. The $6^{\text {th }}$ order has increased vibration in the range of the jump, but does not show the jump in levels as the other 
vibration. From the 4 orders, the $8^{\text {th }}$ order has the highest acceleration levels, but the $4^{\text {th }}$ order has the highest amplitude when integrated to displacement. Therefore, the misalignment angle is a significant factor influencing the nonlinear jump in the experiment.

Imbalance Level Results

The factor of imbalance can be analyzed similar to the misalignment angle. Choosing a misalignment angle, comparing the results of the various imbalance levels will reveal any influence of imbalance on the system response. A misalignment of 4 degrees was chosen to determine the effects of imbalance on the nonlinear jump as it was the only angle producing the jump. Imbalance produced a harmonic, $1^{\text {st }}$ order, vibration response from the system. Therefore, the $1^{\text {st }}$ order response plotted in Fig. 14 shows the differenced of 0 (blue), 4 (red), 13 (magenta), and 25 grams (yellow) of added imbalance at the center universal joint. 


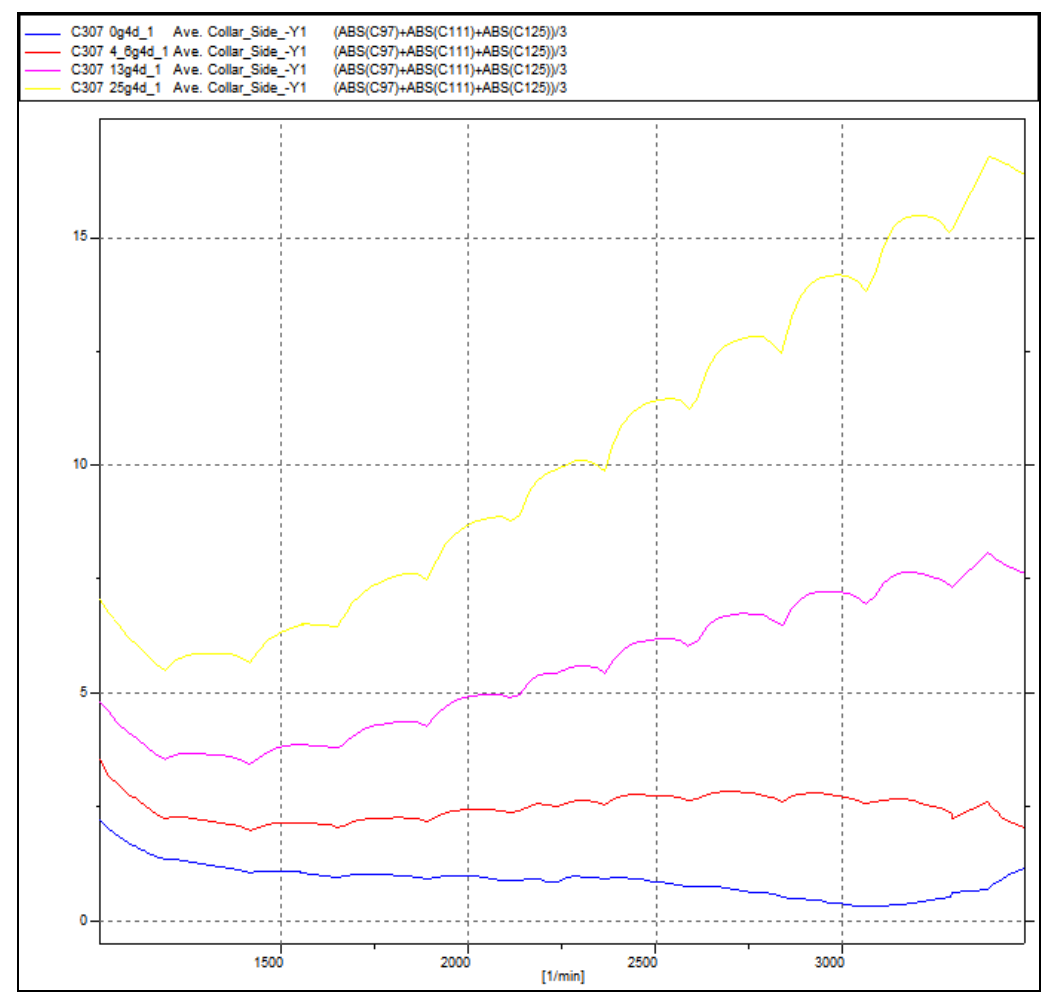

Fig. 14. 1st Order Response at 4 Degrees of Misalignment Due to Imbalance with Added 0 (blue), 4 (red), 13 (magenta), and 25 Grams (yellow).

The increased imbalance due to the added mass on the driveshaft at the center NCV joint increased the $1^{\text {st }}$ order vibrations as expected. The vibration levels are roughly proportional to the added amount of mass creating the imbalance.

A comparison of the order spectrum will help reveal any changes to the order content of the jump with different imbalance levels. Spectrum comparison at the 4 degrees of misalignment for the balanced and 25 grams of added imbalance are presented in contour plots in Fig. 15. 


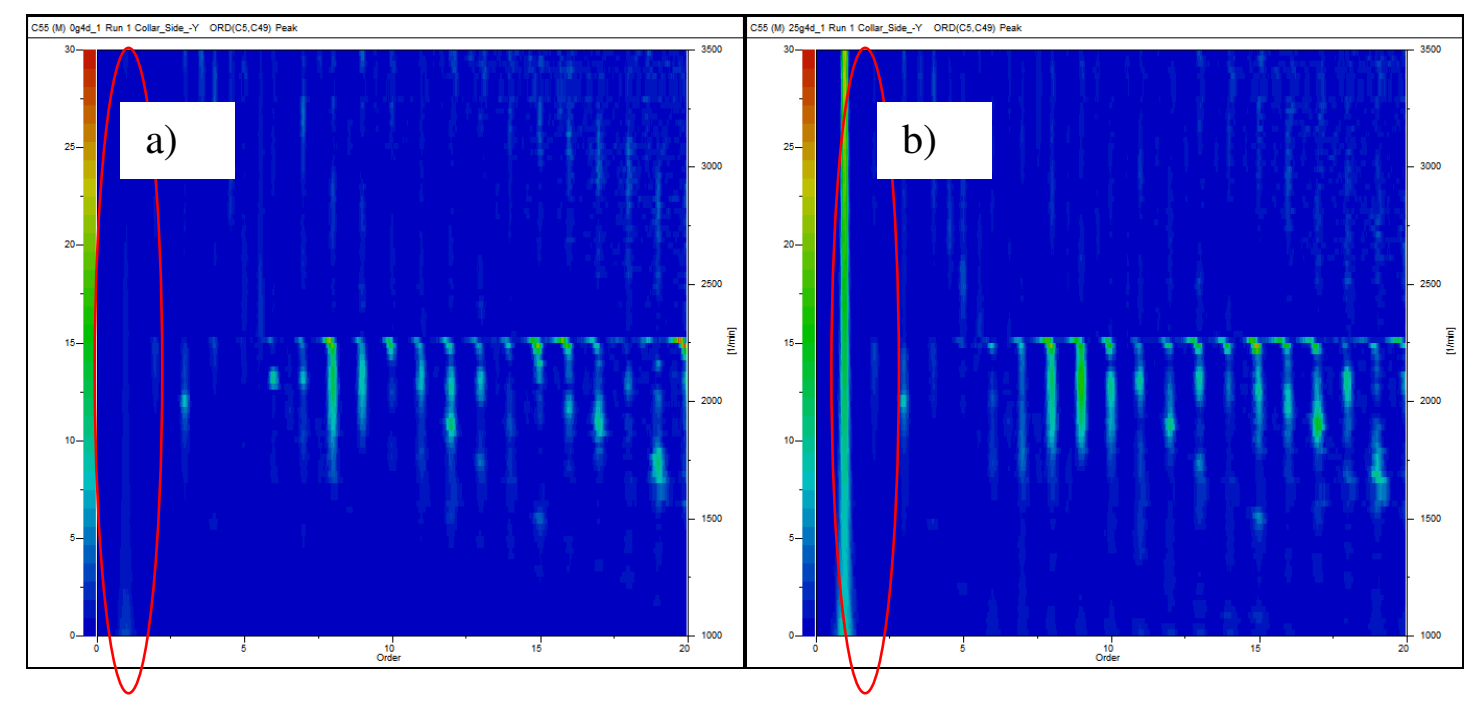

Fig. 15. Comparison of Order Spectrum Response with 4 Degrees of Misalignment for a) 0 and b) 25 Grams.

The first order response in the order spectrum shows a significant increase of vibration levels as expected from the $1^{\text {st }}$ order plots. Otherwise, the order spectrums reveal no significant differences between the balanced and the high unbalanced conditions. Further comparisons of the $2^{\text {nd }}, 4^{\text {th }}, 6^{\text {th }}$, and $8^{\text {th }}$ orders are shown in Fig. 16. 


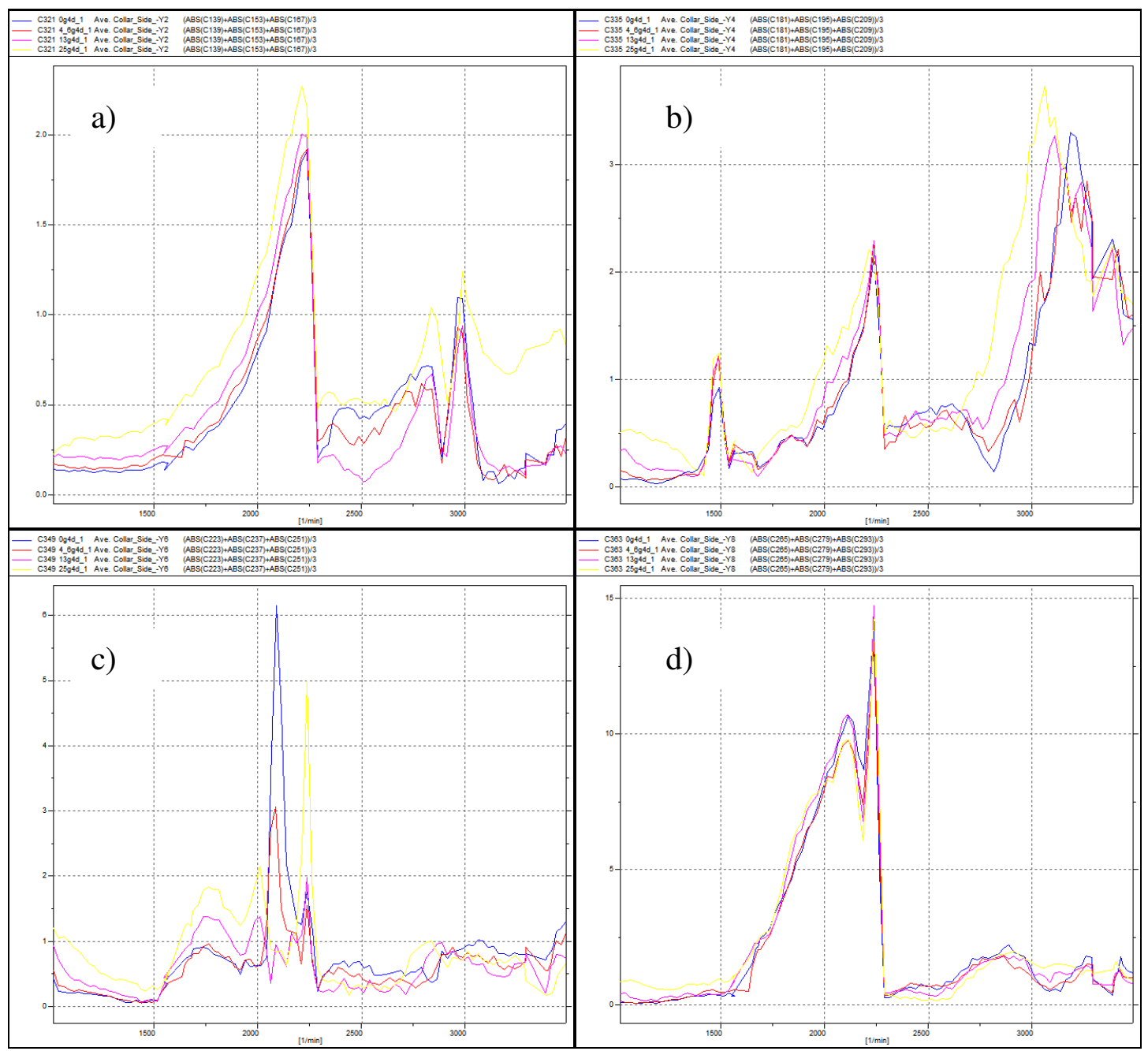

Fig. 16. Order Response at Multiple Imbalance Levels a) $2^{\text {nd }}$ Order, b) $4^{\text {th }}$ Order, c) $6^{\text {th }}$ Order, and d) $8^{\text {th }}$ Order at Imbalance of Added 0 (blue), 4 (red), 13 (magenta), and 25 Grams (yellow).

The $2^{\text {nd }}, 4^{\text {th }}$, and $8^{\text {th }}$ order does not show any significant differences due to imbalance. The jump occurs in the exact same speed no matter what imbalance level was applied to the driveshaft. The general trends remain for all three orders. The only difference is that $2^{\text {nd }}$ order shows increased levels at higher than 3000 RPM with at 25 grams of added imbalance. The $6^{\text {th }}$ order shows a difference between imbalance levels, 
but with no coherent trend with respect to imbalance. The $6^{\text {th }}$ order randomness may be due to repeatability.

Repeatability

Confidence in the previous results relies on the repeatability of the measured data. Since each combination of imbalance and misalignment angle measured three repetitions, a comparison of the runs will reveal the repeatability of the system vibration and measured response. Similar to the other factors, a common basis of a selected imbalance and misalignment angle will be chosen. Other factor combinations produced similar results, but the data presented is reduced to simplify the comparison.

Selected plots of order cuts reveal the repeatability of the measurements presented in Fig. 17. The plots present 3 measured order responses in colored solid lines, and the average with a black dotted line. With an imbalance of 0 grams and 0 degrees of misalignment, the $1^{\text {st }}$ and $2^{\text {nd }}$ orders are presented in Figs. 17 a-b. Subsequent repetitions are presented in Figs. 17 c-d for 25 grams of added imbalance and 4 degrees of misalignment. 


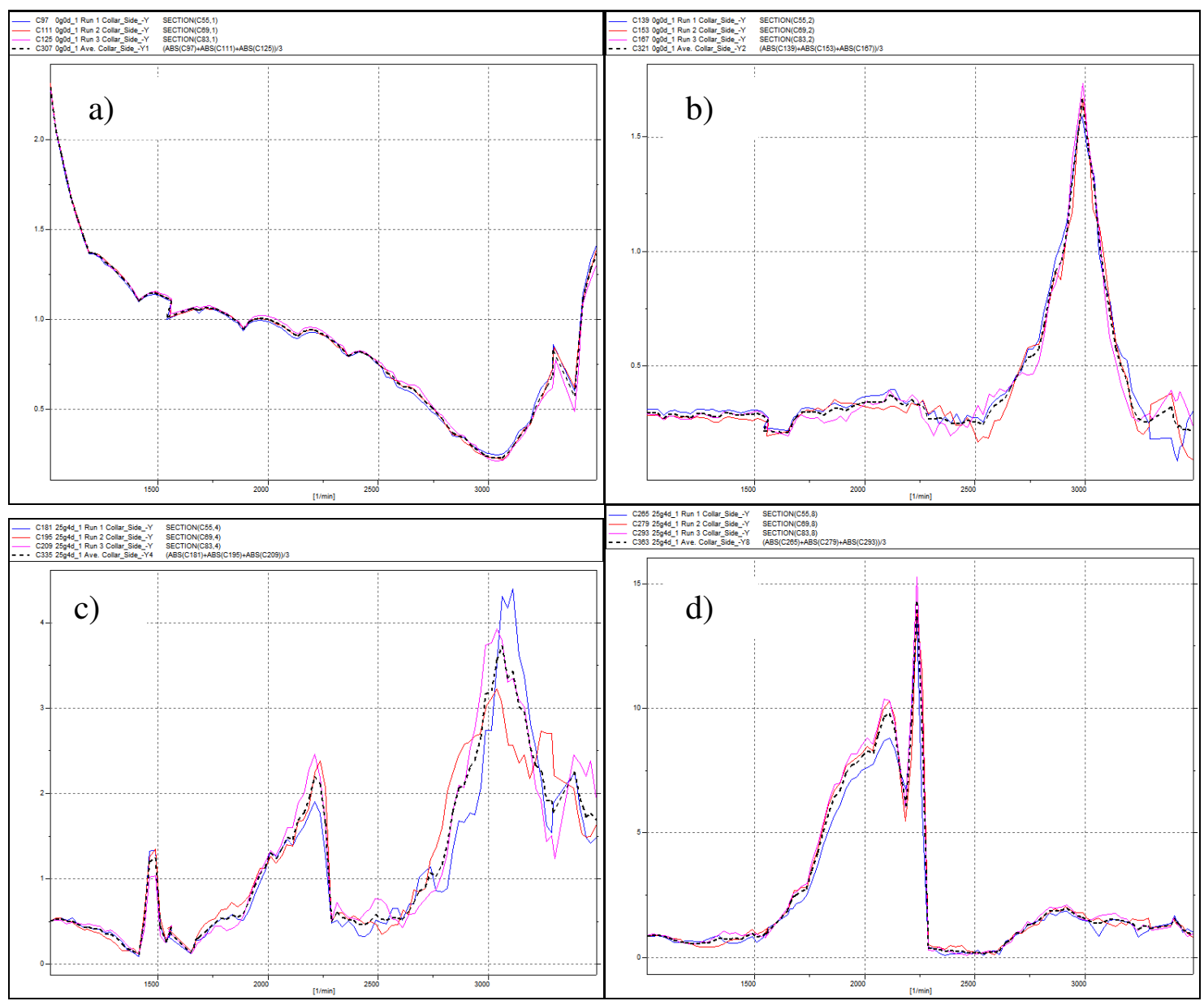

Fig. 17. Repeatability of Test Measurements at 0 Grams and 0 Degrees for a) 1st Order, b) 2nd Order, and 25 Grams and 4 Degree Misalignment for c) 4th Order, and d) 8th Order.

From the imbalance measurement, the $6^{\text {th }}$ order showed no consistent or correlated response with increased imbalance levels. This inconsistency of trend and level brought up the possibility of repeatability. Therefore, the $6^{\text {th }}$ order response with 0 degrees of misalignment and 0 grams of added imbalance is plotted in Fig. 18. Once again, the colored lines are individual measurement repetitions, while the dotted black line presents the average for all three measurements. 


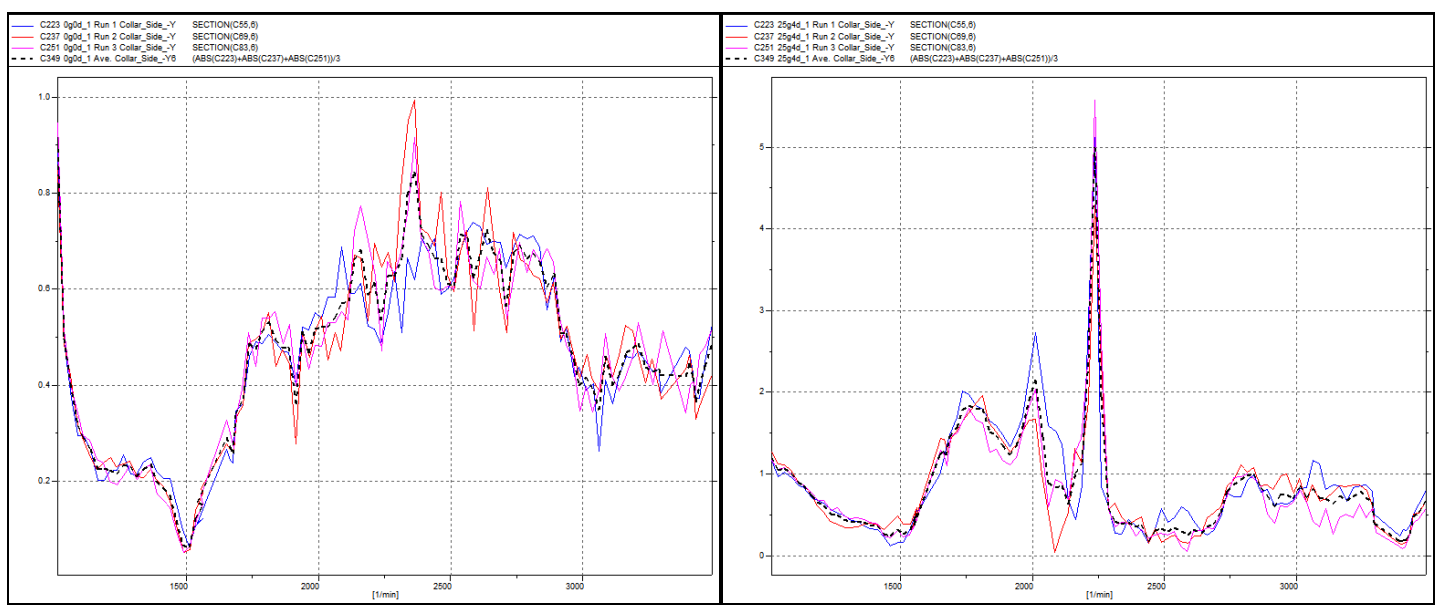

Fig. 18. 6th Order Repeatability Response at 0 Grams of Added Imbalance with a) 0 Degrees and b) 4 Degrees Misalignment.

The $6^{\text {th }}$ order response reveals significant increased variation of measurements, especially at the aligned measured response. The variation does not account for the total measured variation comparing the various imbalance levels, and therefore for $6^{\text {th }}$ order there may be some interaction between misalignment and imbalance levels for this order. The $6^{\text {th }}$ order has significantly lower levels of vibration, and does to show the nonlinear jump, the difference will not be investigated. Therefore, with the decreased correlation of the $6^{\text {th }}$ order, it will be shown that the excitation of the NCV joint are significantly lower for this order, and therefore is not a significant contributor to the nonlinear response.

\section{Physical Dynamic Characteristics}

In addition to the system response, physical characterization of the driveshaft and dynamic response are required to be quantified for accurate model definition This 
includes the natural frequencies of the test setup. The natural frequencies of the test setup are being vital to model the system correctly. Typical experimental modal analysis identifies the natural frequencies through linear analysis techniques. In addition to the experimental modal analysis, other necessary measurements include the weights, materials, and other dimensional characteristics of the driveshaft. Finally, the characterization of the known nonlinearities of the elastomeric support of the center bearing and internal tuned damper (ITS) is vital to accurate modeling. The force/deflection characteristics of the center bearing and ITD will be measured and quantified. These empirical tasks provide accurate information for modeling the system analytically.

\section{Experimental Modal Analysis}

Measurement of the natural frequencies of the test setup will be vital to model the system correctly. An experimental modal analysis of the driveshaft in the test fixture was completed and the responses curve fitted to obtain the natural frequencies, modal damping, and the mode shapes. Standard industry practices for experimental modal analysis were completed using LMS CADA-X software for both the data acquisition and analysis. A list of the mode frequencies less than $500 \mathrm{~Hz}$ is presented in Table 1 . These frequencies are identified as critical to creating a model that accurately represents the physical system. Therefore, the analytical system model has to have natural frequencies near these measured values and similar mode shapes. 
Table 1

Experimental Identification of Driveshaft Natural Frequencies Mode

Center bearing bounce

Front Driveshaft/ITD lower

Front Driveshaft/ITD upper

Rear driveshaft bending

$16.5 \mathrm{~Hz}$

$95 \mathrm{~Hz}$

$146 \mathrm{~Hz}$

Front driveshaft second bending

$162 \mathrm{~Hz}$

$385 \mathrm{~Hz}$

Each natural frequency has a corresponding mode shape. A description of the mode shape does not clearly portray the how the system is flexing. Therefore, each mode shape will be presented that was used for correlation to the analytical model. The center bearing bounce mode is pictured in Fig. 19. All components of the system are labeled for reference, and all subsequent pictures use the same colors and position for all the components.

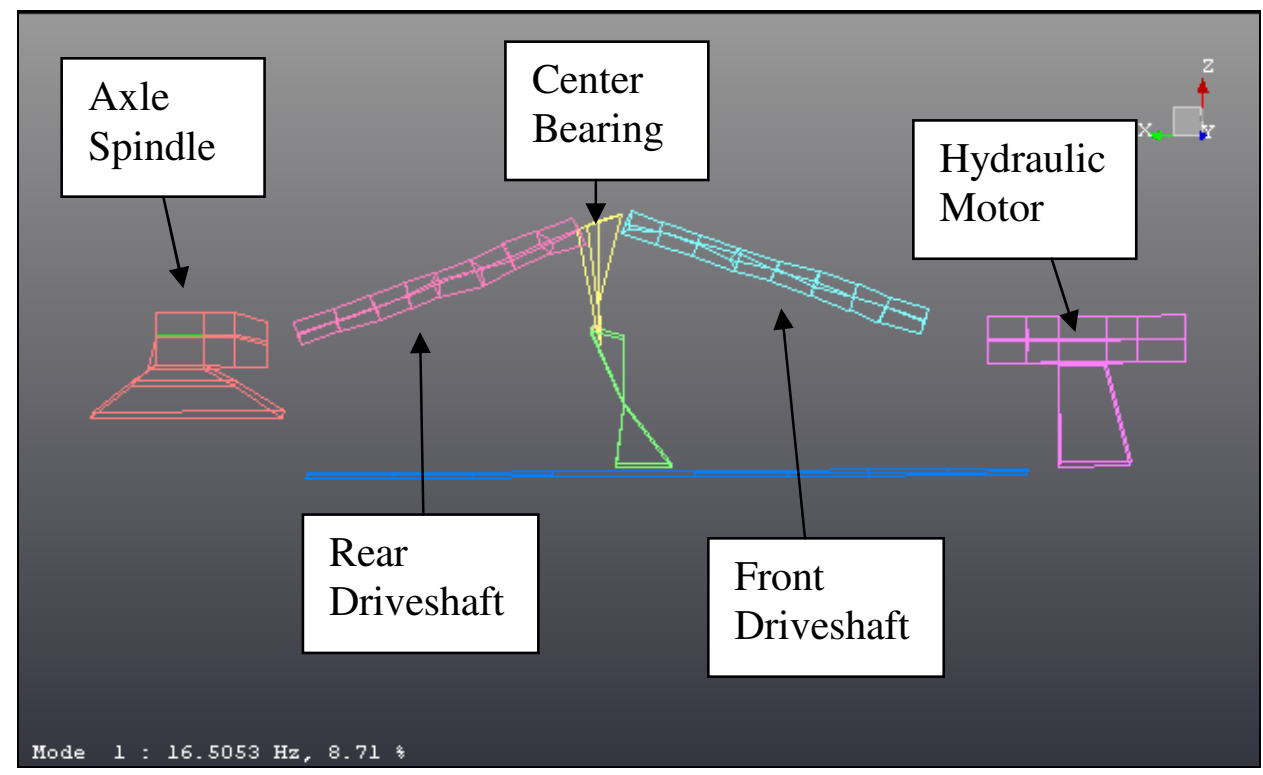

Fig. 19. Center Bearing Bounce Mode at 16.5 Hz. 
The center bearing bounce mode is basically the whole shaft system bouncing on the soft center bearing support. The shaft system articulates at the center universal joint on the front driveshaft side of the center bearing. The next two modes are pictured in Fig. 20.

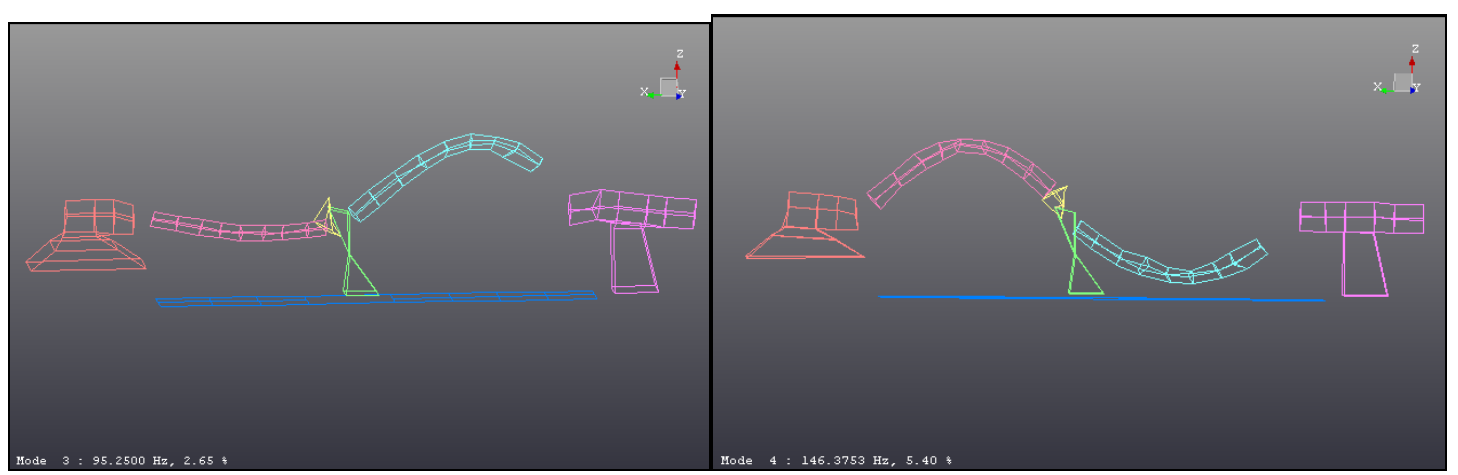

Fig. 20. Driveshaft Modes: a) Front Shaft Bending/ ITD Lower at $95 \mathrm{~Hz}$ and b) Front Driveshaft Bending/ ITD Upper at $146 \mathrm{~Hz}$.

The first two driveshaft bending modes both show the front driveshaft bending. The higher frequency mode has more movement with the rear driveshaft. Due to the inability to access the ITD for measurements, the mode shape descriptions were finalized after completing the analytical modal analysis revealing the influence of the ITD. The Front shaft bending/ITD lower has the front shaft and ITD moving out of phase with each other, thus providing enough inertial canceling to not require much movement of the rear shaft. The front shaft bending/ITD upper has the shaft and ITD moving in phase with each other, therefore moving more inertial which needs to be countered by inertial 
movement of the rear shaft. In addition, the proximity of the rear shaft bending mode influences the mode shape. The higher frequency bending modes are shown in Fig. 21.

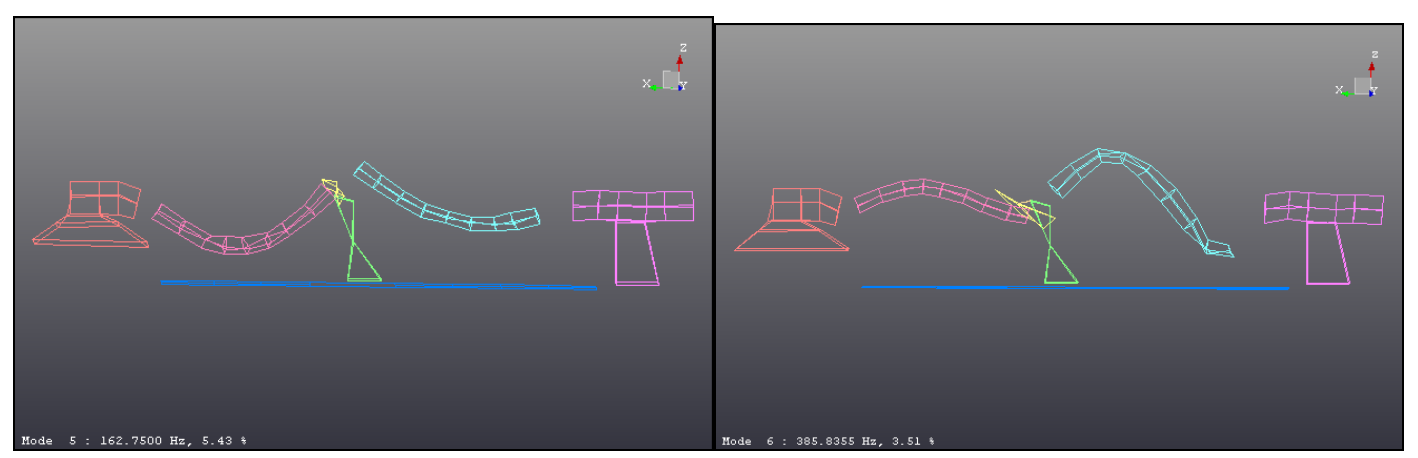

Fig. 21. Driveshaft Modes: a) Rear Shaft Bending at $162 \mathrm{~Hz}$ and b) Front Driveshaft Second Bending at $385 \mathrm{~Hz}$.

The rear shaft bending mode shows a significant bending of the rear shaft. Investigation of the phase of the FRF measurements confirms that the rear shaft is the active component. The front driveshaft is acting as inertia, but still shows some bending motion due to the proximity of frequency of the rear shaft bending mode and the front shaft bending/ITD upper mode. The highest mode at $385 \mathrm{~Hz}$ shows the front shaft bending with 2 antinodes, and therefore is termed the second bending.

The goal of the analytical model is to effectively replicate the dynamic response of the system, and therefore would need to produce relatively close natural frequencies and mode shapes. 


\section{Stiffness Characteristics}

Additional physical measurements were necessary to identify nonlinear characteristics of components other than the universal joint. Force vs. deflection and moment vs. deflection measurements were completed on all elastomeric components in the driveshaft system including the internal tuned damper and center bearing.

A driveshaft was modified with holes cut into the shaft to allow access to the ITD with a force and position transducers. The shaft was then clamped in a stiff fixture at each end of the ITD in the shaft. The fixture was clamped to a bedplate. A range of multiple forces was applied to the ITD and the corresponding displacement measured. A plot of the stiffness coefficient of the internal tuned damper is presented showing a cubic and a linear dependence of force on the deflection is presented in Fig. 22.

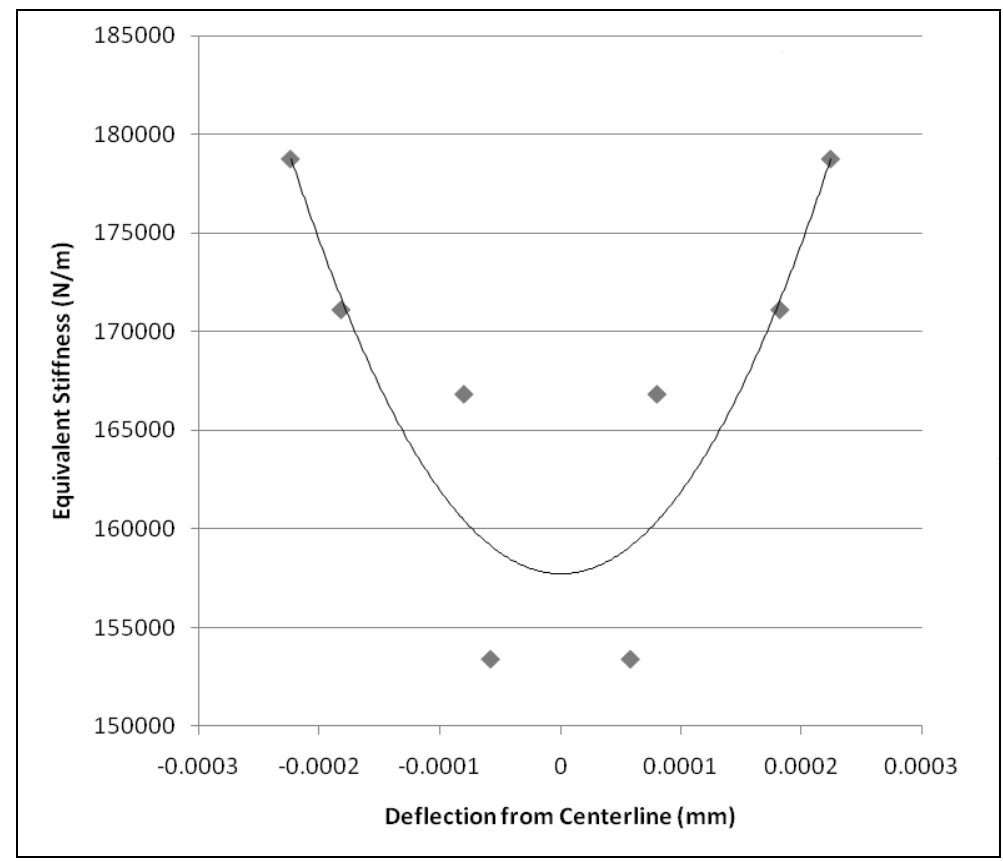

Fig. 22. Stiffness Measurements on ITD. 
Damping of the ITD was measured using a impact driving point FRF. The FRF integrated to $\mathrm{N} / \mathrm{m} / \mathrm{s}$ produced the damping term as estimate from the relatively flat line to the resonant frequency of the ITD. Therefore, from the measurements, the known mass, damping, and stiffness is incorporated into a mathematical SDOF system representing the ITD by:

$0.293 \ddot{x}+1.07 \dot{x}+1.577 * 10^{5} x+4.194 * 10^{11} x^{3}=0$

Where $\mathrm{x}$ is the relative displacement between the driveshaft and the ITD mass. This is the case in the range of deflections shown, however the dependence transitions from hardening to softening as the displacements increase significantly. A diagram of the ITD as installed in the driveshaft is pictured in Fig. 23.

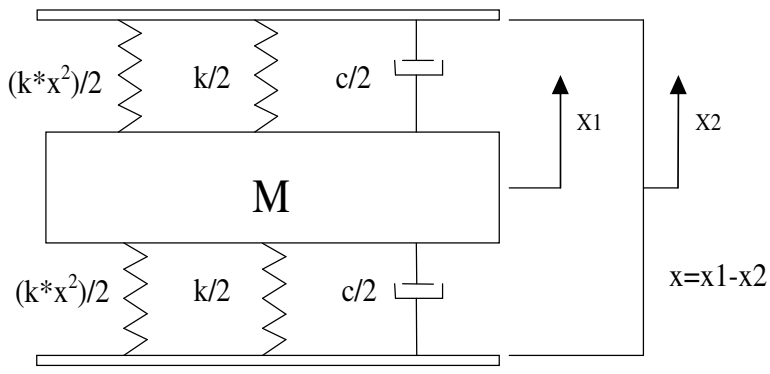

Fig. 23. Schematic of ITD in Driveshaft. 
Radial, axial and mesurements for the centerbearing reveal either a linear or a softening stiffness relationship with displacement. A moment vs. deflection mesurement of the centerbearing revealed nonlinear stiffness with a cubic and linear moment - deflection dependence as well, as depicted in Fig. 24.

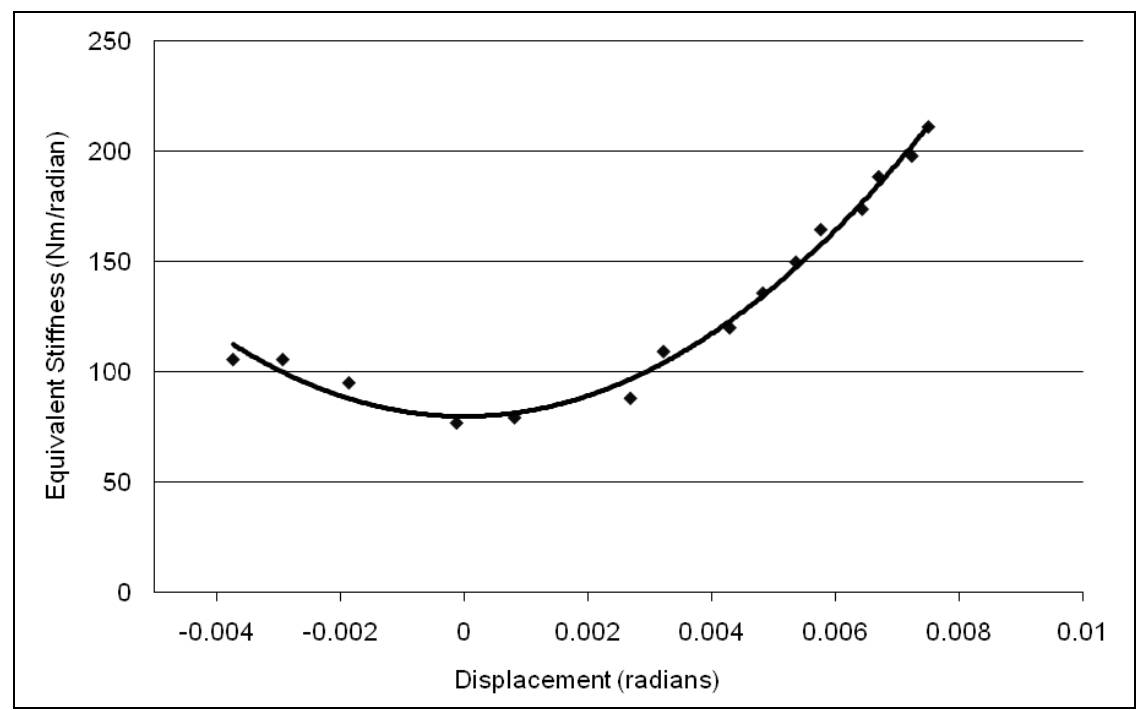

Fig. 24. Moment Stiffness Measurement of Driveshaft Center Bearing.

Therefore, due to multiple non-linearities in the system, it is difficult to identify the primary source of the nonlinearity with these measurements. Results from the model and subsequent analysis determined that the internal tuned damper was responsible for the jump. 


\section{Summary of Driveline System and Test Results}

The measured data provided significant evidence of a nonlinear jump in the runup portion of the operating test. The DOE provided insight into the significance of the misalignment angle of the center NCV joint. Of the tested misalignment factor levels, the highest misalignment level of 4 degrees was the only level to produce the jump. Imbalance was shown to have no significant influence on the nonlinear jump. The measured data showed good repeatability for all measurements. The jump comprised of the primary significant orders of $2^{\text {nd }}, 4^{\text {th }}$, and $8^{\text {th }}$ orders.

Modal Analysis revealed the key linear natural frequencies, resonances of the test system. This included the center bearing bounce mode and various shaft bending modes. It is interesting that only one of the primary 3 orders identified in the operating conditions is near a resonance near the jump. The $4^{\text {th }}$ order is at $150 \mathrm{~Hz}$, slightly higher than the front shaft bending/ITD upper mode shape identified. With the stiffening jump response, it is expected that the jump frequency would be slightly higher than the linear natural frequency that is active with the nonlinear jump. Analytical model correlation has the experimental modal results to replicate to insure a valid model.

The physical stiffness measurements revealed two possible sources of stiffening nonlinearities. These are in the elastomeric support of the ITD mass and the center bearing support. Physical stiffness and damping characteristics were determined for inclusion into the analytical system model. 


\section{CHAPTER III}

\section{DERIVATION OF EXCITATION MOMENT**}

\section{Non-constant Velocity Joint Characteristics}

The nature of a NCV joint turns the output shaft differently than the input shaft. The universal joint connects two shafts with a misalignment angle. The two axis of rotation intersect at the NCV joint and form a plane with the two shaft system. Each shaft creates a circular arc perpendicular to its axis of rotation as the system is rotated. Fig. 25 depicts these arcs as viewed from the axis of rotation of one of the shafts, and perpendicular to the shaft intersection plane.

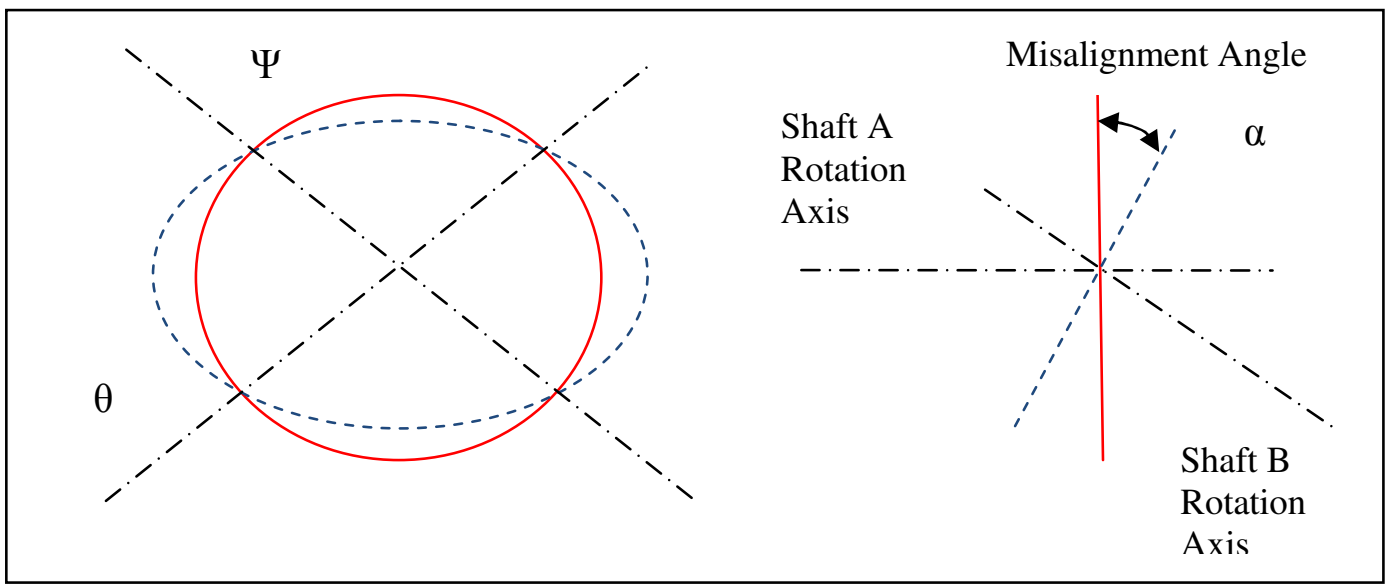

Fig. 25. Depictions of Shaft System Relative Rotations due to Misalignment.

*Part of this chapter is reprinted with permission from "Superharmonic nonlinear lateral vibrations of a segmented driveline incorporating a tuned damper excited by nonconstant velocity joints" by M. Browne and A. Palazzolo, 2009. Journal of Sound and Vibration, doi:10.1016/j.jsv.2008.12.018. 
The shaft A produced a perfect circle (red line) in this view, while the second shaft, $\mathrm{B}$, rotation produces an ellipse (blue dashed line). When shaft $\mathrm{A}$ is spun at a constant speed, the output shaft spins around the arc at this speed, and therefore creates a non-constant rotational speed on shaft B. Therefore, Non-constant Velocity (NCV) joint is an appropriate nomenclature.

From the geometry of the universal joint, the mathematical relationship of the position, velocity, and acceleration of the NCV joint have previously been documented[4] are

$$
\begin{aligned}
& \tan \Psi=\cos \alpha \tan \theta, \text { or } \quad \theta=\arctan \left(\frac{\tan (\psi)}{\cos (\alpha)}\right), \\
& \dot{\theta}=\frac{\omega \cos \alpha}{1-\sin ^{2} \Psi \sin ^{2} \alpha}, \quad \text { and } \\
& \ddot{\theta}=\frac{2 \dot{\theta}^{2} \cos \alpha \sin ^{2} \alpha \sin 2 \Psi}{\left(1-\sin ^{2} \alpha \sin ^{2} \Psi\right)^{2}} .
\end{aligned}
$$

The relationship between the input rotation angle, $\Psi$ and the output rotation angle, $\theta$ are dependent on the universal misalignment angle, $\alpha$. The velocity and acceleration terms clearly identify the non-constant nature of the movement of the output shaft relative to the input shaft moving at a constant speed. Wagner also developed simplified equations relating to the NCV joints using small angle approximations (less than 10 degrees) to highlight the primary second order excitation. The torsional vibrations for velocity and acceleration are

$$
\alpha_{1}=4 A \omega^{2} \sin 2 \beta, \quad \text { and }
$$




$$
\omega_{1}=\omega-2 A \omega \cos 2 \beta
$$

where $A=\operatorname{Arctan}\left(\frac{1}{\cos \theta}\right)^{\frac{1}{2}}-\operatorname{Arctan}(\cos \theta)^{\frac{1}{2}}$,

or simplified $\quad A \approx \frac{\theta^{2}}{2}$.

$\mathrm{NCV}$ joints not only produce torsional vibrations on the output shaft when the system is rotated with a misalignment angle across the joint, but the NCV joint also creates torsional torque oscillations from applied moments as the shaft system is rotated. Porat developed the moment equation for both the $\mathrm{CV}$ and $\mathrm{NCV}$ joint systems, this equation is

$$
M_{b}=M_{a} * \frac{\left(1-\frac{\cos \alpha}{T_{\phi}}\right)}{\sin \alpha}
$$

where $T_{\phi}$ is the speed ratio function,

$$
T_{\phi}=1
$$

for a $\mathrm{CV}$ joint and

$$
T_{\phi}=\frac{\left(1-\sin ^{2} \alpha * \cos ^{2} \Psi\right)}{\cos \alpha}
$$

for a NCV joint.

Porat's derivation assumed only the average static load distribution, inflexible shafts, and inertia loading was ignored. Furthering the moment derivation for excitation into shaft systems, Ota developed a moment equation for a secondary moment, acting perpendicular to the shaft axis of rotation $[20,21]$. This secondary moment is defined by 


$$
M_{\theta}^{\prime \prime}=M_{\theta} * \sin \alpha^{*} \frac{\cos \Psi}{\sqrt{1-\sin ^{3} \alpha^{*} \cos ^{2} \Psi}} .
$$

where $M_{\theta}^{\prime \prime}$ is the secondary moment, $M_{\theta}$ is the load moment on shaft $\mathrm{B}, \alpha$ and $\Psi$ are the misalignment and input shaft rotation angles. This shows a dependence on the applied load at the end of the shaft system. Inertia or no applied moment to the shaft system was considered.

Due to the lack of inclusion of inertial loading, neither of these moment equations is adequate for application to the driveshaft system presented. Therefore, a derivation, based on Ota's derivation was necessary to determine the secondary moment with no applied load. This will provide an appropriate excitation moment into the shaft system for subsequent analysis.

\section{Derivation of the Secondary Moment}

As outlined in reference paper [21], $\mathrm{p}$ and $\mathrm{q}$ are the orthogonal vectors of the cross pin as pictured in Fig. 26. The vectors change as a function of the input rotation angle, $\Psi$, or the output rotation angle, $\theta$. These vectors are also dependent on the universal misalignment angle, $\alpha$. 


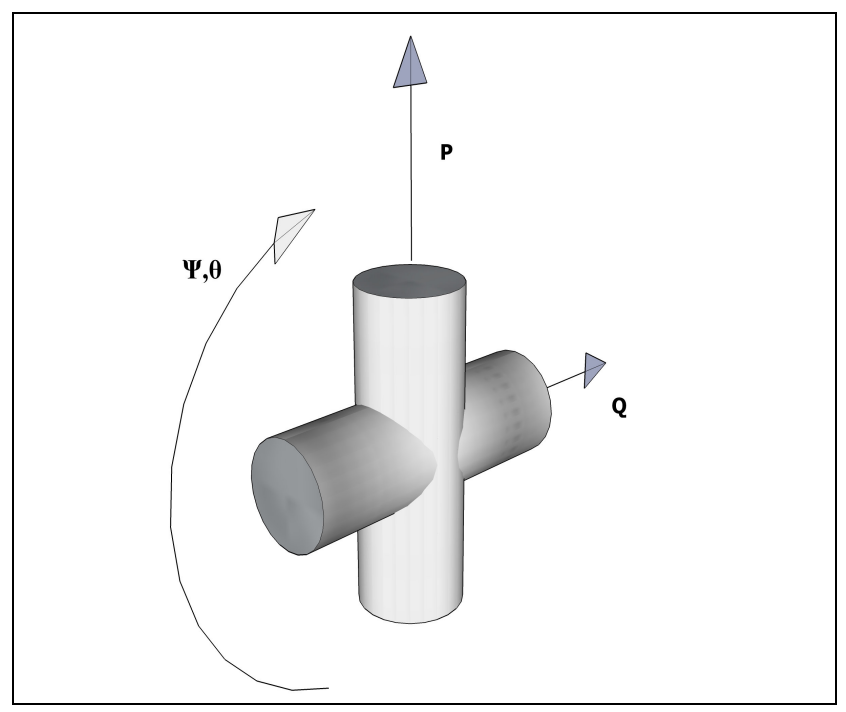

Fig. 26. Universal Joint Coordinate System.

The global coordinate system is defined as having the $\mathrm{z}$-direction along the axis of rotation of the output shaft, $\mathrm{Y}$ in the vertical, and $\mathrm{X}$ in the axis parallel to the ground into the plot. This coordinate system's orientation is pictured in Figure 27. In addition, the three angles: $\psi, \alpha$, and $\theta$ are shown for the following derivation.

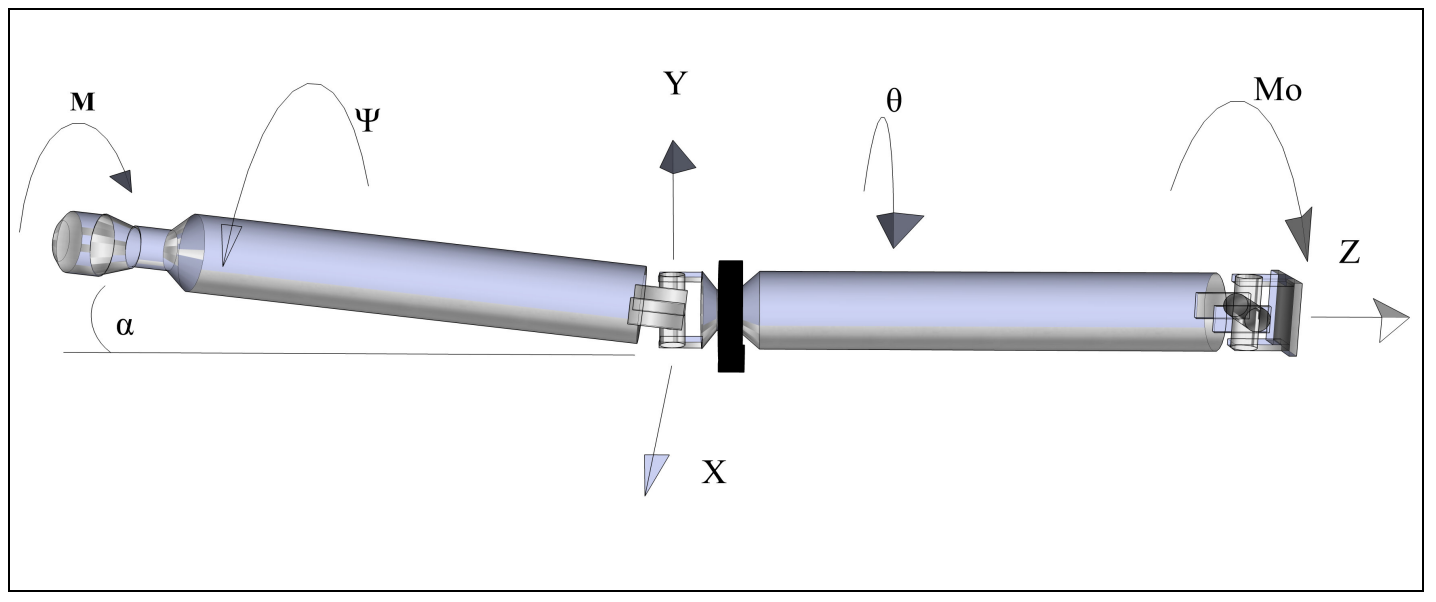

Fig. 27. Driveshaft Coordinate System. 
Due to the nature of the universal joint, the only moment acting on the cross pin is perpendicular to the cross pin, assuming frictionless interaction between the cross pin and the shaft yokes. This direction is mathematically defined by the cross product of vectors $\mathrm{p}$ and $\mathrm{q}$. Assuming the magnitude of the input moment is $M$, then the moment on the cross pin is derived as

$$
\begin{aligned}
& \mathbf{M}=-M^{*} \sin (\alpha) * \cos (\psi) * \cos (\theta) \mathbf{i}-M^{*} \sin (\alpha) * \cos (\psi) \\
& * \sin (\theta) \mathbf{j}+M^{*}(\cos (\alpha) * \cos *(\psi) * \cos (\theta)+\sin (\psi) * \sin (\theta)) \mathbf{k}
\end{aligned}
$$

Due to the complexity of the joint, $M$ is not known or typically measured. The output shaft moment, $M o$, is known. Therefore, $M$ can be calculated from $M o$ as

$$
M o=M *(\cos (\alpha) * \cos *(\psi) * \cos (\theta)+\sin (\psi) * \sin (\theta)) .
$$

The output shaft only has reaction moments in the axial, $\mathrm{z}$ direction, due to the transmission of torque or angular acceleration of the output shaft assuming no friction between the cross and the shaft yokes. Solving for $M$ and substituting it into Eq. (16) yields the vector form of the moment on the cross pin as

$$
\begin{aligned}
& \mathbf{M}=-\frac{\sin (\alpha) * \cos (\psi) * \cos (\theta) * M o}{(\cos (\alpha) * \cos *(\psi) * \cos (\theta)+\sin (\psi) * \sin (\theta))} \mathbf{i} \\
& -\frac{\sin (\alpha) * \cos (\psi) * \sin (\theta) * M o}{\left(\cos (\alpha) * \cos ^{*}(\psi) * \cos (\theta)+\sin (\psi) * \sin (\theta)\right)} \mathbf{j}+(M o) \mathbf{k}
\end{aligned} .
$$

Finally, the secondary moment, $M s$, is defined by a moment perpendicular to the output moment, $M o$, and also perpendicular to the direction of the cross pin connected to 
the rear shaft yoke direction, q. The magnitude of the secondary moment and its vector is defined as

$$
\begin{aligned}
& M s=-\frac{\sin (\alpha) * \cos (\psi) * M o}{(\cos (\alpha) * \cos *(\psi) * \cos (\theta)+\sin (\psi) * \sin (\theta))} \quad \text { and } \\
& \mathbf{M s}=-\frac{\sin (\alpha) * \cos (\psi)^{2} * \cos (\alpha) * M o}{\left(1-\sin (\alpha)^{2} * \cos (\psi)^{2}\right)} \mathbf{i}-\frac{\sin (\alpha) * \cos (\psi) * \sin (\psi) * M o}{\left(1-\sin (\alpha)^{2} * \cos (\psi)^{2}\right)} \mathbf{j}
\end{aligned}
$$

in the global coordinate system. The resultant output moment, $M o$, is comprised of all moments applied to the rear shaft defined by

$$
\begin{aligned}
& \sum M=I * \ddot{\theta}, \\
& M o+M d=I * \ddot{\theta}, \text { and } \\
& M o=I * \ddot{\theta}-M d .
\end{aligned}
$$

In this case, the total moments are the output moment and a resultant driving torque at the axle end of the driveshaft, $M d$. This assumes the rear shaft is rigid in the torsional direction, thereby having no potential or stored energy. The input angle can be defined as a function of time and the rotational speed, $\omega$, in

$$
\psi(t)=\omega^{*} t
$$


Substituting into Eq. (2) and derivating once and twice produces

$$
\begin{aligned}
& \theta(t)=\arctan \left(\frac{\tan \left(\omega^{*} t\right)}{\cos (\alpha)}\right), \\
& \frac{d}{d t} \theta(t)=\frac{\omega^{*} \cos (\alpha)}{\left(1-\sin (\alpha)^{2} * \cos \left(\omega^{*} t\right)^{2}\right)}, \text { and } \\
& \frac{d^{2}}{d t^{2}} \theta(t)=-\frac{2 * \omega^{2} * \cos (\alpha)^{*} \sin (\alpha)^{2} * \cos \left(\omega^{*} t\right)^{*} \sin \left(\omega^{*} t\right)}{\left(1-\sin (\alpha)^{2} * \cos \left(\omega^{*} t\right)^{2}\right)^{2}}
\end{aligned}
$$

Substituting the acceleration term, Eq. (25) into Eq. (21) results in

$$
M o=-\frac{2 * I * \omega^{2} * \cos (\alpha) * \sin (\alpha)^{2} * \cos \left(\omega^{*} t\right)^{*} \sin \left(\omega^{*} t\right)}{\left(1-\sin (\alpha)^{2} * \cos \left(\omega^{*} t\right)^{2}\right)^{2}}-M d
$$

Furthermore, Eq. (26) can now be substituted into Eq. (20) to obtain the secondary moment. The secondary moments in the $\mathrm{x}$ and $\mathrm{y}$ direction are

$$
\begin{gathered}
M s_{x}=\left(\begin{array}{l}
-\frac{2 * I * \sin (\alpha)^{3} * \cos \left(\omega^{*} t\right)^{3} * \cos (\alpha)^{2} * \omega^{2} * \sin \left(\omega^{*} t\right)}{\left(-1+\sin (\alpha)^{2} * \cos \left(\omega^{*} t\right)^{2}\right)^{3}} \\
-\frac{\sin (\alpha)^{*} \cos \left(\omega^{*} t\right)^{2} * \cos (\alpha)^{*} M d}{\left(-1+\sin (\alpha)^{2} \cos \left(\omega^{*} t\right)^{2}\right)}
\end{array}\right), \text { and } \\
M s_{y}=\left(\begin{array}{l}
-\frac{2 * I * \sin (\alpha)^{3} \sin \left(\omega^{*} t\right)^{2} * \cos \left(\omega^{*} t\right)^{2} * \omega^{2} * \cos (\alpha)}{\left(-1+\sin (\alpha)^{2} * \cos \left(\omega^{*} t\right)^{2}\right)^{3}} \\
-\frac{\sin (\alpha)^{*} \sin \left(\omega^{*} t\right)^{*} \cos \left(\omega^{*} t\right)^{*} M d}{\left(-1+\sin (\alpha)^{2} \cos \left(\omega^{*} t\right)^{2}\right)}
\end{array}\right) .
\end{gathered}
$$

The moments in the $\mathrm{x}$ and $\mathrm{y}$ directions are not equal. This is due to the misalignment was completed in one plane, but the secondary moment is not just in the 
plane of misalignment and in fact changes direction as the joint rotates thereby not aligning with either plane. This is an effect stemming from Eq. (16). Therefore with effects of the moment will influence the moments in both planes differently.

Due to the equations complexity, it is unclear as to the frequency or order content of Eqs. (27-28). For further analysis, it needs to be shown that the Fourier series expansion of the secondary moment also has multiple excitation orders, or harmonics of the known second order excitation due to the universal joint. The following analysis derives the, Fourier expansion for the secondary moment. The self-excitation of the universal joint is presented in the first part of the equation of both moment directions. A misalignment angle of 4 degrees is used to simplify the equation, and is the primary angle of focus of the system's response from the physical test results. The dynamic secondary moment is presented subtracting any input load on the driveshaft, resulting in $M s_{x}=\left(-\frac{2 * I * \sin \left(\frac{\pi}{45}\right)^{3} * \cos (\psi)^{3} * \cos \left(\frac{\pi}{45}\right)^{2} * \omega^{2} * \sin (\psi)}{\left(-1+\sin \left(\frac{\pi}{45}\right)^{2} * \cos (\psi)^{2}\right)^{3}}\right)$.

Subsequently, the Fourier series for a 4 degree angle is

$$
\begin{aligned}
& M s_{x}=0.00017032 * I * \omega^{2} * \sin (2 * \psi)+0.000085770 * I * \omega^{2} * \sin (4 * \psi) \\
& +4.2873 * 10^{-7} * I * \omega^{2} * \sin (6 * \psi)+6.6040 * 10^{-8} I * \omega^{2} * \sin (8 * \psi)
\end{aligned} .
$$

Fig. 28 presents the quality of the fit of the Fourier series with respect to the original equation. The Fourier series with the first two terms was reasonably close to the four term approximation. This is also verified by comparing the coefficients of the various terms, as the first two terms are significantly larger than that of the higher order 
terms. For demonstration purpose only, the values of $\omega=1$, and $I=1000$ were used to generate Fig. 28.

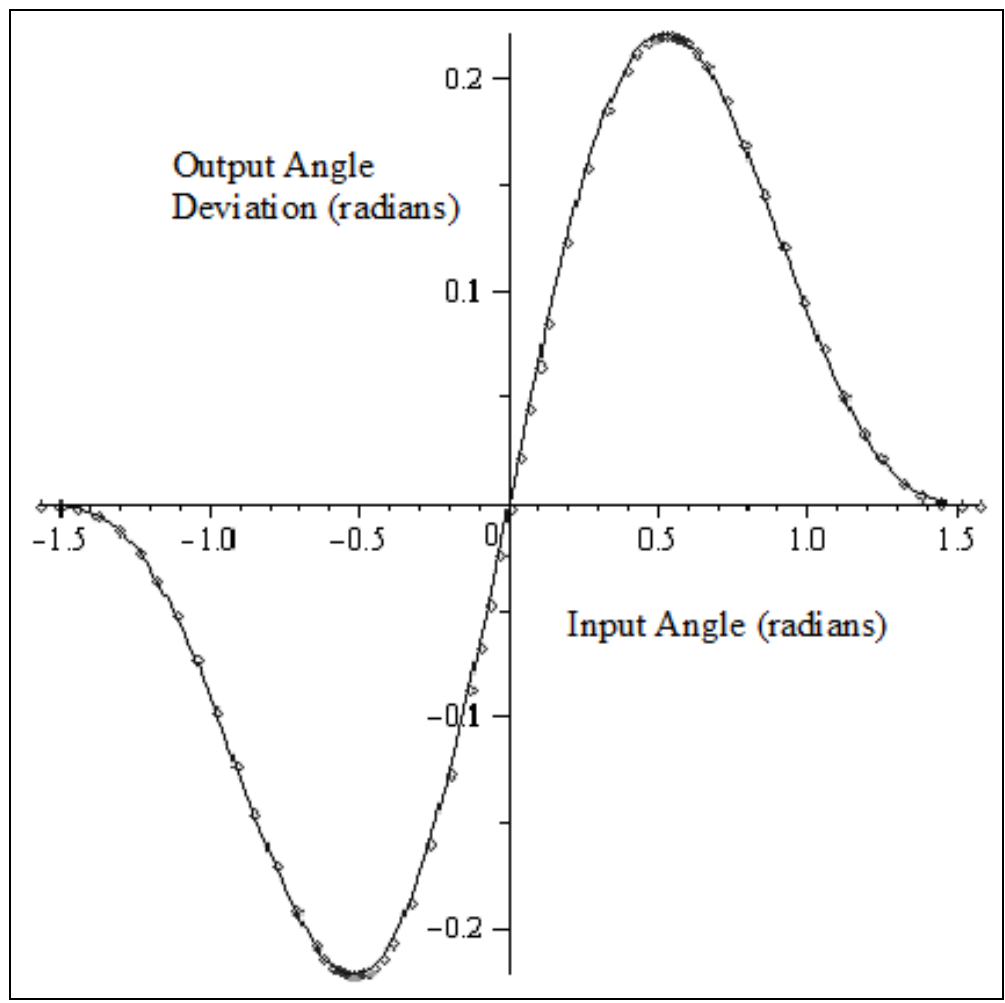

Fig. 28. Comparison of Full Moment and Fourier Series Approximation.

Therefore, it is clear that the Fourier series approximation of the x-direction self excitation comprises also of the primary even integer multiples previously presented in the angular deflection and acceleration. The y-direction derivation of the self-excitation term in Eq. (28) calculated without the load torque is 


$$
M s_{y}=\left(-\frac{2 * I * \sin \left(\frac{\pi}{45}\right)^{3} * \sin (\psi)^{2} * \cos (\psi)^{2} * \omega^{2} * \cos \left(\frac{\pi}{45}\right)}{\left(-1+\sin \left(\frac{\pi}{45}\right)^{2} * \cos (\psi)^{2}\right)^{3}}\right) .
$$

Subsequently, the Fourier series is

$$
\begin{aligned}
& M s_{y}=0.000085258 * I * \omega^{2}+2.8582 * 10^{-7} * I * \omega^{2} * \cos (2 * \psi) \\
& -0.000085265 * I * \omega^{2} * \cos (4 * \psi)-3.6917 * 10^{-7} * I * \omega^{2} * \cos (6 * \psi) . \\
& +8.357010 * 10^{-7} I * \omega^{2} * \cos (8 * \psi)
\end{aligned}
$$

Fig. 29 presents the quality of the fit of the Fourier series with respect to the original equation. Once again, the Fourier series with the first two terms was reasonably close to the four term approximation. The coefficients indicate that the fourth order term dominates, as the coefficient is significantly larger than those of the other terms. For demonstration purpose only, the values of $\omega=1$, and $I=1000$ were used to generate the results plotted in Fig. 29. 


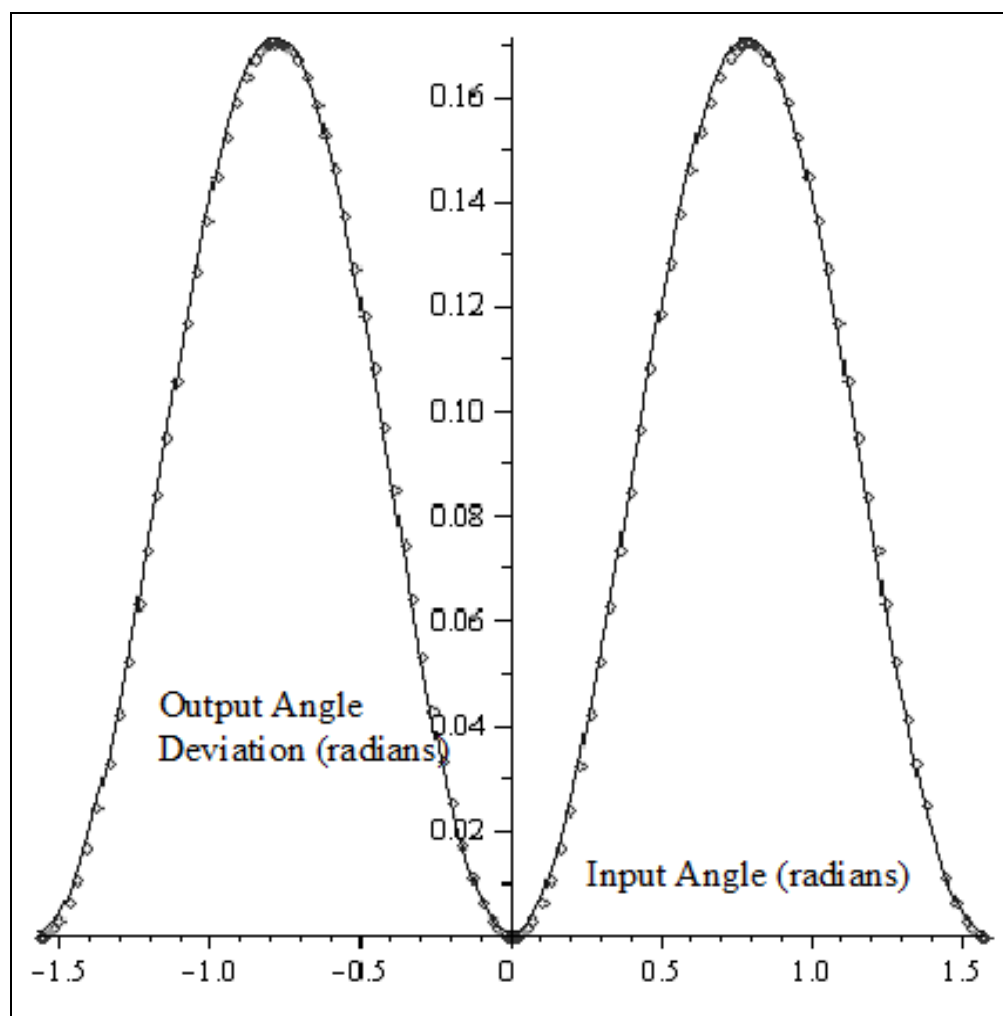

Fig. 29. Comparison of Full Moment and Fourier Series Approximation of Secondary Moment.

This moment is comprised of significant harmonic excitation, especially the $2^{\text {nd }}$ order in the $\mathrm{x}$-direction, and $4^{\text {th }}$ order in the y-direction.

\section{Summary of Secondary Moment Characteristics}

The derived secondary moment has a few unique characteristics. First the moment is a resultant of either or both an applied load or inertial load to the shaft system. The equation of this applied moment was necessary to be derived, and mot included in previous research. 
Second, the secondary moment is comprised of two components. One component acts in the plane of the shaft system, perpendicular to the shaft. The other component acts perpendicular to the plane created by the intersection of the shaft axes. The two components are not equivalent. This is due to the direction of the calculated secondary moments as oriented with respect to the angle of misalignment.

Third, the secondary moments produce excitations at even multiples of the shaft rotation. One secondary moment produces the expected high level of $2^{\text {nd }}$ order excitations with decreasing excitation amplitude with increased even multiple orders.

The other of the secondary moments produces a static moment as well as higher amplitude excitations from the $4^{\text {th }}, 6^{\text {th }}$, and $8^{\text {th }}$ orders than the expected amplitude of the $2^{\text {nd }}$ order.

The derivation of the secondary moment provides four possible equations for calculating responses. The full derived force equation will provide all excitation frequencies into the system. Use of the Fourier series equations provides the ability to select specific excitation orders into the shaft system. This will help isolate the jump phenomena to what order and natural frequency creates the jump phenomena. The two secondary moments will also help isolate witch direction contribution is more important in this systems' response. 


\section{CHAPTER IV}

\section{ANALYTICAL MODEL OF DRIVELINE SYSTEM*}

\section{Derivation of Beam Model}

Due to the complexity of the driveshaft system, significant work was completed in deriving a system of equations representing the physical system. This work was previously presented in a publication outlining the key results[43]. This summary provides more detail and subsequent work from the previous publication.

A lumped mass beam element model was chosen for the FEA analysis. This lumped beam model has previously been used with good correlation results for previous work on similar driveshaft analysis. The lumped mass helps to simplify the FEA model by only having diagonal terms for the mass matrix. Therefore, the FEA model consists of massless beam elements and concentrated inertias as shown in Fig. 30.

*Part of this chapter is reprinted with permission from "Superharmonic nonlinear lateral vibrations of a segmented driveline incorporating a tuned damper excited by nonconstant velocity joints." by M. Browne and A. Palazzolo, 2009. Journal of Sound and Vibration, doi:10.1016/j.jsv.2008.12.018. 


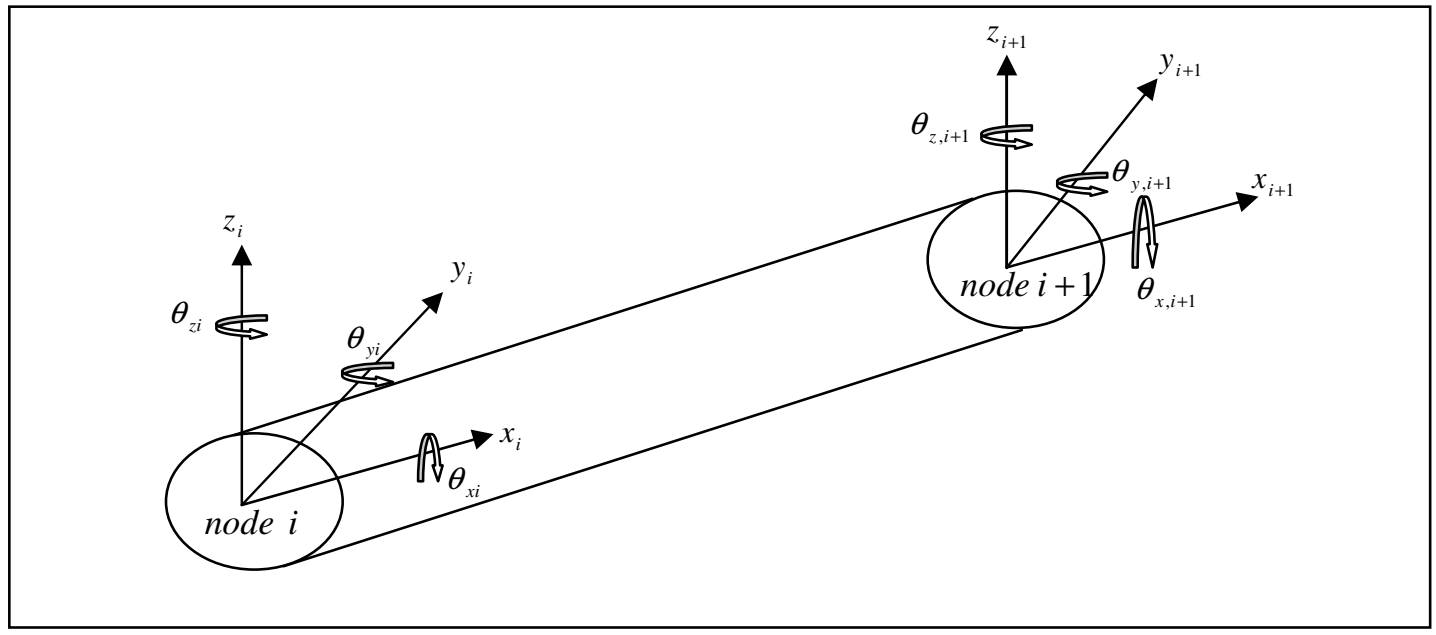

Fig. 30. Beam Element Coordinate System Definition.

The nodal rotational and translational degrees of freedom of the 2-noded, 6 degree of freedom per node beam element in Fig 30 are arranged in the element displacement vector with the following convention of

$$
U_{e}=\left[\begin{array}{lllllllllllll}
x_{i} & y_{i} & z_{i} & \theta_{x i} & \theta_{y i} & \theta_{z i} & x_{i+1} & y_{i+1} & z_{i+1} & \theta_{x, i+1} & \theta_{y, i+1} & \theta_{z, i+1}
\end{array}\right]^{T} .
$$

Subsequently, the diagonal lumped mass matrix and stiffness matrix for the beam element are

$$
M_{e}=\operatorname{diag}\left(\left[\begin{array}{lllllllllllll}
m_{i} & m_{i} & m_{i} & I_{p, i} & I_{t, i} & I_{t, i} & m_{i+1} & m_{i+1} & m_{i+1} & I_{p, i+1} & I_{t, i+1} & I_{t, i+1}
\end{array}\right]\right) \text { and }
$$




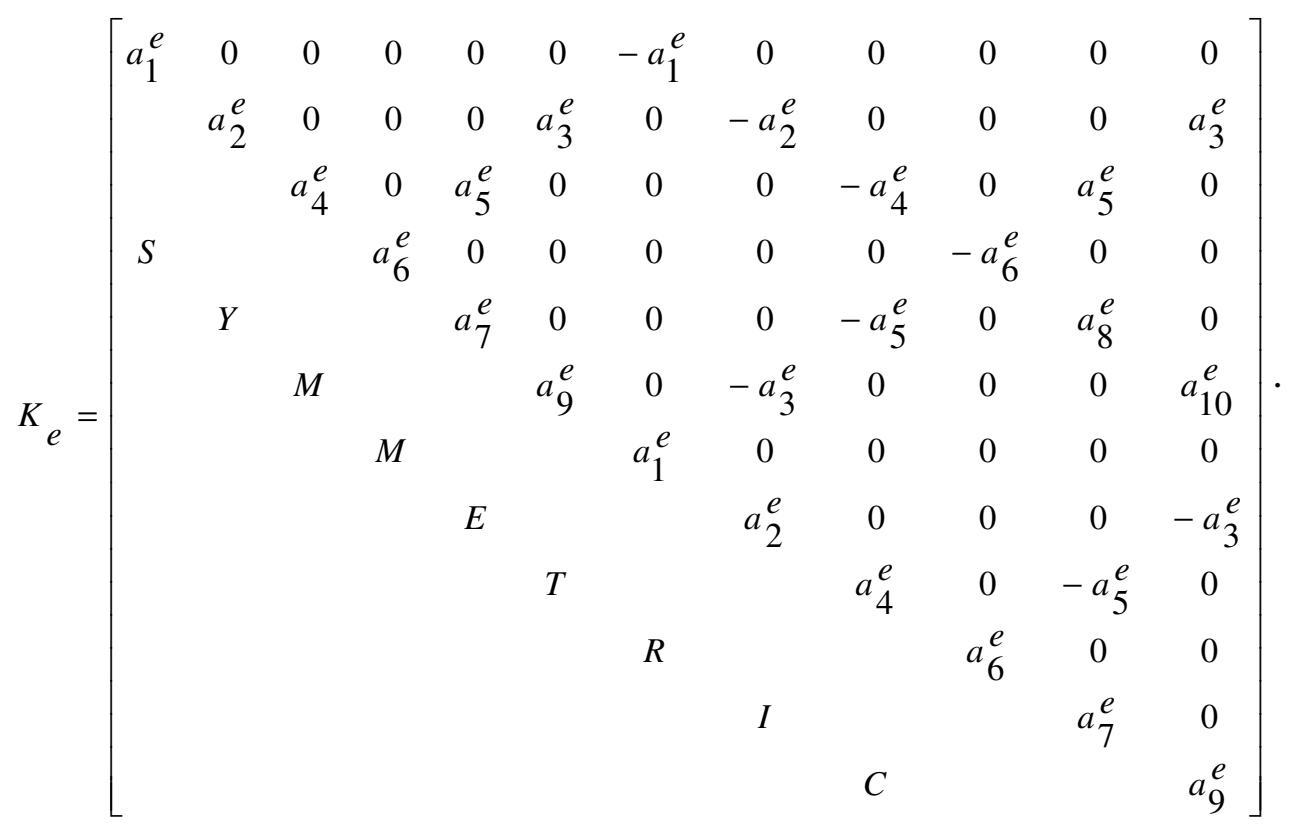

where the coefficients are defined as

$$
\begin{aligned}
& a_{1}^{e}=E^{e} A^{e} / L_{e}, \quad a_{2}^{e}=12 E^{e} I_{x 3}^{e} / L_{e}^{3}, \quad a_{3}^{e}=6 E^{e} I_{x 3}^{e} / L_{e}^{2}, \quad a_{4}^{e}=12 E^{e} I_{x 2}^{e} / L_{e}^{3}, \\
& a_{5}^{e}=-6 E^{e} I_{x 2}^{e} / L_{e}^{2}, \quad a_{6}^{e}=G^{e} J^{e} / L_{e}, \quad a_{7}^{e}=4 E^{e} I_{x 2}^{e} / L_{e}, \\
& a_{8}^{e}=2 E^{e} I_{x 2}^{e} / L_{e}, a_{9}^{e}=4 E^{e} I_{x 3}^{e} / L_{e}, \text { and } a_{10}^{e}=2 E^{e} I_{x 3}^{e} / L_{e} .
\end{aligned}
$$

Initial finite element modeling of the driveshaft with beam elements spaced along the tube, and at every major change of inner or outer diameters produces a system with 162 degrees of freedom. This model was very large and thereby needed to be significantly reduced. After a few iterations of model development, a 20 degree of freedom system was developed. This system of equations was not sufficient to produce decent simulation results. This is due to the required small time step to get results from the system with the highest natural frequency of $44 \mathrm{kHz}$. Therefore development to 
simplify the system of equations to a level acceptable for simulation results was necessary.

\section{Modal Truncation}

The development of the final simplified equations of motion included many steps starting with a small FE model of the separate front and rear driveshafts independently. Node locations for the driveshafts are presented in Fig. 31. It is important to note that node locations 7 and 8 are coincident when assembled.

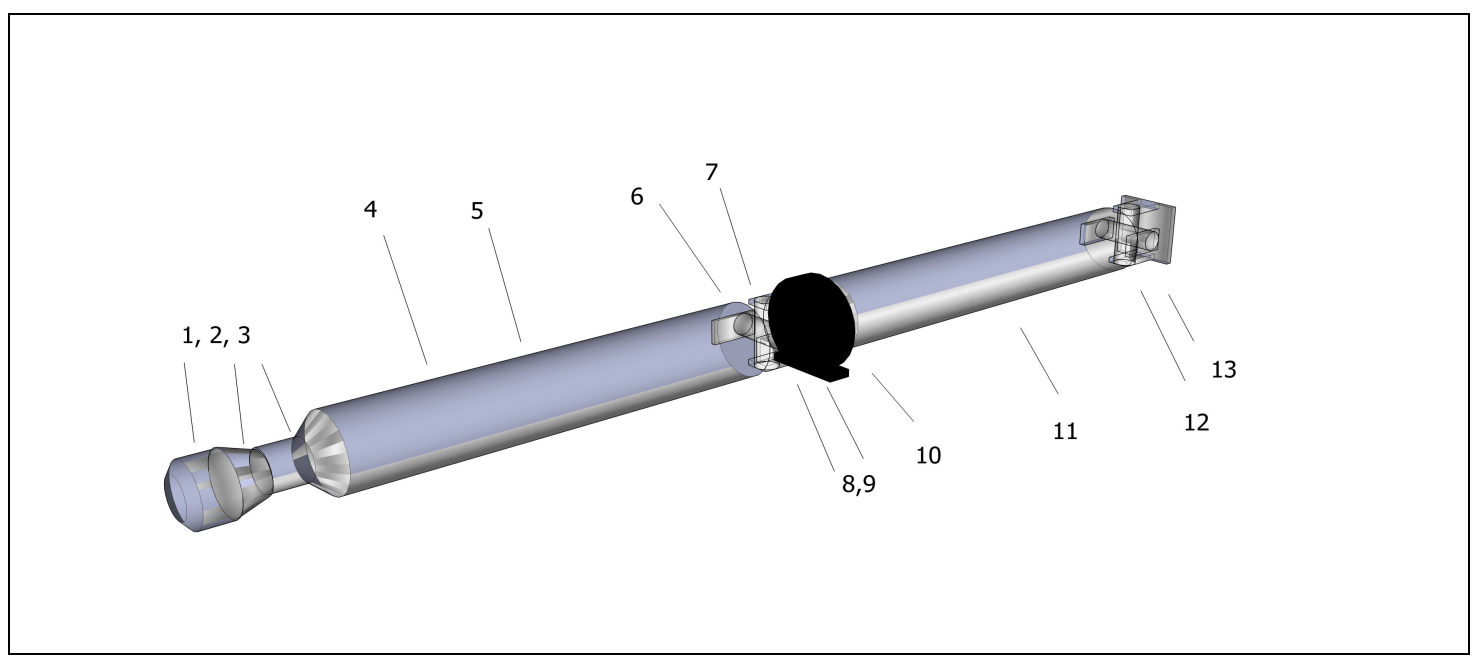

Fig. 31. Node Locations of Final Model.

Modal equations of motion were calculated using standard analytical modal analysis techniques to determine natural frequencies and mode shapes of each of the front and rear driveshafts. The front shaft was constrained only with a pin joint to 
ground at node 1 . The resultant natural frequencies less than $500 \mathrm{~Hz}$ are presented in

Table 2.

Table 2

Front Driveshaft Natural Frequencies

Mode Normal Modes

Rigid Body Rotation $\quad 0$

Rigid Body Rotation $\quad 0$

Front Driveshaft Bending 147

Front Driveshaft Bending $\quad 456$

The two rigid body modes are necessary for subsequent assembly in the modal domain. The rear driveshaft was supported with a pin joint connected to ground at node 13 similar to driveshaft 1 . A linear spring support was placed between ground and point 10 acting as the linear portion of the center bearing support. The spring stiffness added was that measured previously on the physical center bearing radial stiffness. Table 3 presents the rear driveshaft natural frequencies under $500 \mathrm{~Hz}$.

Table 3

Rear Driveshaft Natural Frequencies Mode Normal Modes

Center Bearing Bounce 15.6

Rear Driveshaft Bending 
The results from the modal analysis on the rear driveshaft provide 2 significant modes of the bounce mode on the center bearing, and the first bending mode of the rear driveshaft. From the modal analysis, in addition to the presented eigenvalues, eigenvectors for each mode were calculated. From these eigenvectors, the modal mass and stiffness can then be calculated.

These modal equations of motions were truncated to use only the equations with the natural frequencies in the range of study, modes with natural frequencies less than $500 \mathrm{~Hz}$ previously presented. This included the calculated modal mass and stiffness. Modal damping of the modes was added via modal damping measured calculated from the experimental modal analysis for each correlated mode. The forcing function was added to the universal joint location and transformed to the modal domain using the eigenvectors. The resultant 6 DOF system of equations with 2 separated sets of modal equations and DOFs are 


$$
\begin{aligned}
& {\left[\begin{array}{cccc:cc}
m_{f 1} & 0 & 0 & 0 & 0 & 0 \\
0 & m_{f 2} & 0 & 0 & 0 & 0 \\
0 & 0 & m_{f 3} & 0 & 0 & 0 \\
0 & 0 & 0 & m_{f 4} & 0 & 0 \\
\hdashline 0 & 0 & 0 & 0 & m_{r 1} & 0 \\
0 & 0 & 0 & 0 & 0 & m_{r 2}
\end{array}\right]\left\{\begin{array}{l}
\ddot{q}_{f 1} \\
\ddot{q}_{f 2} \\
\ddot{q}_{f 3} \\
\ddot{q}_{f 4} \\
\hdashline \ddot{q}_{r 1} \\
\ddot{q}_{r 2}
\end{array}\right\}+} \\
& {\left[\begin{array}{cccc:cc}
2 * \zeta_{f 1} * \omega_{n f 1} & 0 & 0 & 0 & 0 & 0 \\
0 & 2 * \zeta_{f 2} * \omega_{n f 2} & 0 & 0 & 0 & 0 \\
0 & 0 & 2 * \zeta_{f 3} * \omega_{n f 3} & 0 & 0 & 0 \\
0 & 0 & 0 & 2 * \zeta_{f 4} * \omega_{n f 4} & 0 & 0 \\
\hdashline 0 & 0 & 0 & 0 & 2 * \zeta_{r 1} * \omega_{n r 1} & 0 \\
0 & 0 & 0 & 0 & 0 & 2 * \zeta_{r 2} * \omega_{n r 2}
\end{array}\right]\left[\begin{array}{l}
\dot{q}_{f 1} \\
\dot{q}_{f 2} \\
\dot{q}_{f 3} \\
\dot{q}_{f 4} \\
\dot{q}_{r 1} \\
\dot{q}_{r 2}
\end{array}\right\}+} \\
& {\left[\begin{array}{cccc:cc}
k_{f 1} & 0 & 0 & 0 & 0 & 0 \\
0 & k_{f 2} & 0 & 0 & 0 & 0 \\
0 & 0 & k_{f 3} & 0 & 0 & 0 \\
0 & 0 & 0 & k_{f 4} & 0 & 0 \\
\hdashline 0 & 0 & 0 & 0 & k_{r 1} & 0 \\
0 & 0 & 0 & 0 & 0 & k_{r 2}
\end{array}\right]\left\{\begin{array}{c}
q_{f 1} \\
q_{f 2} \\
q_{f 3} \\
q_{f 4} \\
\hdashline q_{r 1} \\
q_{r 2}
\end{array}\right\}=\left\{\begin{array}{l}
\phi_{f 1}(7) \\
\phi_{f 2}(7) \\
\phi_{f 3}(7) \\
\phi_{f 4}(7) \\
\hdashline \phi_{r 1}(8) \\
\phi_{r 2}(8)
\end{array}\right\} * f(t, \omega, \alpha) .}
\end{aligned}
$$

where subscripts $f 1-4$ are attributed to the front driveshaft model mode shapes $1-4, r 1-2$ are attributed to the rear driveshaft model mode shapes 1-2. The modal masses $(m)$ stiffnesses $(k)$, damping ratios $(\zeta)$, natural frequencies $\left(\omega_{n}\right)$ and coordinates $(q)$ are key characteristics from each driveshaft's modal equations of motion. The applied forcing function of the secondary moment, $\mathrm{f}(\mathrm{t}, \omega, \alpha)$ is transformed to the modal domain by multiplication of the eigenvector $(\varphi)$ of the respective modes at the position of the universal joint.

These sets of equations give the linear basis for the system including all the modes in the frequency range of interest. Subsequently, as the entire equation is linear, 
and the inclusion of the ITD has not been considered, additional changes and additions to the system of equations are necessary.

\section{Modal Modification}

The internal tuned damper was added to the front driveshaft using an additional degree of freedom coupled to the front driveshaft modal equations of motion at the node where it is located in the driveshaft. This attachment is completed using the mode shape scaling functions necessary for physical to modal or modal to physical transformations. The basic physical domain equation of motion for the addition of the ITD is

$m_{I T D} \ddot{x}_{I T D}+c_{I T D}\left(\dot{x}_{I T D}-\dot{x}_{5}\right)+k\left(x_{I T D}-x_{5}\right)+k c u b e\left(x_{I T D}-x_{5}\right)^{3}=0$

where subscripts of ITD reference the ITD characteristics of position $(x)$, mass $(m)$, damping $(c)$, and stiffness $(k)$. The position that the damper is located and the stiffness and damping is connected to is represented by $x_{5}$. The ITD can remain as a physical coordinate as it is an added DOF, but the position of the shaft needs to be transformed to the modal coordinate system to allow for integration into the modal system of equations. This transformation is

$x_{5}=\phi_{f 1}(5) * q_{f 1}+\phi_{f 2}(5) * q_{f 2}+\phi_{f 3}(5) * q_{f 3}+\phi_{f 4}(5) * q_{f 4}$

where the $\varphi$ and $q$ are the eigenvectors and modal coordinates of the front shaft modes previously noted. Eq. (35) can now be substituted into Eq. (34) to obtain the necessary 
equation of the ITD. Subsequent reciprocal stiffness and damping is also added to the modal equations of motion to complete the addition of the ITD. A similar approach is used to add the center bearing nonlinear moment stiffness to the equation. The set of modal equations of motion now becomes

$$
\begin{aligned}
& {\left[\begin{array}{cccc:cc|c}
m_{f 1} & 0 & 0 & 0 & 0 & 0 & 0 \\
0 & m_{f 2} & 0 & 0 & 0 & 0 & 0 \\
0 & 0 & m_{f 3} & 0 & 0 & 0 & 0 \\
0 & 0 & 0 & m_{f 4} & 0 & 0 & 0 \\
\hdashline 0 & 0 & 0 & 0 & m_{r 1} & 0 & 0 \\
0 & 0 & 0 & 0 & 0 & m_{r 2} & 0 \\
\hline 0 & 0 & 0 & 0 & 0 & 0 & m_{I T D}
\end{array}\right]\left\{\begin{array}{c}
\ddot{q}_{f 1} \\
\ddot{q}_{f 2} \\
\ddot{q}_{f 3} \\
\ddot{q}_{f 4} \\
\ddot{q}_{r 1} \\
\ddot{q}_{r 2} \\
\ddot{x}_{I T D}
\end{array}\right\}+} \\
& {\left[\begin{array}{llll:ll|l}
c_{11} & c_{12} & c_{13} & c_{14} & c_{15} & c_{16} & c_{17} \\
c_{12} & c_{22} & c_{23} & c_{24} & c_{25} & c_{26} & c_{27} \\
c_{31} & c_{32} & c_{33} & c_{34} & c_{35} & c_{36} & c_{37} \\
c_{41} & c_{42} & c_{43} & c_{44} & c_{45} & c_{46} & c_{47} \\
\hdashline c_{51} & c_{52} & c_{53} & c_{54} & c_{55} & c_{56} & c_{57} \\
c_{61} & c_{62} & c_{63} & c_{64} & c_{65} & c_{66} & c_{67} \\
\hline c_{71} & c_{72} & c_{73} & c_{74} & c_{75} & c_{76} & c_{77}
\end{array}\right]\left\{\begin{array}{l}
\dot{q}_{f 1} \\
\dot{q}_{f 2} \\
\dot{q}_{f 3} \\
\dot{q}_{f 4} \\
\hdashline \dot{q}_{r 1} \\
\dot{q}_{r 2} \\
\hdashline \dot{x}_{I T D}
\end{array}\right\}+\left[\begin{array}{llll:ll|l}
k_{11} & k_{12} & k_{13} & k_{14} & k_{15} & k_{16} & k_{17} \\
k_{12} & k_{22} & k_{23} & k_{24} & k_{25} & k_{26} & k_{27} \\
k_{31} & k_{32} & k_{33} & k_{34} & k_{35} & k_{36} & k_{37} \\
k_{41} & k_{42} & k_{43} & k_{44} & k_{45} & k_{46} \\
\hdashline k_{51} & k_{52} & k_{53} & k_{54} & k_{55} & k_{56} & k_{57} \\
k_{61} & k_{62} & k_{63} & k_{64} & k_{65} & k_{66} & k_{67} \\
\hdashline k_{71} & k_{72} & k_{73} & k_{74} & k_{75} & k_{76} & k_{77}
\end{array}\right]\left\{\begin{array}{l}
q_{f 1} \\
q_{f 2} \\
q_{f 3} \\
q_{f 4} \\
\hdashline q_{r 1} \\
q_{r 2} \\
x_{I T D}
\end{array}\right\}-} \\
& k c u b e^{*}\left[\begin{array}{l}
k c_{1} \\
k c_{2} \\
k c_{3} \\
k c_{4} \\
\bar{k} c_{5} \\
k c_{6} \\
k c_{7}
\end{array}\right]+k c m *\left[\begin{array}{l}
k c m_{1} \\
k c m_{2} \\
k c m_{3} \\
k c m_{4} \\
\overline{k c m} \\
k c m_{5} \\
k c m_{7}
\end{array}\right]=\left\{\begin{array}{l}
\phi_{f 1}(7) \\
\phi_{f 2}(7) \\
\phi_{f 3}(7) \\
\phi_{f 4}(7) \\
-\phi_{r 1}(8) \\
\phi_{r 2}(8)
\end{array}\right\} * f(t, \omega, \alpha) \text {. }
\end{aligned}
$$

where the damping coefficients are 


$$
\begin{aligned}
& c_{11}=2 * \zeta_{f 1} * \omega_{n f 1}+c_{I T D} *\left(\phi_{f 1}(5)\right)^{2}, c_{12}=c_{21}=c_{I T D} * \phi_{f 1}(5) * \phi_{f 2}(5), \\
& c_{13}=c_{31}=c_{I T D} * \phi_{f 1}(5) * \phi_{f 3}(5), c_{14}=c_{41}=c_{I T D} * \phi_{f 1}(5) * \phi_{f 4}(5), c_{17}=c_{71}-c_{I T D} * \phi_{f 1}(5), \\
& c_{22}=2 * \zeta_{f 2} * \omega_{n f 2}+c_{I T D} *\left(\phi_{f 2}(5)\right)^{2}, c_{23}=c_{32}=c_{I T D} * \phi_{f 2}(5) * \phi_{f 3}(5), \\
& c_{24}=c_{42}=c_{I T D} * \phi_{f 2}(5) * \phi_{f 4}(5), c_{27}=c_{72}=-c_{I T D} * \phi_{f 1}(5), \\
& c_{33}=2 * \zeta_{f 3} * \omega_{n f 3}+c_{I T D} *\left(\phi_{f 3}(5)\right)^{2}, c_{34}=c_{43}=c_{I T D} * \phi_{f 3}(5) * \phi_{f 4}(5), \\
& c_{37}=c_{73}=-c_{I T D} * \phi_{f 3}(5), c_{44}=2 * \zeta_{f 4} * \omega_{n f 4}+c_{I T D} *\left(\phi_{f 4}(5)\right)^{2}, c_{47}=c_{74}-c_{I T D} * \phi_{f 4}(5), \\
& c^{55}=2 * \zeta_{r 1} * \omega_{n r 1}, \quad c_{66}=2 * \zeta_{r 2} * \omega_{n r 2}, c_{77}=c_{I T D},
\end{aligned}
$$

and all other damping coefficients are zero. Accordingly, the stiffness coefficients are

$$
\begin{aligned}
& k_{11}=k_{f 1}+k_{I T D} *\left(\phi_{f 1}(5)\right)^{2}, k_{12}=k_{21}=k_{I T D} * \phi_{f 1}(5) * \phi_{f 2}(5), \\
& k_{13}=k_{31}=k_{I T D} * \phi_{f 1}(5) * \phi_{f 3}(5), k_{14}=k_{41}=k_{I T D} * \phi_{f 1}(5) * \phi_{f 4}(5), \\
& k_{17}=k_{71}-k_{I T D} * \phi_{f 1}(5), k_{22}=k_{f 2}+k_{I T D} *\left(\phi_{f 2}(5)\right)^{2}, k_{23}=k_{32}=k_{I T D} * \phi_{f 2}(5) * \phi_{f 3}(5), \\
& k_{24}=k_{42}=k_{I T D} * \phi_{f 2}(5) * \phi_{f 4}(5), k_{27}=k_{72}=-k_{I T D} * \phi_{f 1}(5), k_{33}=k_{f 3}+k_{I T D} *\left(\phi_{f 3}(5)\right)^{2}, \\
& k_{34}=k_{43}=k_{I T D} * \phi_{f 3}(5) * \phi_{f 4}(5), k_{37}=k_{73}=-k_{I T D} * \phi_{f 3}(5), k_{44}=k_{f 4}+k_{I T D} *\left(\phi_{f 4}(5)\right)^{2}, \\
& k_{47}=k_{74}-k_{I T D} * \phi_{f 4}(5), k_{55}=k_{r 1}, k_{66}=k_{r 2}, k_{77}=k_{I T D},
\end{aligned}
$$

and all other stiffness are zero. The nonlinear components are

$$
\begin{aligned}
& k c_{1}=\left(\left(\phi_{f 1}\right)^{2}+\phi_{f 1} * \phi_{f 2}+\phi_{f 1} * \phi_{f 3}+\phi_{f 1} * \phi_{f 4}-\phi_{f 1}\right)^{3}, \\
& k c_{2}=\left(\left(\phi_{f 2}\right)^{2}+\phi_{f 2} * \phi_{f 1}+\phi_{f 2} * \phi_{f 3}+\phi_{f 2} * \phi_{f 4}-\phi_{f 2}\right)^{3},
\end{aligned}
$$




$$
\begin{aligned}
& k c_{3}=\left(\left(\phi_{f 3}\right)^{2}+\phi_{f 3} * \phi_{f 2}+\phi_{f 3} * \phi_{f 1}+\phi_{f 3} * \phi_{f 4}-\phi_{f 3}\right)^{3}, \\
& k c_{4}=\left(\left(\phi_{f 4}\right)^{2}+\phi_{f 4} * \phi_{f 2}+\phi_{f 4} * \phi_{f 3}+\phi_{f 4} * \phi_{f 1}-\phi_{f 4}\right)^{3}, k c_{5}=k c_{6}=0, k c_{7}=1, \\
& k c m_{5}=\left(\left(\phi_{r 1}\right)^{2}+\phi_{r 1} * \phi_{r 2}\right)^{3}, \quad k c m_{6}=\left(\left(\phi_{r 2}\right)^{2}+\phi_{r 1} * \phi_{r 2}\right)^{3}, \text { and } \\
& k c m_{1}=k c m_{2}=k c m_{3}=k c m_{4}=k c m_{7}=0 .
\end{aligned}
$$

All these equations use $\varphi$ and $q$ which are the eigenvectors and modal coordinates of the front shaft modes previously noted. In addition, the subscripts $f 1-4$ are attributed to the front driveshaft model mode shapes 1-4, $r 1-2$ are attributed to the rear driveshaft model mode shapes 1-2. The modal masses $(m)$ stiffnesses $(k)$, damping ratios $(\zeta)$, natural frequencies $\left(\omega_{n}\right)$ and coordinates $(q)$ are key characteristics from each driveshaft's modal equations of motion. The applied forcing function of the secondary moment, $f(t, \omega, \alpha)$ is transformed to the modal domain by multiplication of the eigenvector $(\varphi)$ of the respective modes at the position of the universal joint. The cubic coefficient of kcube is the measured linear cubic stiffness term from the ITD. The second cubic coefficient of $\mathrm{kcm}$ is the cubic moment stiffness as measured on the center bearing.

Additional linear stiffness and non-linear stiffness terms were added to each modal equation of motion. Likewise, the center bearing support of the rear driveshaft cubic moment non-linear stiffness was added using the same modal modification techniques. This generated nonlinear modal coordinate equations of motion for the front and rear driveshafts respectively. 


\section{Modal Coupling}

Up to this point, the system of equations has had 2 separate coupled systems based on the front and rear driveshaft. The two sets must be coupled together to create the final driveshaft system. Therefore, the driveshafts are then coupled together with a hinge joint using modal coupling techniques at the location of the universal joint. A standard pin couple was performed at this point. To accomplish this, the transformation matrix was first defined in the physical coordinate system. Subsequently, this coupling was then transformed using the same physical to modal transformation matrices used for the modal equations of motion derivation. This was necessary to couple the equations at this point to ensure adequate freedom of vibration across the universal joint angle. Following approach outlined by Cuppens et al and is utilized in LMS Virtual.lab analysis software uses the derivation of the transformation matrix[44]. The transformation matrix is developed for a rigid connection by the modal transformation of both modal systems of the physical connection between the two systems. For this case, the transformation is defined through submatricies

$$
\begin{aligned}
T d & =\left[\begin{array}{ll}
\phi_{f 1}(7 y) & \phi_{f 2}(7 y) \\
\phi_{f 1}(7 x) & \phi_{f 2}(7 x)
\end{array}\right] \\
T i & =\left[\begin{array}{lllll}
\phi_{f 3}(7 y) & \phi_{f 4}(7 y) & \phi_{r 1}(8 y) & \phi_{r 2}(8 y) & 0 \\
\phi_{f 3}(7 x) & \phi_{f 4}(7 x) & \phi_{r 1}(8 x) & \phi_{r 2}(8 x) & 0
\end{array}\right]
\end{aligned}
$$

combine to form the final coupling transformation matrix 


$$
T=\left[\begin{array}{ccccc}
\frac{-T i(1,1)}{T d(1,1)} & \frac{-T i(1,2)}{T d(1,1)} & \frac{-T i(1,3)}{T d(1,1)} & \frac{-T i(1,4)}{T d(1,1)} & \frac{-T i(1,5)}{T d(1,1)} \\
\frac{-T i(2,1)}{T d(2,2)} & \frac{-T i(2,2)}{T d(2,2)} & \frac{-T i(2,3)}{T d(2,2)} & \frac{-T i(2,4)}{T d(2,2)} & \frac{-T i(2,5)}{T d(2,2)} \\
1 & 0 & 0 & 0 & 0 \\
0 & 1 & 0 & 0 & 0 \\
0 & 0 & 1 & 0 & 0 \\
0 & 0 & 0 & 1 & 0 \\
0 & 0 & 0 & 0 & 1
\end{array}\right] \text { [44] }
$$

The transformation Matrix was then applied to all matrices. The application follows the standard transformation matrix as applied to the mass, stiffness and damping matrices,

$$
\begin{aligned}
& M c=T^{T} M T \\
& K c=T^{T} K T \\
& C c=T^{T} C T
\end{aligned} .
$$

The same transformation can be applied to the nonlinear system prior to computing the cube portion, as it can be listed as a matrix of coefficient representative of the transformation from the physical domain.

The final system of equations had 7 degrees of freedom, with two dependant modes from the modal coupling to produce 5 modal degrees of freedom for the final simulation. Care was taken during the assembly process as the driveshafts, ITD, and universal joint forces were developed using multiple coordinate systems, and transformation into a global coordinate system was necessary. The universal joint force was added to the node where the universal joint connects the front and rear driveshaft. 
Once again physical to modal coordinate system transformation was necessary resulting in a forcing component in each of the equations of motion.

In summary, the final modal equations of motion include the nonlinear internal tuned damper stiffness, nonlinear center bearing moment stiffness, and have freedom across the universal joint angle direction. In addition, the excitation is the full function formula and includes possible parametric excitation due to the universal joint angles changing due to the vibration. The following equation presents the form of the final modal equations of motion used for the subsequent simulation analysis

$M \ddot{q}+C \dot{q}+K q+K_{c u b i c} q^{3}=F(q, \alpha, t)$.

where $\mathrm{M}, \mathrm{C}, \mathrm{K}$ are the linear modal mass, damping, and stiffness matrices, $\mathrm{K}_{\text {cubic }}$ is the cubic stiffness, and $F(q, \alpha, t)$ is the modal forcing vector that is dependent on the modal state, misalignment angle, and time.

\section{Summary of Model Correlation and Simulation Results}

The final results for analytical linear model normal modes analysis (removing the nonlinear terms) and the experimental modal analysis results are presented in Table 4. The linear modal analysis and the experimental modal analysis are comparable as they both assume linearity of the equations of motion. There is good agreement of the normal mode analysis and the test response up to the frequency of the jump. The rear driveshaft bending mode has a significant discrepancy of identified natural frequencies. Upon review of the data, the rear axle spindle has a significant cantilevered overhang of the 
flange attachment to the universal joint flange on the driveshaft. This overhang adds flexibility that was not included in the Normal mode analysis, which includes only the driveshaft stiffness influences.

Table 4

Comparison of Analytical and Experimental Natural Frequencies

\begin{tabular}{lll}
\hline Mode & Normal Modes & Test Response \\
\hline Center bearing bounce & 15.6 & $16.5 \mathrm{~Hz}$ \\
Front Driveshaft/ITD lower & 106 & $95 \mathrm{~Hz}$ \\
Front Driveshaft/ITD upper & 162 & $146 \mathrm{~Hz}$ \\
Rear driveshaft bending & 233 & $162 \mathrm{~Hz}$ \\
Front driveshaft second bending & 457 & $385 \mathrm{~Hz}$ \\
\hline
\end{tabular}

The simulation of a ramp up similar to the experiment resulted in a nonlinear jump occurring at approximately 2100 RPM. First a simulation with the full force input produced a nonlinear jump. The physical responses of both the internal tuned damper and the driveshaft at the location of the internal tuned damper are plotted in Fig. 32.

The jump is predominant on the front driveshaft/ITD upper natural frequency identified in the normal modes analysis. In addition to the ITD and shaft, the freedom across the universal joint connection also shows the nonlinear jump. Their responses of the front and rear driveshaft angles at the universal joint are shown in Fig. 33 with each individual angle presented first, and then the difference between the angles presented in the last plot. 


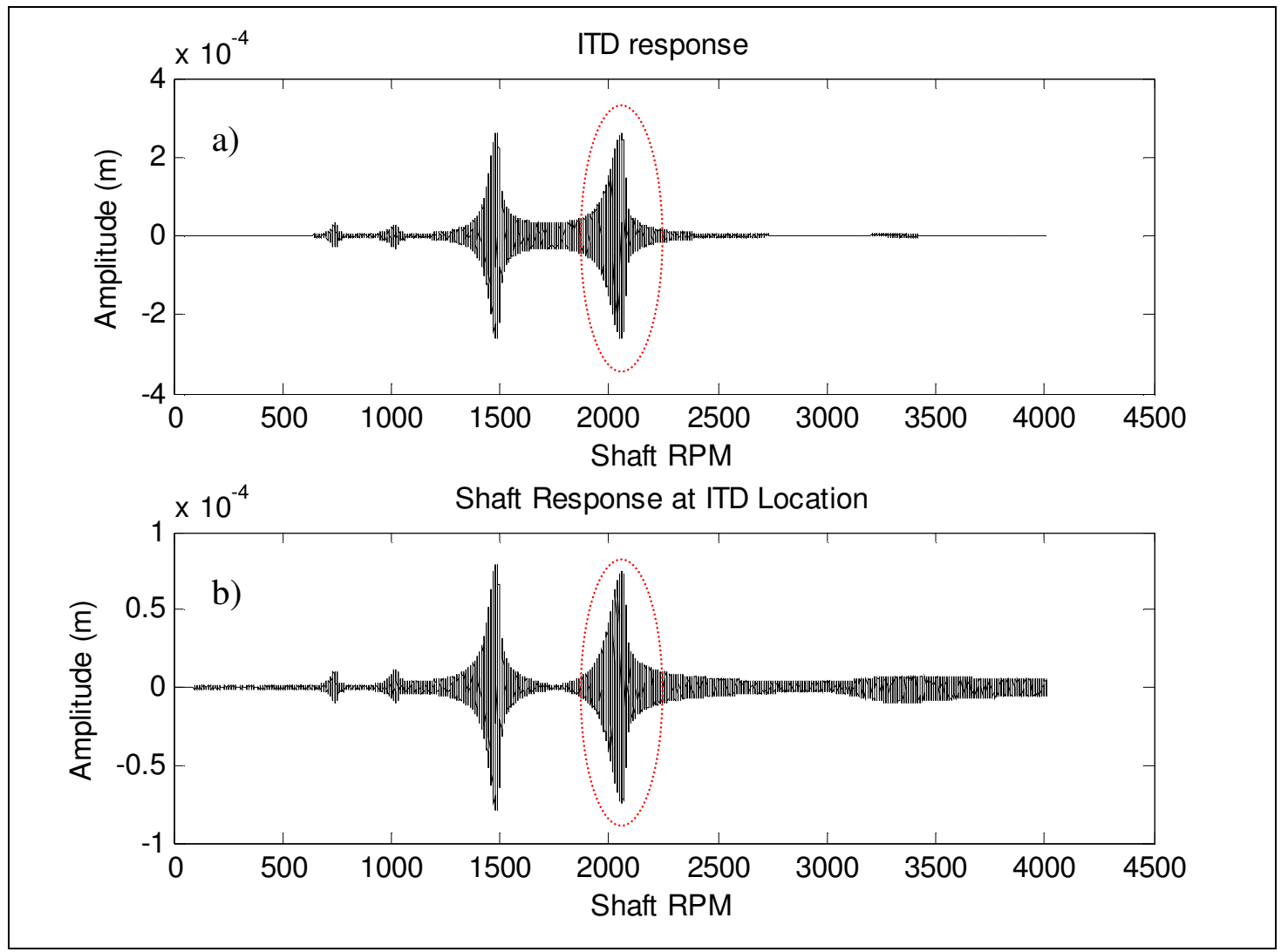

Fig. 32. Simulation Run Up Vibrations at a) ITD and b) Driveshaft at Shaft to ITD Connection Node. 


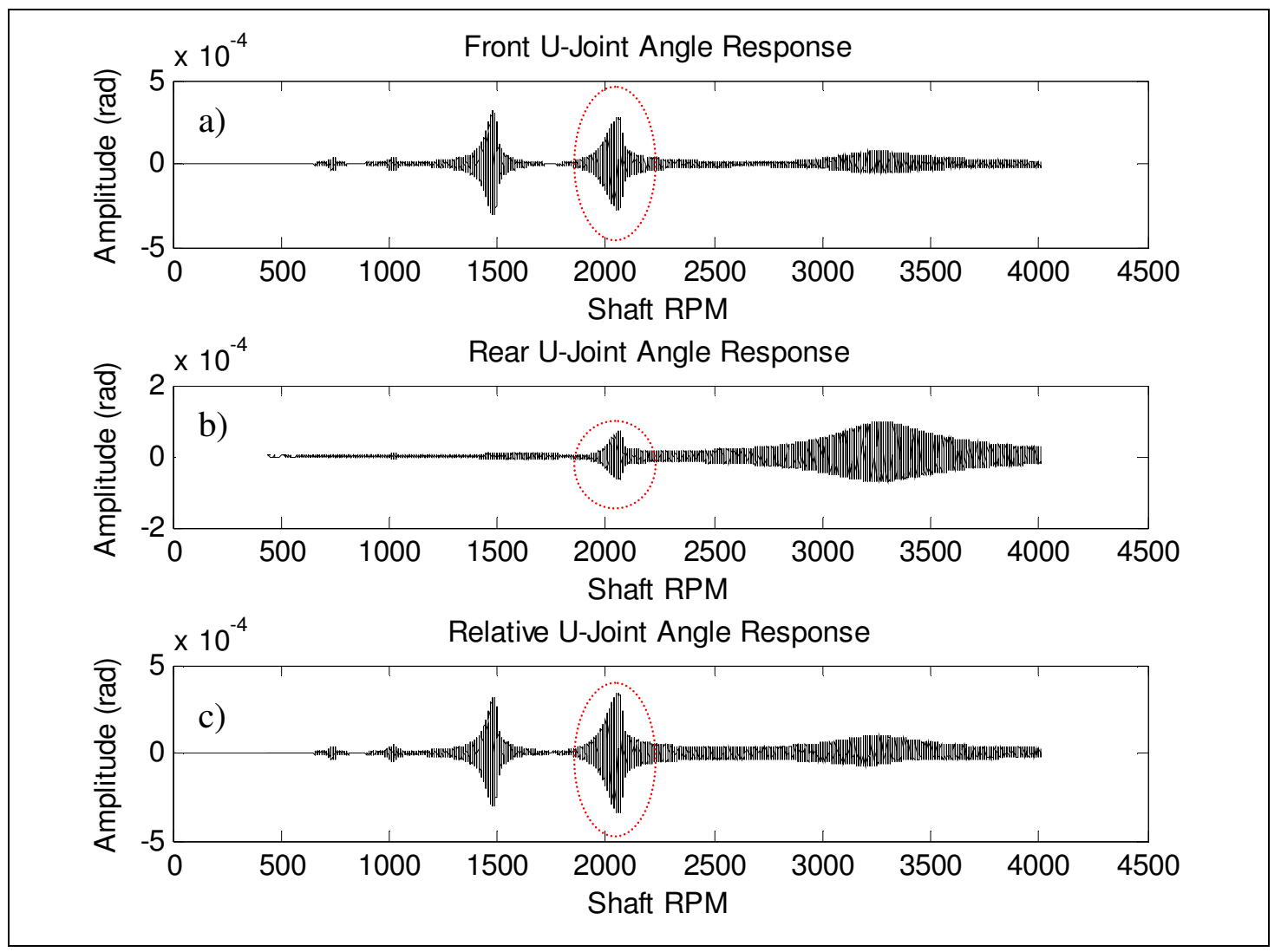

Fig. 33. Angle Response at Center Universal Joint for a) Front Shaft Angle, b) Rear Shaft Angle, and c) the Total Relative Angle Between Shafts.

The jump is also apparent in the universal joint angles, reflecting the front shaft in the front universal joint, but a slightly different response in the rear shaft angle. The response of the rear universal joint angle is similar in shape as the response on the center bearing $\mathrm{x}$ direction lining up with the rear driveshaft, and $\mathrm{z}$ direction for the front driveshaft for the $4^{\text {th }}$ order response from the experimental test results. Due to the system support on the center bearing on the rear shaft, the observed measured results would compare best with those at the center bearing. Therefore, comparisons of the 
experimental results to the simulated results, Fig. 34 to Fig. 33 a), show some interesting features.

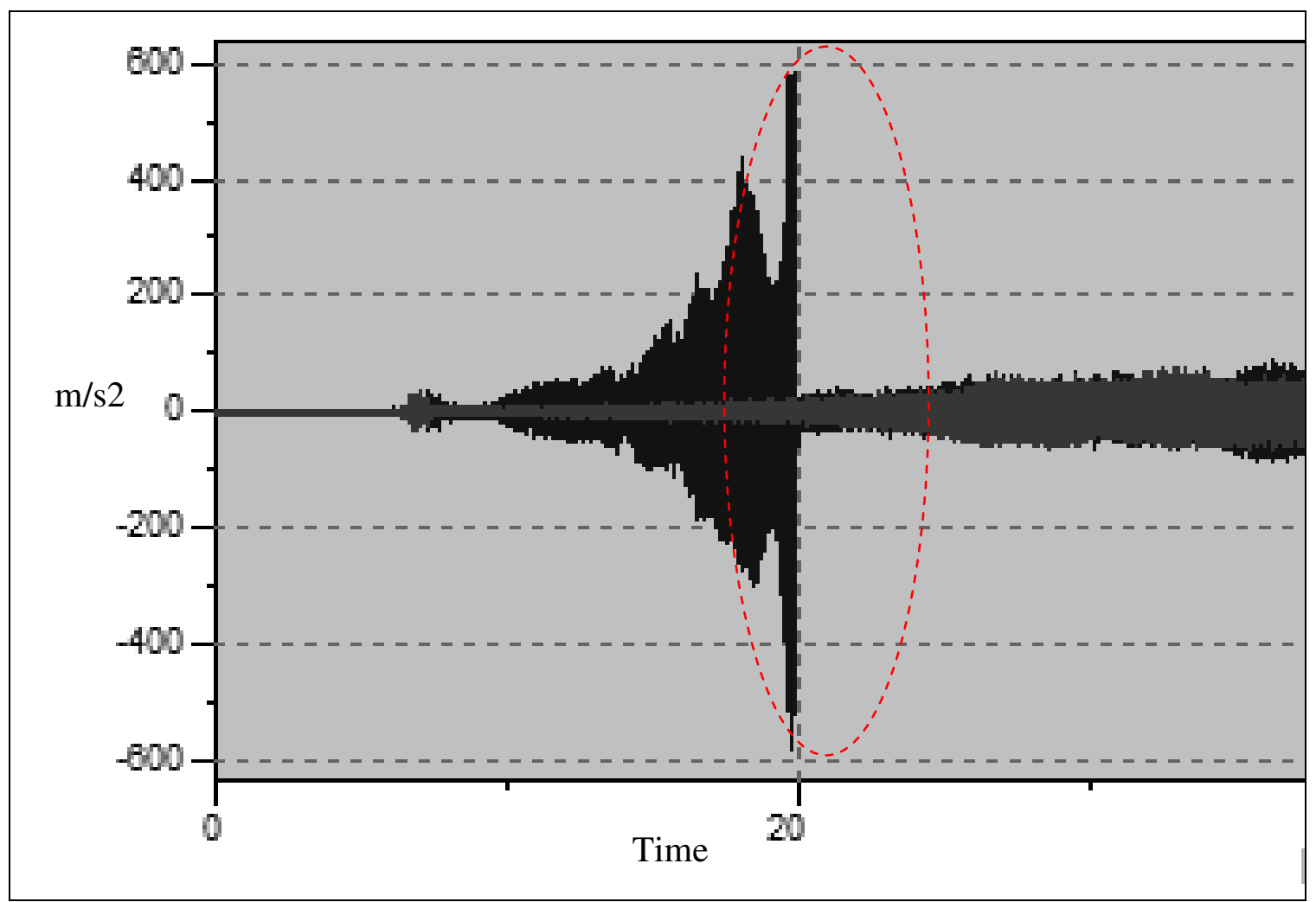

Fig. 34. Measured Center Bearing Response Top X-dir.

Two peaks are present in both results, but the experimental measurement has the peaks closer together. These results align with the small differences between the normal modes and test results of the identification of the natural frequencies, see Table 2.

Figures $33 \mathrm{~b}$ and 35 show a similar behavior with two peaks, and greater separation in the simulation results due to the results of the normal modes analysis. The simulation results are displacements while the measured results are acceleration. This 
will change the shapes of the responses but not the separations in peak amplitude speeds. The responses of the universal joints are dominated by the $4^{\text {th }}$ order excitation progressing through the range of the front shaft bending and internal tuned damper tuning frequency.

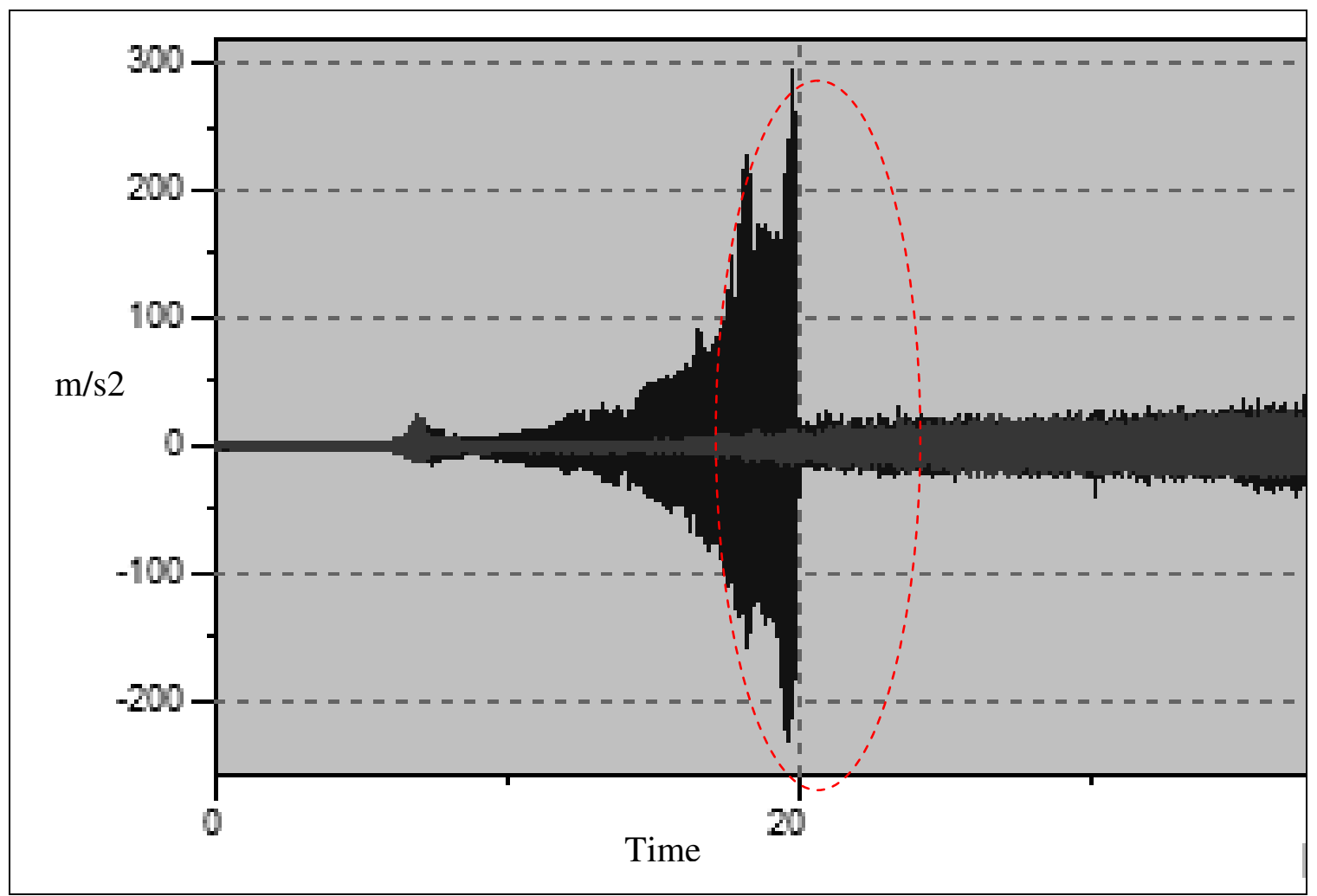

Fig. 35. Measured Center Bearing Top Z-dir.

Spectral analysis of the simulation response shows the significant order content, and the influence of the natural frequencies. A plot of the rear shaft universal joint response frequency spectrum is pictured in Fig. 36. 


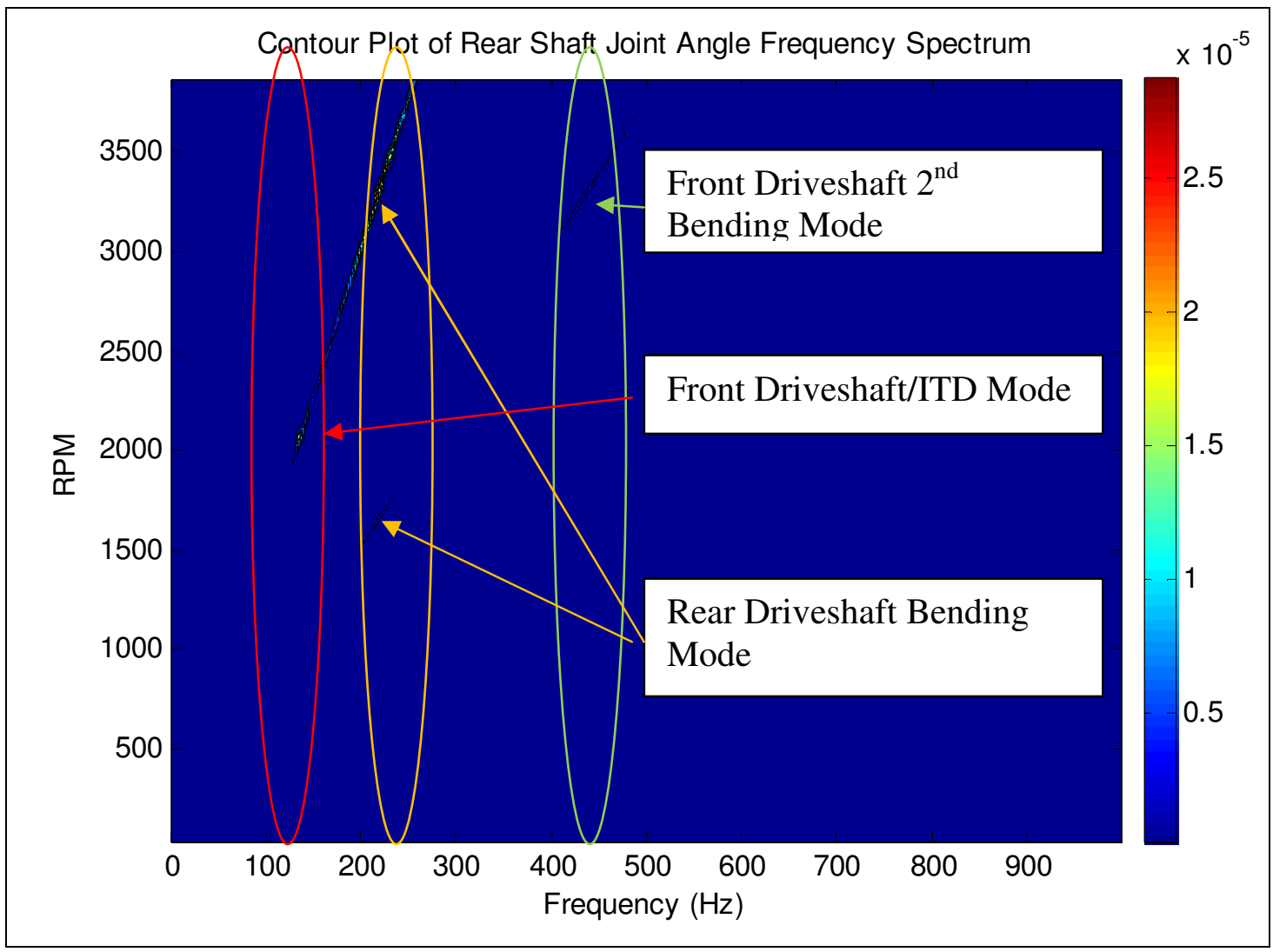

Fig. 36. Contour Plot of Simulated Vibrations of the Rear Shaft Joint Angle.

The response clearly shows the jump occurring at around the $162 \mathrm{~Hz}$ front driveshaft/ITD natural frequency. In addition it can be noted that the $8^{\text {th }}$ order also excites this mode to a small degree. The $8^{\text {th }}$ order excitations also excite the rear driveshaft bending and front driveshaft second bending modes as well.

The simulation was completed with the derived full secondary moment equation, Fourier series as derived, and a truncated Fourier series with only $4^{\text {th }}$ order excitation. A comparison of the ITD response is presented in Fig. 37. 


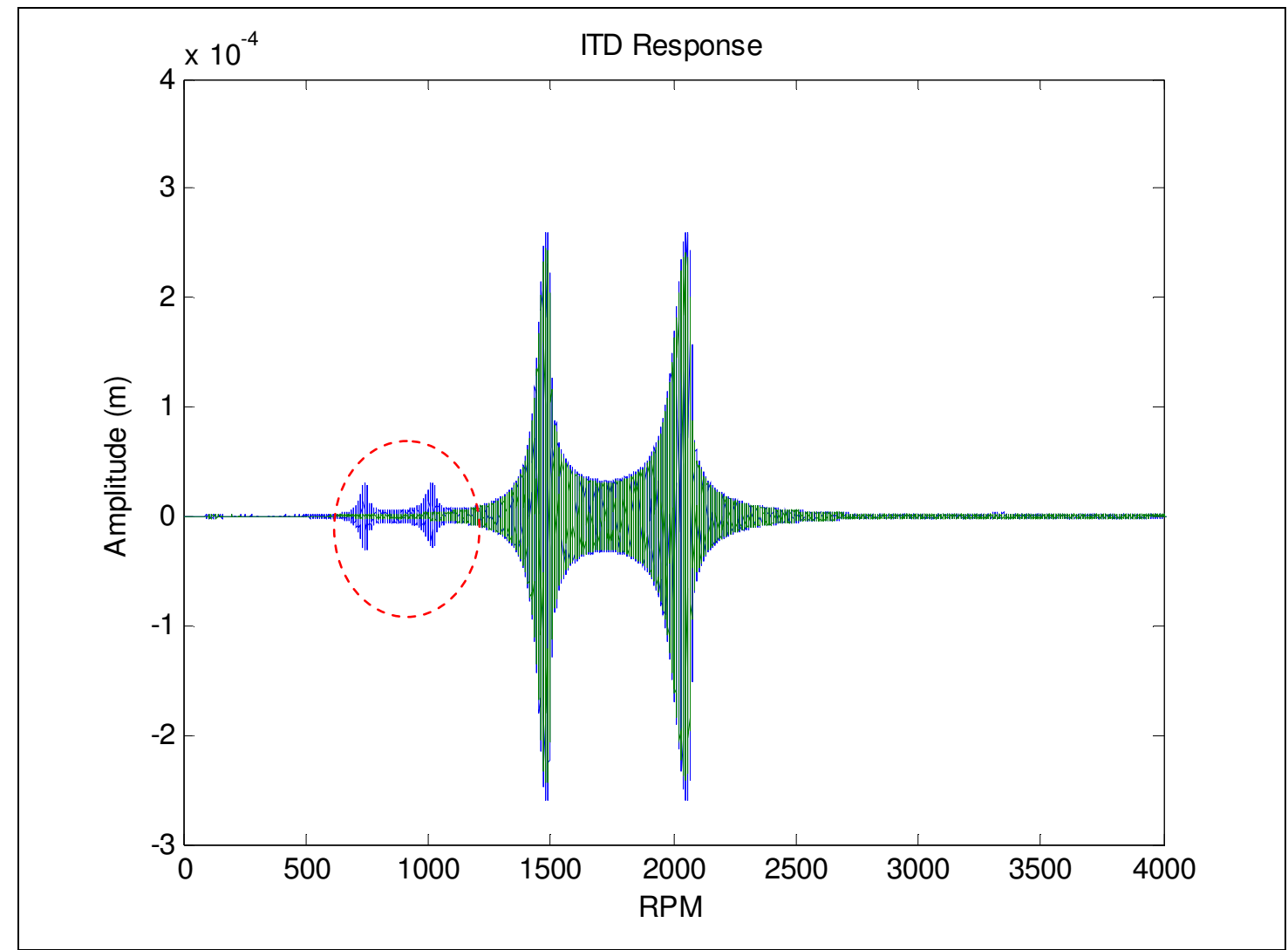

Fig. 37. ITD Response Comparison of Full Force Excitation (blue) and Fourier 4th Order Excitation (green).

The results showed little difference in the simulated displacements. The jump at approximately 2100 RPM is still present. The Fourier excitation does not include the small increased vibrations as the $8^{\text {th }}$ order passes through the front driveshaft/ITD modes. This is due to the Fourier excitation only included the $4^{\text {th }}$ order excitation term. Subsequently the same comparison is completed for the location on the driveshaft in Fig. 38. 


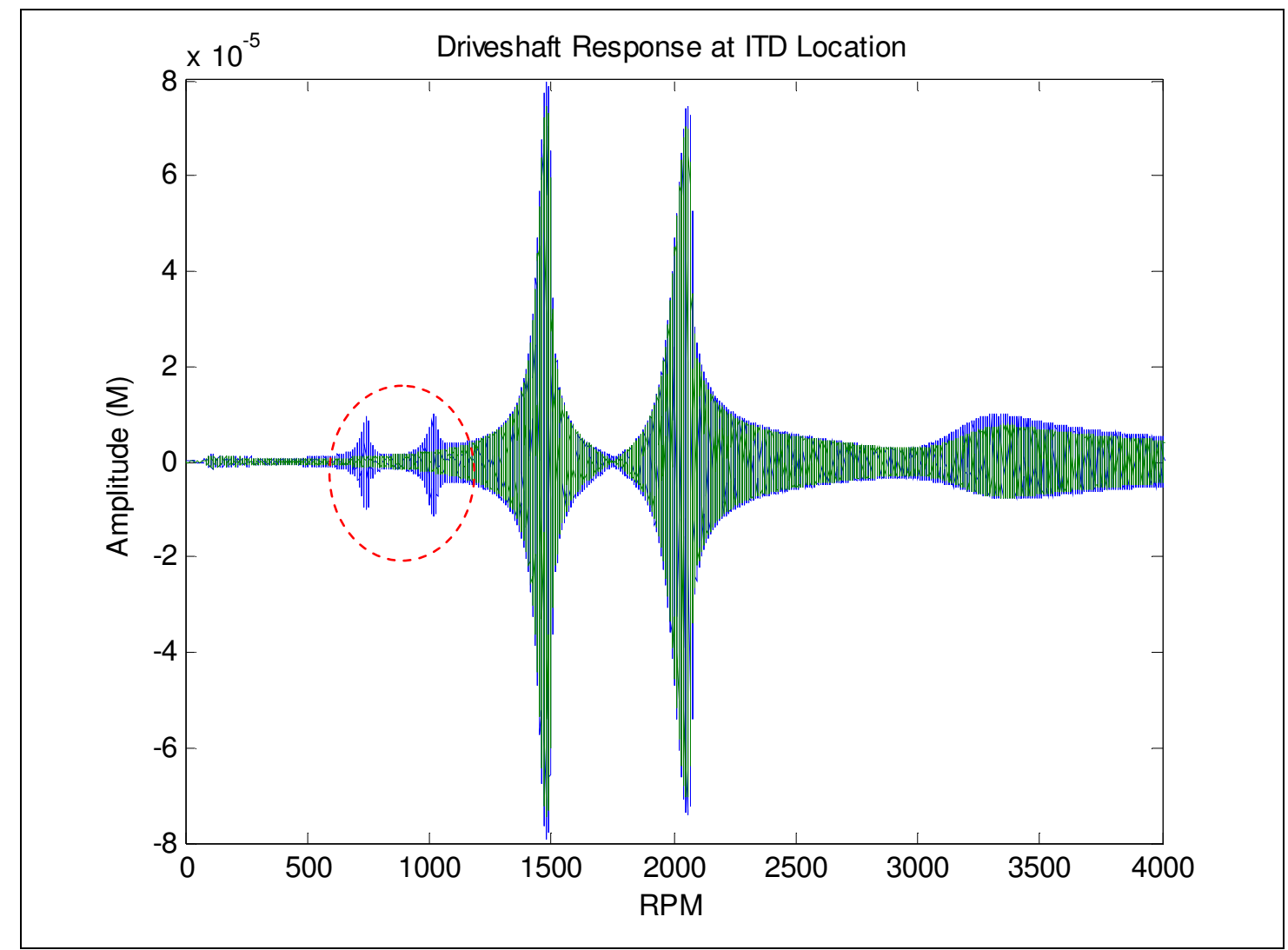

Fig. 38. Driveshaft Response Comparison of Full Force Excitation (blue) and Fourier 4th Order Excitation (green).

Once again, similar results proved little difference around the 2100 RPM jump range. There are differences where the $8^{\text {th }}$ order excitations passing through the front driveshaft/ITD modes. This is due to the simulation of these excitation orders with the Fourier series including only the $4^{\text {th }}$ order. Frequency spectral analysis shows that only $4^{\text {th }}$ order response is generated in Fig. 39. 


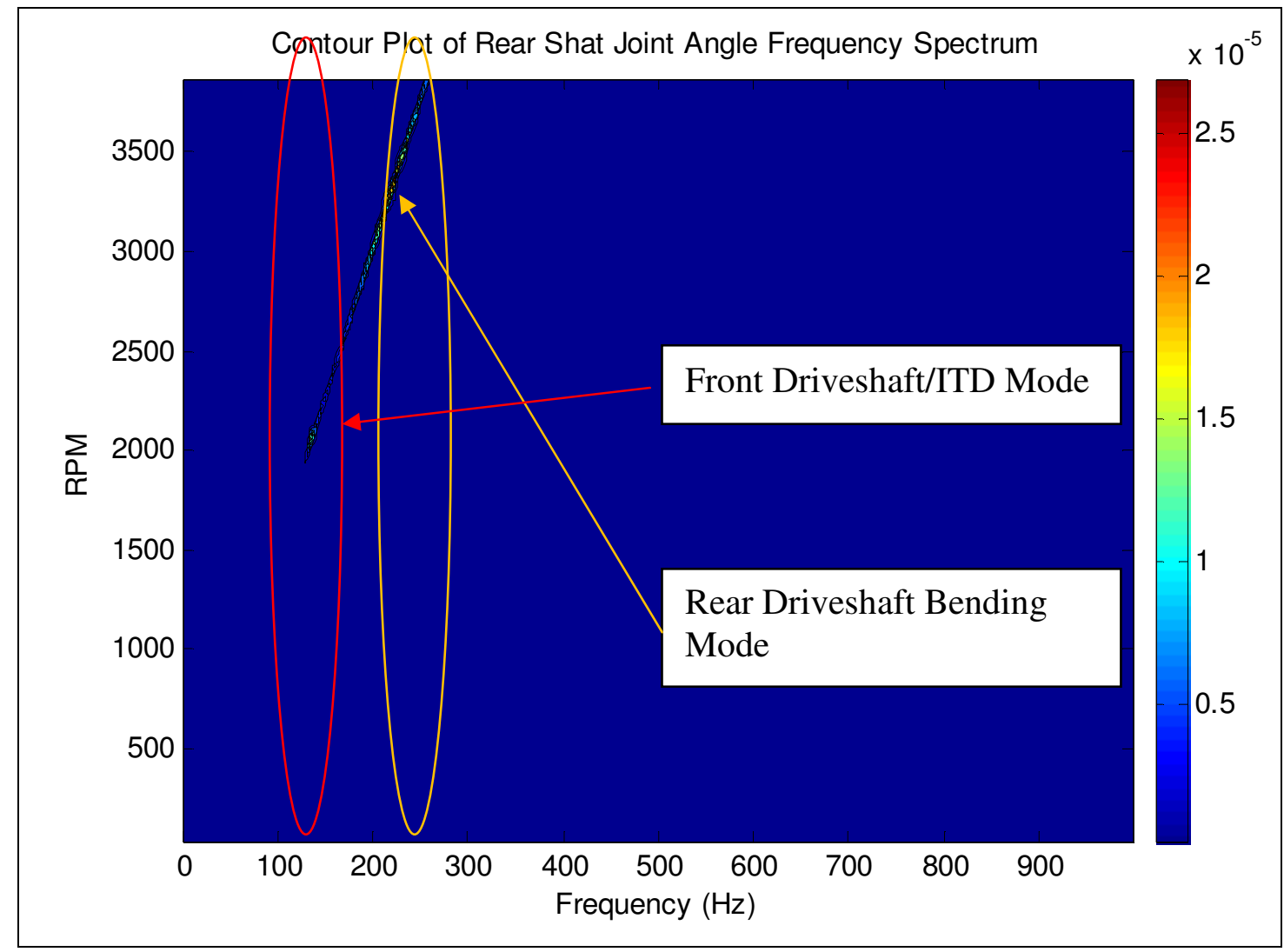

Fig. 39. Fourier Series with Only $4^{\text {th }}$ Order Contribution Contour Plot.

The spectrum shows only the $4^{\text {th }}$ order response terms. The same nonlinear jump was identified, and the same excitation of the same mode shapes is present.

Therefore, either the full derived force or the Fourier approximation equations are sufficient for use in analysis with the same misalignment angle. The nonlinear stiffness of the internal tuned damper was removed, and the results produced a standard linear response without the jump as pictured in Fig. 40. 


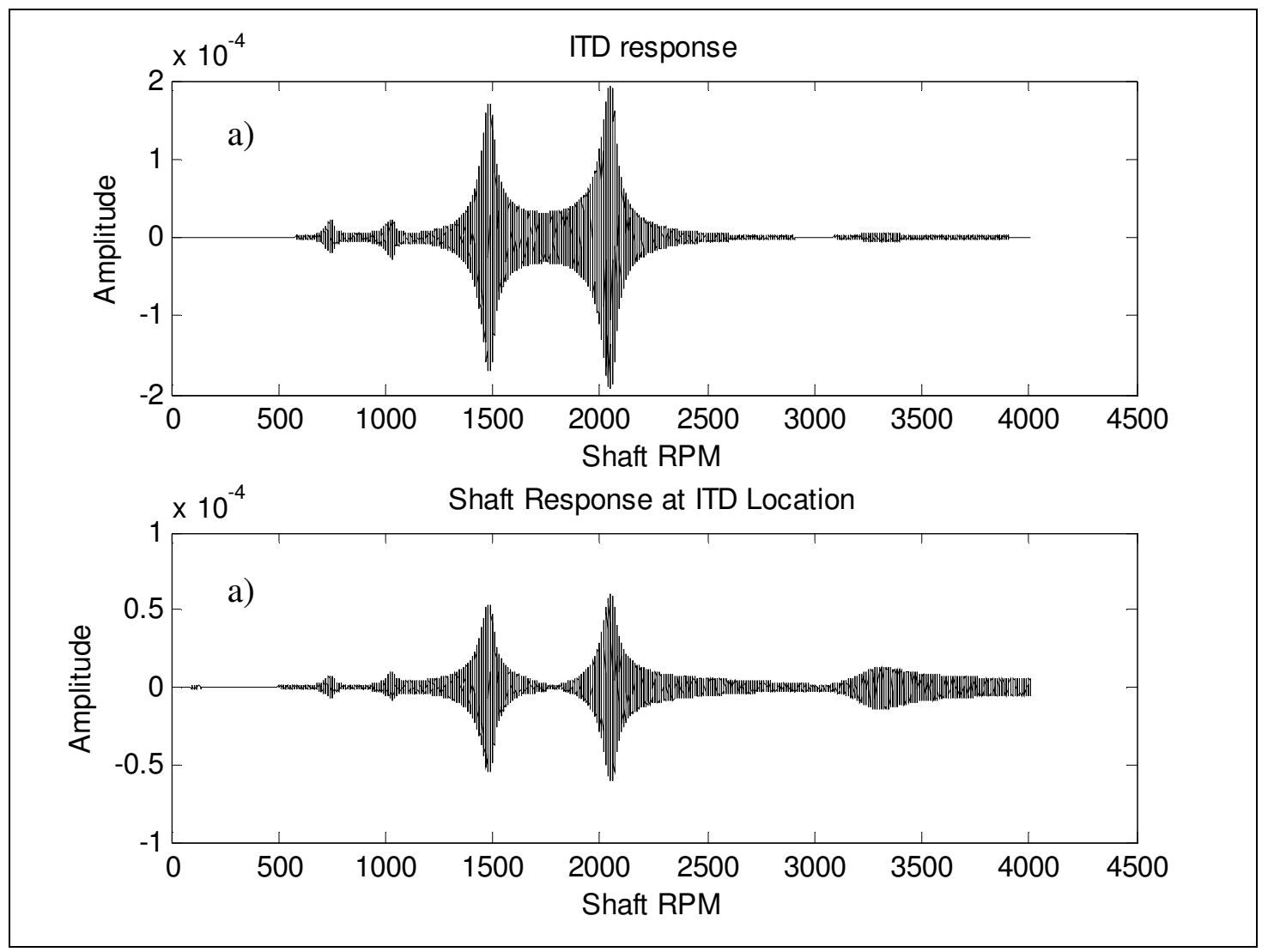

Fig. 40. Linear Simulation Results of a) ITD and b) Driveshaft Response at ITD Location.

The response with no nonlinear stiffness of the ITD produces linear response. This linear response includes possible parametric excitation of the forced secondary moment, and the nonlinear stiffness of the center bearing. Therefore, the nonlinear ITD stiffness is responsible for the jump, and not parametric excitation or resonance. Subsequently, the experimental data presented previously in Fig. 11 with the drive up portion (0-35 seconds) showing a significant jump, but a subsequent jump during the run down portion (35-70 seconds) produced a significantly different level, and response 
through the resonance region. This response is a symptom of nonlinear response and a parametric excitation would have similar responses in both conditions.

Additional runs at various levels of nonlinearity were made to determine the minimum level of nonlinearity that produces a noticeable jump. This incipient level for the 4 degree angle case was at $25 \%$ of the measured nonlinearity of the internal tuned damper stiffness. From the force input dependency on misalignment angle, the minimum level of nonlinearity to produce a jump would vary inversely with the angle of misalignment.

The effect of reducing damping produced the expected results of extending out the nonlinear region to a higher frequency before the jump occurs. A comparison of a run up and coast down with low damping is plotted in Fig. 41.

The developed model shows decent correlation to the recorded physical system modal and operating data. The analytical model comes within reasonable variation of the system due to possible discrepancies of system constraints. Simulation of the analytical produced the nonlinear jump. Comparing certain points on the center bearing, the analytical and physical operating responses show similar results. 


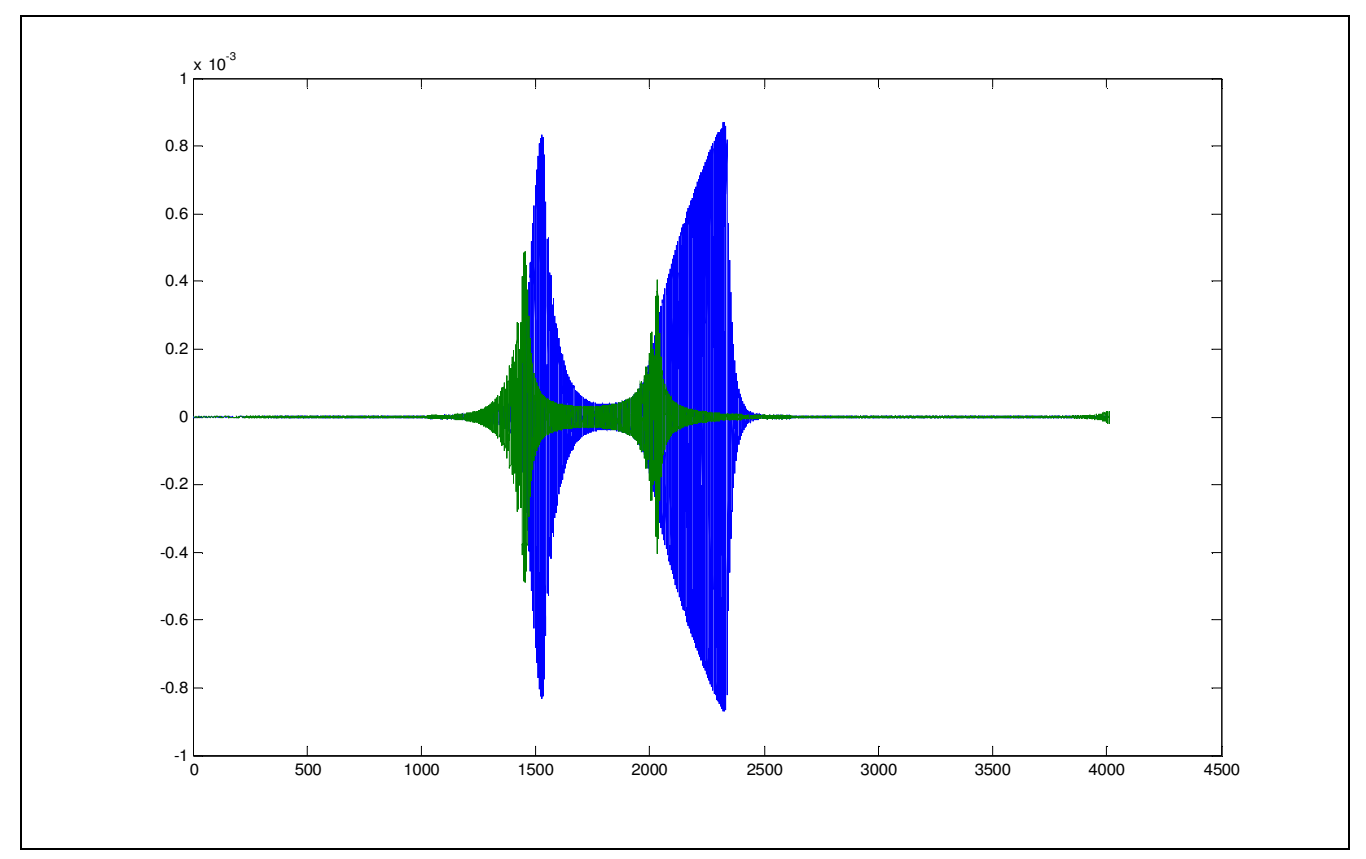

Fig. 41. Plot of the Simulated Response with Low Damping During Run Up (blue) and Run Down (green).

Simulation with the full derived force and the Fourier series approximation provided the same results. The ability to use the Fourier series, and truncation to the $4^{\text {th }}$ order will be beneficial in subsequent analysis. From the simulation results, the truncation of the Fourier series, including only the $4^{\text {th }}$ order excitation helped explain the $4^{\text {th }}$ order excitation is responsible for the excitation to the nonlinear jump.

The analytical model proved that the ITD nonlinear stiffness was the key characteristic of the nonlinear jump. Removal of the stiffness, but inclusion of parametric excitation, and the nonlinear center bearing stiffness produced a typical linear response. The jump was still identifiable in the response at even a $25 \%$ of the measured nonlinear stiffness. Lowering damping increased the range of amplified response prior 
to the nonlinear jump. All these characteristics confirm that the ITD nonlinear stiffness is the source of the nonlinear response. 


\section{CHAPTER V}

\section{NONLINEAR SYSTEM ANALYSIS}

Understanding of the derived dynamic nonlinear system and its subsequent nonlinear behavior is obtained through scrutiny of the system by multiple methods to determine its characteristics. The completed time based simulation verifies the correlation and validity of the analytical model as compared to the physical system. Therefore, the system of equations has been proven adequate for application to nonlinear analysis techniques.

The system of equations includes two major factors influencing forcing function, speed and angle. A third factor of rotational inertia may be addressed, but as in Eqs. (23-34), the inertial term is only a proportional factor on the input force. Therefore, as only a proportional term its influence is not as interesting as the influence of speed and angle. Therefore, analysis into various settings on speed and angle influence on the dynamic response of the system is conducted. High level results as well as specific conditions with interesting characteristics will be presented.

Techniques will be used to determine the characteristics of vibrations.

Calculation of Poincare maps with respect to speed and angle will identify possible regions of bifurcations and/or chaotic vibrations. Investigation into possible bifurcations of the nonlinear system will determine a whether a common path to chaotic vibrations exist. The same range will be tested for chaotic vibrations by calculating the Lyapunov exponent spectrums. Subsequent spectral analysis will determine the components of the 
unique vibrations verifying the chaotic vibrations. Finally, identification of multiple solutions provides final evidence of the validity of the nonlinear jump identified in both simulation and test.

All these analysis leads to further understanding of this unique real system. It is important to note that the presented nonlinear analysis is completed with a modally coupled nonlinear system. Therefore, application of these methods to a derived system of equations is also unique.

\section{Bifurcation and Poincare}

A fundamental understanding of a nonlinear dynamic system is the reaction to a steady state oscillating function. A linear response will provide only the same frequency of response as the frequency of excitation. A nonlinear system may have other frequency components including super and sub harmonic frequencies. One method used to identify possible variations in frequency is to simulate the system with a constant frequency. The response is recorded at the same point in the period for each cycle. This point is called the first return or the Poincare map[45]. A Poincare Sequence is formed with taking a simulation of multiple points and creating a new series of points. An example of this is pictured in Fig. 42. 


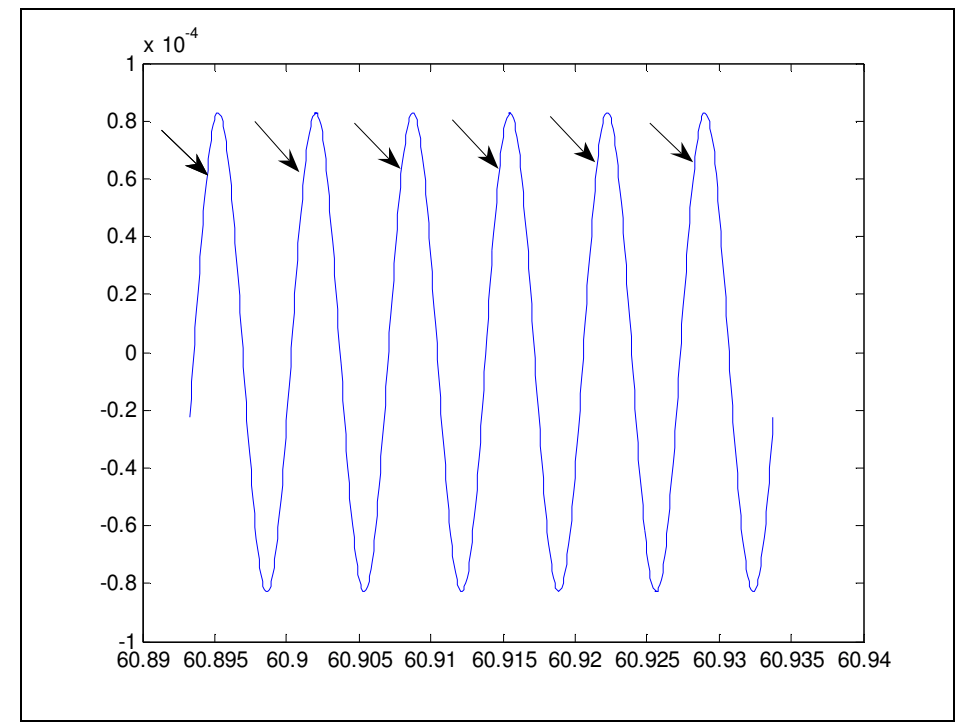

Fig. 42. Sample Response Oscillations with Identification of Points of First Returns.

These first return points can be plotted on a phase plane. The point of first returns will appear as a single point with a linear response. A sample plot of the sample simulation is presented with the point indicated by an arrow in Fig. 43. 


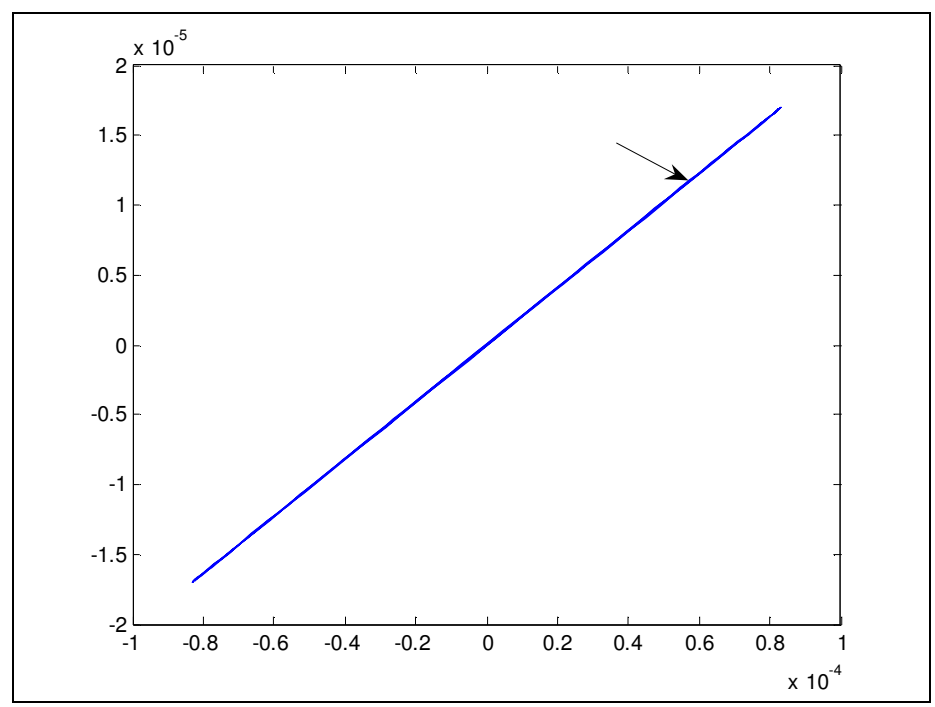

Fig. 43. Phase Phase-Plane Plot of Sample Oscillations.

A nonlinear response with other frequency terms will have a plot with multiple points of single returns, and patterns of the plot can identify sub harmonics or quasi periodic response. Two points would indicate a sub-harmonic (period-2), and three or more points (period-3, period n) may indicate multiple sub-harmonics of the primary excitation. A pattern of points creating a closed loop typically are quasi-periodic to chaotic responses. Therefore, the nonlinear response can be characterized at a single point by the pattern of first returns on the phase-plane diagram.

Plotting the magnitude of the point of first returns across a range of input factors can determine where a nonlinear system stops acting like a linear system, and create multiple frequency outputs from a single frequency input. This diagram is often referred to as a bifurcation diagram. This allows for identification of the points where a period-1 response becomes multiple period responses based on an input factor. 
A range of input speeds with the tested system 4 degree of misalignment angle was simulated to steady state, and then the Poincare sequence was determined and plotted on a bifurcation diagram. The analysis used an initial condition vector of all zeros to start the simulation. The system was simulated to thousands of cycles to allow all transient response to decay and reveal the steady state response. The system was excited with only the $4^{\text {th }}$ order term of the Fourier series. The modal data was transformed to the physical domain to determine the physical response of the front driveshaft where the ITD is attached. The period of identification of the first returns is equivalent to the period of excitation of the $4^{\text {th }}$ order excitation. The $4^{\text {th }}$ order excitations were simulated from 2 to $250 \mathrm{~Hz}$. The results are presented in Fig. 44.

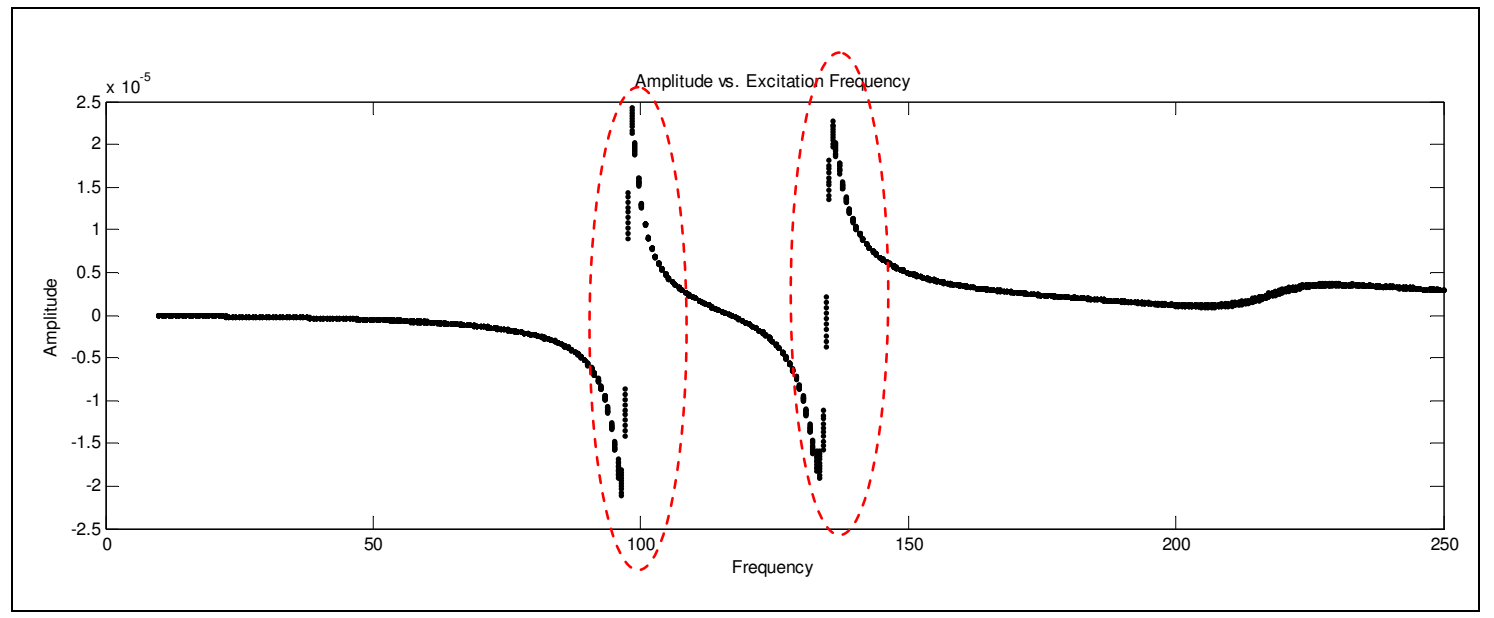

Fig. 44. Bifurcation Diagram with 4 Degree Misalignment Across 4x Input Frequencies of 2-250 Hz with $4^{\text {th }}$ Order Excitation Only. 
The diagram shows mostly a single point response for each input frequency across the range. A couple of frequencies at just below 100 and at just below $150 \mathrm{~Hz}$ show possible multiple first return points. These frequencies are near the front driveshaft/ITD modes previously discussed. Finer frequency resolution and a longer stabilization period for the simulation were completed for frequencies near these resonances. The results are presented in Fig. 45.

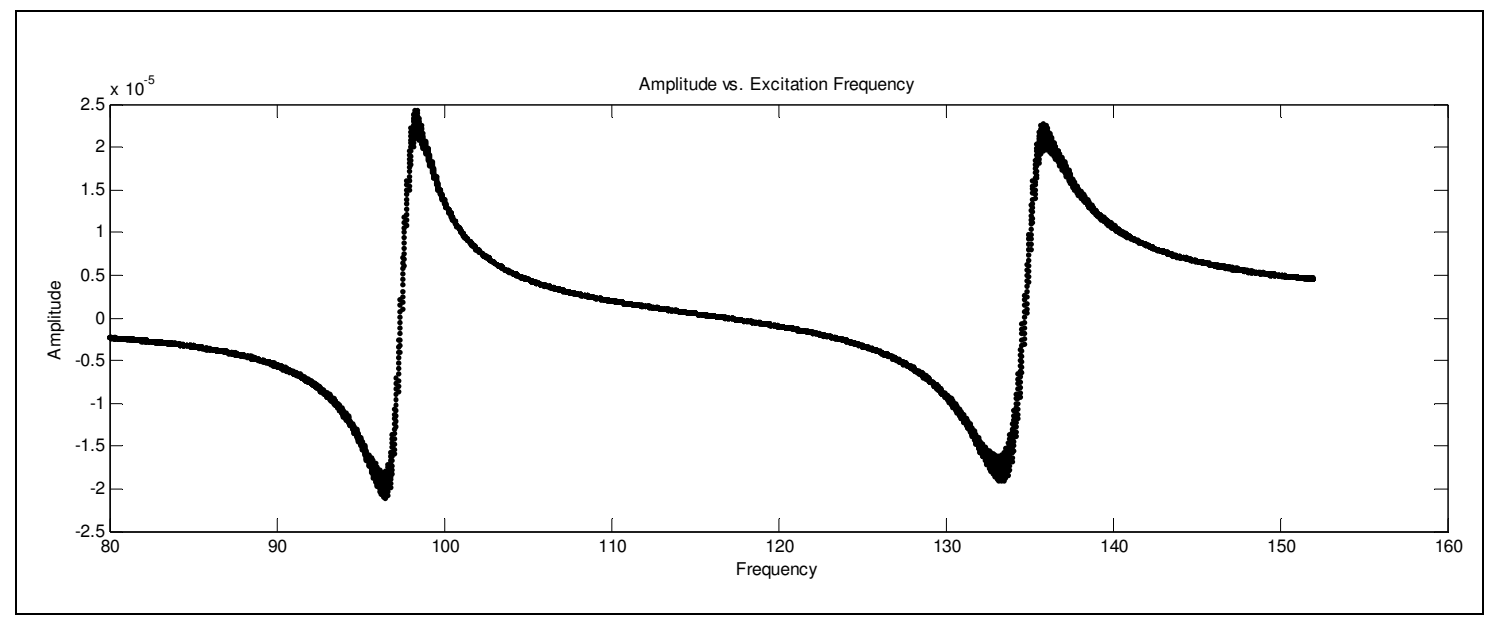

Fig. 45. Bifurcation Diagram with 4 Degree Misalignment with Finer Frequency Resolution for 80$150 \mathrm{~Hz}$ with $4^{\text {th }}$ Order Excitation Only.

The response clarifies that a normal single period response is apparent in the response of the system with a 4 degree misalignment through the two front driveshaft/ITD modes. The dynamic system was then subjected to the full forcing function with the multiple order excitations. The results for $4^{\text {th }}$ order excitation frequencies matching the previous analysis are presented in Fig. 46 . The same $4^{\text {th }}$ order period is used for the first return point calculation. 


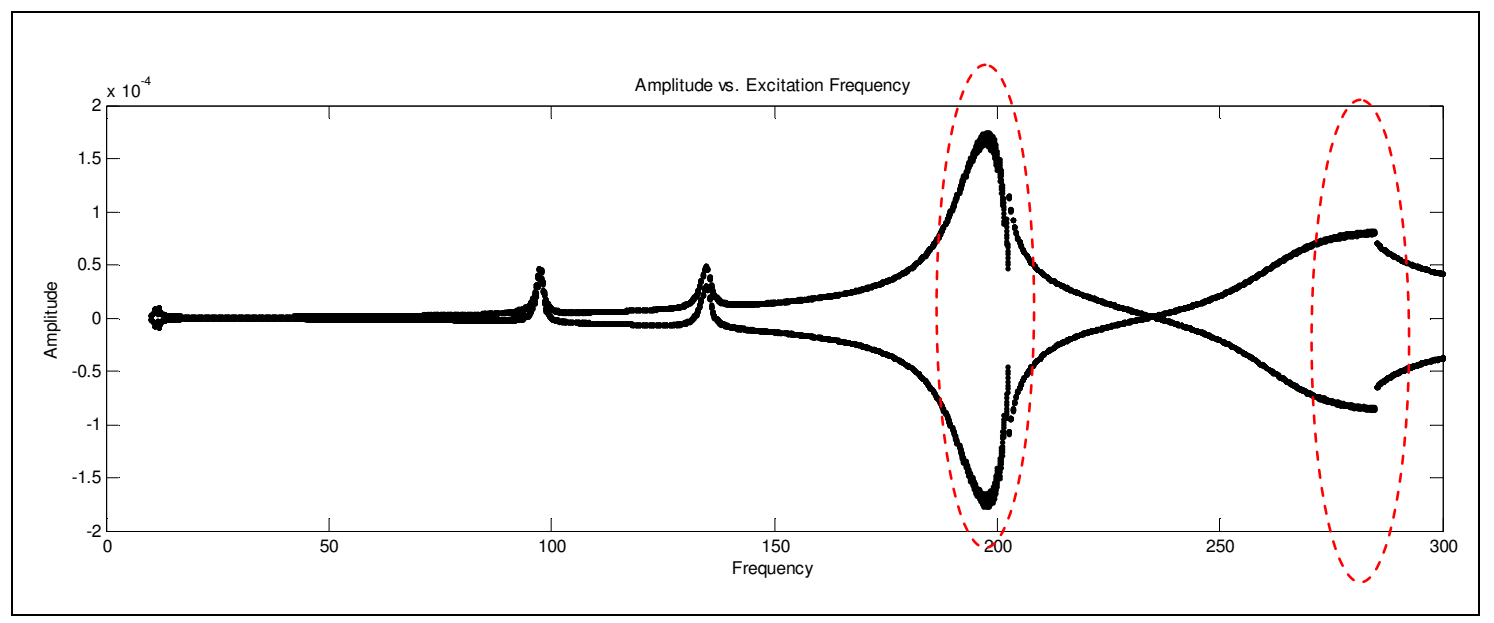

Fig. 46. Bifurcation Diagram with 4 Degree Misalignment Across 4x Input Frequencies of 2-250 $\mathrm{Hz}$ with Full Force Excitation.

The response with the full force equation shows the influence of the $2^{\text {nd }}$ order excitations as well as the $4^{\text {th }}$ order excitations through 2 points per frequency throughout the range of simulated frequencies. It is interesting to note that through $4^{\text {th }}$ order excitation until approximately $150 \mathrm{~Hz}$, the response was dominated by the $4^{\text {th }}$ order excitation. This is identified with both points being close together. Higher than $150 \mathrm{~Hz}$, the $2^{\text {nd }}$ order excitation from the full force excitation becomes dominant as it traverses the front driveshaft/ITD modes at just below 200 and $300 \mathrm{~Hz}$. There appears to be possible multiple solutions at 202 and $285 \mathrm{~Hz}$. A more detailed inspection of the $200 \mathrm{~Hz}$ response is presented in Fig. 47. 


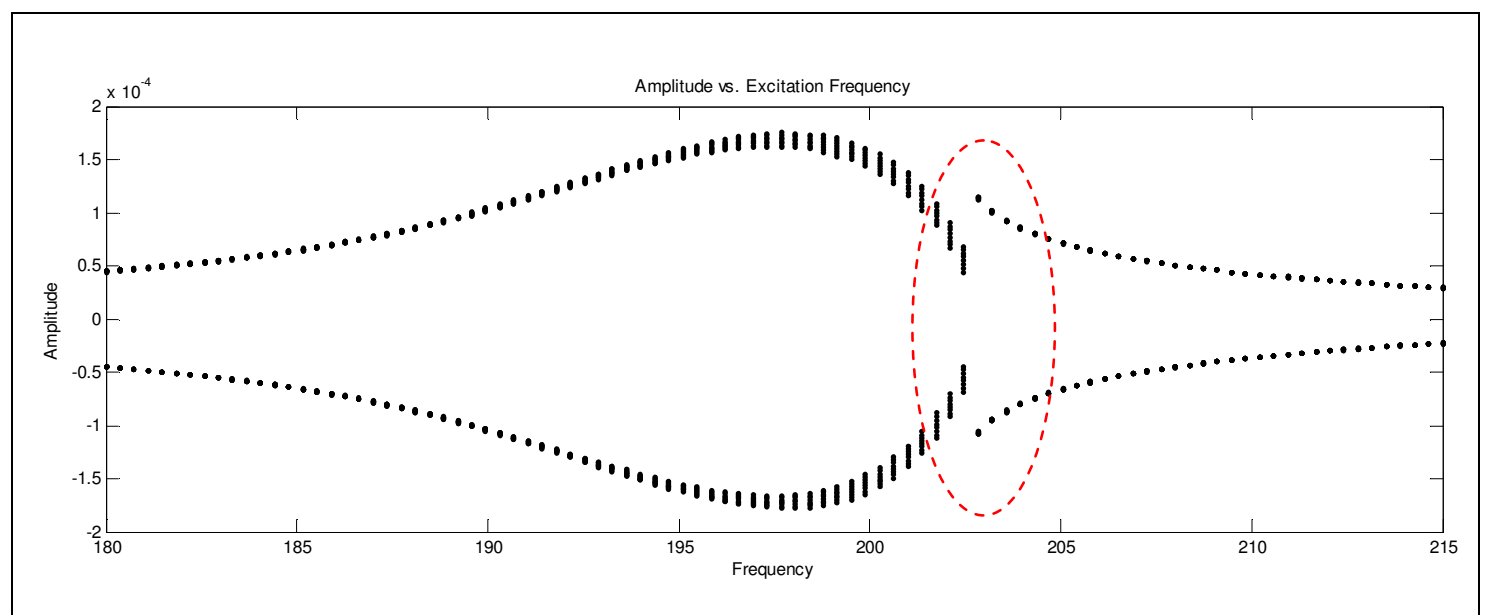

Fig. 47. Bifurcation Diagram with 4 Degree Misalignment with Finer Frequency Resolution for 80215 Hz with Full Force Excitation.

The Bifurcation diagram shows primarily 2 points per frequency. Considering possible calculation errors, there is basically 2 points per frequency identifying the $2^{\text {nd }}$ order response. The mirrored amplitudes of positive and negative amplitude certify the $2^{\text {nd }}$ order response is dominant. Even though there is no indication of multiple frequency content, there is a discontinuity at approximately $202 \mathrm{~Hz}$. This discontinuity indicates possible nonlinear behavior possibly leading to a nonlinear jump at this frequency. A similar response is noted at $285 \mathrm{~Hz}$, but has a jump down in place of a jump up. This aligns with the front driveshaft/ITD upper mode shape that created the nonlinear jump in the physical and simulation results as the $4^{\text {th }}$ order excited the natural frequency. The test system and simulation did not produce responses to $2^{\text {nd }}$ order excitation in this range, but from these results a similar non-linear jump would be predicted at this speed for the $2^{\text {nd }}$ order excitation.

For verification for any jump events, a finer resolution of the bifurcation diagram was calculated for frequencies around $130 \mathrm{~Hz}$. The results are plotted in Fig. 48. 


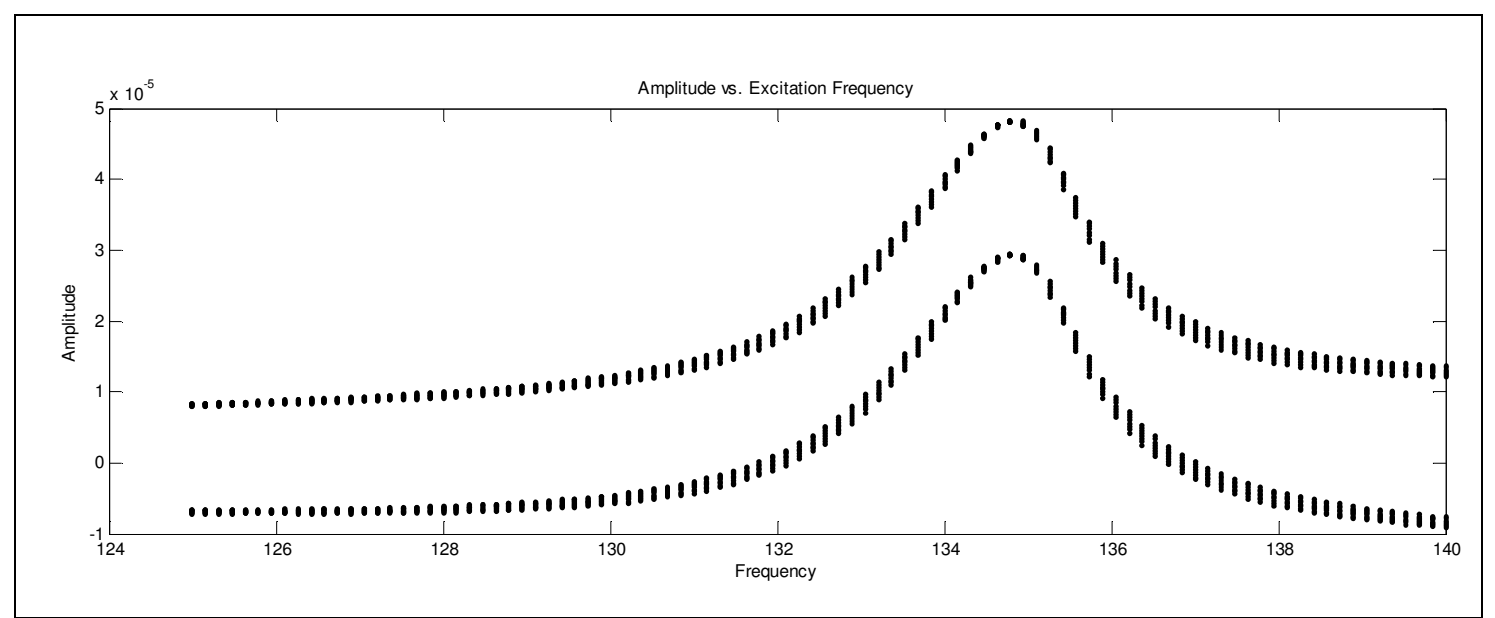

Fig. 48. Bifurcation Diagram with 4 Degree Misalignment with Finer Frequency Resolution for 124$140 \mathrm{~Hz}$ with Full Force Excitation.

The results show the response dominated by the $4^{\text {th }}$ order term, with a small $2^{\text {nd }}$ order contribution due to the full force excitation. But no similar discontinuity that was noted at $285 \mathrm{~Hz}$ wit the second order dominated excitation. The amount of nonlinearity as compared to the amplitude of excitation may not be enough to allow for a discrete identification from a simulation from a vector of initial conditions being all zero. Therefore, investigation into higher $4^{\text {th }}$ order excitation at around this frequency may reveal more effects of the nonlinearity with this analysis.

A similar approach to misalignment angle was calculated. This involved simulation of the system at multiple misalignment angles while maintaining a constant excitation frequency. This will help determine the effects of misalignment conditions. Therefore the response across misalignment angles from 0 to 23 degrees with the $4^{\text {th }}$ order excitation of $100 \mathrm{~Hz}$ only produced Fig. 49. This frequency was chosen as it is near the frequency of the front driveshaft ITD lower resonance. 


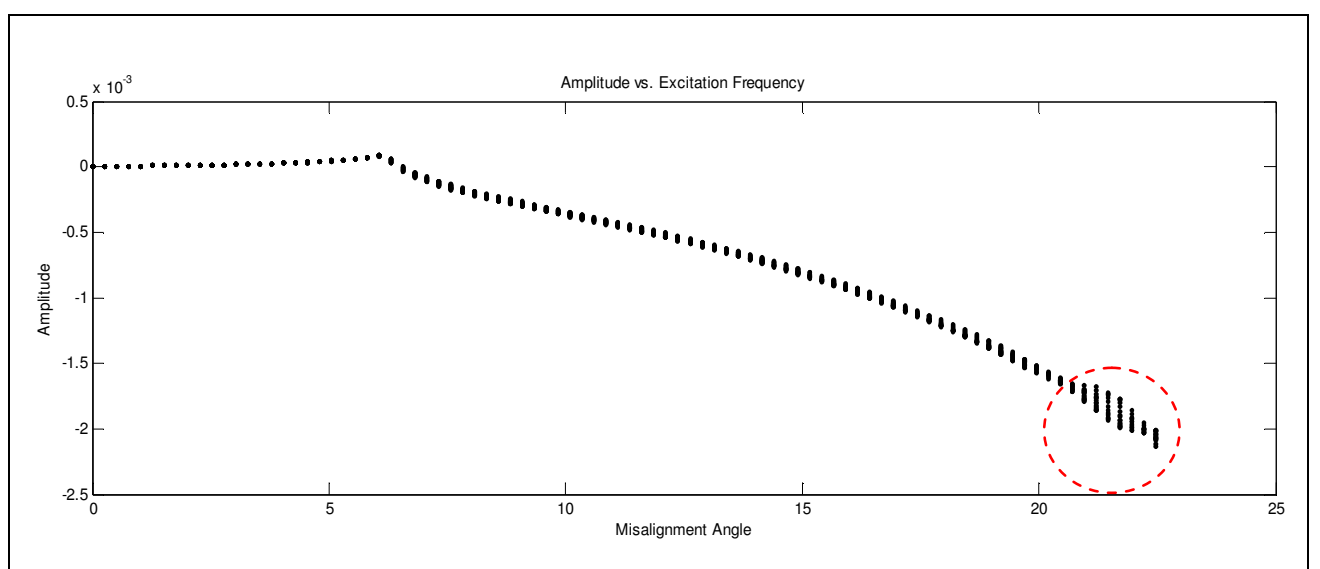

Fig. 49. Bifurcation Diagram Across Misalignment Angles of 0-23 Degrees with $4^{\text {th }}$ Order Excitation Only at $100 \mathrm{~Hz}$.

The results provided a fairly linear response across the misalignment angles. At above 20 degrees, there appears to be some minimal variation. The simulation qualified steady state when a maximum of a $2 \%$ error was calculated between 16 cycle periods. The analysis was stopped at 23 degrees because the response would not generate an error less than $8 \%$. This may be expected as this the front driveshaft/ITD lower mode did not produce a significant nonlinear response. Completion of the analysis at $135 \mathrm{~Hz}$, the second peak near the front driveshaft/ITD upper natural resonance may reveal more interesting results as it was the primary mode involved with the simulation and test nonlinear jumps. Therefore, misalignment angles from 0-25 degrees at $135 \mathrm{~Hz}$ are shown in Fig. 50. This frequency is at the peak of the steady state resonance response identified in the speed variation at 4 degrees previously, Fig. 48. 


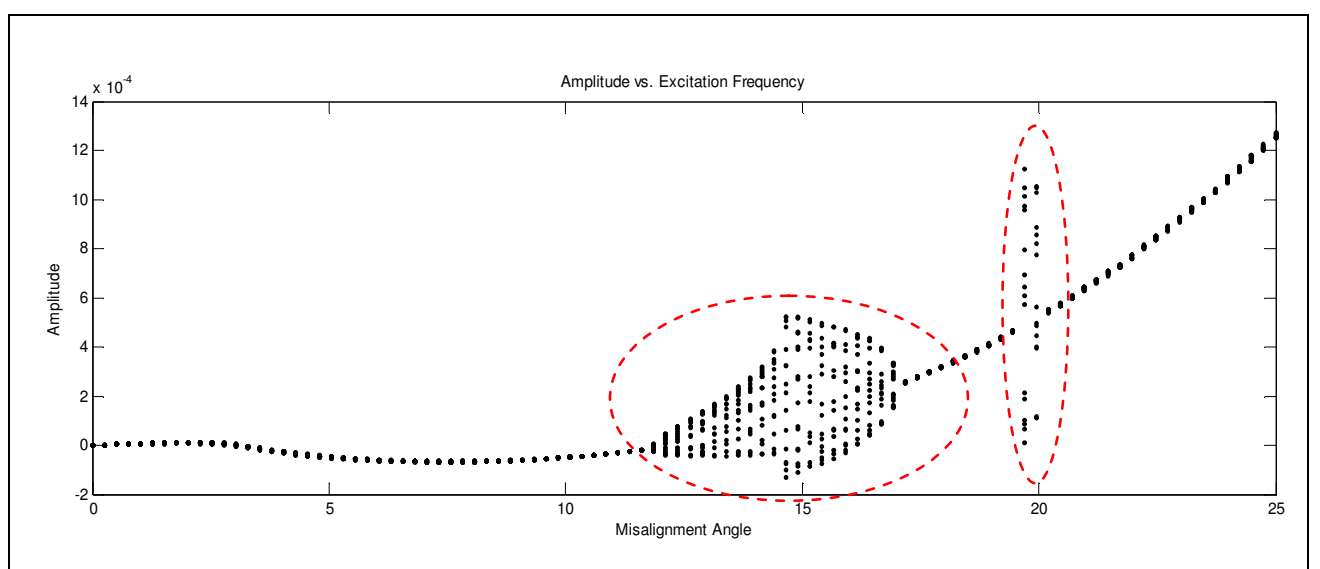

Fig. 50. Bifurcation Diagram Across Misalignment Angles of 0-23 Degrees with $4^{\text {th }}$ Order Excitation Only at $135 \mathrm{~Hz}$.

The misalignment angle variation at $135 \mathrm{~Hz}$ proved to produce a more interesting response showing some nonlinear behavior. Two areas of multiple frequency response are noted. The first from approximately 11.5 to 17 degrees of misalignment shows an increased number and range of multiple points of first returns. This indicates possible bifurcations and even possible chaotic vibrations. It is interesting that starting at approximately 11.5 degrees, the range grows until it reached approximately 14.5 degree where it has the maximum variation of amplitudes for the first returns. Increasing further produced decreasing variation of the first returns until it reaches a single point again at 17 degrees. The second range at approximately 19.5-20 degrees also shows multiple points of first returns, but the range does not grow and shrink like the first range of angles.

To define the angle where a bifurcation from a single point of first returns to a variation of first returns, finer resolution of misalignment angles was calculated. A first attempt on honing in on the bifurcation point produced Fig. 51. 


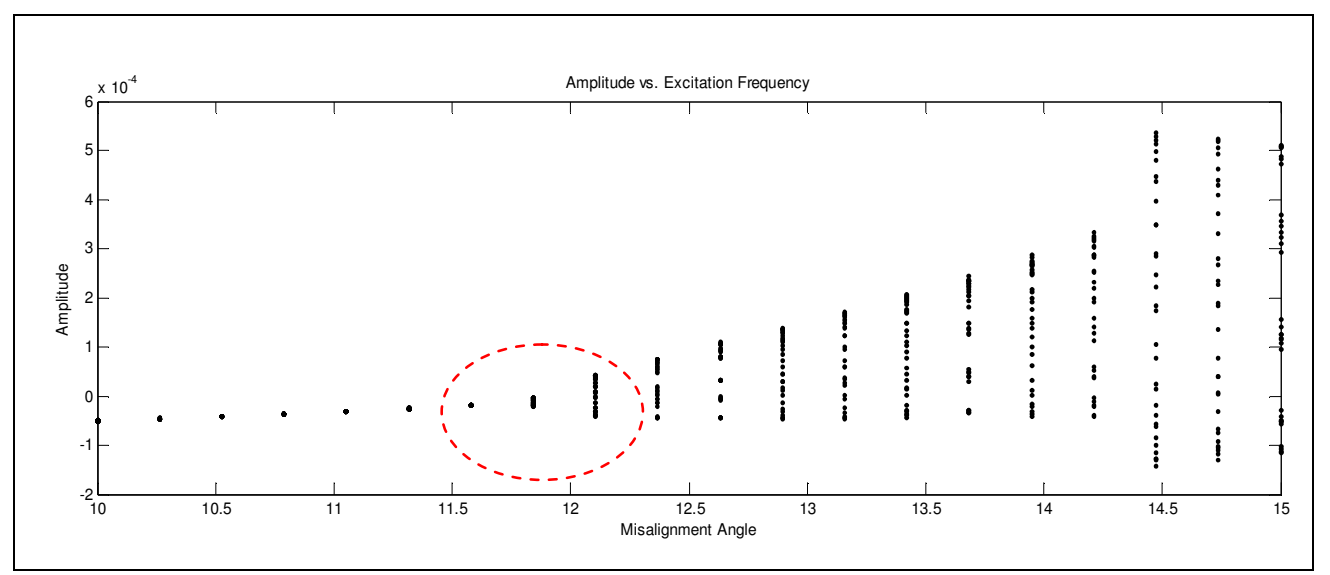

Fig. 51. Bifurcation Diagram Across Misalignment Angles of 10-15 Degrees with $4^{\text {th }}$ Order Excitation Only at $135 \mathrm{~Hz}$.

This closer look indicates the point of initial bifurcation at around 12 degrees. With this resolution is is not possible to identify the point of bifurcation, or if it is an orderly bifurcation. Therefore, a finer resolution was calculated and is plotted in Fig. 52.

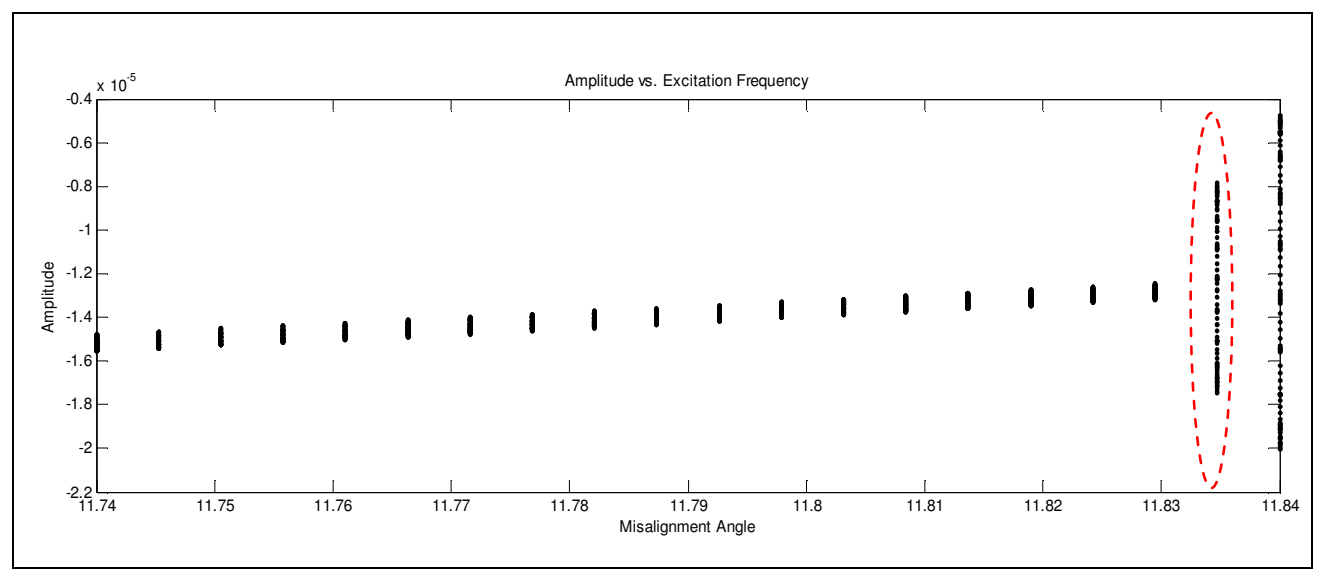

Fig. 52. Bifurcation Diagram Across Misalignment Angles of 11.74-11.84 Degrees with $4^{\text {th }}$ Order Excitation Only at $135 \mathrm{~Hz}$. 
The response isolates the initial point of bifurcation at 11.8325 degrees. The bifurcation does not indicate an orderly bifurcation such as a period doubling bifurcation. Additional evidence is acquired with a plot of the first returns on the phaseplane. The phase plane for 11.8325 degrees of misalignment is presented in Fig. 53 b).

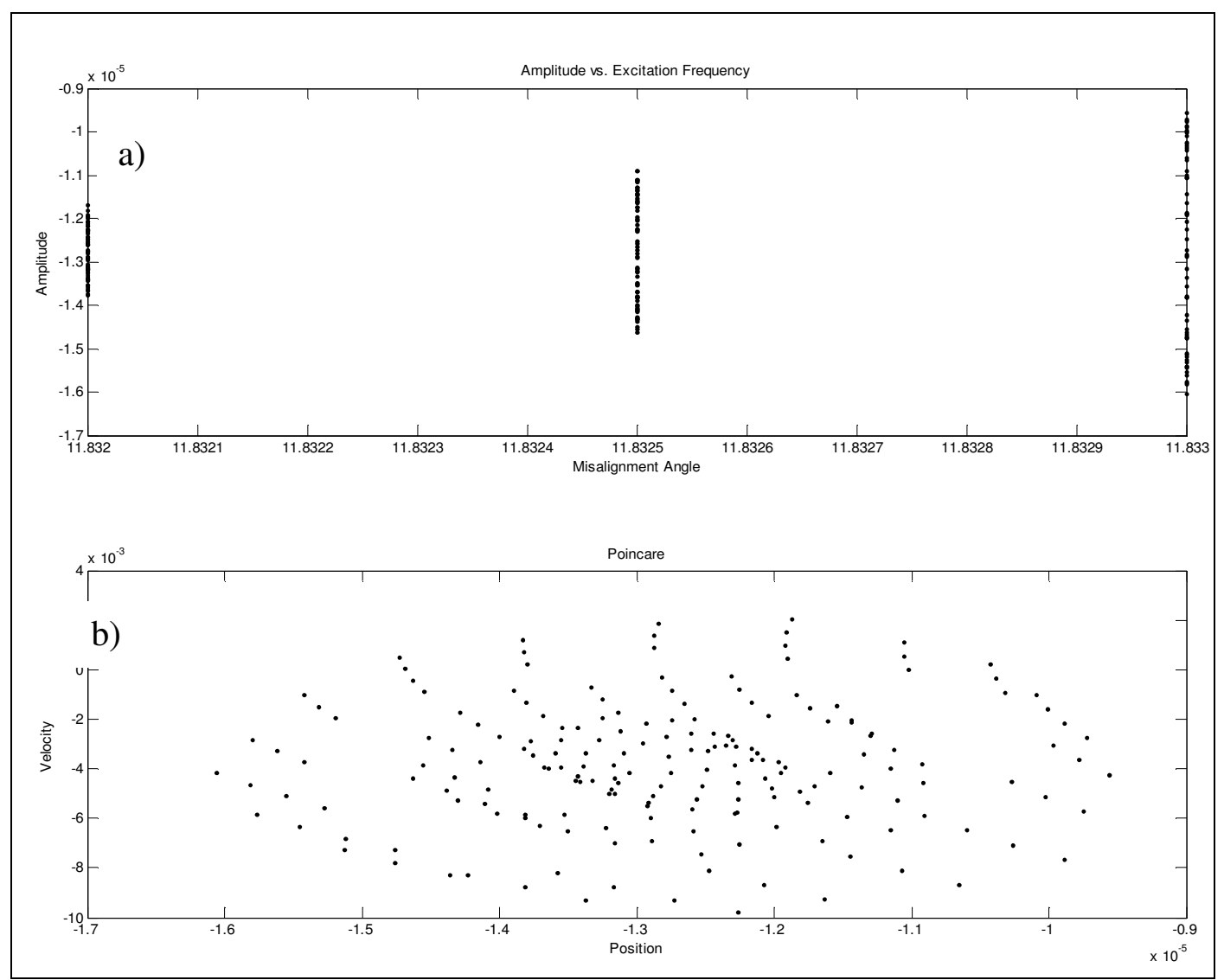

Fig. 53. Plot of First Returns on a) Bifurcation Diagram and b) Poincare Plot for 11.8325 Degrees of Misalignment with $4^{\text {th }}$ Order Excitation Only at $135 \mathrm{~Hz}$.

The Poincare plot does not show a coherent loop or multiple loop at this misalignment angle, and therefore it is unclear as to the nature of the bigurcation. To 
further investigate the bifurcation point, the time response of the front driveshaft at the ITD location is shown in Fig. 54.

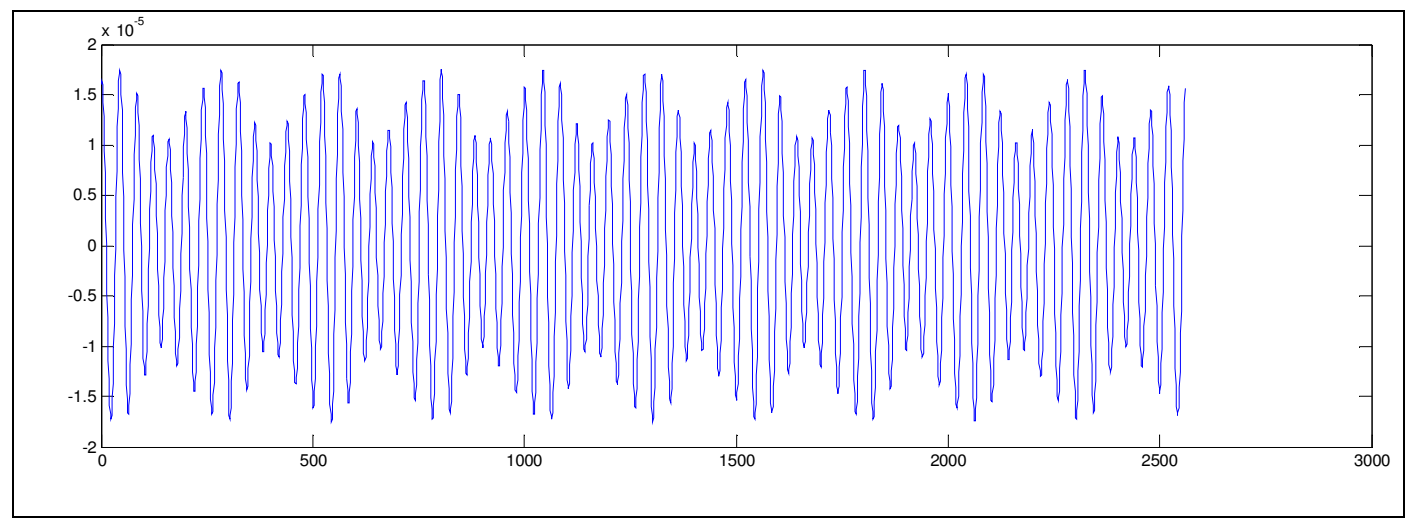

Fig. 54. Time Response of Front Driveshaft at ITD Location with 11.8325 Degrees of Misalignment Simulated at $135 \mathrm{~Hz}$.

The time response shows subharmoinic content, but it is unclear as to the frequency content of the subharmonic content. Subsequent spectral analysis will help reveal the subharmonic content. Prior to the spectral analysis, it is desired to understand more of the characteristics of the vibrations in this bifurcation region. Therefore, a similar analsis of 14.5 degrees of misalignment is completed. This misalignment condition showed the largest variation of first return amplitudes in the range of misalignment angles considered. An initial look of the 14.5 degree misalignment bifurcation diagram and phase-plane diagrams of the first returns are plotted in Fig. 55. 


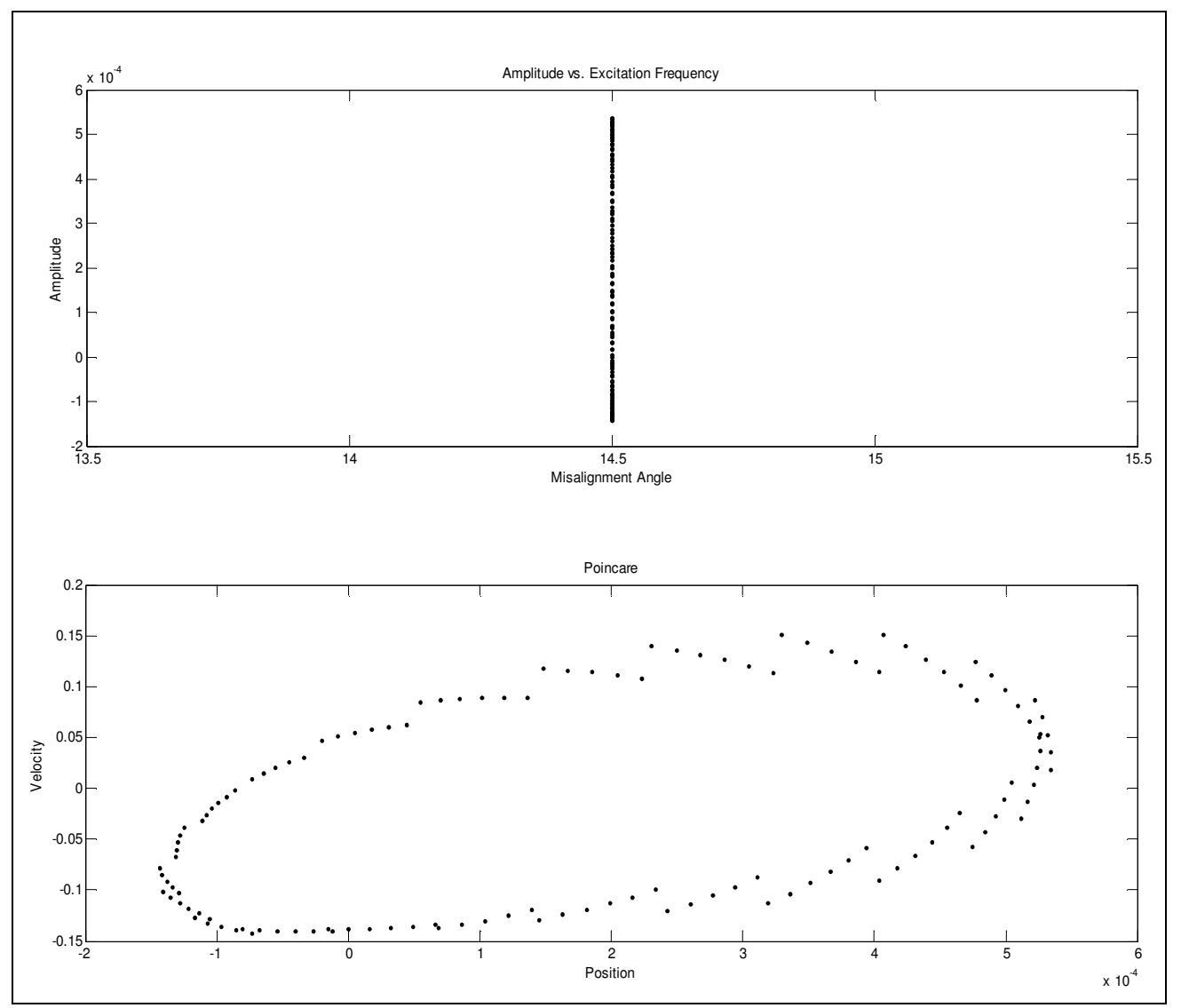

Fig. 55. Plot of First Returns on a) Bifurcation Diagram and b) Poincare Plot for 14.5 Degrees of Misalignment with $4^{\text {th }}$ Order Excitation Only at $135 \mathrm{~Hz}$.

The Poincare plot shows a possible closed loop indicating possible quasi-periodic or chaotic vibrations. Additional dat points are necessary for better understanding of the phase-plane results. A subsequent simulation obtaining the first return points for 320,256 cycles is plotted in Fig. 56. 


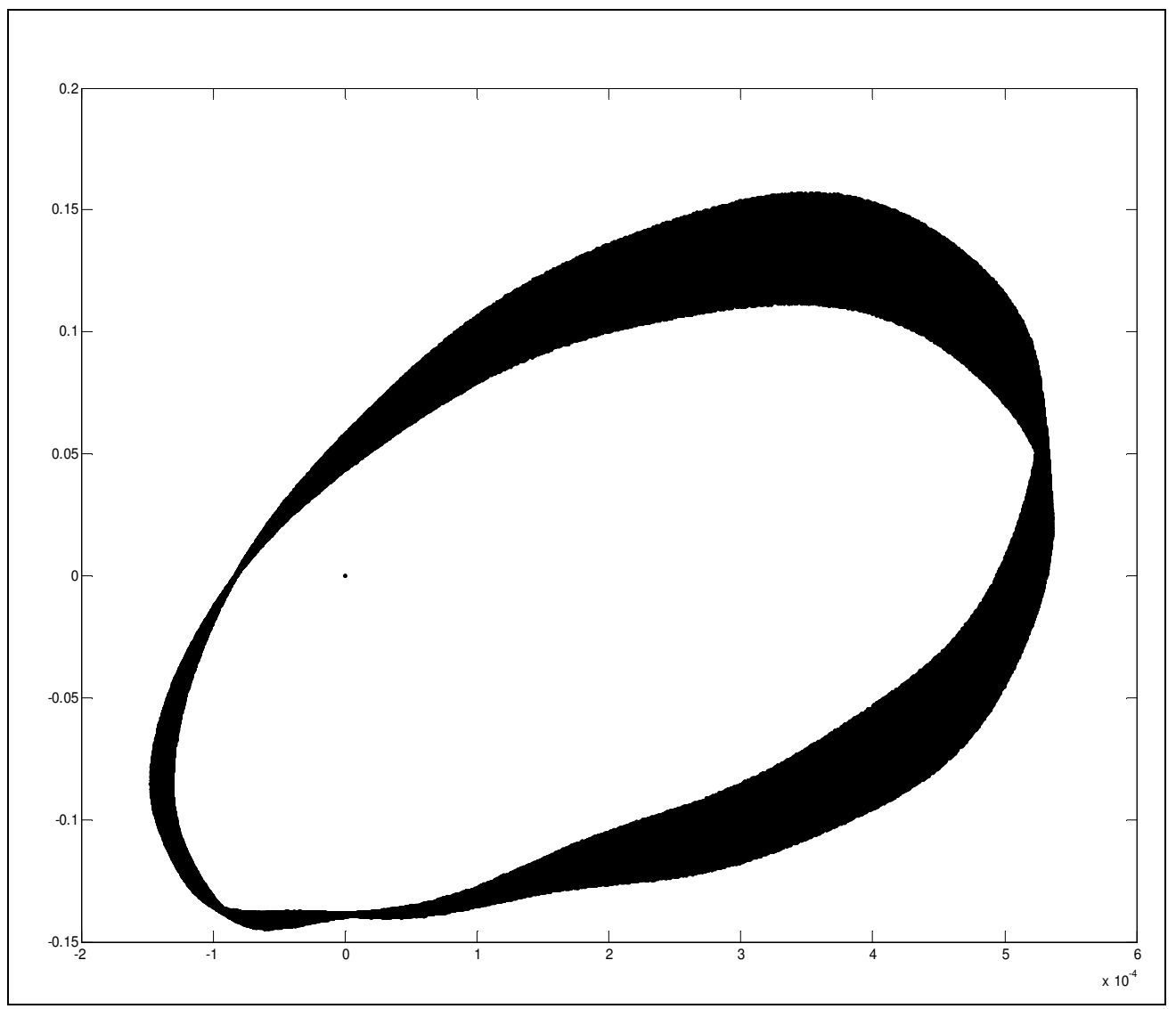

Fig. 56. Poincare Plot for 14.5 Degrees of Misalignment with $4^{\text {th }}$ Order Excitation Only at $135 \mathrm{~Hz}$ with 320,256 cycles.

The Poincare plot verifies a closed looped pattern. The pattern though is not elliptical, which would clearly identify quasi-periodic vibrations. Therefore, further analysis into identifying chaotic vibrations is necessary to understand these vibrations. In addition, a simulation with the full force equation was also completed to understand if the other force vibration orders would significantly influence the response of the system at this misalignment and excitation frequency state. The response is plotted in Fig. 57. 
109

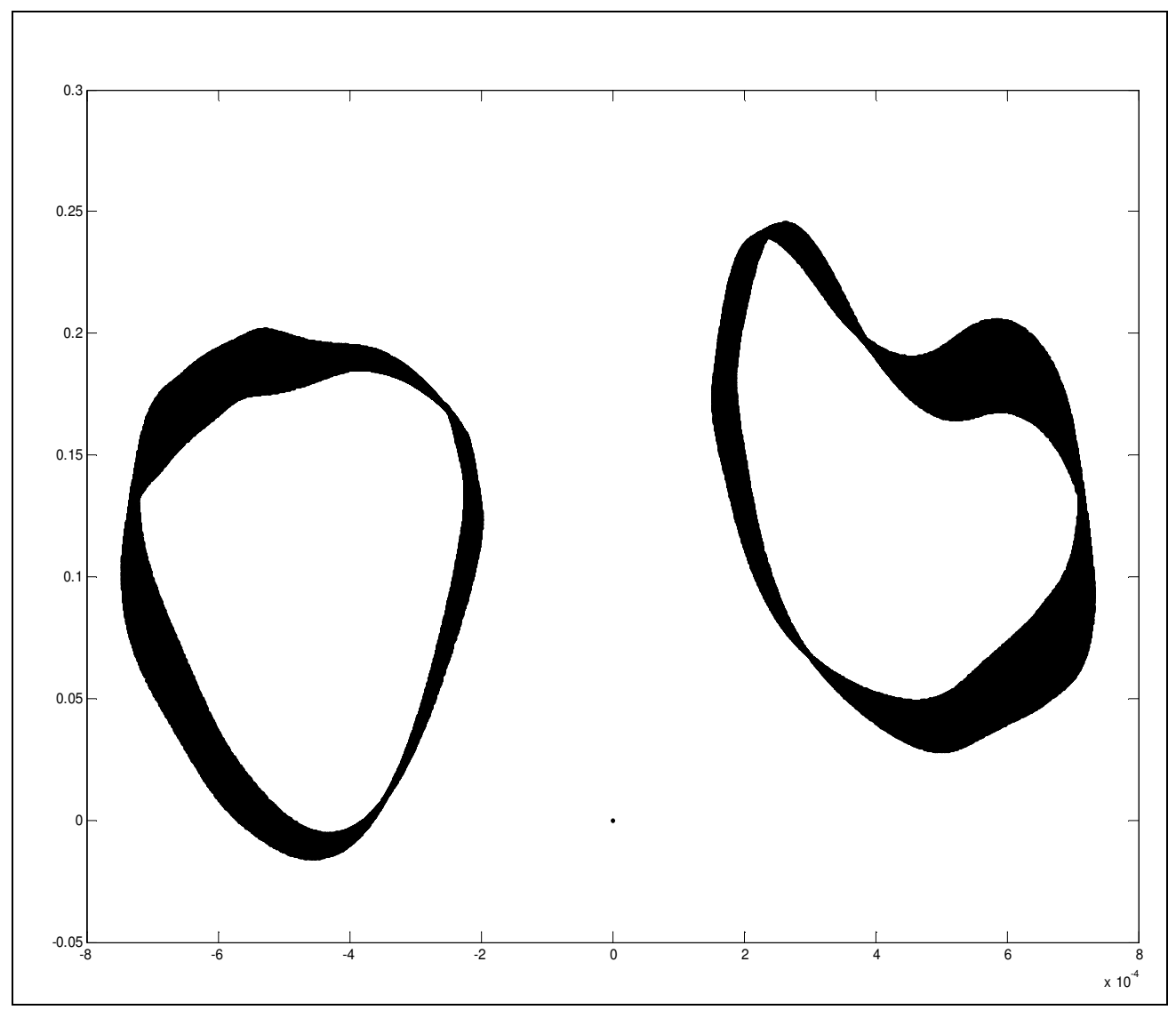

Fig. 57. Poincare Plot for 14.5 Degrees of Misalignment Full Moment at $135 \mathrm{~Hz}$ with 320,256 cycles.

The response clearly shows two separate closed loops. This is once again an effect of the addition of the $2^{\text {nd }}$ order excitation. It is interesting to note that the two closed loops are once again not only non-elliptic, but are different for the two loops. This once again gives the indication of possible chaotic vibrations at this condition. The plot of the physical response at the front driveshaft at the ITD connection node is pictured in Fig. 58. 


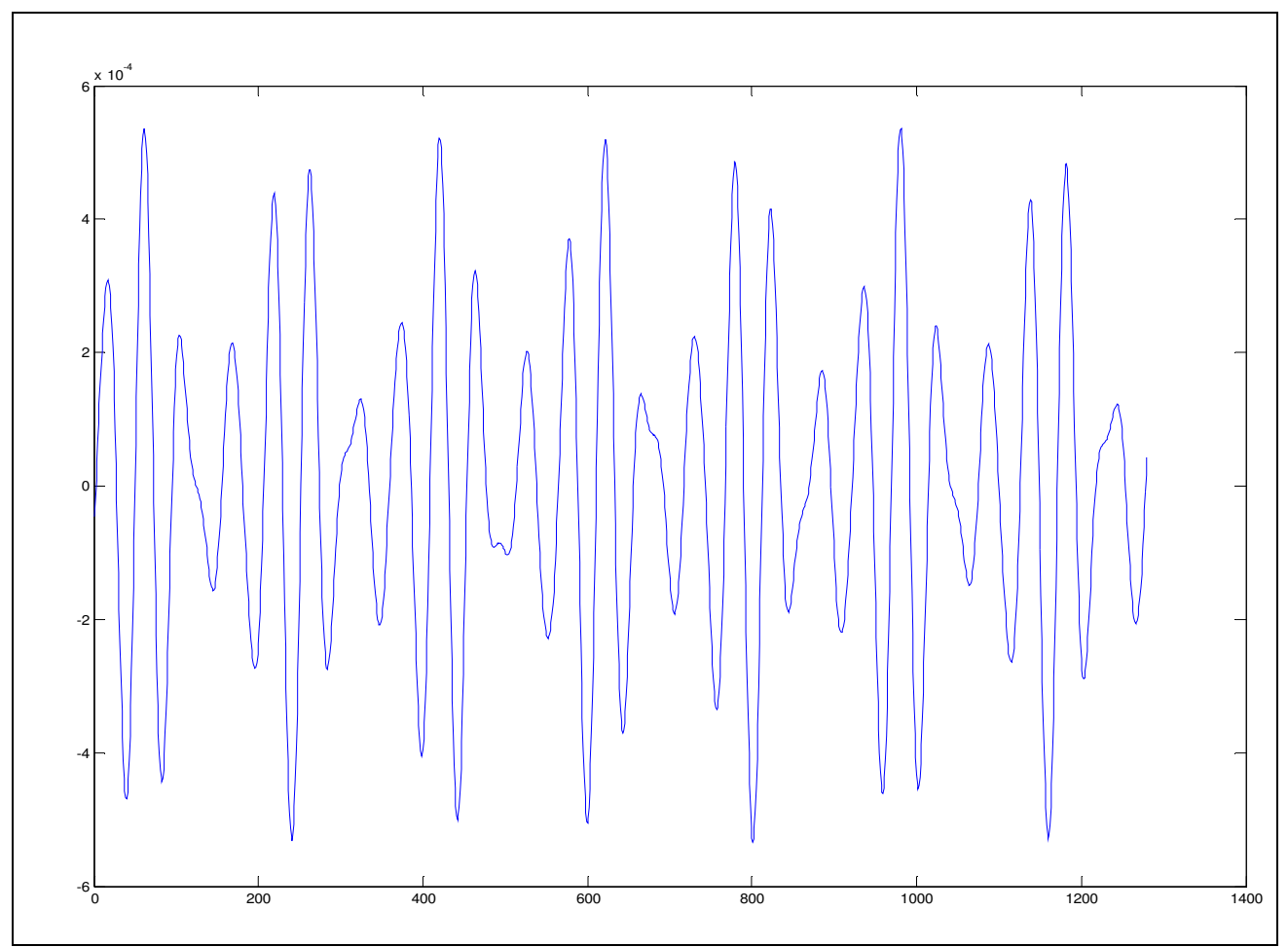

Fig. 58. Time Response of Front Driveshaft at ITD Location with 14.5 Degrees of Misalignment Simulated at $135 \mathrm{~Hz}$.

The time response shows significant variation of the amplitude response. In addition, there is significant variation and no cohesive pattern is noted. Therefore, subsequent spectral analysis is necessary to understand the frequency content of the presented time signals. In addition, subsequent analysis clarifying if the observed nonlinear vibrations are chaotic is also necessary. 


\section{Spectral Analysis}

The previously presented time waveforms were sufficiently complicated that identification of the multiple frequency content was not easily discernable. This situation is commonly experienced with measured data. Fast Fourier Transformation, FFT, techniques are used to calculate the frequency content of the time signal. Due to the nature of the simulated time signals, the same techniques may be utilized to determine the frequency content of the complex simulated time waveforms.

An FFT was calculated for the time waveforms from the simulation providing the First returns points in the bifurcation and Poincare analysis. A contour plot of the frequency spectrums at each misalignment angle to $2000 \mathrm{~Hz}$ is presented in Fig. 59.

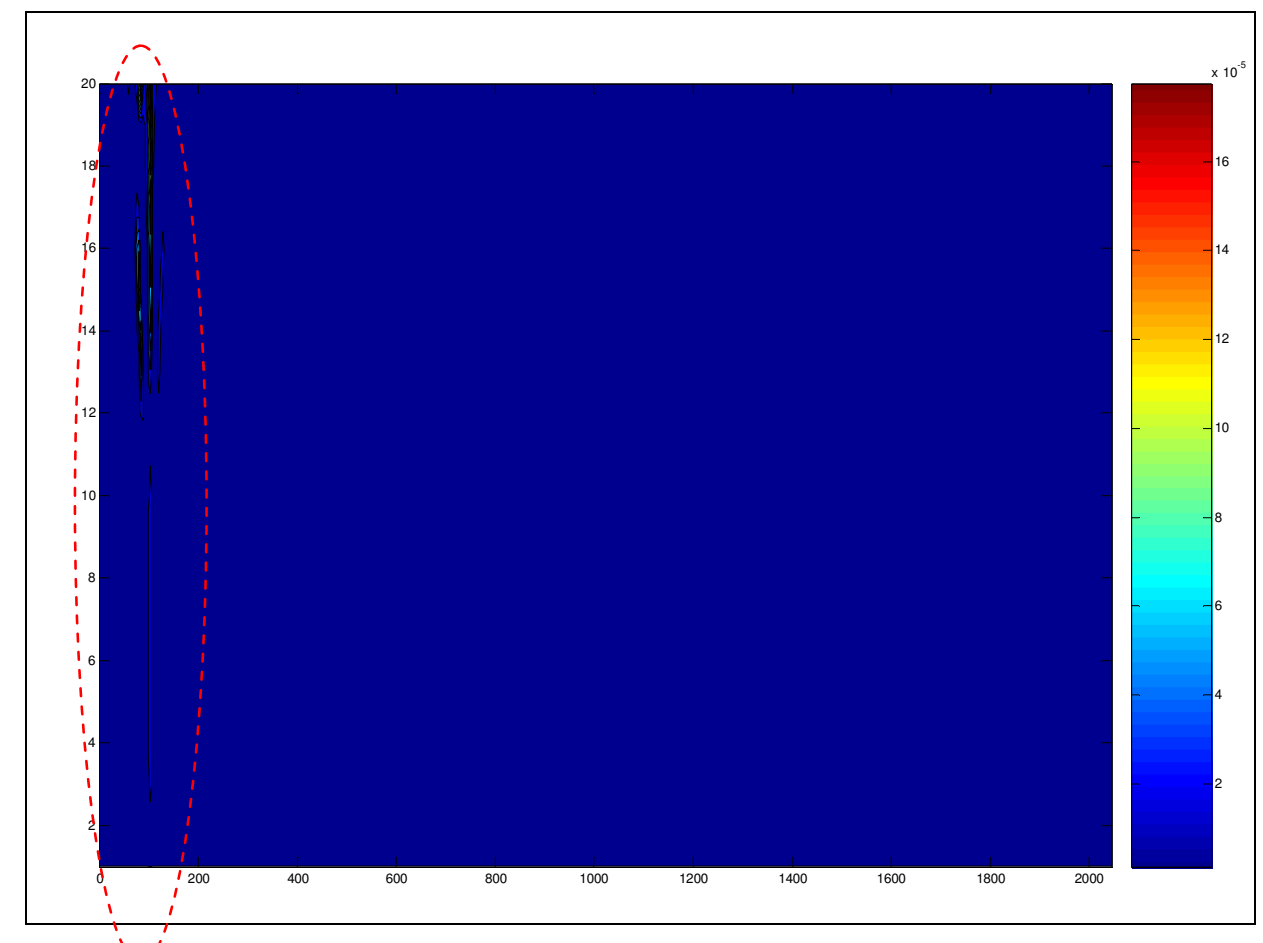

Fig. 59. Contour Plot of Frequency Spectrums at each Misalignment Angle from 0-20 Degrees at 135 Hz, 4x Excitation. 
The spectrum reveals a single frequency response from 0 to approximately 11.8 degree. There are multiple frequencies around the excitation frequency at misalignment angles 11.8-17degrees, and again just below 20 degrees. The frequency spectrum shows agreement with the bifurcation diagram that there are multiple frequencies these misalignment angle ranges. A closer inspection of the misalignment angles of interest from 12-15 degrees is presented in Fig. 60.

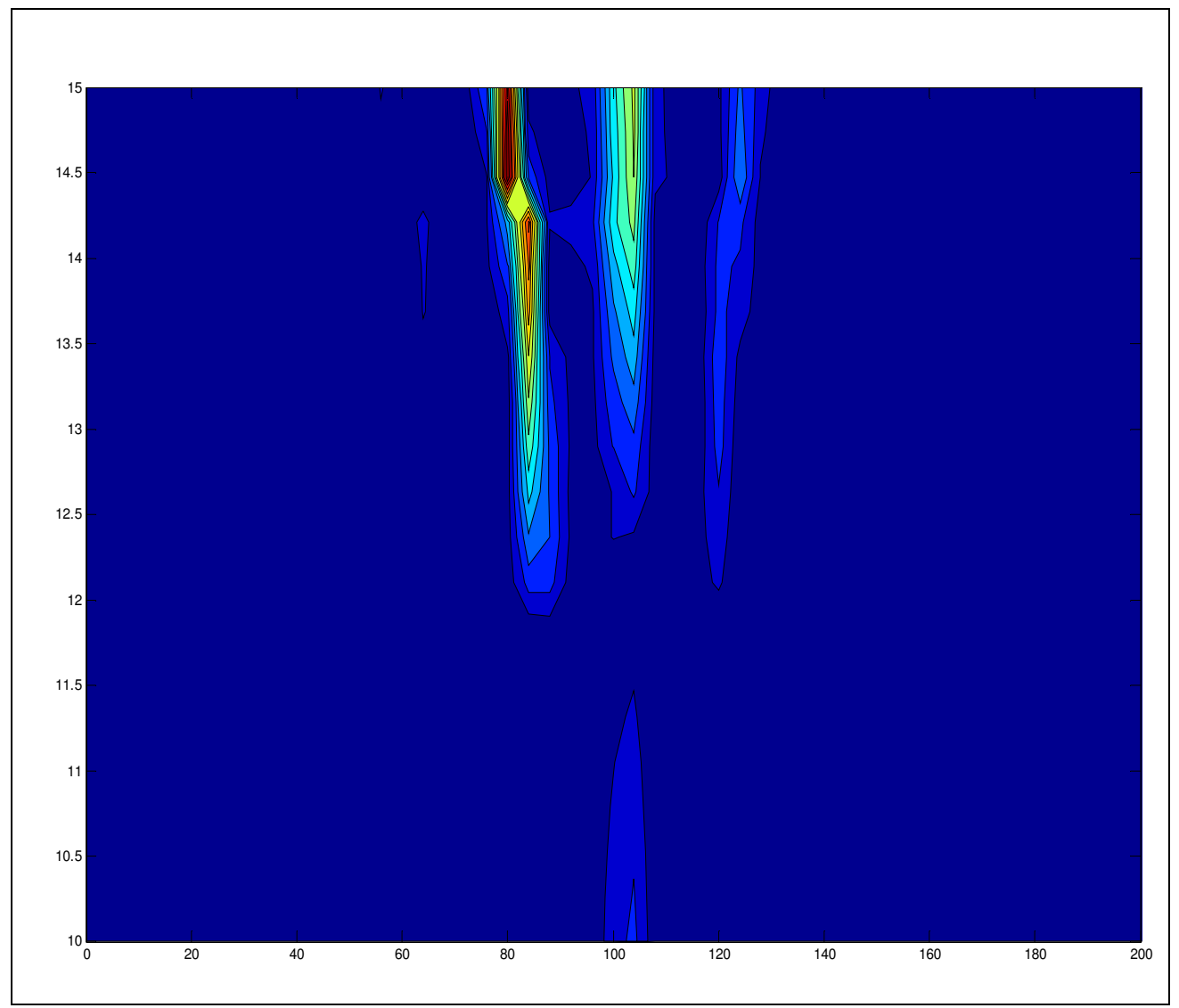

Fig. 60. Contour Plot of Frequency Spectrums to $200 \mathrm{~Hz}$ at each Misalignment Angle from 10-15 Degrees at $135 \mathrm{~Hz} 4 x$ Excitation. 
The spectrum shows that multiple frequencies appear at approximately 11.8 degrees. This is once again in agreement with the bifurcation and Poincare analysis. The multiple frequencies vary in frequency with misalignment angle. It is interesting to note that two frequencies appear to be mirrored around the excitation frequency. At around 14 degrees, an additional $4^{\text {th }}$ frequency becomes significant enough to become noticeable in the contour plot. A section at 14.5 degrees showing the spectral content to $200 \mathrm{~Hz}$ is presented in Fig. 61.

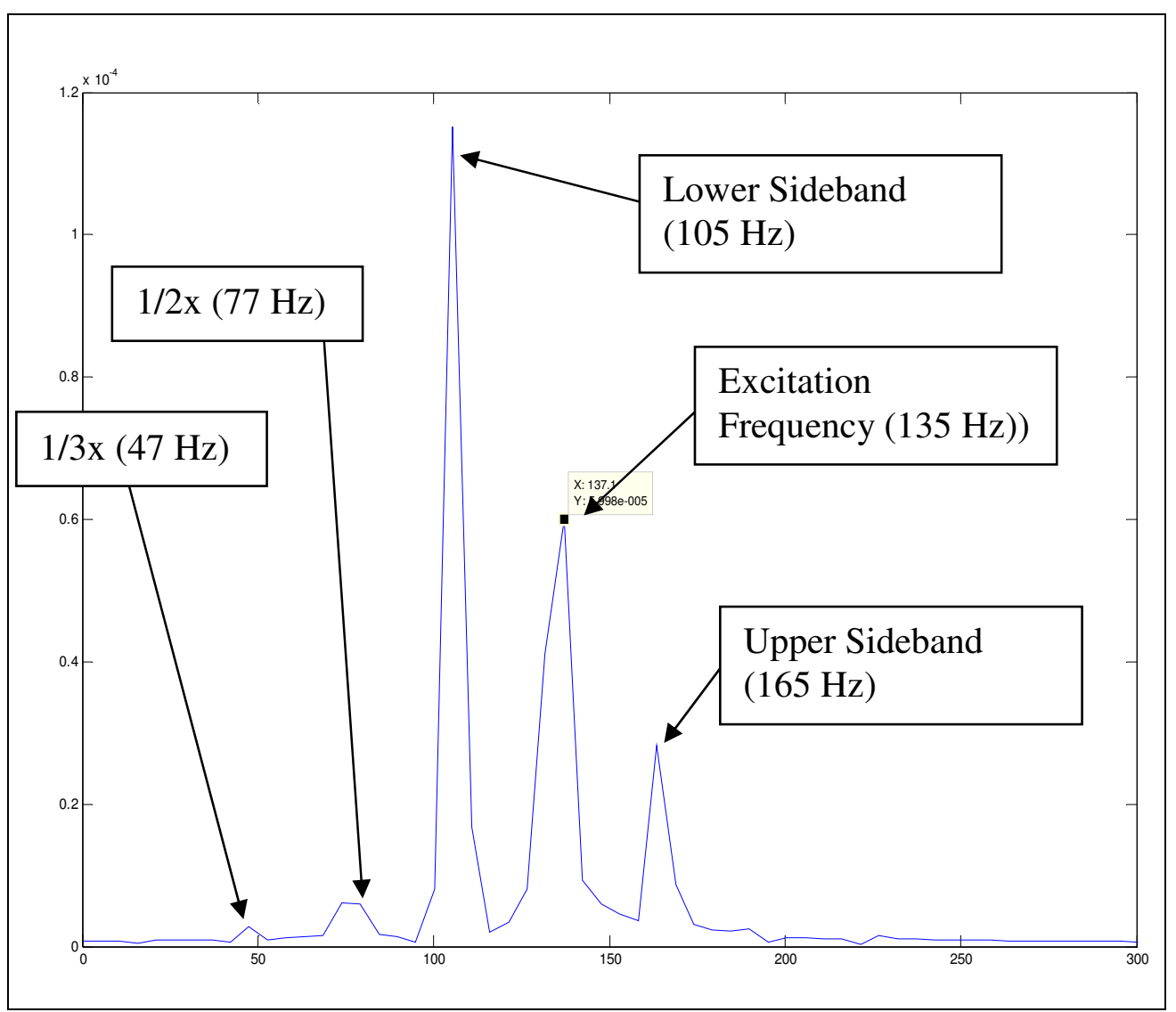

Fig. 61. Frequency Spectrum to $200 \mathrm{~Hz}$ at 14.5 Degrees of Misalignment from $1000 \mathrm{~Hz}$ Sampling Rate and Blocksize of $\mathbf{1 0 2 4}$ Samples. 
Five distinct peaks are notable on the linear amplitude plot. This includes the excitation frequency is at $135 \mathrm{~Hz}$, two sidebands, and two sub-harmonics. Of the sidebands, the large peak is at $105 \mathrm{~Hz}$ below the excitation frequency, and is at the front driveshaft/ITD lower resonance. In addition, an upper sideband at $165 \mathrm{~Hz}$ is at the same frequency spacing at this condition as the two front driveshaft/ITD modes.. The subharmonics appear at approximately $77 \mathrm{~Hz}$ and $47 \mathrm{~Hz}$. This is at approximately the 1/2 and $1 / 3^{\text {rd }}$ of the excitation frequency, respectively. A longer simulation period would provide more points to get better resolution and more averaging on the calculation of the spectrum. A subsequent plot with a larger sampling period is presented in Fig. 62.

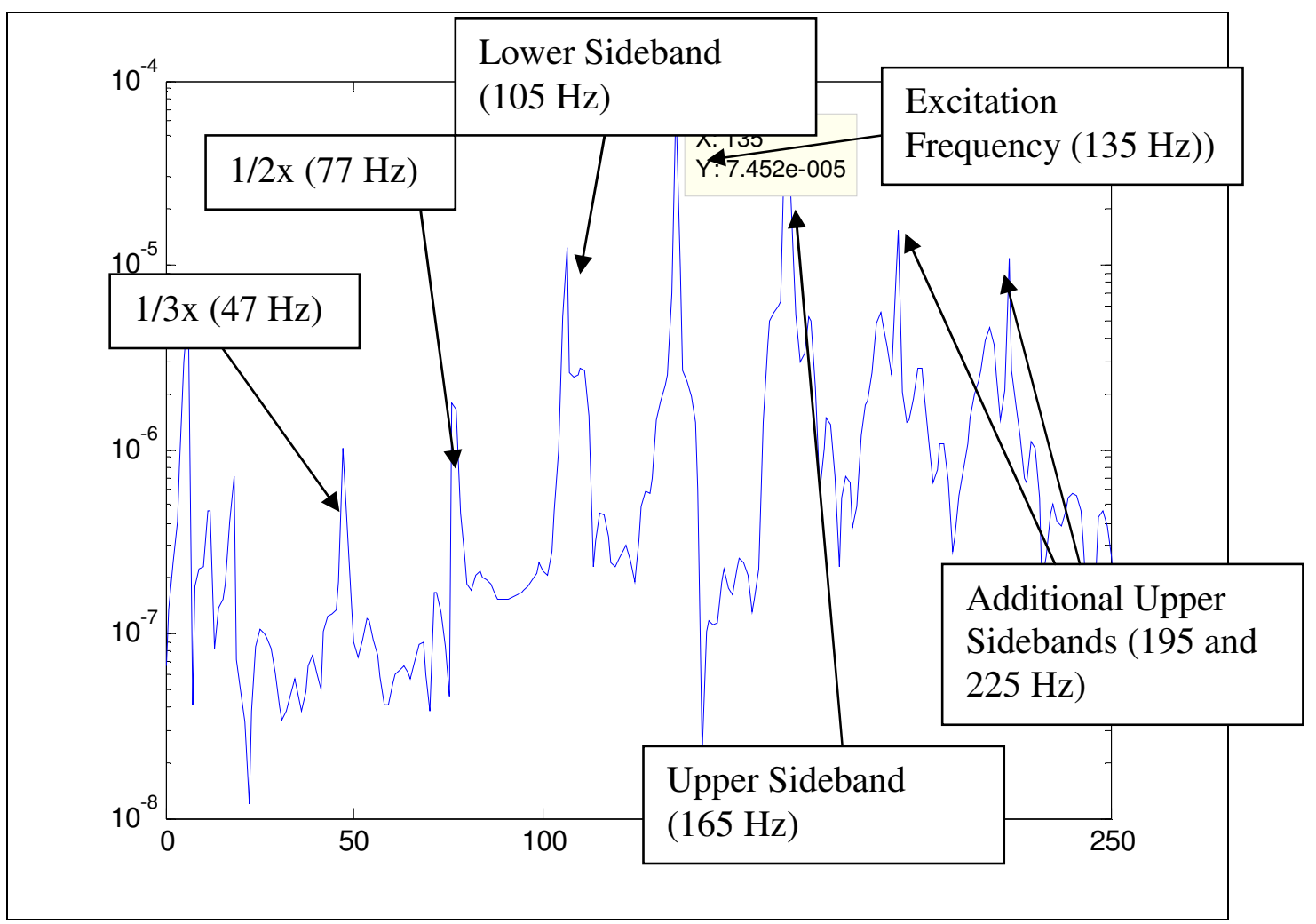

Fig. 62. Frequency Spectrum to $200 \mathrm{~Hz}$ at 14.5 Degrees of Misalignment from $1000 \mathrm{~Hz}$ Sampling Rate and Blocksize of 4096 Samples on a Log Scale. 
The longer simulation period not only increased the resolution, but also clarified some higher harmonic terms. This includes additional superharmonic sidebands at the multiples of the separation frequency of approximately $30 \mathrm{~Hz}$ of the first sideband. The multiple frequencies are a result of the non-linearity and are an indication of chaotic vibrations. As an additional simulation was completed to allow for additional time simulation, some trasient effects are seen with the addition of the response of the 16.5 $\mathrm{Hz}$ centerbearing bouce mode at the lower end of the spectrum. This is not an included nonlinear effect of the system. As a comparison, the frequency spectrum at 1 degree of misalignment is presented in Fig. 63.

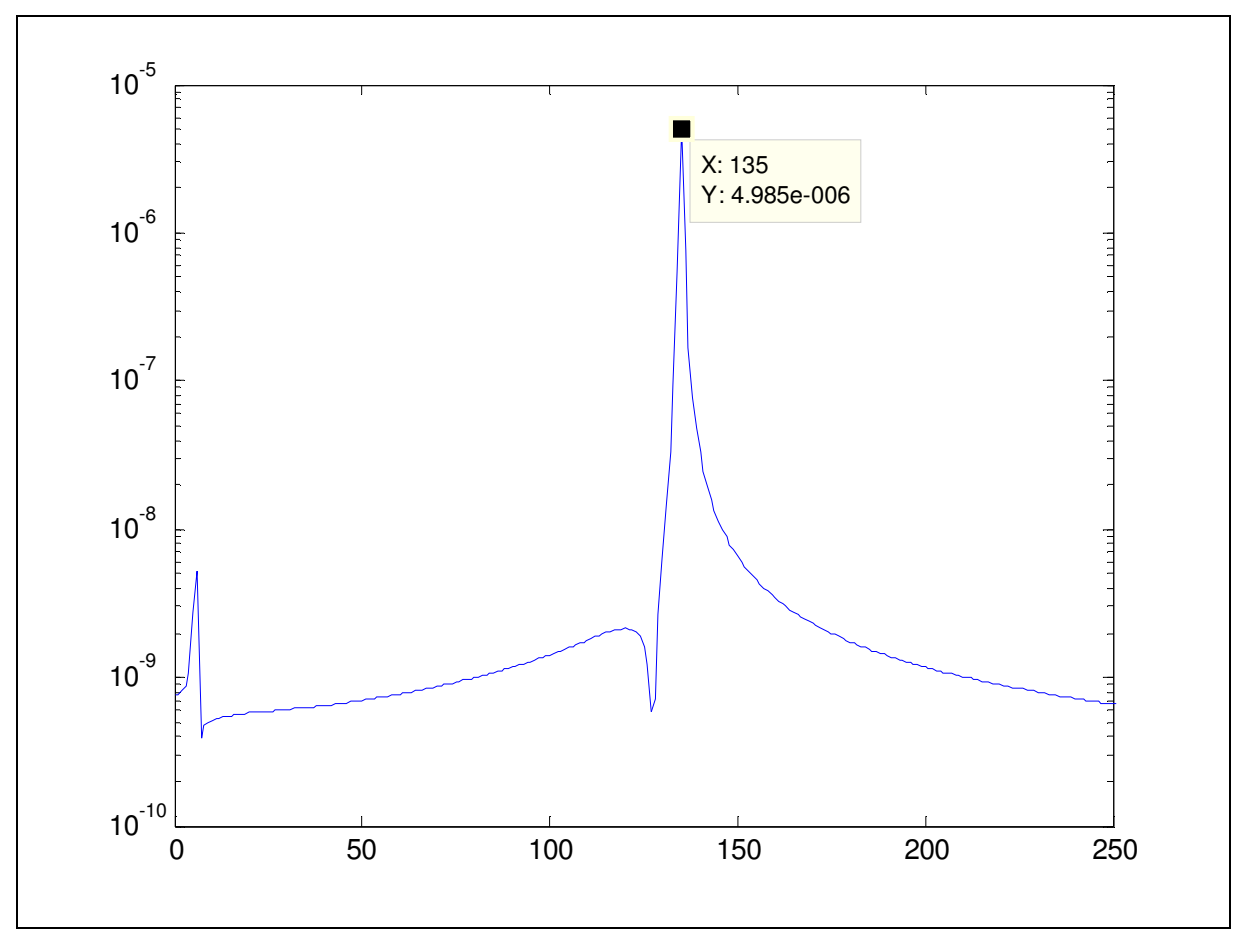

Fig. 63. Frequency Spectrum of Simulation Results with 1 Degree Misalignment. 
The frequency spectrum of the 1 degree of misalignment shows clearly the response only at the excitation frequency. Once again, since this was an additional simulation, a peak at the center bearing bounce mode is noted at $16.5 \mathrm{~Hz}$ as this mode was not yet settled. Therefore this additional peak is not part of the nonlinear response of the system.

Spectral analysis helped to understand the multiple frequency components of the time response at the various angles. The analysis identified both sideband type frequencies with peaks separated equal frequency spans apart from the excitation frequency. It also identified $1 / 2$ and $1 / 3$ rd sub-harmonics and additional super harmonic terms at additional sideband frequency spans above the excitation frequency at 14.5 degrees of misalignment. Similar results proved no nonlinear multiple frequency response at 1 degrees of misalignment. Therefore, the frequency spectrums clearly identify nonlinear response effects indicating possible chaotic vibrations in the various misalignment angles. Additional analysis is necessary to confirm these vibrations as chaotic.

\section{Lyapunov}

Determination of whether a system exhibits chaotic vibrations can be completed by calculating the Lyapunov Exponents. As will all nonlinear systems, there is a significant dependence on initial conditions. A unique feature of chaotic systems is that through small variations in the phase space, the results move exponentially away from each other through time. From this exponential nature, the exponents quantifying the 
rate of exponential deviations can be calculated and are called the Lyapunov Exponents. From a system of equations, the Lyapunov Exponent is defined as $\lambda$ in

$$
\lambda_{i}=\lim _{t \rightarrow \infty} \frac{1}{t} \log _{n} \frac{p_{i}(t)}{p_{i}(0)}[46]
$$

where $p_{i}$ are the length of the path along an n-ellipsoid defined by the vibrations, and $t$ is time. The log base $\mathrm{n}$ is subjective, and can be chosen as any base. Background literature has typically used the natural $\log$, ln, or base 2. For a system of equations, there are equivalent numbers of Lyapunov exponents are there are degrees of freedom in a system of $1^{\text {st }}$ order differential equations. Therefore, a spectrum of Lyapunov Exponents is created for a system.

Methods of calculation of the spectrum have been outlined in previous work[45, 46]. These methods use an iterative approach to test for the value of the Lyapunov coefficients, and converge on a solution after many repetitions. For each iteration, a set of orthogonal initial input vectors are applied to the system and simulated simultaneously with the nonlinear set of differential equations, and through a set of linearized differential equations. The linearized set of differential equations is updated at each simulation time interval. A secondary periodic interval is used in calculating the Lyapunov exponent. For every subsequent Lyapunov calculation period, the vectors are once again orthonormalized.

The final Lyapunov exponents are calculated as the solution of the time based average converges to a solution in 


$$
\lambda_{i}=\frac{1}{t_{M}-t_{0}} \sum_{k=1}^{M} \log _{n} \frac{L^{\prime}\left(t_{k}\right)}{L\left(t_{k-1}\right)},
$$

where $M$ is the total number of calculated Lyapunov exponent calculation periods, $L^{\prime}$ is Length of the final state vector, and $L$ is the initial state at the each of the Lyapunov exponent calculation periods. A more detailed explanation of the method of calculating the Lyapunov Exponents is included in Appendix A. In addition, Lyapunov exponents are calculated for various settings of the Duffing, Lorenz, and Rossler-chaos equations and compared to known results.

From the calculation, it can be seen that if the ratio of $L^{\prime}$ to $L$ is greater than 1 , representing a system with exponential growth, then the exponent will be positive. If the ratio is less than 1, representing a system with exponential decay, the exponent will be negative. If the ratio is equal to one, the Lyapunov exponent will be zero. This point creates the border, and is documented that any continuous time dependant system will have at least one zero exponent[46]. Therefore, the Lyapunov exponents quantifies the system as chaotic (positive exponent) or nonchaotic (zero or less exponent). In addition, the maximum exponent of a system with multiple degrees of freedom can have chaotic vibrations determined by the sign of the maximum Lyapunov exponent.

Applying the technique to calculate the Lyapunov exponents to the driveshaft system reveals the basic state of the system. The Lyapunov exponents were calculated for the system with 14.5 degrees of misalignment at $135 \mathrm{~Hz}$ as previously presented, and the results are plotted in Fig. 64. 


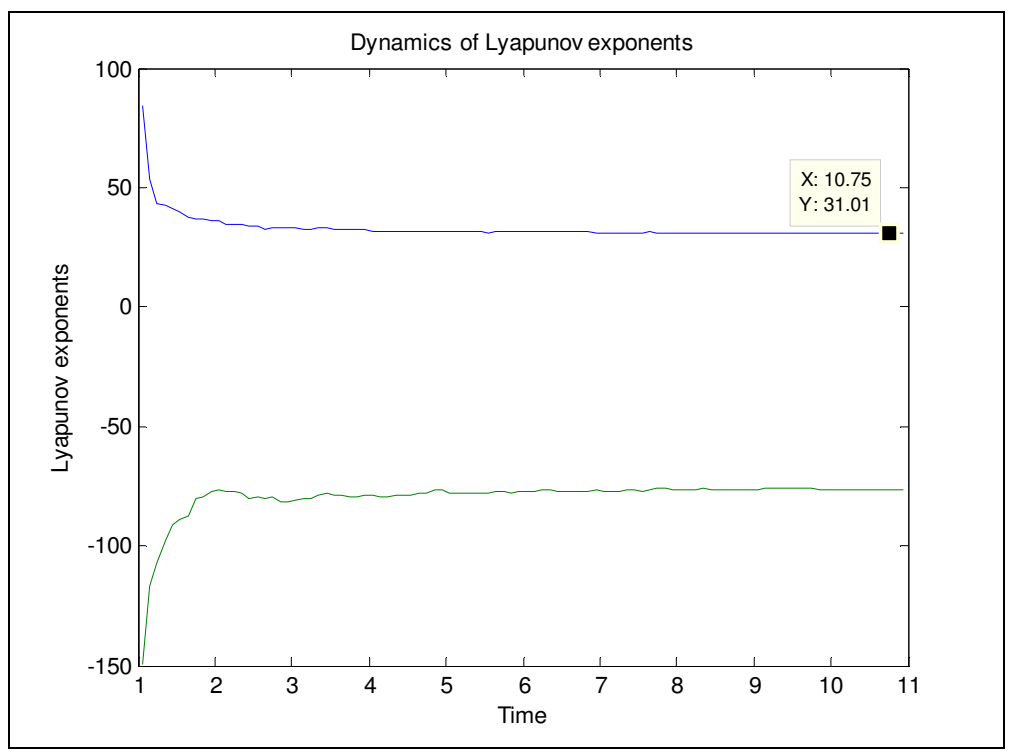

Fig. 64. Time Response of the Calculation of the Maximum (blue) and Minimum (green) Lyapunov Exponents at 14.5 Degrees of Misalignment Excited at $135 \mathrm{~Hz}$.

The system converges to a maximum Lyapunov exponent of 31.01. This confirms the presence of chaotic vibrations at this state of the system. A comparison of a known stable solution at 1 degree of misalignment is subsequently pictured in Fig. 65 . 


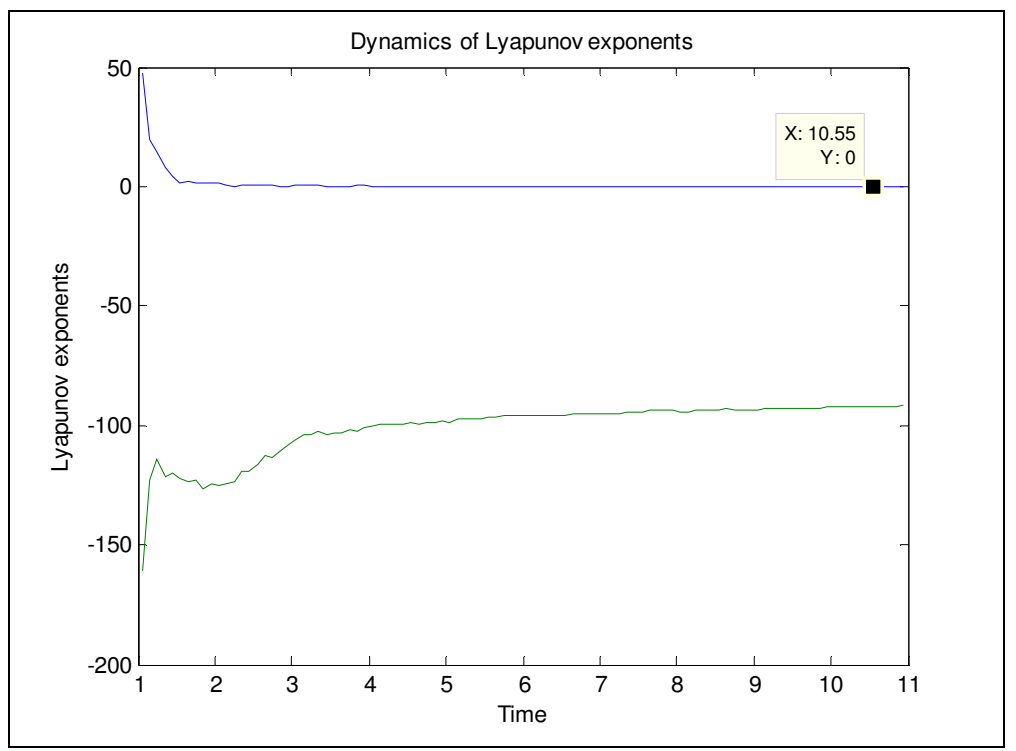

Fig. 65. Time Response of the Calculation of the Maximum (blue) and Minimum (green) Lyapunov Exponents at 1 Degree of Misalignment Excited at $135 \mathrm{~Hz}$.

The one degree of misalignment converges to a maximum Lyapunov exponent of zero. This confirms the expected result of nonchaotic vibrations. Therefore, running the analysis through a range of misalignment angles similar to that performed for the bifurcation and Poincare analysis would determine ranges of chaotic vibrations due to misalignment angles. A summary of the results is plotted in Fig. 66. 


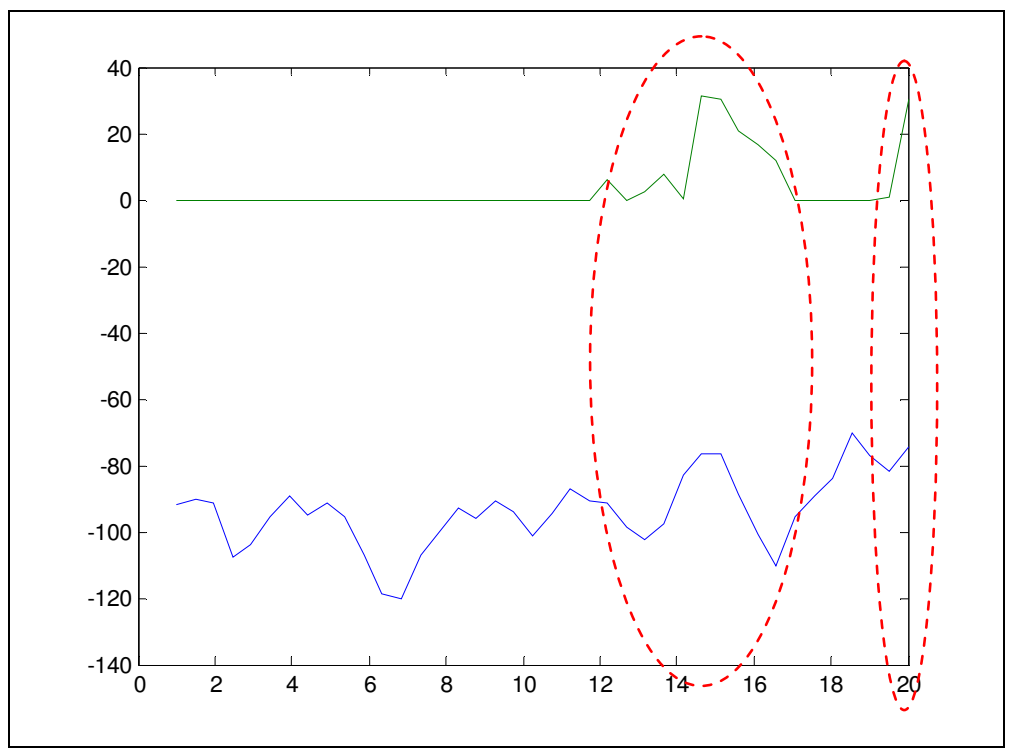

Fig. 66. Converged Maximum (green) and Minimum (blue) Lyapunov Exponents for Misalignment Angles of 1 to 20 Excited at $135 \mathrm{~Hz}$.

The maximum Lyapunov exponents confirm ranges of chaotic vibration. These ranges coincide with the ranges of multiple fequency solutions in the bifurcation, Poincare, and spectral analysises. These include the ranges of 11.8 to 17 degrees, and around 20 degrees of misalignment. An additional comparison, is that the value of the maximum Lyapunov exponent also quantifies the rate of divergence of the system. Therefore, the greater the value, the more chaotic the system. One again corresponding to the previous analysis, 14.5 degrees have a large maximum Lyapunov exponent, and it had the largest variation of first returns, and the most complex time waveform. For comparison, the time waveforms at various misalignement angles are presented in Fig. 67 to show the correlation of the waveforms with the Lyapunov exponent results. 


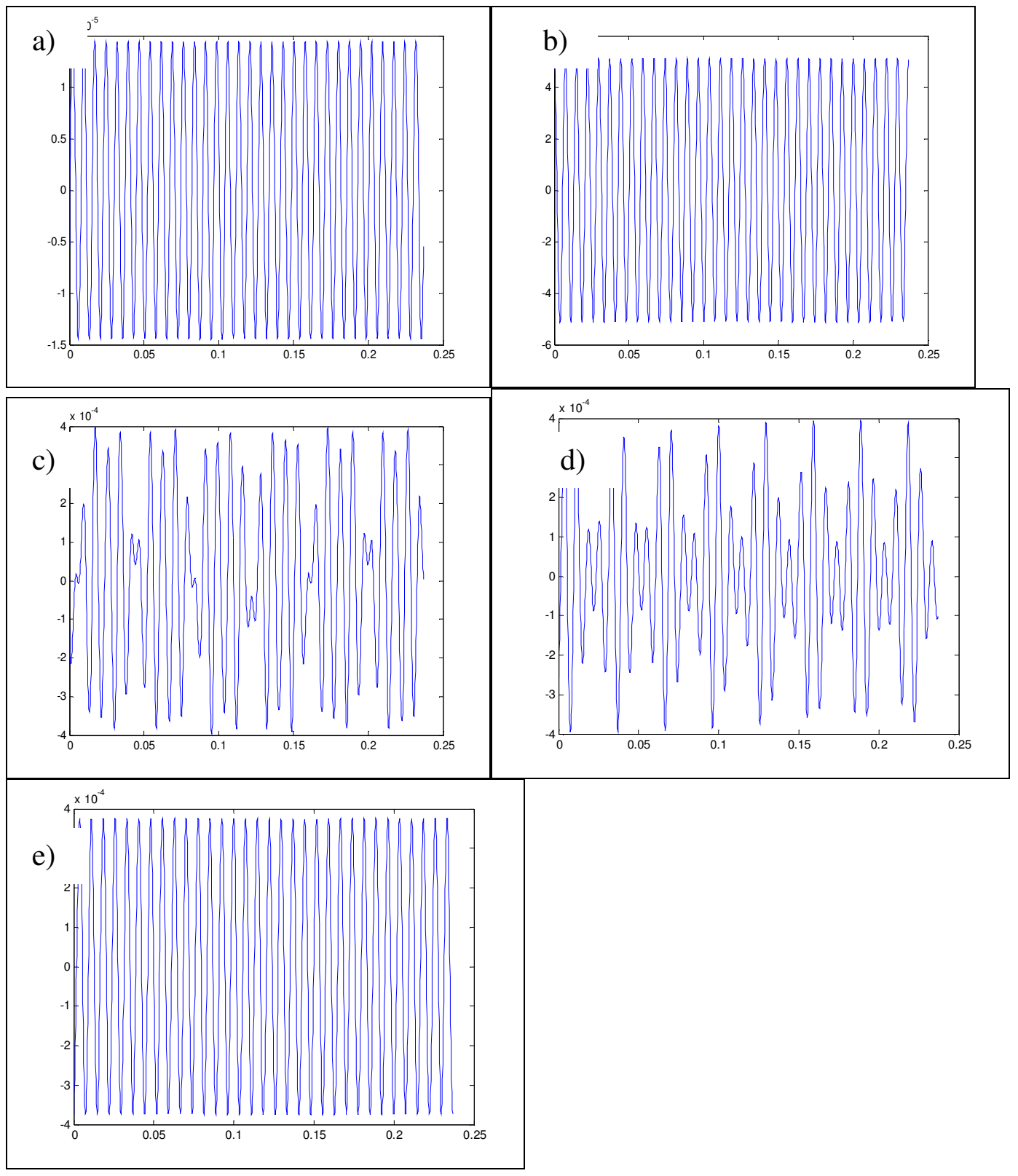

Fig. 67. Time Response of Front Driveshaft at ITD Connection Location for Misalignment Angles of: a) 1 , b) 10 , c) 14.3 , d) 16.7 , and e)18.5 Degrees.

The time waveforms for the various angles show a typical linear type response for 1, 10, and 18.5 degrees of misalignment. Conversely, the waveforms show complex 
multiple frequency response at misalignment angles of 14.3 and 16.7 degrees. This correlates with the expected results from the Lyapunov exponent results.

To determine the significance of the ITD nonlinear stiffness on the system's chaotic response, the Lyapunov Exponents were calculated for the same range of misalignment angles. The results of this calculation is presented in Fig. 68.

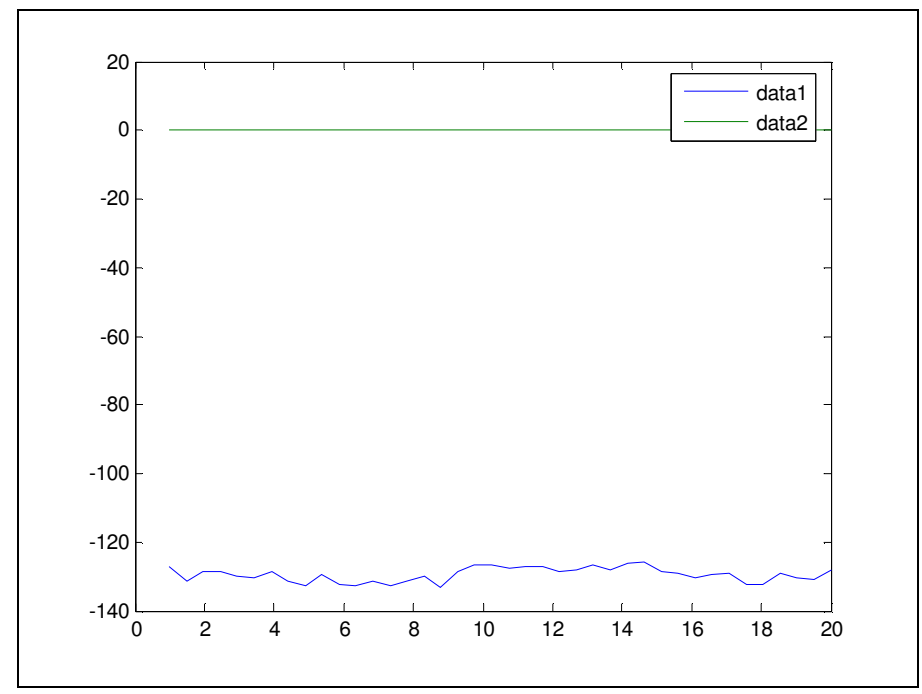

Fig. 68. Lyapunov Exponents for Misalignment Angles 0.5 - 20 Degrees without ITD Nonlinear Stiffness.

The Lyapunov Exponents are all equal to or less than zero. This indicates no evidence of chaotic vibrations. This is in stark contrast to ranges of misalignment angles producing chaotic vibrations with the ITD nonlinearity as previously presented in Fig. 66. Confirmation of the results was completed by completing the bifurcation analysis 
without the ITD nonlinear stiffness. The bifurcation diagram for this system is shown in Fig. 69.

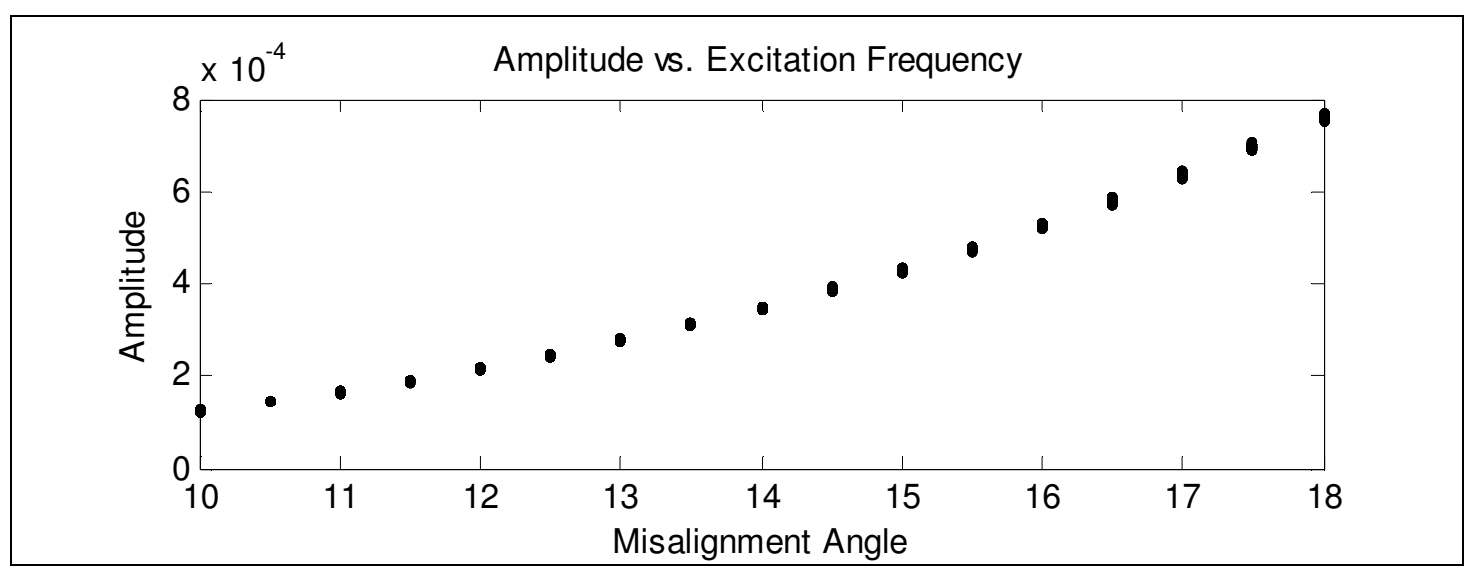

Fig. 69. Bifurcation of System without ITD Nonlinear Stiffness.

The bifurcation diagram shows only a coherent singular point of first returns.

Only a steady state coherent response to the $4^{\text {th }}$ order excitation is present without the ITD nonlinear stiffness. Therefore, the ITD nonlinear stiffness is required for the system to produce chaotic vibrations.

In summary, the driveshaft system exhibits chaotic vibration response at identified ranges of misalignment angles when excited by the $4^{\text {th }}$ order portion of the input force due to the universal joint with inclusion of the ITD nonlinear system. 


\section{Summary of Nonlinear System Analysis}

The derived model and force equation not only produced correlated results with a simulated run-up with test data, but also provided the framework for identifying unique nonlinear characteristics of the system. Bifurcation and Poincare analysis clearly identified the $4^{\text {th }}$ order excitation as the primary force influencing the jump in the experimental data. The analysis also showed possible additional jumps for the $2^{\text {nd }}$ order excitation as it would pass through the same resonance of the front driveshaft/ITD mode. The analysis showed ranges of misalignment angles at the front driveshaft/ITD resonant frequency of multiple frequency content. Spectral analysis confirmed both sideband type frequencies as well as sub-harmonic content. These ranges were narrowed down using Lyapunov exponents to be chaotic vibrations.

The analysis provided thus far shows interesting nonlinear response behavior. A significant characteristic of the nonlinear response of multiple solutions has not yet been identified. To identify multiple solutions, a brute force method was applied to generate multiple solutions for the same excitation frequency.

The run up and run down simulations were used to identify a set of initial conditions for finding multiple solutions. The simulation was stopped at the same excitation frequency and simulated with a constant excitation force from that point. The model was adjusted by reducing the damping to allow for a greater range of frequencies that have overlapped multiple solutions. Fig. 70 shows that the response from the run up and run down are not equivalent. 


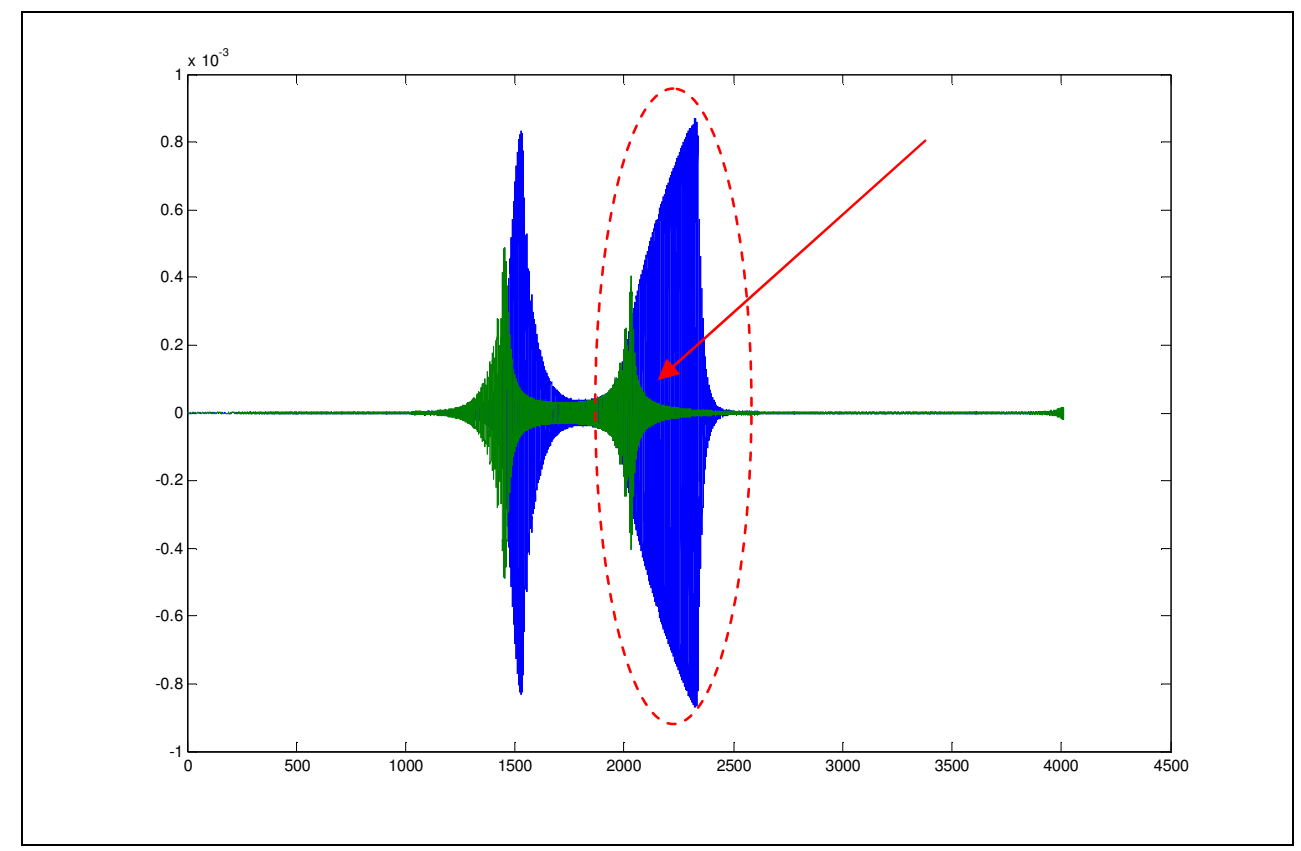

Fig. 70. Overlapping Plots of a Run Up (blue) and Run Down (green) Simulation with Reduced Damping.

The comparison of the run-up and run-down simulations show a distinct difference between the two scenarios as predicted by the nonlinear jump phenomena. The nonlinear jump has become more significant, and at a higher RPM/frequency. This provides easy access to a common frequency and shows how it should have multiple solutions. A frequency was chosen in the range of the different response as noted, and a steady state constant frequency excitation was applied and the system response was simulated. This simulation of 2 initial conditions to steady state provided the responses for one of the modal coordinates is plotted in Fig. 71. 


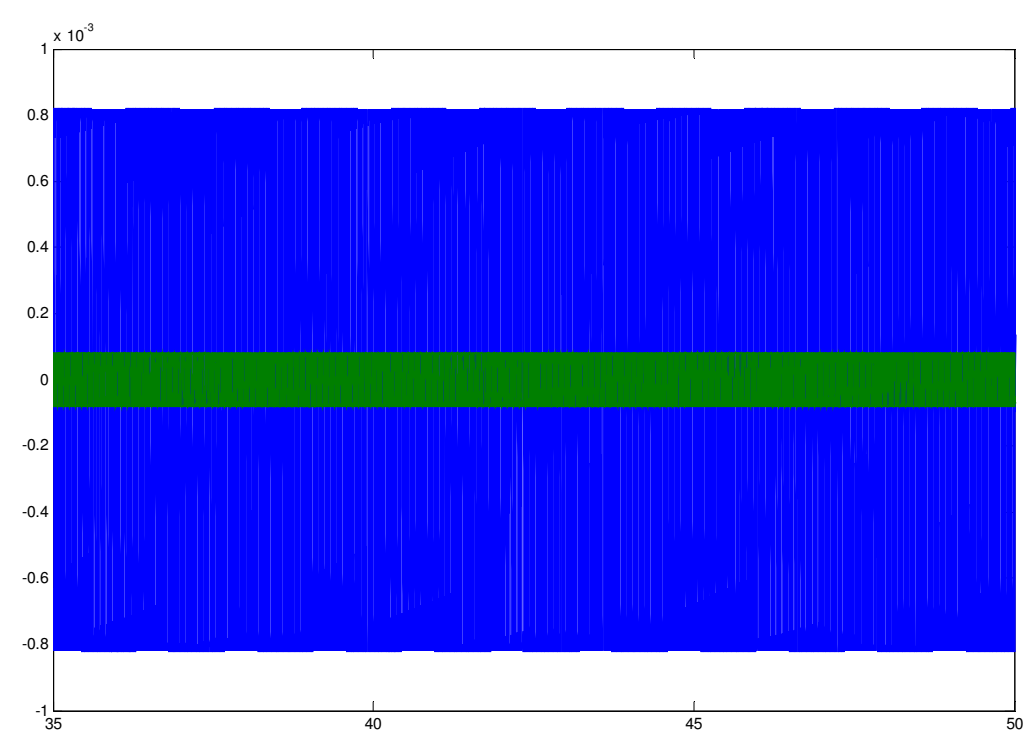

Fig. 71. Steady State $4^{\text {th }}$ Order Force Excitation Response Simulation from Run Up (blue) and Run Down (green) Initial Conditions

The larger amplitude is from the initial conditions upon run-up and the smaller from initial conditions from the run down at the same frequency. This correlates with the amplitudes obtained in the transient runs. A closer inspection of the time data provides evidence of the difference in response levels, but having the same frequency content. This can be noted in Fig. 72. 


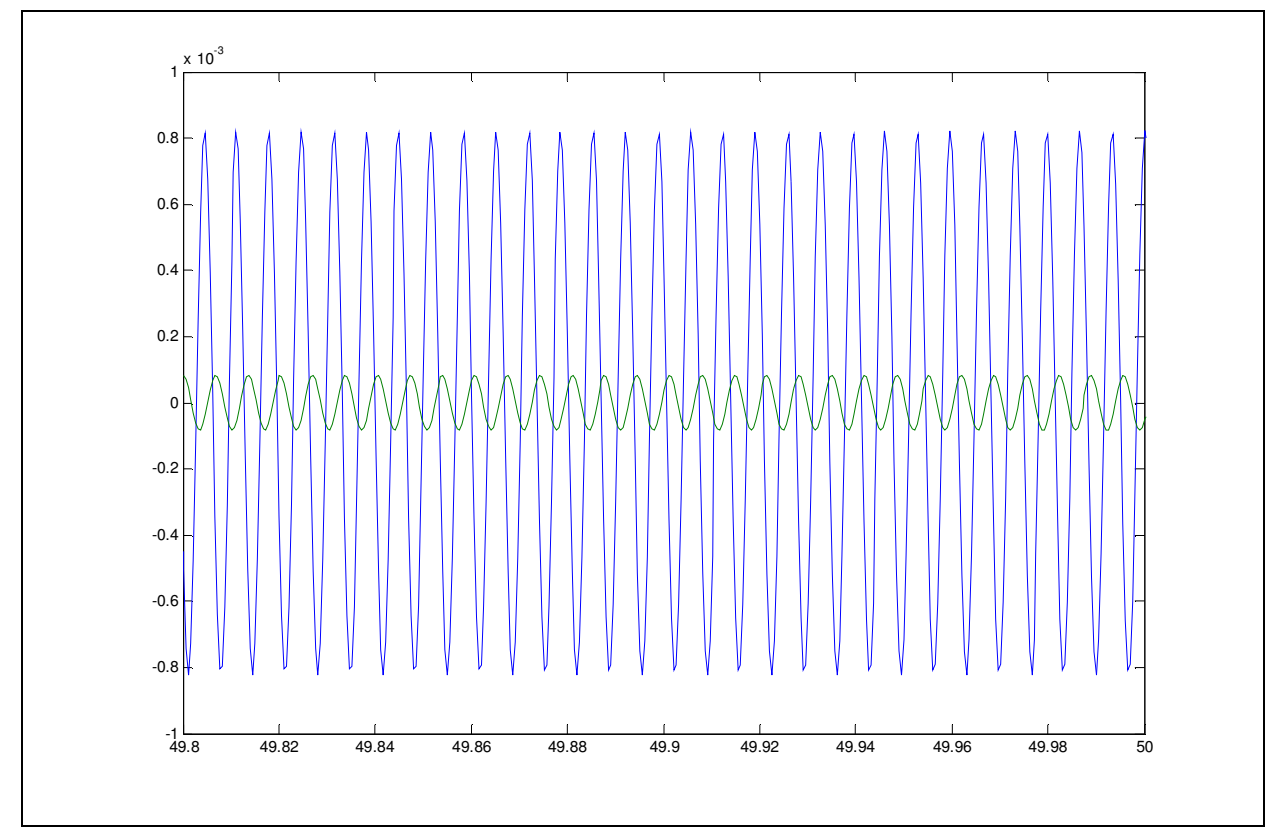

Fig. 72. Zoomed In on Time Period Steady State $4^{\text {th }}$ Order Force Excitation Response Simulation from Run Up (blue) and Run Down (green) Initial Conditions.

The responses of both simulations are at the same frequency, but with different amplitudes and phase response. This shows evidence of the expected multiple solutions in the nonlinear jump range. The nonlinear nature of the system and its various responses are confirmed. 


\section{CHAPTER VI}

\section{SUMMARY AND CONCLUSIONS}

Nonlinear vibration response of a common automotive driveshaft has been measured and explained by model characterization at the component and system levels, system simulation and correlation with the test data. The physical nonlinearity was isolated to the internal tuned damper which helps to reduce unwanted vibrations due to external forces, but not due to the universal joint related excitations.

The moment excitations were created by the misalignment angle producing a secondary moment as a resultant of the known geometric kinematics forcing the output shaft angle to vary from the input shaft angle. This secondary moment comprises of multiple even harmonics. The $4^{\text {th }}$ order harmonic is of special interest as it was isolated as the primary component of the input force that excited the ITD creating the jump.

Nonlinear analyses identified regions of special nonlinear response characteristics. Bifurcation and Poincare analyses identified regions with multiple frequency content. Spectral analysis confirmed the multiple frequencies, and attributed to some sub-harmonics and sidebands. Lyapunov analysis clearly identified these multiple frequencies as chaotic vibrations. Finally, multiple solutions for the same excitation due to different initial conditions, a key characteristic of nonlinear systems, were identified. 


\section{REFERENCES}

[1] I. Porat, Moment transmission by a universal joint, Mechanism and Machine Theory 15 (1980) 245-254.

[2] J. E. Baker, Displacement-closure equations of the unspecialized doubleHooke's-joint linkage, Mechanism and Machine Theory 37 (2002) 1127-1144.

[3] I. Hostens, J. Anthonis, H. Ramon, New design for a 6 dof vibration simulator with improved reliability and performance, Mechanical Systems and Signal Processing 19 (2005) 105-122.

[4] E. R. Wagner, C. E. Cooney, Section 3.1.1 Cardan of Hooke universal joint, The Society of Automotive Engineers: Universal Joint and Driveshaft Design Manual, Vol. 7, 1979, pp. 39-76.

[5] A. H. Berker, Appendix K: Basic mathematics for Cardan universal joint problems, The Society of Automotive Engineers: Universal Joint and Driveshaft Design Manual, Vol. 7, 1979, pp. 407-409.

[6] H. A. Desmidt, K. W. Wang, E. C. Smith, Coupled torsional-lateral stability of a shaft-disk system driven through a universal joint, Journal of Applied Mechanics 69 (2002) 261-273.

[7] B. Porter, A theoretical analysis of the torsional oscillation of a system incorporating a Hooke's joint, Journal of Mechanical Engineering Science 3 (1961) 324-329.

[8] B. Porter, Non-linear torsional oscillation of a two-degree-of-freedom system incorporating a Hooke joint, Proceedings of the Royal Society of London. Series A, Mathematical and Physical Sciences, Vol. 277, 1964, pp. 92-106.

[9] S. I. Chang, Torsional instabilities and non-linear oscillation of a system incorporating a Hooke's joint, Journal of Sound and Vibration 229 (2000) 9931002.

[10] S. F. Asokanthan, X.-H. Wang, Characterization of torsional instabilities in a Hooke's joint driven system via maximal Lyapunov exponents, Journal of Sound and Vibration 194 (1996) 83-91.

[11] S. F. Asokanthan, M.-C. Hwang, Torsional instabilities in a system incorporating a Hooke's joint, Journal of Vibration and Acoustics 118 (1996) 368-374. 
[12] S. F. Asokanthan, P. A. Meehan, Non-linear vibration of a torsional system driven by a Hooke's joint, Journal of Sound and Vibration 233 (2000) 297-310.

[13] A. J. Mazzei Jr., R. A. Scott, Principal parametric resonance zones of a rotating rigid shaft driven through a universal joint, Journal of Sound and Vibration 244 (2001) 555-562.

[14] A. J. Mazzei Jr., A. Argento, R. A. Scott, Dynamic stability of a rotating shaft driven through a universal joint, Journal of Sound and Vibration 222 (1999) 1947.

[15] M. F. Dimentberg, Transverse vibrations of rotating shafts: probability density and first-passage time of whirl radius, International Journal of Non-linear Mechanics 40 (2005) 1263-1267.

[16] M. F. Dimentberg, Random vibrations of a rotating shaft with non-linear damping, International Journal of Non-linear Mechanics 40 (2005) 711-713.

[17] J. Zahradka, Nonlinear oscillation in a two-degree-of-freedom mechanism caused by two Hooke's joints, Avtomaticheskaya Svarks, World Congress on Theory of Mach. and Mech. 1975, pp. 821-824.

[18] H. A. Desmidt, K. W. Wang, E. C. Smith, Stability of a segmented supercritical driveline with non-constant velocity couplings subjected to misalignment and torque, Journal of Sound and Vibration 277 (2004) 895-918.

[19] H. A. Desmidt, K. W. Wang, E. C. Smith, A. J. Provenza, On the robust stability of segmented driveshafts with active magnetic bearing control, Journal of Vibration and Control 11 (2005) 317-329.

[20] H. Ota, M. Kato, Lateral Vibrations of a rotating shaft driven by a universal joint (1st Report, Generation of even multiple vibrations by secondary moment), Bulletin of JSME 27(1984) 2002-2007.

[21] H. Ota, M. Kato, Even multiple vibrations of a rotating shaft due to secondary moment of a universal joint, Institution of Mechanical Engineers Conference Publications, 1984, pp. 199-204.

[22] M. Xu, R. D. Marangoni, Vibration analysis of a motor-flexible coupling-rotor system subject to misalignment and unbalance, part I: theoretical model and analysis, Journal of Sound and Vibration 176 (1994) 663-679. 
[23] R. H. Plaut, J. Wauer, Parametric, external and combination resonances in coupled flexural and torsional oscillations of an unbalanced rotating shaft, Journal of Sound and Vibration 183 (1995) 889-897.

[24] T. Iwatsubo, M. Saigo, Transverse vibration of a rotor system driven by a cardan joint, Journal of Sound and Vibration 95 (1984) 9-18.

[25] M. Kato, H. Ota, Lateral excitation of a rotating shaft driven by a universal joint with friction, Journal of Vibration and Acoustics 112 (1990) 298-303.

[26] R. M. Rosenberg, On the dynamical behavior of rotating shafts driven by universal (Hooke) coupling, Journal of Applied Mechanics 25 (1958) 47-51.

[27] P.-P. Sheu, W.-H. Chieng, A.-C. Lee, Modeling and analysis of the intermediate shaft between two universal joints, Journal of Vibration and Acoustics 118 (1996) 88-99.

[28] S. W. E. Earles, Some vibratory characteristics of Hooke's joint drive-line systems, Institution of Mechanical Engineers Conference Publications, 1984, pp. 63-70.

[29] M. Saigo, Y. Okada, Y. Ono, Self-excited vibration caused by internal friction in universal joints and its stabilizing method, Journal of Vibration and Acoustics 119 (1997) 221-229.

[30] P. B. Goncalves, Z. J. G. N. Del Prado, Low-dimensional Galerkin models for nonlinear vibration and instability analysis of cylindrical shells, Nonlinear Dynamics 41 (2005) 129-145.

[31] A. Jana, A. Raman, Nonlinear dynamics of a flexible spinning disk couples to a precompressed spring, Nonlinear Dynamics 40 (2005) 1-20.

[32] P. S. Beran, D. J. Liucia, A reduced order cyclic method for computation of limit cycles, Nonlinear Dynamics 39 (2005) 143-158.

[33] G. Kerschen, H.-C. Golinval, A. F. Vakakis, L. A. Bergman, The method of proper orthogonal decomposition for dynamical characterization and order reduction of mechanical systems: an overview, Nonlinear Dynamics 41 (2005) 147-169.

[34] W. Silva, Identification of nonlinear aeroelastic systems based on the Volterra Theory: progress and opportunities, Nonlinear Dynamics 39 (2005) 25-62. 
[35] C. H. Pak, On the coupling of non-linear normal modes, International Journal of Non-linear Mechanics 41 (2006) 716-725.

[36] N. Khader, Stability analysis for the dynamic design of rotors, Journal of Sound and Vibration 207 (1997) 287-299.

[37] C. Chang-Jian, Dynamics of dual rub-impact rotors/stators equipped with long journal bearings, Journal of the Chinese Society of Mechanical Engineers 29 (2008) 421-427.

[38] Y. Pu, J. Chen, J. Zou, The research on nonlinear characteristics of a cracked rotor and reconstruction of the crack forces, Preceedings of the Institution of Mechanical Engineers Part C - Journal of Mechanical Engineering Science Vol. 216, 2002, pp. 1099-1108.

[39] J. Xu, R. D. Marangoni, Vibration analysis of a motor-flexible coupling-rotor system subject to misalignment and unbalance, part II: experimental validation, Journal of Sound and Vibration 176 (1994) 681-691.

[40] J. C. Ji, Local bifurcation control of a forced single-degree-of-freedom nonlinear system: saddle-node bifurcation, Nonlinear Dynamics 25 (2001) 369-382.

[41] J. C. Ji, A. Y. T. Leung, Non-linear oscillations of a rotor-magnetic bearing system under superharmonic resonance conditions, International Journal of Nonlinear Mechanics 38 (2003) 829-835.

[42] W. Lacarbonara, F. Vestroni, Nonclassical responses of oscillators with hysteresis, Nonlinear Dynamics 32 (2003) 235-258.

[43] M. Browne, A. Palazzolo, Superharmonic nonlinear lateral vibrations of a segmented driveline incorporating a tuned damper excited by non-constant velocity joints Journal of Sound and Vibration (2009).

[44] K. Cuppens, P. Sas, L. Hermans, Evaluation of the FRF based substructuring and modal synthesis technique applied to vehicle FE data, IMSA 25 International Conference on Noise \& Vibration Engineering, 2000, pp. 1143-1150.

[45] D. W. Jordan and P. Smith, Nonlinear Ordinary Differential Equations, An Introduction to Dynamical Systems, Fourth Edition Oxford University Press, New York, 2007.

[46] A. Wolf, J. B. Swift, H. L. Swinney, J. A. Vastano, Determining Lyapunov exponents from a time series, Physica D: Nonlinear Phenomena, Vol. 16, 1985, pp. 285-317. 


\section{APPENDIX A \\ LYAPUNOV EXPONENT CALCULATION METHOD AND VERIFICATION}

Previously mentioned in the text, methods of calculation of the spectrum have been outlined in previous work $[45,46]$. These methods use an iterative approach to test for the value of the Lyapunov coefficients, and converge on a solution after many repetitions. A brief outline of the calculation routine will be presented for reference. In addition, trial systems with known results will be presented for both understanding the data analysis results, but for verifying the compilation code for calculating the Lyapunov exponents.

A set of $n$ nonlinear equations as well as n-sets of linearized equations are simulated through a time period. A sample set for the Duffing equation is

$$
\begin{aligned}
& \left\{\begin{array}{l}
\dot{q}_{1} \\
\dot{q}_{2} \\
\dot{q}_{3}
\end{array}\right\} \quad\left\{\begin{array}{c}
q_{2} \\
-K^{*} q_{2}+q_{1}-\left(q_{1}\right)^{3}+\Gamma\left(\cos \left(q_{3}\right)\right) \\
\omega
\end{array}\right\} \\
& \left\{\begin{array}{l}
\dot{q}_{l 1,1} \\
\dot{q}_{l 1,2} \\
\dot{q}_{l 1,3}
\end{array}\right\} \quad J\left(q_{1}, q_{2} q_{3}\right) *\left\{\begin{array}{l}
q_{l 1,1} \\
q_{l 1,2} \\
q_{l 1,3}
\end{array}\right\} \\
& \left\{\begin{array}{l}
\dot{q}_{l 2,1} \\
\dot{q}_{l 2,2} \\
\dot{q}_{l 2,3}
\end{array}\right\}=J\left(q_{1}, q_{2} q_{3}\right) *\left\{\begin{array}{l}
q_{l 2,1} \\
q_{l 2,2} \\
q_{l 2,3}
\end{array}\right\} \\
& \left\{\begin{array}{l}
\dot{q}_{l 3,1} \\
\dot{q}_{l 3,2} \\
\dot{q}_{l 3,3}
\end{array}\right\} \quad J\left(q_{1}, q_{2} q_{3}\right) *\left\{\begin{array}{l}
q_{l 3,1} \\
q_{l 3,2} \\
q_{l 3,3}
\end{array}\right\}
\end{aligned}
$$


where $J\left(q_{1}, q_{2} q_{3}\right)$ is the Jacobian of the Nonlinear equation evaluated at the current state of the nonlinear equation solution, and the $q_{l}$ are the linearized states. Initial condition vector would be defined by the initial conditions for evaluating the nonlinear equation, followed by a set of orthonormal unit length set of initial conditions for the linearized equations. For the Duffing equation, and example is

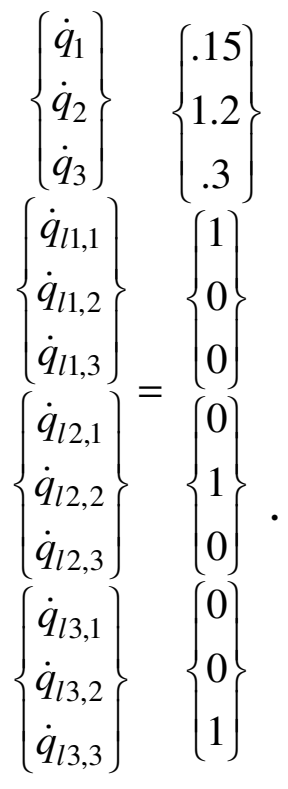

The full set of equations is integrated using any integration routine such as Runge-Kutta for the Lyapunov period defined. Multiple time steps for each period are necessary to integrate the equations, giving decent resolution of the linearized results. At the end of the simulation time period, a solution set of vectors will be generated. Since the vectors were originally orthonormal unit vectors, the simulated vector change would be simply the norm of the resultant vectors. 
Therefore, the final Lyapunov exponents are calculated as the solution of the time based average converges to a solution in

$$
\lambda_{i}=\frac{1}{t_{1}-t_{0}} \log _{n}\left|\left\{\begin{array}{l}
q_{l 1,1}\left(t_{1}\right) \\
q_{l 1,2}\left(t_{1}\right) \\
q_{l 1,3}\left(t_{1}\right)
\end{array}\right\}\right|,
$$

where $t_{1}$ and $t_{0}$ are the beginning and ending times for the Lyapunov period. The length of each vector identifies whether the process is converging or diverging.

For subsequent Lyapunov periods, the final sets of state vectors for the linearized equations are orthonormalized to unit vectors using a Gram-Schmidt procedure. After orthonormalizing to new sets of unit vectors, a new Lyapunov period is integrated. The subsequent approximation is the average of the change of lengths. Subsequent iterations are completed and the Lyapunov exponents at each iteration is calculated by

$$
\lambda_{i}=\frac{1}{t_{M}-t_{0}} \sum_{k=1}^{M} \log _{n}\left|\left\{\begin{array}{l}
q_{l 1,1}\left(t_{k}\right) \\
q_{l 1,2}\left(t_{k}\right) \\
q_{l 1,3}\left(t_{k}\right)
\end{array}\right\}\right|
$$

where $M$ is the total number of calculated Lyapunov exponent calculation periods assuming that an orthonormal unit length input vector.

The process iterates until the vectors and subsequently the Lyapunov exponents converge to a common length. It is proper to note that for each equation of motion, there will be a Lyapunov exponent. If the time factor is included as variable is in the Duffing 
example, $q_{3}$, there will always be a Lyapunov exponent that will be 0 , as the Jacobian terms will all be zero, and therefore the initial and final vectors will be the same.

The method was verified using documented known results from previous work $[45,46]$. This includes calculation and results for the Duffing, Lorenz, and RosslerChaos nonlinear systems. Each of these equations and subsequent results will be presented and produce the same results including the Lyapunov exponent values for the Maximum and minimum exponents respectively.

The Duffing equation was simulated and the Lyapunov exponents calculated for multiple levels of the system definition variables. The Duffing equation used is

$\ddot{x}+k \dot{x}-x-x^{3}=\Gamma \cos \omega t$

Where $k$ is the damping coefficient, $\Gamma$ is the amplitude of excitation, and $\omega$ is the input frequency. The following examples and comparisons are with a constant damping and input frequency, while various levels of the input amplitude are considered. May different responses are obtained, and the time domain and Lyapunov exponent analysis obtain consistent results.

Chaotic response is expected from the system with $\mathrm{k}=0.3, \omega=1.2$ and $\Gamma=0.5$. The Lyapunov Maximum and Minimum exponents and the simulated time response are presented in Fig. 73. 


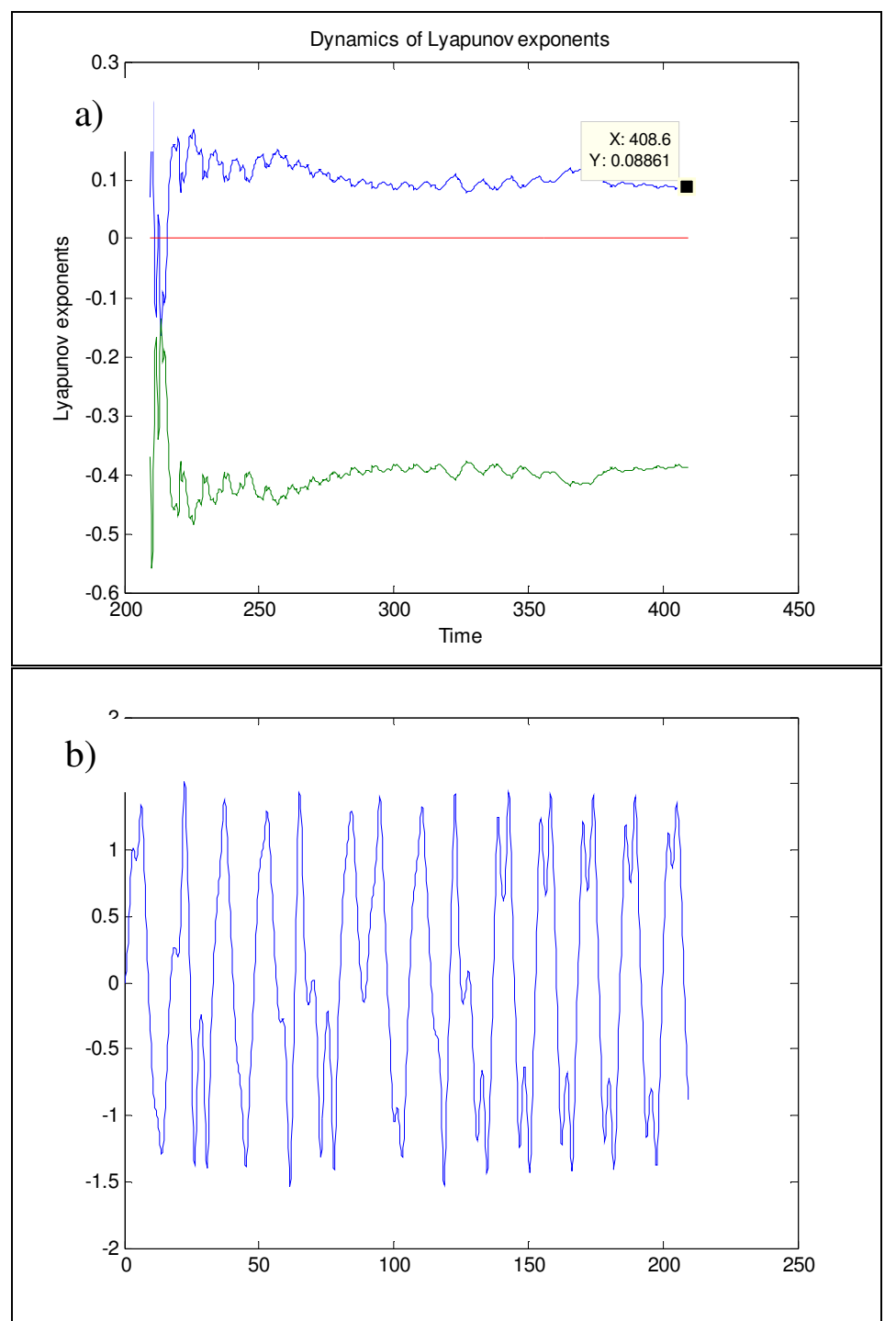

Fig. 73: Calculated Duffing Response with $k=0.3, \omega=1.2$, Gamma=0.5 of a) Lyapunov Maximum (blue) and Minimum (green) Exponents and b) Time Response.

The Lyapunov exponents predict chaotic vibrations with the Maximum Lyapunov Exponent being greater than 0 . The simulated time response also provides evidence of chaotic vibrations with significant variation of response over time. This can be compared to the response with a lower amplitude of excitation with $\Gamma=0.2$ in Fig. 74. 

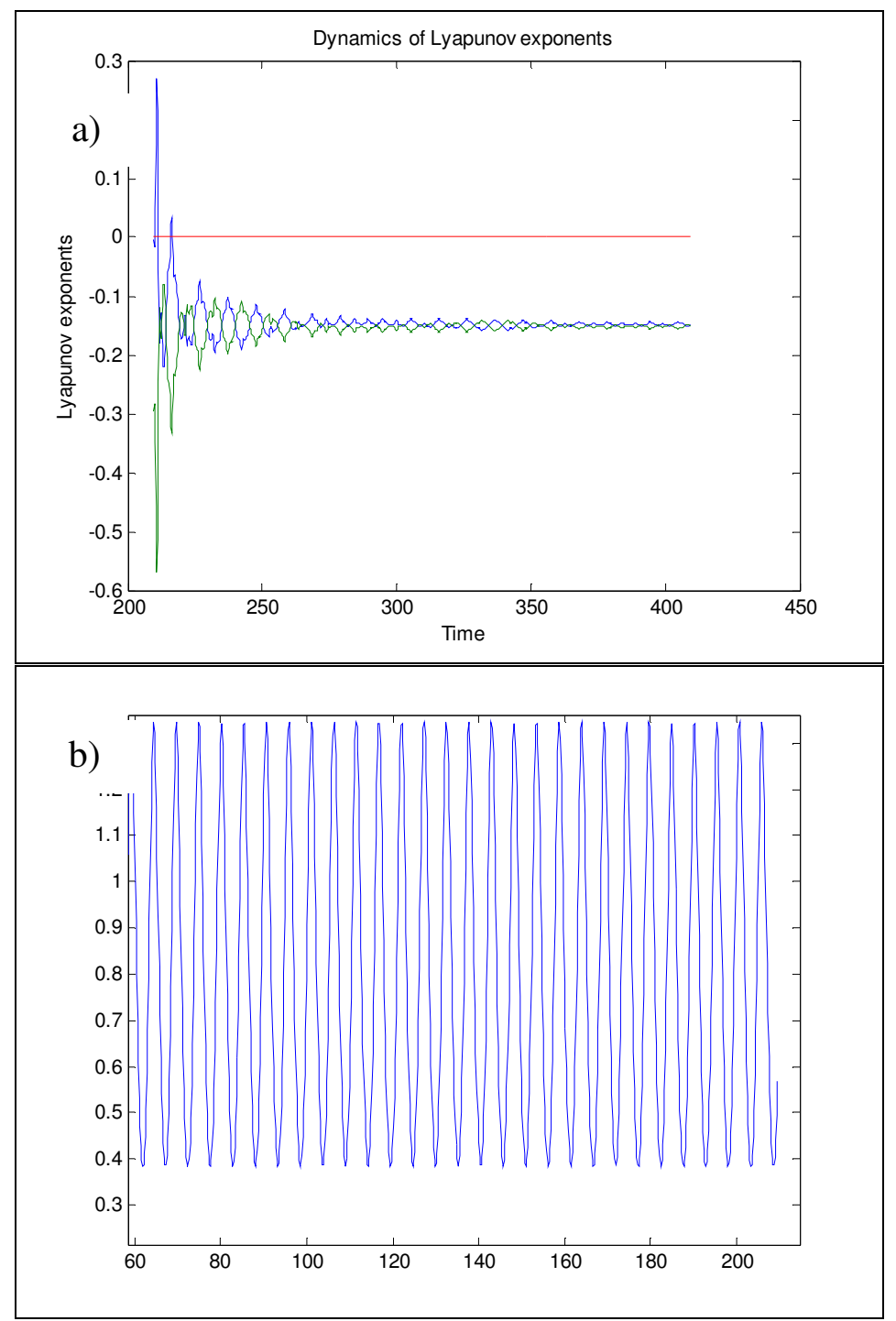

Fig. 74. Calculated Duffing Response with $\mathrm{k}=0.3, \omega=1.2$, Gamma=0.2 of a) Lyapunov Maximum (Blue) and Minimum (green) Exponents and b) Time Response.

The Lyapunov exponent analysis predicts a stable linear response as both the Maximum and Minimum exponents are less than zero. The time response provides a steady state harmonic response confirming the Lyapunov prediction. 
Intermediate levels of the input amplitude produce responses with harmonic and subharmonic components. The results with $\Gamma=0.28$ is plotted in Fig. 75 .
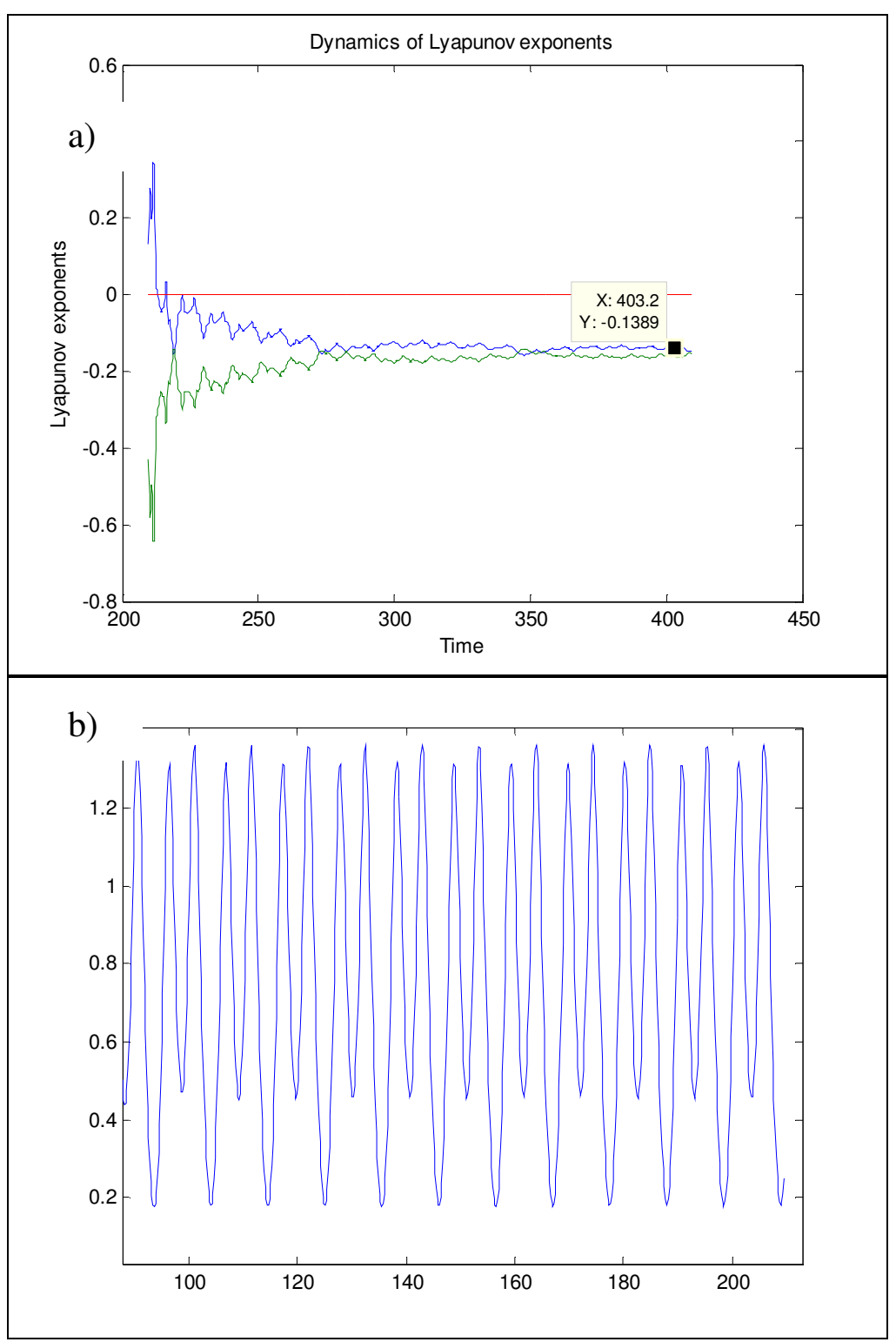

Fig. 75. Calculated Duffing Response with $k=0.3, \omega=1.2$, Gamma=0.28 of a) Lyapunov Maximum (blue) and Minimum (green) Exponents and b) Time Response. 
The Lyapunov exponents predict stable response characteristics as the Maximum Lyapunov exponent is less than zero. The time simulation results show a stable response with both a harmonic and a period 2 subharmonic. Therefore, the stable subharmonic response was predicted by the level of the Lyapunov exponents. A secondary case with additional subharmonic content is presented in Fig. 76 with excitation amplitude of $\Gamma=0.29$.

Once again, the Lyapunov analysis predicts stable response characteristics. The time response shows a harmonic, period 2 and period 4 subharmonics. Comparing the results, the Maximum Lyapunov exponent is less negative, showing the relatively less stable condition with the additional subharmonic content. This pattern is further noted with $\Gamma=0.37$ in Fig. 77.

The Lyapunov exponent still predicts a stable response. The time domain presents a stable periodic response, but with more complexity than previously presented. As the excitation level nears the predicted chaotic level of $\Gamma=0.5$ as presented in Fig. 73, the maximum Lyapunov exponent increases. A similar trend can be noted with impute amplitude levels exceeding the identified chaotic level as shown in Fig. 78. 

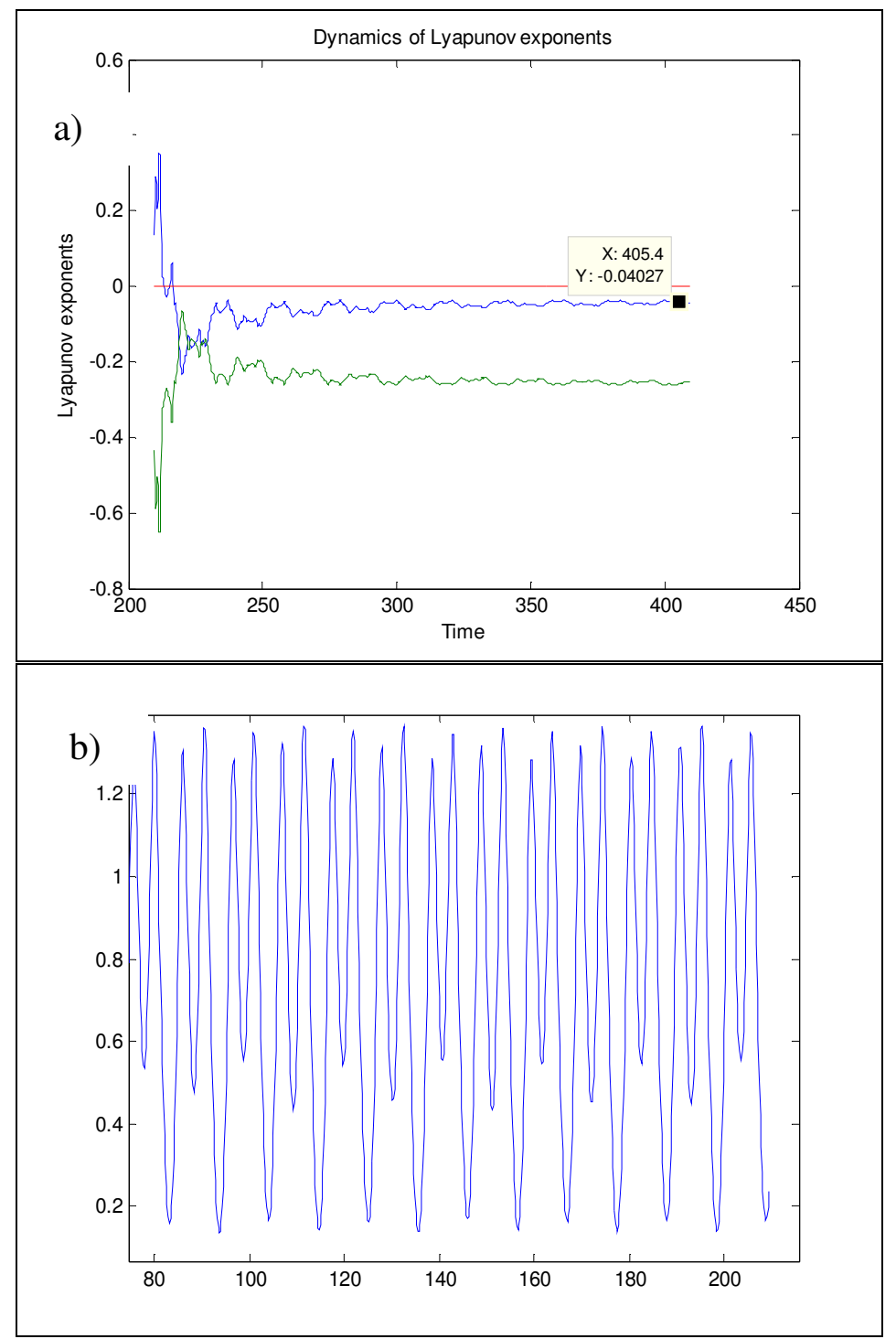

Fig. 76. Calculated Duffing Response with $\mathrm{k}=0.3, \omega=1.2$, Gamma=0.29 of a) Lyapunov Maximum (blue) and Minimum (green) Exponents and b) Time Response. 

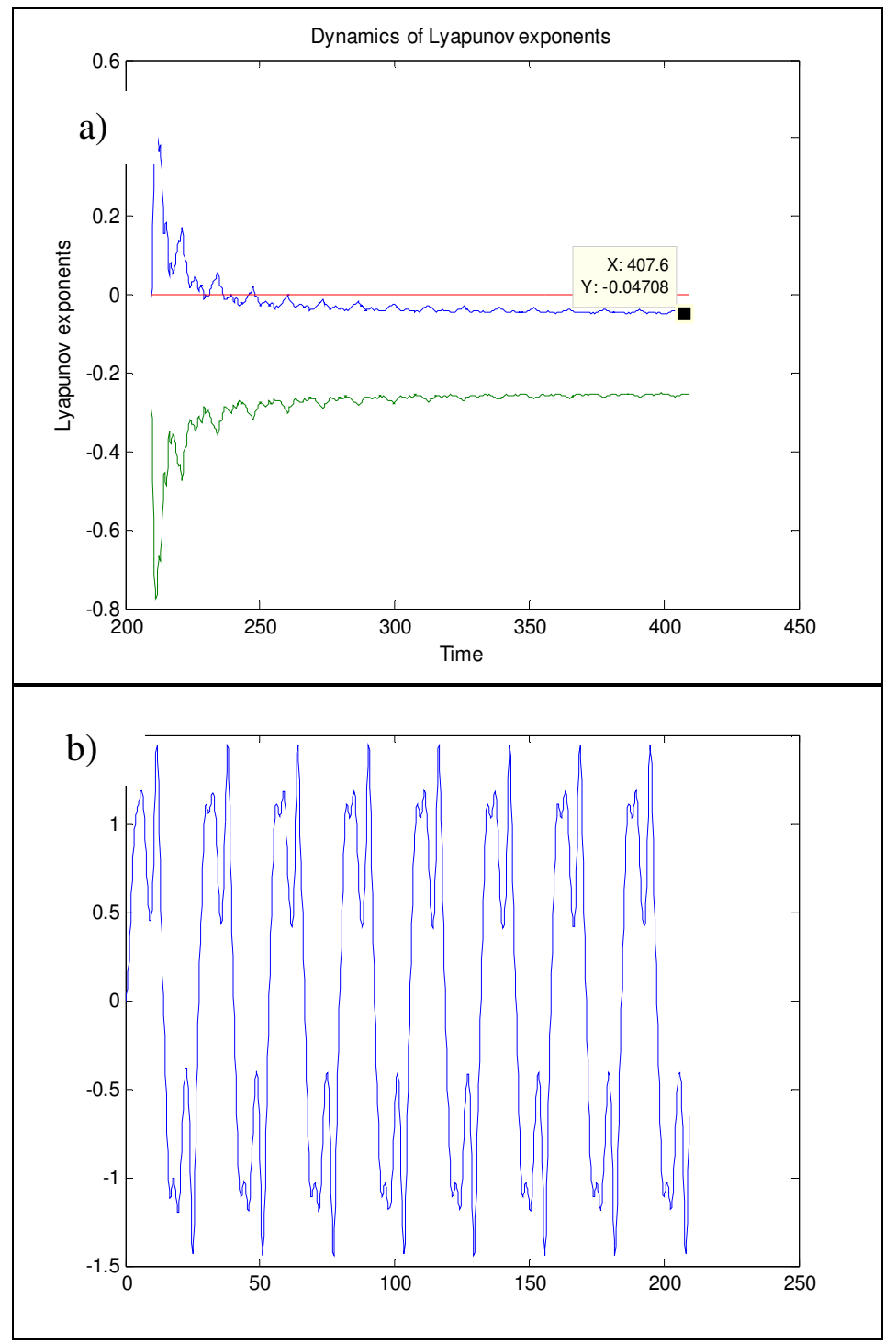

Fig. 77. Calculated Duffing Response with $\mathrm{k}=0.3, \omega=1.2$, Gamma=0.37 of a) Lyapunov Maximum (blue) and Minimum (green) Exponents and b) Time Response. 

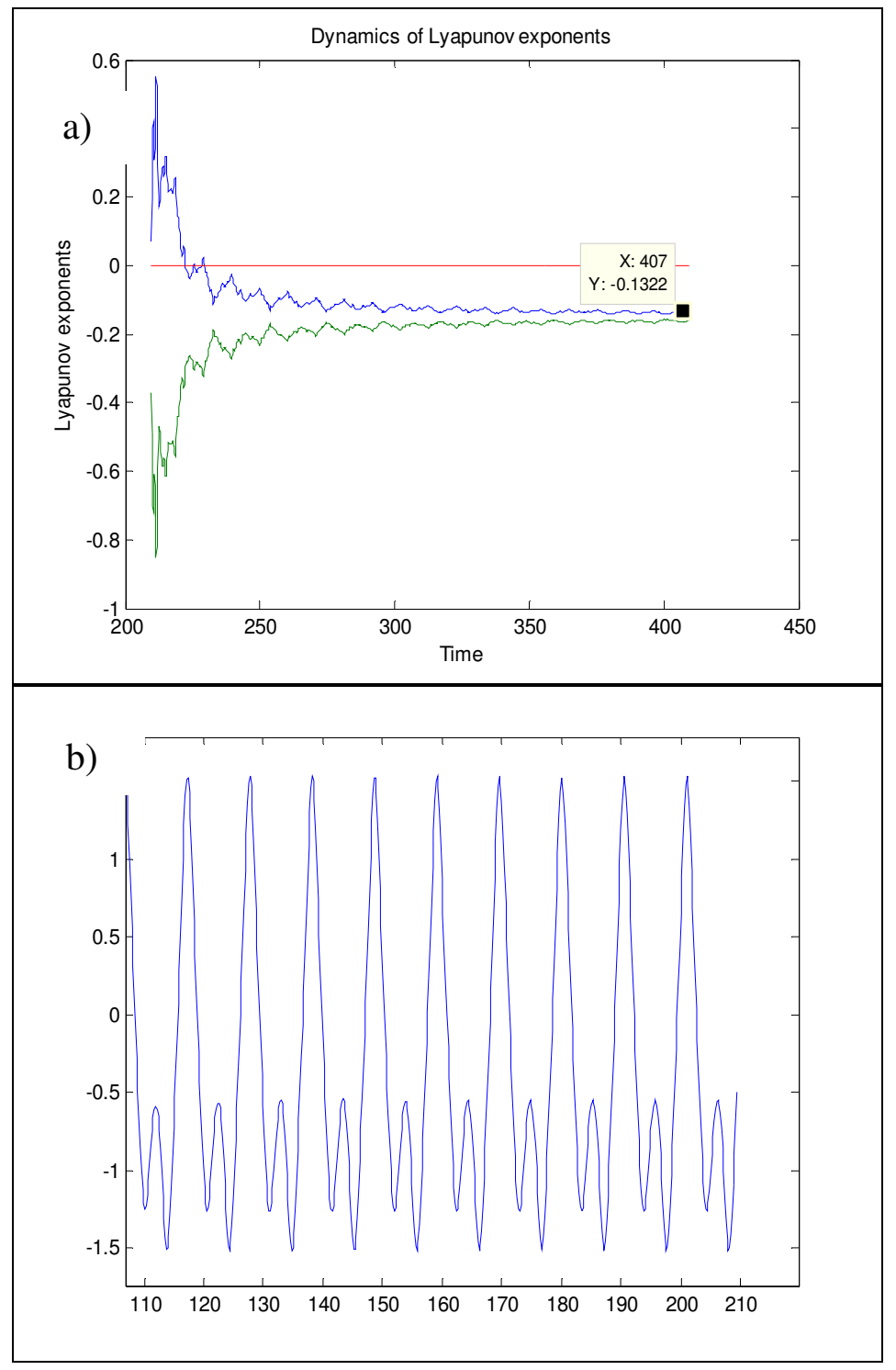

Fig. 78. Calculated Duffing Response with $\mathrm{k}=0.3, \omega=1.2$, Gamma=0.65 of a) Lyapunov Maximum (blue) and Minimum (green) Exponents and b) Time Response.

The Lyapunov exponents predict a stable response with $\Gamma=0.65$. The time response shows harmonic and a period 2 subharmonic content. Therefore, confirming the results. Furthering the noted trend, $\Gamma=0.73$ is plotted in Fig. 79. 

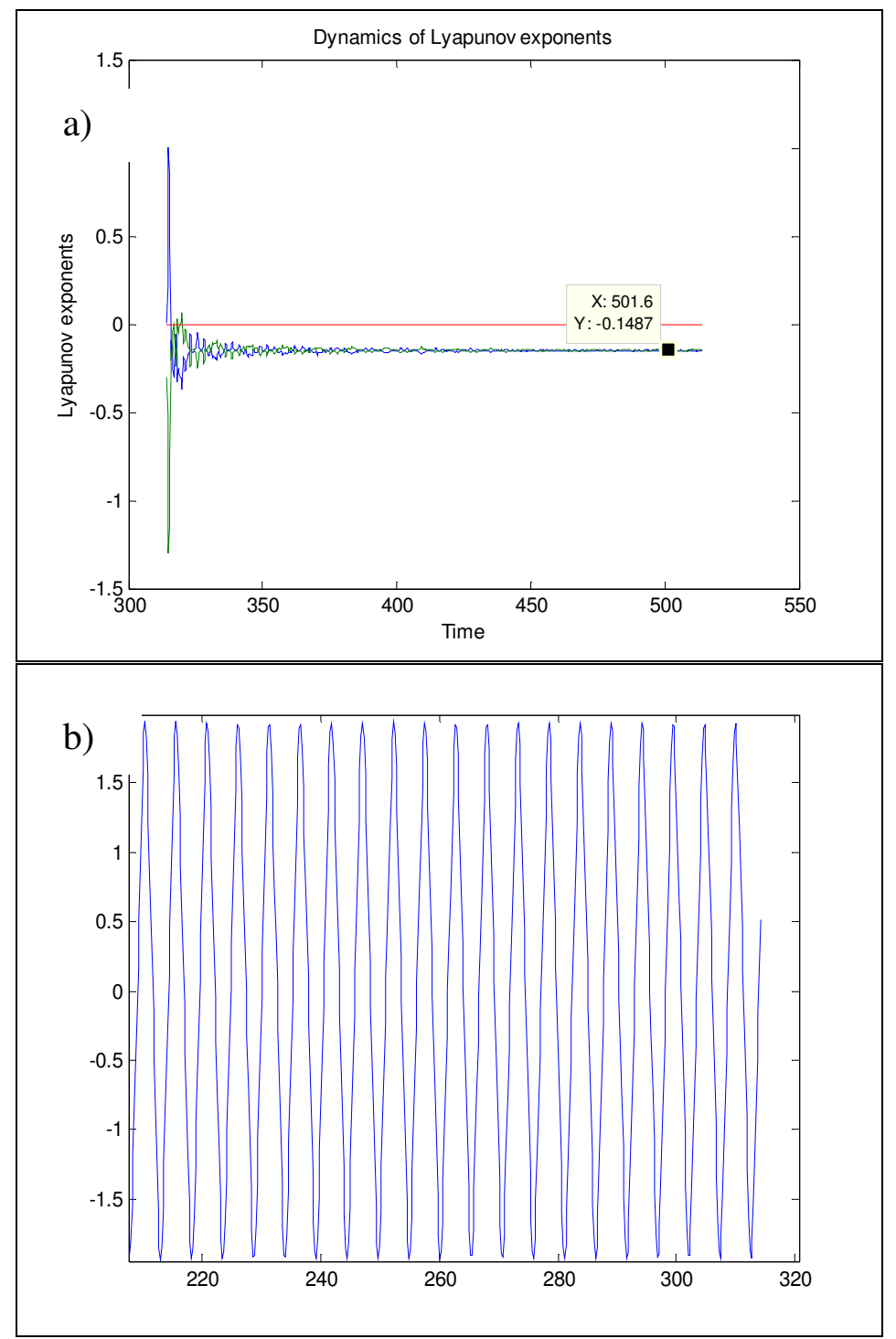

Fig. 79. Calculated Duffing Response with $k=0.3, \omega=1.2$, Gamma=0.73 Pre-simulation Start of 1.5,0 of a) Lyapunov Maximum (blue) and Minimum (green) Exponents and b) Time Response.

The maximum Lyapunov exponent is lower than with $\Gamma=0.65$, and shows a stable harmonic response. Therefore, the Lyapunov exponent analysis has correctly identified the chaotic and the relative complexity of the stable responses. At this level of input amplitude starting the simulation with different initial conditions reveals a different response. The Lyapunov exponent and time response are pictured in Fig. 80. 
Once again, the Lyapunov exponent analysis correctly predicts a chaotic response, verified by the tie response at this condition. This nonlinear response with multiple solutions at this state can have both stable and chaotic vibration responses.

The Lyapunov analysis can quantify other systems and identify the chaotic characteristics of systems. Another system, Lorenz, consists of three first order differential equations. This system is defined by

$$
\begin{aligned}
& \dot{X}=\sigma(Y-X) \\
& \dot{Y}=X(R-Z)-Y \\
& \dot{Z}=X Y-b Z
\end{aligned}
$$

where $\sigma, R$, and $b$ are parameters that can be defined. One setting with the parameters of $\sigma=16, \mathrm{R}-45.92$, and $\mathrm{b}=4$ using the natural $\log$ as the basis produced the results presented in Fig. 81. 

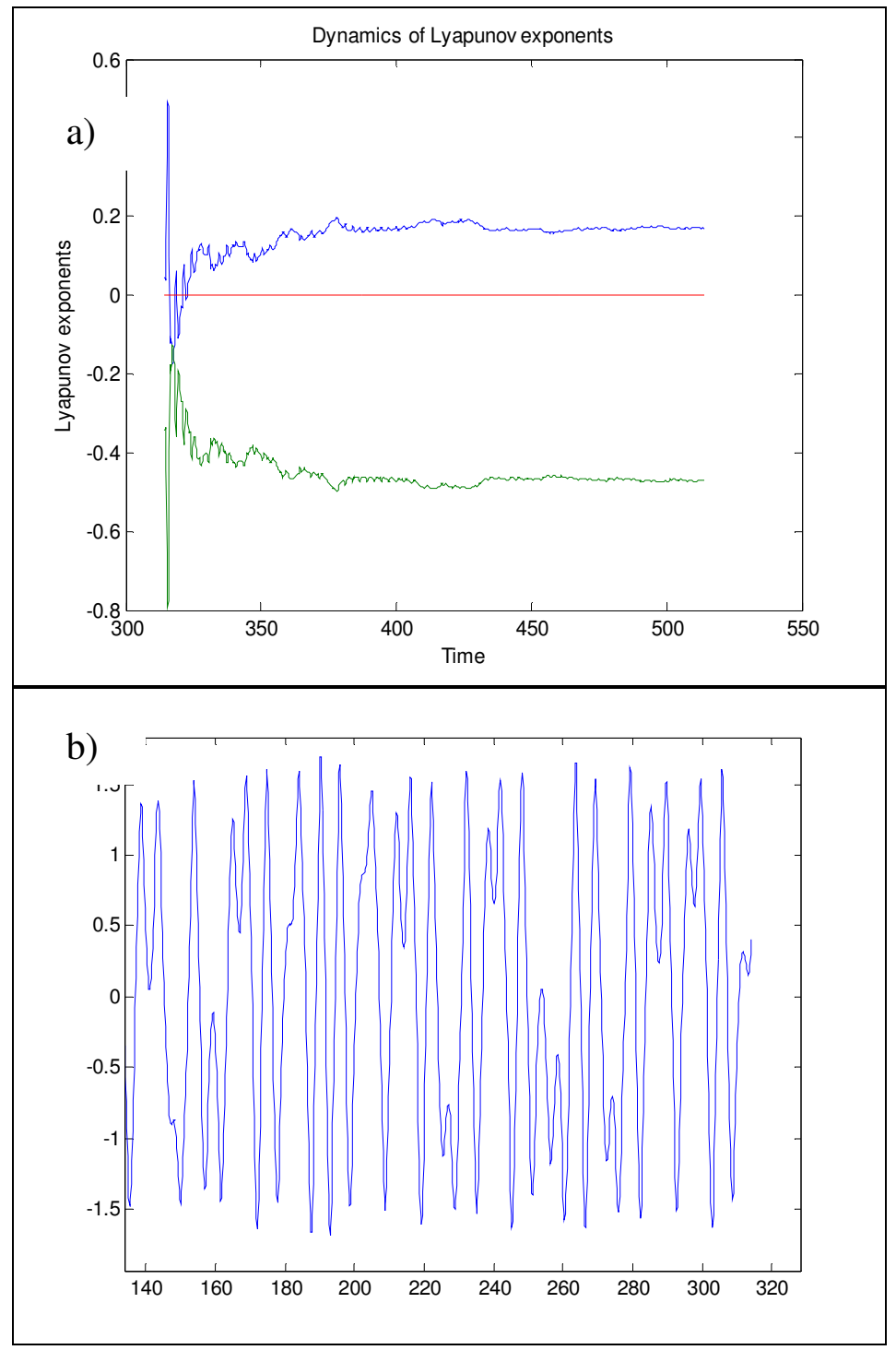

Fig. 80. Calculated Duffing Response with $k=0.3, \omega=1.2$, Gamma=0.73 Pre-simulation Start of 0,0 of a) Lyapunov Maximum (blue) and Minimum (green) Exponents and b) Time Response. 


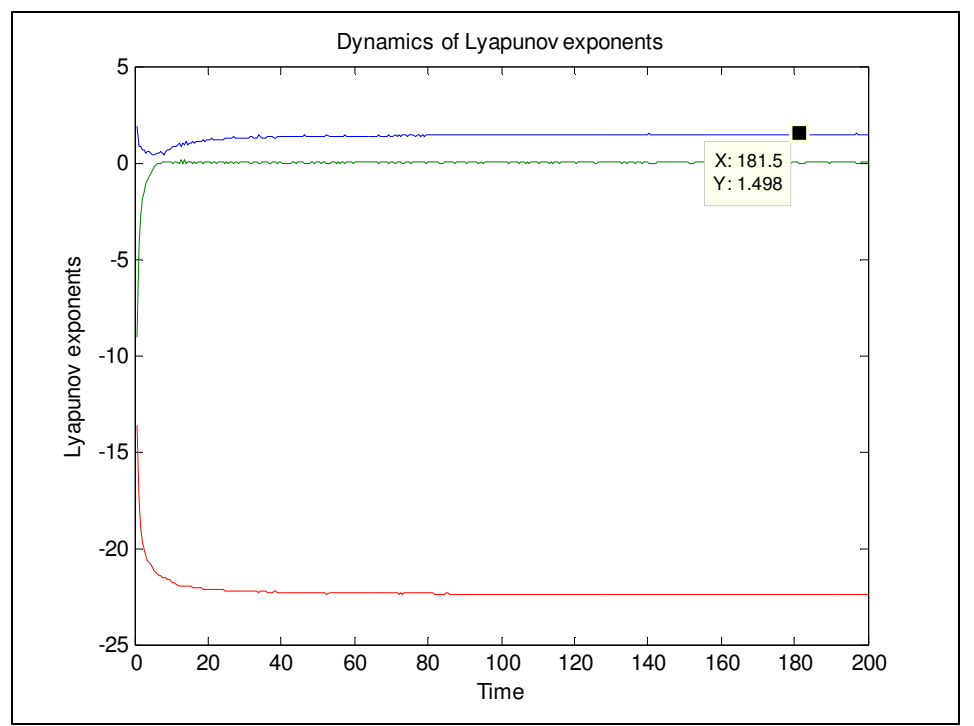

Fig. 81. Lyapunov Maximum (blue) and Minimum (red) Exponents for the Lorenz Equation with $\sigma=16, R=45.92$, and $b=4$ Using Natural $\log$.

The results show the same interpretation as Wolf with a positive, zero, and negative Lyapunov exponents. The amplitude differs from that reported by Wolf et al. Upon further inspection, Wolf et al. used log base 2 for the calculation, and therefore the amplitude would be different, but the signs would not be affected. Recalculating the Lyapunov exponents with Log base 2 used by Wolf et al produced the results shown in Fig. 82. 


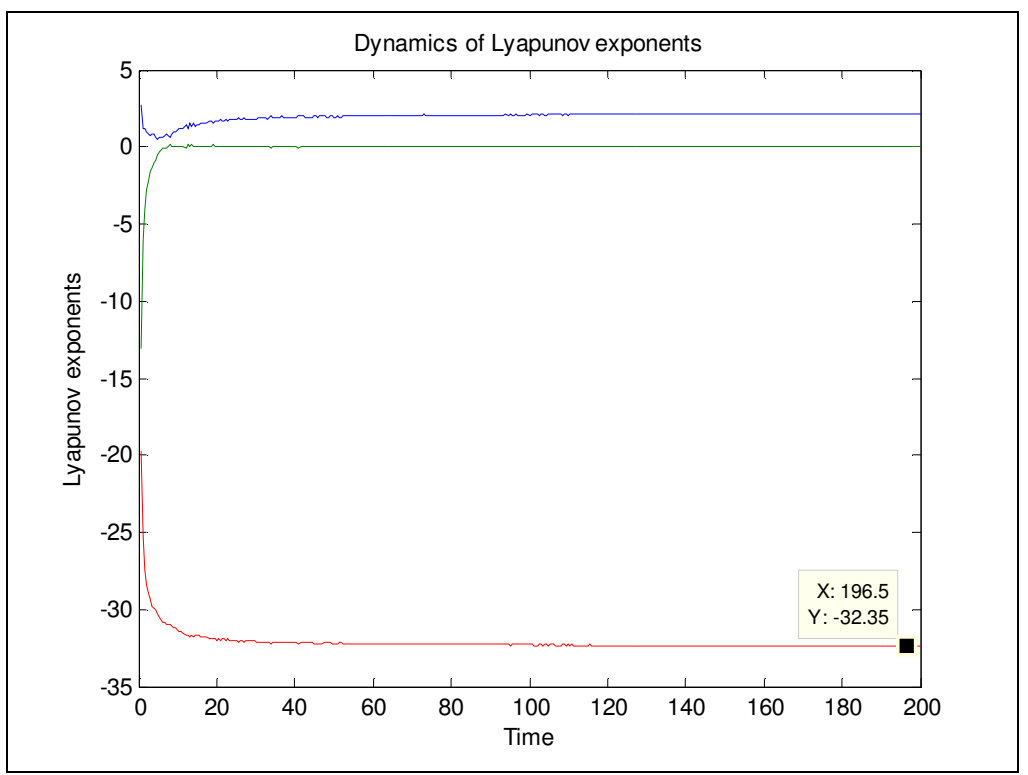

Fig. 82. Maximum (blue) and Minimum (red) Exponents for the Lorenz Equation with Sigma=16, $T=45.92$, and Beta=4 Using Log Base 2.

The results consisting of Lyapunov exponents of 2.16, 0, and -32.4 now match those presented by Wolf et al. Once again the sign is important and the relative amplitudes using the same basis for logs will indicate the complexity of the response.

A final system, Rossler Chaos, was evaluated to verity the calculation. This system also has three first order differential equations. The system is defined by:

$$
\begin{aligned}
& \dot{X}=-(Y+Z) \\
& \dot{Y}=X+a Y \\
& \dot{Z}=b+Z(X-c)
\end{aligned}
$$

where $\mathrm{a}, \mathrm{b}$, and $\mathrm{c}$ are parameters that are defined. The Lyapunov exponents are plotted in Fig. 83. 


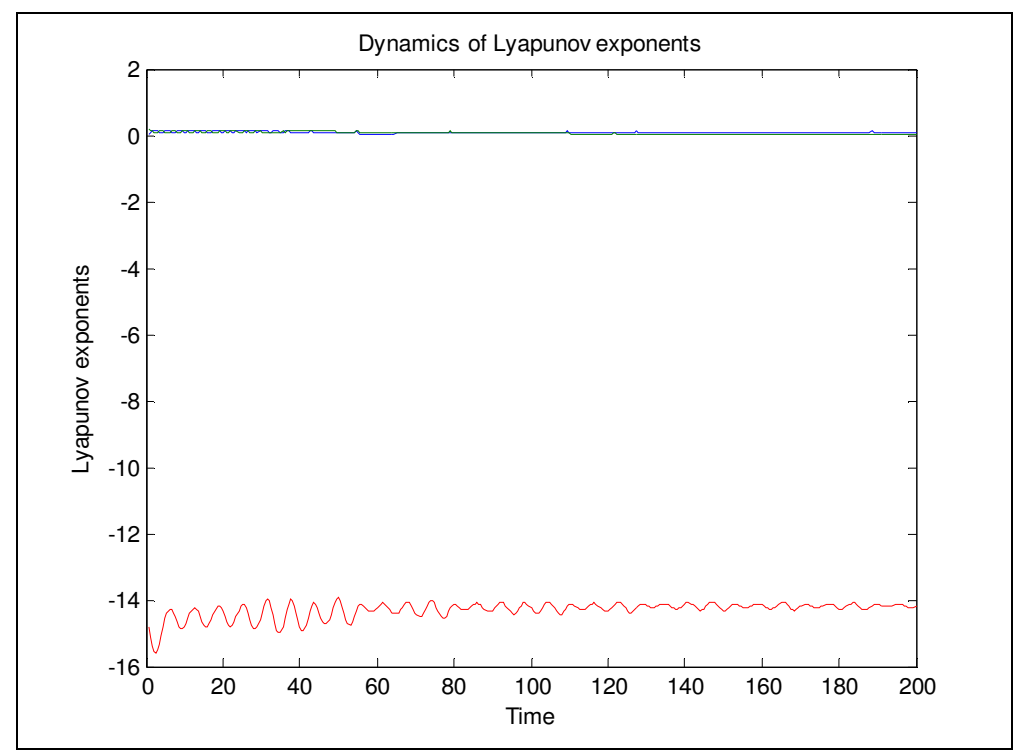

Fig. 83. Maximum (blue) and Minimum (red) Exponents for the Rossler Chaos with $a=0.15, b=.20$, and $c=10.0$ Using Log Base 2.

Rossler Chaos system produces Lyapunov exponents with the parameters of $\mathrm{a}=0.15, \mathrm{~b}=0.20$, and $\mathrm{c}=10.0$ indicating chaos. This is with exponents of $0.095,0.029$, and -14.15 agreeing closely with Wolf et al. obtaining results of $0.13,0.00$, and -14.1.

The Lyapunov calculation method is an efficient method of clearly identifying chaotic response. The method has agreed interpretation and levels with previously presented work with simpler equations. The amplitude of the Lyapunov exponents can help quantify not only whether a system is chaotic, but to the degree of complexity of the systems response. 


\section{VITA}

Michael Scott Browne received his Bachelor of Science degree in Mechanical Engineering from Brigham Young University in 1999. Initially working for Ford Motor Company, he transferred to Visteon Corporation and completed his Master of Science in Mechanical Engineering from The University of Michigan in 2001. Mr. Browne completed a Ph. D. from Texas A\&M University in May of 2009. Mr. Browne currently has 9 years of automotive engineering experience specializing in driveline and powertrain vibrations. His research interests include automotive noise and vibration testing and analysis, gear quality testing and analysis, systems integration, and AWD system controls.

Mr. Browne may be reached at McLaren Performance Technologies, Travelers Tower 1, 26555 Evergreen Road, Suite 900, Southfield, MI 48076. His email is: mike.browne@linamar.com. 